\title{
Functional analysis of Prdm14 during Xenopus embryogenesis
}

\author{
Doctoral Thesis
}

\author{
Dissertation for the award of the degree \\ "Doctor rerum naturalium (Dr. rer. nat.)" \\ in the GGNB program "Genes and Development" \\ at the Georg August University Göttingen \\ Faculty of Biology
}

Submitted by

Rolf Patrick Berndt

born in Tokio, Japan 


\section{Members of the Thesis Committee:}

Dr. Kristine A. Henningfeld (Supervisor, Reviewer)

Institute of Developmental Biochemistry,

University of Göttingen

Prof. Dr. Gregor Bucher (Reviewer)

Department of Evolutionary Developmental Genetics, University of Göttingen

Prof. Dr. Andreas Wodarz

Department of Microscopic Anatomy and Molecular Cell Biology, University of Cologne 


\section{Affidavit}

Herewith, I declare that I prepared the PhD thesis

"Functional analysis of Prdm14 during Xenopus embryogenesis"

on my own and with no other sources and aids than quoted.

Submission date

Göttingen, 30.04.2015

Rolf Patrick Berndt 


\section{Table of Contents}

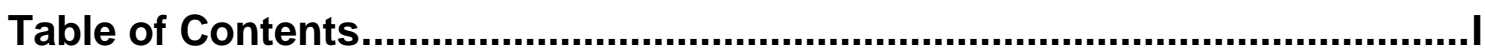

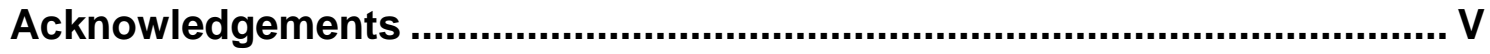

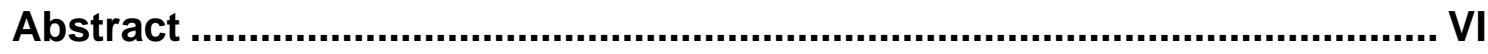

List of Figures ................................................................................ VIII

List of Tables.............................................................................................. XI

Abbreviations ............................................................................................. XII

1. Introduction ....................................................................................... 1

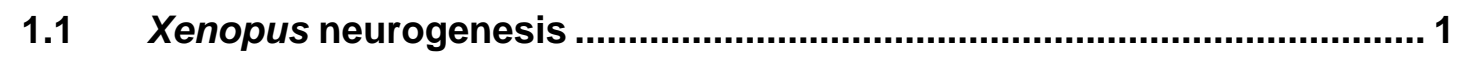

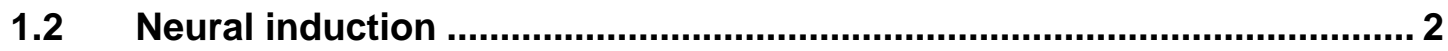

1.3 Stabilization of the neural fate............................................................ 5

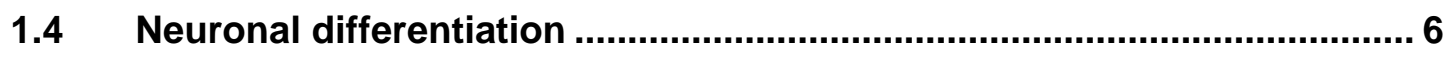

1.5 Neurulation and neuronal subtype specification ................................. 7

1.6 Lateral inhibition............................................................................... 9

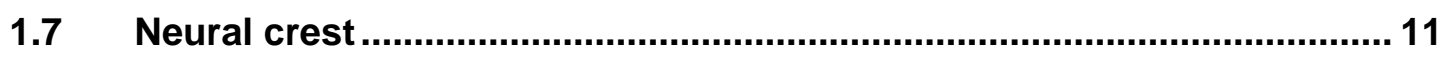

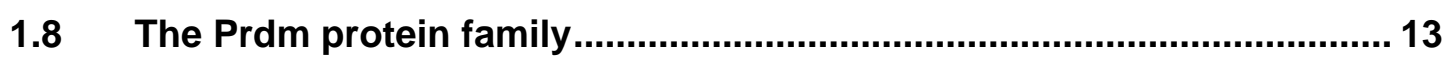

1.8.1 Structural properties of Prdm proteins ................................................ 13

1.8.2 The role of Prdms in early vertebrate neurogenesis and neural crest

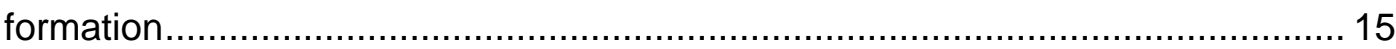

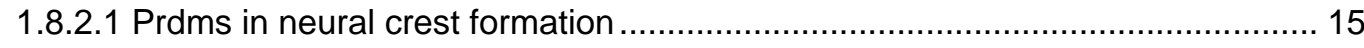

1.8.2.2 Regulation of neuronal subtype specification by Prdms ............................. 16

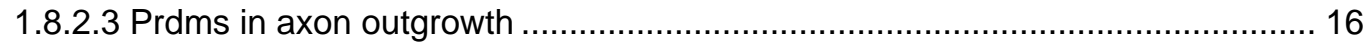

1.8.3 Prdm14 is an epigenetic regulator and stemness factor .................... 17

1.9 Aims .......................................................................................... 18

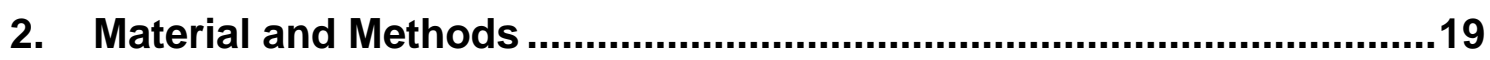

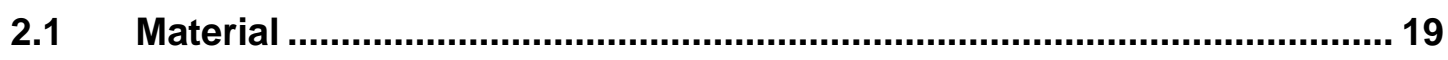

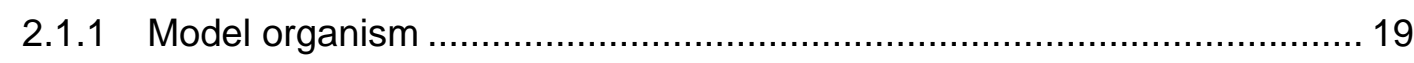

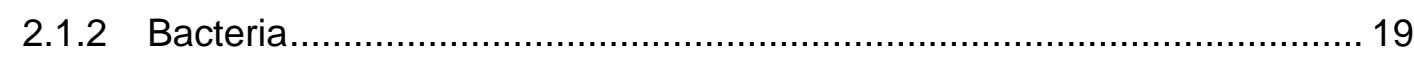

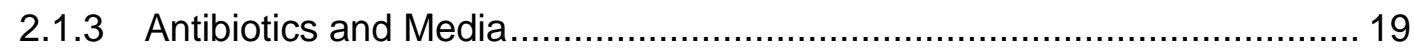

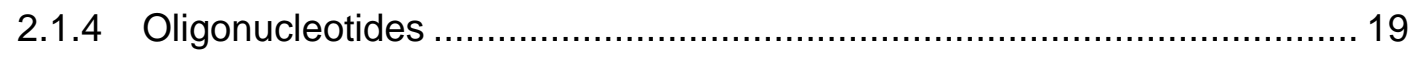

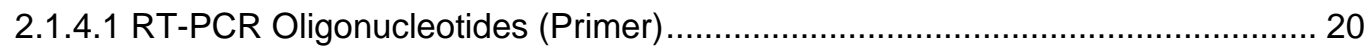

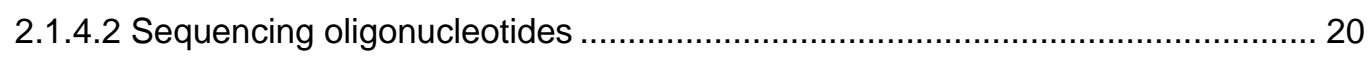

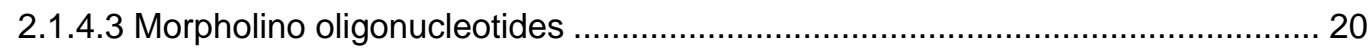

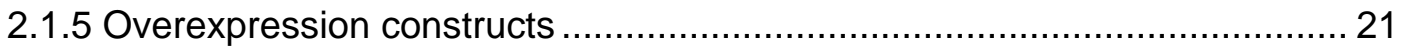




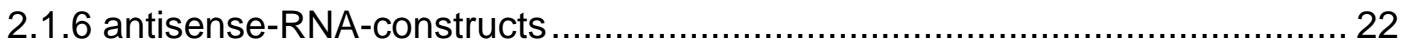

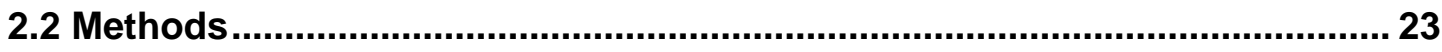

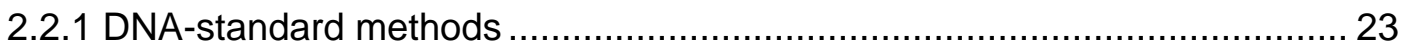

2.2.1.1 Polymerase chain reaction (PCR) ….......................................................... 23

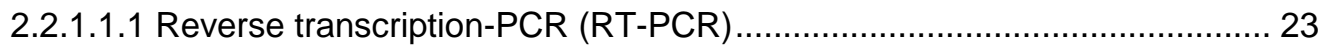

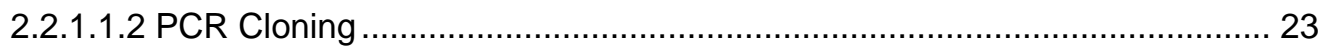

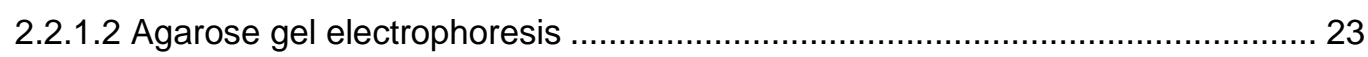

2.2.1.3 Gel purification of PCR and restriction fragments....................................... 24

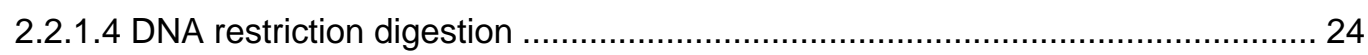

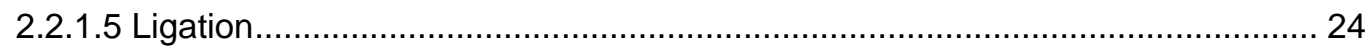

2.2.1.6 Chemical transformation of bacterial cells .................................................... 24

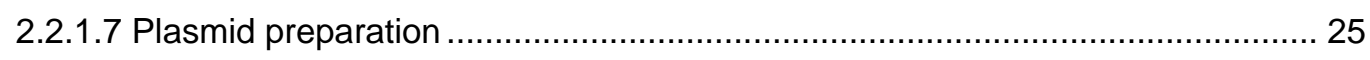

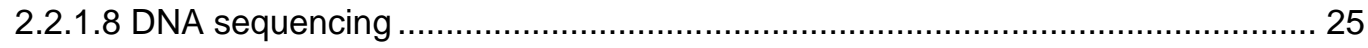

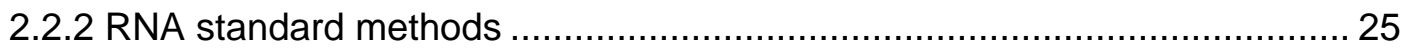

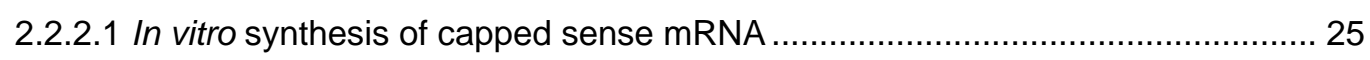

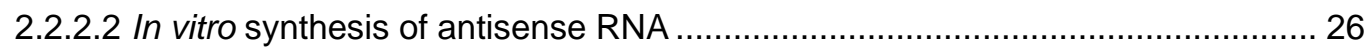

2.2.2.3 Total RNA isolation from ectodermal explants and whole embryos ................. 26

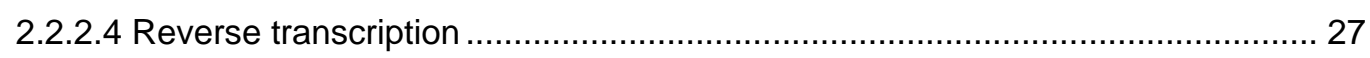

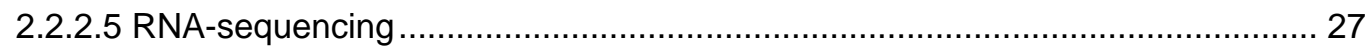

2.2.2.5.1 Total RNA isolation from ectodermal explants ....................................... 27

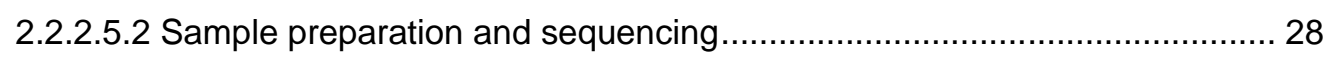

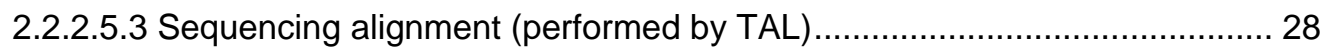

2.2.2.5.4 Statistical analysis (performed by TAL) .................................................. 28

2.2.3 X. laevis embryo culture and micromanipulations .................................... 29

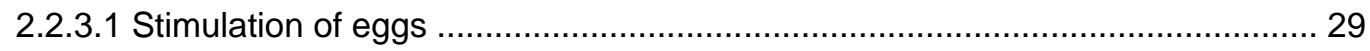

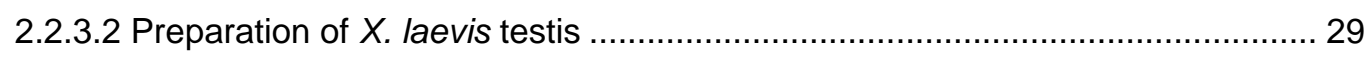

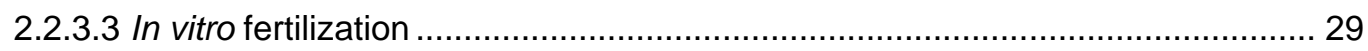

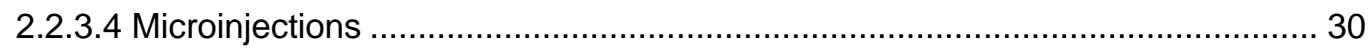

2.2.3.5 X. laevis ectodermal explants ("animal caps") ................................................ 30

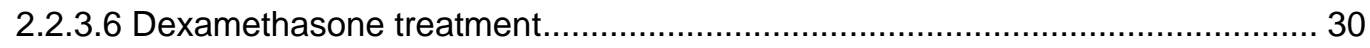

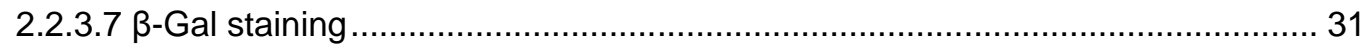

2.2.4 Whole mount in situ-hybridization (WMISH) ..................................... 31

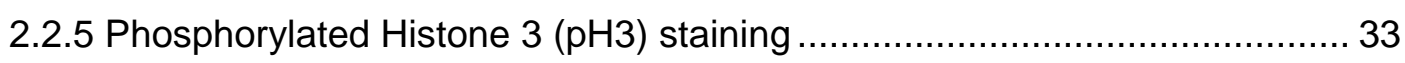

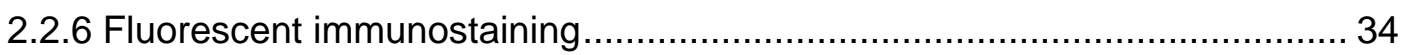

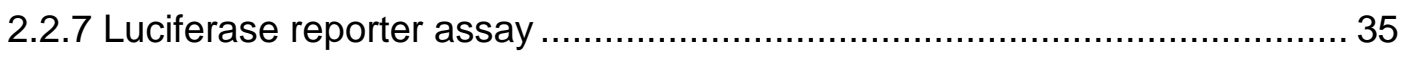

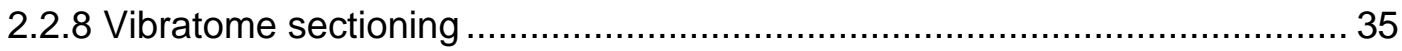

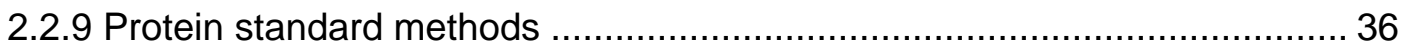

2.2.9.1 SDS-polyacrylamide gelelectrophoresis (SDS-PAGE) …............................. 36

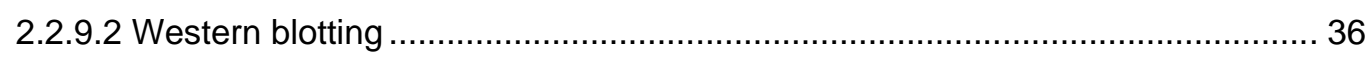

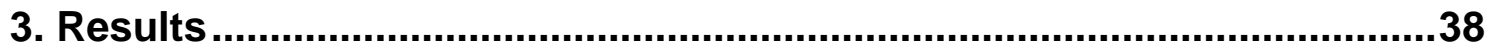


3.1 Temporal and spatial expression analysis of $X$. laevis prdm14.................38

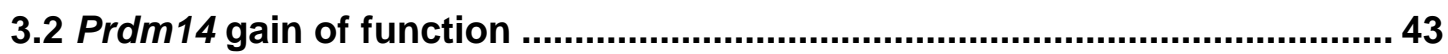

3.3. Prdm14 promotes proliferation and the expansion of neural progenitors 44

3.4 Prdm14 promotes ectopic sensory neuron formation ................................ 46

3.5 Knock down of Prdm14......................................................................... 49

3.6 Identification of Prdm14 downstream targets .......................................... 52

3.7 Prdm14 activates canonical Wnt-signaling.............................................. 56

3.8 Comparative expression analysis of prdm14 with neural plate border

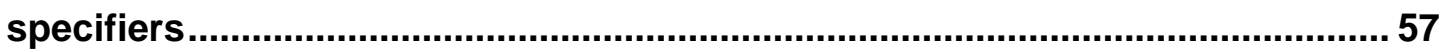

3.9 Knock down of Prdm14 has no influence on candidate gene expression.. 59

3.10 Prdm14 gain of function activates neural crest genes in whole embryos 60

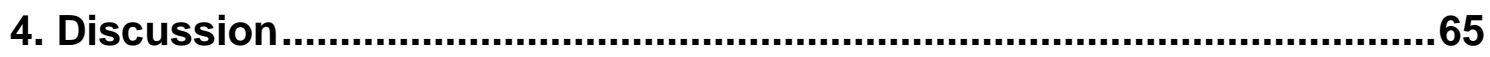

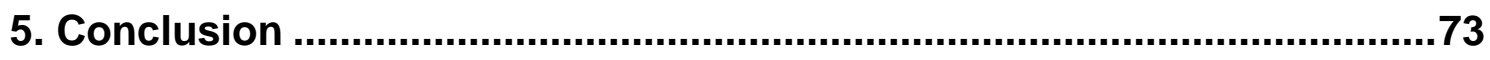

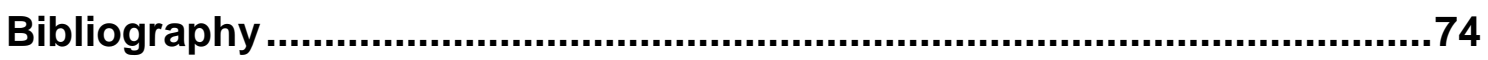

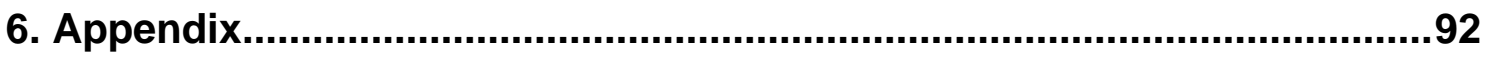

6.1 Candidate gene list for the RNA-sequencing analysis of prdm14-GR overexpressing animal caps ....................................................................... 92

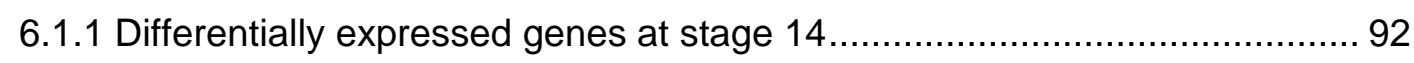

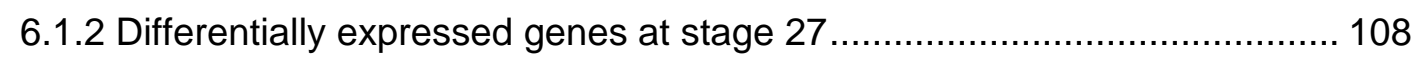

$6.2 \mathrm{GO}$ analysis of candidate genes ............................................................. 118

6.2.1 GO analysis of candidate genes upregulated at stage $14 \ldots \ldots \ldots \ldots \ldots \ldots \ldots . . . . . . . . . .118$

6.2.2 GO analysis of candidate genes downregulated at stage $14 \ldots \ldots \ldots \ldots \ldots . . . . . . .125$

6.2.3 GO analysis of candidate genes upregulated at stage $27 \ldots \ldots \ldots \ldots \ldots \ldots . . . . . . . . . .128$

6.2.4 GO analysis of candidate genes downregulated at stage $27 \ldots \ldots \ldots \ldots \ldots . . . . . .132$ 


\section{Acknowledgements}

First of all, I would like to thank Dr. Kristine Henningfeld. I am very grateful for her supervision, help and patience over the last couple of years. She always supported me and gave advice and encouragement, not only in scientific matters.

I would also like to thank Prof. Dr. Pieler for his help, support and fruitful discussions during the years. Additionally, I thank him for being a member of my examination board on such short notice.

Furthermore, I thank Prof. Dr. Bucher and Prof. Dr. Wodarz for being part of my thesis committee and for their advices and suggestions. I also thank Prof. Dr. Wimmer and Prof. Dr. Mansouri for agreeing to be part of my examination board.

I thank all the members of the Department of Developmental Biochemistry for the great working atmosphere. Additionally, I thank the members of the TAL for their help with the RNA-sequencing. I would like to thank especially Katja Ditter for her technical assistance, Dr. Juliane Melchert, Dr. Marie Hedderich, Dr. Juliane Wellner, Ilona Wunderlich, Anita Smarandache, Maja Gere, Dr. Diana Bauermeister and Sven Richts. Their support and encouragement helped me a lot during the last weeks of finishing this thesis.

I would like to thank my family, and in particular my parents, for their unconditional love and support in everything I did throughout my life.

My special thanks go to my wife, Maren. Without her help, support and comfort during all these years, I would not have made it. She lifted me up, when I was down and was always there, when I needed her. I thank her for her love, her patience and for our son. 


\section{Abstract}

Prdm14 is a member of the conserved family of Prdm proteins, which are emerging as crucial regulators of multiple early developmental processes and diseases. Members of the Prdm family are characterized by the presence of a single PR domain and a variable number of DNA-binding zinc fingers. Most members of the Prdm proteins directly influence transcription through intrinsic histone methyltransferase activity or recruitment of cofactors. In this thesis, a functional analysis of Prdm14 was undertaken to elucidate its role during early vertebrate development using the $X$. laevis model system.

Prdm14 is expressed during gastrulation in the prospective neuroectoderm. At late neural plate stages, the expression is restricted to the territories of primary neurogenesis. In tailbud stage embryos, expression of prdm14 is detected in postmitotic neurons of the central nervous system and a subset of the cranial ganglia. Prdm14 is regulated positively by the proneural bHLH gene neurog2 and negatively by the Notch signaling pathway, which is strongly suggestive for a role during primary neurogenesis. Overexpression of prdm14 in $X$. laevis embryos promotes the proliferation of neural progenitor cells, which results in the expansion of the neural plate and in a transient inhibition of neuronal differentiation. In tailbud stage embryos, prdm14 overexpression induces the formation of ectopic neurons in the non-neural ectoderm that express markers indicative of a sensory glutamatergic neuronal cell fate. In pluripotent animal cap cells, prdm14 overexpression is sufficient to induce a glutamatergic neuronal cell fate supporting the studies in embryos. The downstream transcriptional network induced by prdm14 was studied through RNA-sequencing analysis of prdm14-injected animal caps at the equivalent of neural plate and tailbud stage. Predominant among the prdm14upregulated genes were ligands and signaling components of the Wnt pathway and Wnt-regulated genes. Consistent with the RNA-sequencing analysis, prdm14 overexpression in the embryo activated a canonical Wnt-signaling reporter. In addition, many of the upregulated genes have previously been shown to be involved in neural crest and sensory neuron specification, including the key neural plate border specifiers pax3 and zic1. The activation of these genes may in part be attributed to the ability of prdm14 to activate canonical 
Wnt-signaling. Taken together, the results of this thesis provide evidence for multiple roles of Prdm14 during the development of the nervous system. During gastrulation Prdm14 promotes maintenance of the neural ectoderm and the specification of the neural plate border, which will give rise to neural crest and Rohon-Beard sensory neurons. Also suggested by the expression and regulation of prdm14, is a role during neuronal differentiation and maturation. 


\section{List of Figures}

Figure 1.1 Overview of the establishment of the central nervous 2 system in $X$. laevis.

Figure 1.2 Experimental evidence supporting the default model of 3 neural induction in $X$. laevis.

Figure 1.3 Overview of the molecular mechanisms resulting in neural 5 induction in $X$. laevis.

Figure 1.4 Neuronal subtype specification in $X$. laevis.

Figure 1.5 Delta-Notch pathway mediated-lateral inhibition.

Figure 1.6 Neural crest development in X. laevis.

Figure 1.7 Overview of the protein structure of members of the Prdm family.

Figure 3.1 Temporal and spatial expression analysis of prdm14 in $X$. laevis embryos.

Figure 3.2 Prdm14 is expressed in the marginal zone of the neural 40 tube.

Figure 3.3 Prdm14, neurog1 and neurog2 are co expressed in 41 territories of primary neurogenesis. 
Figure 3.4 Prdm14 expression is regulated by key regulators of 42 neurogenesis.

Figure 3.5 Prdm14-GR overexpression induces stronger phenotypes. 44

Figure 3.6 Prdm14-GR overexpression promotes proliferation.

Figure 3.7 Prdm14-GR overexpression promotes sensory neurons in 47 tailbud stage embryos.

Figure 3.8 Prdm14-GR overexpression activates sensory neuron 48 marker tlx3 in animal caps.

Figure 3.9 Injection of Prdm14 splicing morpholinos causes intron1 50 retention.

Figure 3.10 Knock down of Prdm14 does not influence neuronal 51 differentiation and axon outgrowth of motor neurons.

Figure 3.11 RNA-sequencing analysis of prdm14-GR overexpression in animal caps.

Figure 3.12 Genes of biological processes involved in neural 54 development are enriched in prdm14-GR overexpressing animal caps.

Figure 3.13 Prdm14-GR overexpression activates canonical Wnt- 57 signaling.

Figure 3.14 Prdm14 is co-expressed with pax3 and zic1 in regions of 58 the neural plate border. 
Figure 3.15 Prdm14 knock down does not influence the expression of 60 selected candidate genes in whole embryos.

Figure 3.16 Prdm14-GR overexpression promotes the expression of 61 selected candidate genes in whole embryos.

Figure 3.17 Prdm14-GR overexpression promotes the neural crest 63 formation in whole embryos.

Figure 5.1 Model for the function of Prdm14 in X. laevis. 
List of Tables

Table 1.1 Prdms with known intrinsic HMTase activity.

Table 2.1 Summary of RT-PCR oligonucleotides and their annealing temperatures

Table 2.2 Summary of sequencing oligonucleotides and their 20 annealing temperatures

Table 2.3 Summary of morpholino oligonucleotides

Table 2.4 Summary of antisense RNA constructs

Table 2.5 Summary of antibodies for western blot analysis

Table 3.1 List of selected candidate genes upregulated in prdm14-GR overexpressing animal caps.

Table 6.1 Summary of differentially expressed genes at stage 14

Table 6.2 Summary of differentially expressed genes at stage 27

Table 6.3 Summary of GO analysis of genes upregulated at stage 14

Table 6.4 Summary of GO analysis of genes downregulated at stage 14

Table 6.5 Summary of GO analysis of genes upregulated at stage 27

Table 6.6 Summary of GO analysis of genes downregulated at stage 27 


\section{Abbreviations}

\begin{tabular}{|c|c|}
\hline$A$ & Alanin \\
\hline $\mathrm{AP}$ & alkaline phosphatase \\
\hline aa & amino acid \\
\hline $\mathrm{BCIP}$ & 5-bromo-4-chloro-3-indolyl-phosphate \\
\hline bHLH & basic-helix-loop-helix \\
\hline BMB & Bohringer Mannheim blocking reagent \\
\hline BMP & bone morphogenetic protein \\
\hline bp & base pairs \\
\hline BSA & bovine serum albumin \\
\hline${ }^{\circ} \mathrm{C}$ & degree Celsius \\
\hline $\mathrm{CC}$ & control caps \\
\hline cDNA & complementary DNA \\
\hline CE & control embryos \\
\hline CIAP & calf intestine alkaline phosphatase \\
\hline CoMO & standard control morpholino oligo \\
\hline Dex & dexamethasone \\
\hline Dig & digoxigenin \\
\hline DNA & desoxyribonucleic acid \\
\hline DNAse & desoxyribonuclease \\
\hline DTT & 1,4-dithiothreitol \\
\hline EDTA & ethylendiaminetetraacetic acid \\
\hline EGTA & ethylenglycole-bis(2-aminoethylether)-N,N'- tetraacetate \\
\hline et al. & et alii \\
\hline $\mathrm{EtOH}$ & ethanol \\
\hline FGF & fibroblast growth factor \\
\hline Flu & fluorescein \\
\hline GABA & gamma-aminobutyric acid \\
\hline GR & glucocorticoid receptor \\
\hline $\mathrm{h}$ & hour/hours \\
\hline $\mathrm{H} 4$ & histone 4 \\
\hline $\mathrm{HA}$ & hemagglutination \\
\hline HCG & human chorionic gonadotropin \\
\hline
\end{tabular}




\begin{tabular}{|c|c|}
\hline $\mathrm{H}_{2} \mathrm{O}$ & water \\
\hline is & injected side \\
\hline k & kilo \\
\hline $\mathrm{kb}$ & kilobase \\
\hline I & liter \\
\hline LB & Luria-Bertani \\
\hline$\mu$ & micro \\
\hline $\mathrm{m}$ & milli \\
\hline M & molar \\
\hline MAB & maleic acid buffer \\
\hline MEM & MOPS-EGTA-MgSO 4 buffer \\
\hline MEMFA & MOPS-EGTA-MgSO 4 formaldehyde buffer \\
\hline $\mathrm{MeOH}$ & methanol \\
\hline $\min$ & minutes \\
\hline mRNA & messenger RNA \\
\hline $\mathrm{n}$ & Nano, number \\
\hline $\mathrm{NaAC}$ & sodium acetate \\
\hline NBT & nitro-blue-tetrazolium \\
\hline $\mathrm{nm}$ & nanometer \\
\hline odc & ornithine decarboxylase \\
\hline PAGE & polyacrylamide gel electrophoresis \\
\hline PBS & phosphate buffered saline \\
\hline PCR & polymerase chain reaction \\
\hline $\mathrm{pH}$ & negative decade logarithm of hydrogen ion concentration \\
\hline $\mathrm{pH} 3$ & phosphorylated histone 3 \\
\hline$\%$ & percentage \\
\hline Prdm & $\begin{array}{l}\text { positive regulatory domain l-binding factor1 (PRD1- } \\
\text { BF1/PRDM1) and retinoblastoma-interacting zinc finger } \\
\text { protein } 1 \text { (RIZ1/PRDM2) }\end{array}$ \\
\hline RNA & ribonucleic acid \\
\hline RNase & ribonuclease \\
\hline rpm & rounds per minute \\
\hline RT & room temperature, reverse transcriptase \\
\hline RT-PCR & reverse transcriptase PCR \\
\hline
\end{tabular}




$\begin{array}{ll}\text { SDS } & \text { sodium dodecyl sulfate } \\ \text { sec } & \text { second } \\ \text { SHH } & \text { Sonic Hedgehog } \\ \text { SpMO } & \text { splicing morpholino oligo } \\ \text { SSC } & \text { standard saline citrate buffer } \\ \text { St } & \text { stage } \\ T_{A} & \text { annealing temperature } \\ T_{\text {Taq }} & \text { Thermus aquaticus } \\ \mathrm{T}_{\mathrm{m}} & \text { melting temperature } \\ \mathrm{U} & \text { units } \\ \mathrm{V} & \text { voltage } \\ \text { Vol. } & \text { volume } \\ \text { wmish } & \text { whole mount in situ hybridization } \\ \text { X-Gal } & \text { 5-bromo-4-chloro-3-indolyl- } \beta \text { - } d \text {-galactoside }\end{array}$




\section{Introduction}

\subsection{Xenopus neurogenesis}

The African clawed frog Xenopus is an attractive model organism to elucidate the molecular mechanics of early vertebrate development. The two species mainly used are the allotetraploid Xenopus laevis (X. laevis), and the closely related Xenopus tropicalis ( $X$. tropicalis), which has a diploid and sequenced genome (Amaya, 2005; Geach and Zimmerman, 2011; Grainger, 2012). One of the key advantages of the Xenopus model system is the external development of the embryos, which makes them readily accessible for chemical treatments and microinjections of DNA, mRNA or antisense morpholino oligonucleotides. More recently, the use of gene editing tools like TALENs or CRISPR/Cas have been successfully applied in the Xenopus model system (Lei et al., 2012; Nakayama et al., 2013; Guo et al., 2014; Liu et al., 2014). A relatively simple and yet powerful experimental method is the animal cap assay, in which prospective ectodermal cells of the animal pole are isolated from a blastula stage embryo. These cells are an attractive source for in vitro studies of differentiation since they are pluripotent and able to differentiate into many cell types upon overexpression of different mRNAs (Ariizumi et al., 2009; Borchers and Pieler, 2010).

Xenopus is an important and compelling model organism in studies of early vertebrate neurogenesis, not only due to afore mentioned advantages, but also as the primary neurons are born during the first 24 hours of development (Hartenstein, 1989; Roberts, 2000). These neurons are termed primary neurons and arise in the posterior neural plate in three bilateral longitudinal domains (Chitnis et al., 1995). With the exception of the trigeminal placodes, neuronal differentiation in the anterior neural plate is delayed until late neurula stages (Papalopulu and Kintner, 1996). The primary nervous system is required for touch responses and movements of the early tadpole (Roberts, 2000). Secondary neurogenesis occurs prior to metamorphosis and generates the mature nervous system required for the adult frog (Wullimann et al., 2005). 


\subsection{Neural induction}

One of the earliest events in the development of the vertebrate nervous system is the specification of the dorsal ectoderm into a neural lineage, which occurs through the process of neural induction (Pera et al., 2014). The induced neuroectoderm forms the neural plate and is comprised of mitotically active neural precursor cells separated from the non-neural ectoderm by the neural plate border (Aruga and Mikoshiba, 2011) (Fig. 1.1). The neural plate is a bilayered epithelium with a superficial and a deeper sensorial layer (Hartenstein, 1989; Chalmers et al., 2002). The primary neurons are born in the sensorial layer, while the cells in the superficial layer continue to proliferate and will later give rise to secondary neurons (Hartenstein, 1989; Chalmers et al., 2002; Wullimann et al., 2005). The neural plate will give rise to the brain, spinal cord and retina and derivatives of the neural plate border include the neural crest, neurogenic placodes, Rohon-Beard sensory neurons and the hatching gland (Kuroda et al., 2004; Rossi et al., 2009; Bae et al., 2014).

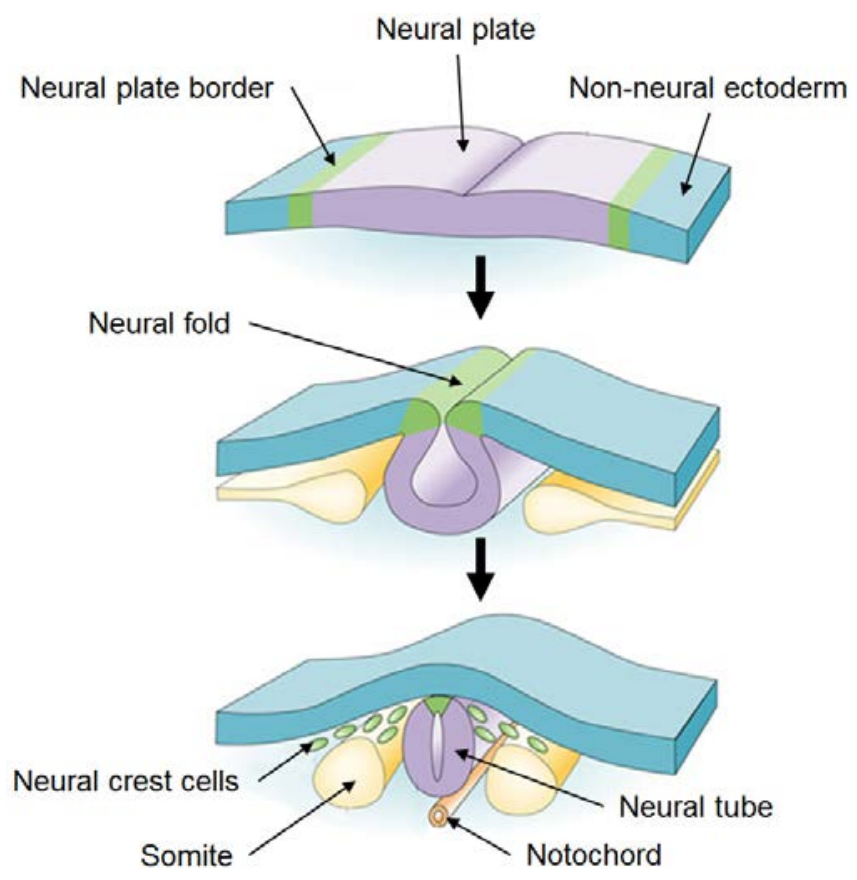

Fig. 1.1 Overview of the establishment of the central nervous system in $X$. laevis. After neural induction the dorsal ectoderm forms the neural plate. The non-neural ectoderm and the neural plate are separated by the neural plate border. After neurulation, the neurons differentiate into distinct subtypes, while the neural plate border contributes to the most dorsal aspect of the neural tube from where neural crest cells, Rohon-Beard sensory neurons and neurogenic placodes arise (Gammill and Bronner-Fraser, 2002; modified). 
Many of the key experiments that essentially contributed to our understanding of vertebrate neural induction have been carried out in $X$. laevis, using transplantation and animal cap assay experiments (Linker and Stern, 2004). One crucial finding was the observation that intact animal cap explants, which normally give rise to atypical epidermis, differentiate into neural tissue upon dissociation (Wilson and Hemmati-Brivanlou, 1995) (Fig. 1.2). This suggested that a soluble inhibitor of the neural fate is present in the animal cap, but is diluted out upon dissociation (Grunz and Tacke, 1989; Sato and Sargent, 1989). This idea was further supported by the neuralization of animal caps upon overexpression of a dominant-negative activin receptor, and the inhibition of a neural fate when dissociated animal cap cells were cultured in the presence of BMP4 (Hemmati-Brivanlou and Melton, 1992; Hemmati-Brivanlou and Melton, 1994; Wilson and Hemmati-Brivanlou, 1995). The sum of these findings led to the postulation of the "default model" of neural induction. In this model, the default state of the ectoderm is thought to be neural and requires the inhibition of epidermal promoting BMP signaling (Hemmati-Brivanlou and Melton, 1997; Stern, 2005; Pera et al., 2014).

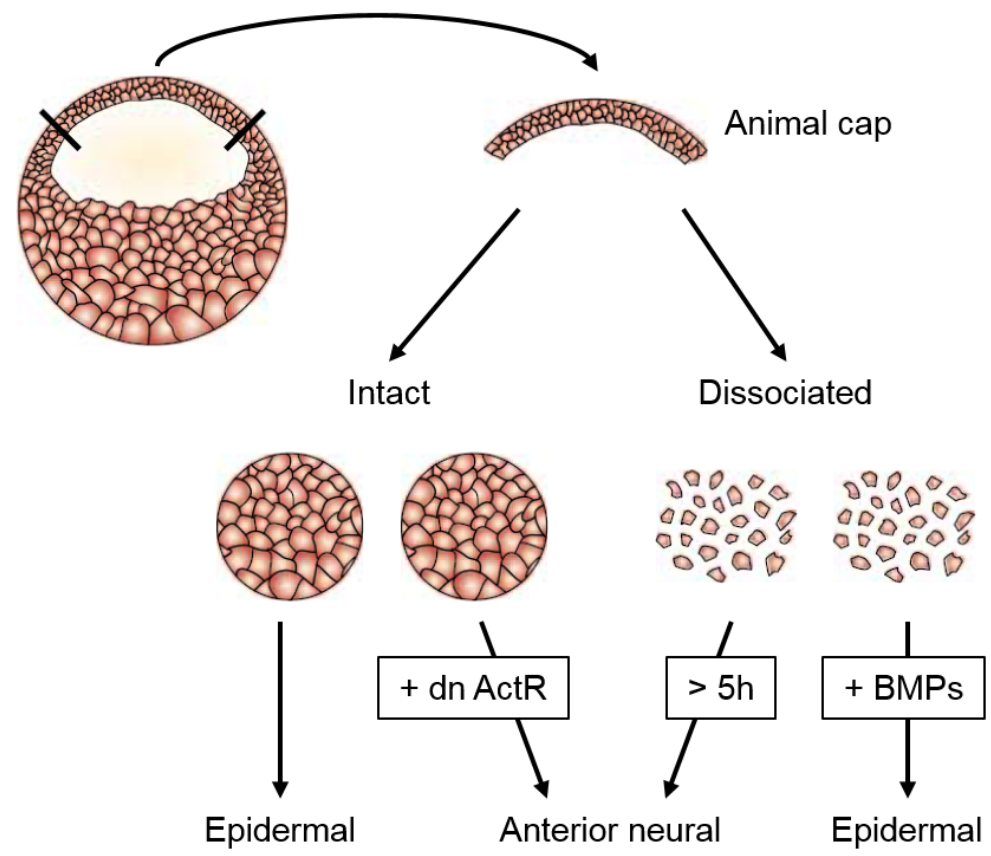

Fig. 1.2 Experimental evidence supporting the default model of neural induction in $X$. laevis. Intact ectodermal explants (animal caps), excised from the animal pole of a blastula stage embryo (stage 8-9) will give rise to atypical epidermis. If this tissue ectopically expresses a dominant-negative activin receptor (dn ActR) or is dissociated, these cells acquire an anterior neural fate. Addition of BMP to dissociated cells will restore epidermal fate (Muñoz-Sanjuán and Brivanlou, 2002; modified). 
The inhibition of BMP signaling can occur on different levels, including extracellular inhibition by factors secreted from different signaling centers (Kuroda et al., 2004; Ozair et al., 2013) (Fig. 1.3). At blastula stages, Chordin (Piccolo et al., 1996; Sasai et al., 1994) and Noggin (Smith and Harland, 1992; Lamb et al., 1993) are secreted from the blastula Chordin- and Nogginexpressing (BCNE) center, which is located in dorsal animal cells (Kuroda et al., 2004). During gastrulation, Chordin, Noggin, Follistatin (Hemmati-Brivanlou et al., 1994) and Cerberus (Bouwmeester et al., 1996; Piccolo et al., 1999) are expressed in the Spemann organizer, which involutes and secretes these BMP antagonists into the overlying prospective neuroectoderm (Aruga and Mikoshiba, 2011).

Despite the multiple lines of evidence supporting the "default model" of neural induction, numerous studies have convincingly challenged this model. In chick embryos, a requirement for FGF signaling prior to BMP antagonism has been demonstrated (Streit et al., 2000). Furthermore, the dissociation of $X$. laevis animal caps triggers the activation of the FGF pathway, while if FGFsignaling is blocked, neuralization of the dissociated animal caps is prevented (Kuroda et al., 2005). Taken together, it is now accepted that inhibition of BMP signaling alone is not sufficient for neural induction but that FGF signaling is also required (Kengaku and Okamoto, 1995; Lamb and Harland, 1995; Streit et al., 2000; Pera et al., 2003; Linker and Stern, 2004, Delaune et al., 2005) (Fig. 1.3). In addition, an involvement of $\mathrm{Ca}^{2+}$-influx in the process of neural induction has also been demonstrated (Moreau et al., 2008; Leclerc et al., 2012). 


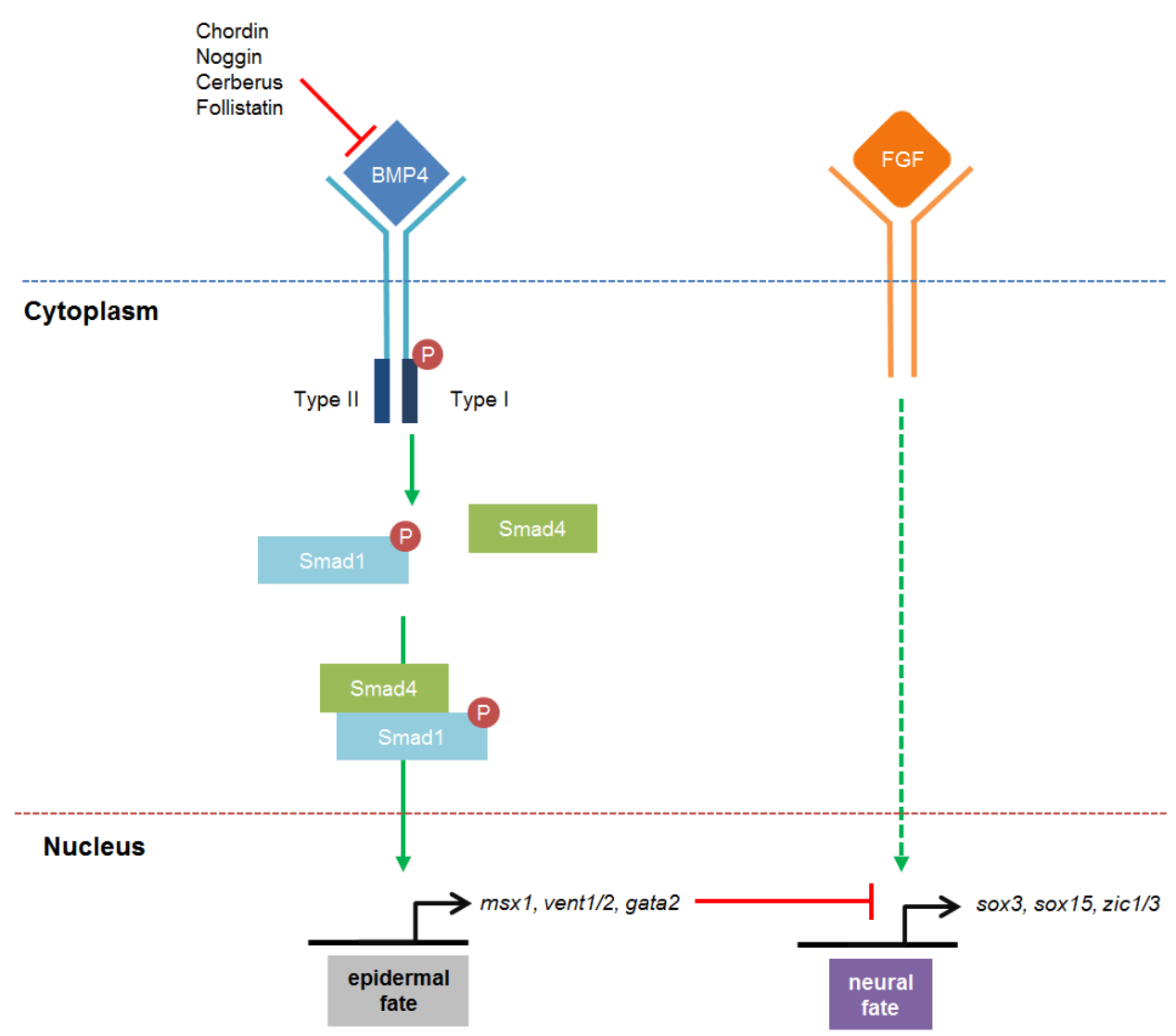

Fig. 1.3 Overview of the molecular mechanisms resulting in neural induction in

$X$. laevis. BMP ligand binding triggers autophosphorylation and dimerization of the receptors, which in turn leads to the phosphorylation of SMAD1. SMAD1 and -4 form a complex and translocate to the nucleus where they activate the expression of epidermal genes such as gata2, msx1 and vent1/2. This activation can be inhibited extracellularly by secreted BMP inhibitors (Chordin, Noggin, Cerberus and Follistatin). Active FGF signaling together with inhibition of BMP cause neuralization of the epidermis by expression of pan-neural genes such as sox3, sox15 and zic1/3.

\subsection{Stabilization of the neural fate}

Neural induction results in the expression of several transcription factors within the neuroectoderm (Sasai, 1998; Moody and Je, 2002) including members of the Sry-related high-mobility group box (sox) family, which are expressed in the neural progenitor cells (Lefebvre et al., 2007). Sox2 and sox3 are early pan-neural genes belonging to the SoxB1 subfamily, which maintain the neural progenitor state (Penzel et al., 1997; Uchikawa et al., 1999; Graham et al., 2003; Pevny and Placzek, 2005). Sox15 (also known as soxd) is also broadly expressed throughout the neural plate and is induced in response to BMP inhibition. Overexpression of sox15 expands the neural plate and promotes a delayed neuronal differentiation (Mizuseki et al., 1998b). Other transcription factors that maintain proliferating neural progenitors are the coiledcoiled protein Geminin (Kroll et al., 1998; Seo and Kroll, 2006; Papanayotou et 
al., 2008) and the forkhead/winged helix transcription factor FoxD5 (Solter et al., 1999; Sullivan et al., 2001).

Members of the zic family of zinc finger transcription factor genes are expressed throughout the neural plate border and are required in early neurogenesis and neural crest formation (Houtmeyers et al., 2013). Overexpression of zic1, 2 and 3 in $X$. laevis results in the expansion of the neural plate and promotes neural crest formation. In addition, zic1 and zic3 both promote neurogenesis upon overexpression (Nakata et al., 1997; Mizuseki et al., 1998a, Rogers et al., 2009). In contrast, zic2, which is also expressed between the territories of primary neurogenesis, inhibits neuronal differentiation (Brewster et al., 1998).

\subsection{Neuronal differentiation}

In $X$. laevis the primary neurons are born in three longitudinal bilateral domains in the sensorial layer of the posterior neural plate and the anteriorly located trigeminal placodes (Papalopulu and Kintner, 1996) (Fig. 1.4A). In these territories of primary neurogenesis, the first genes expressed are proneural basic helix-loop-helix (bHLH) transcription factor gene of the neurogenin family (Imayoshi and Kageyama, 2014). Members of this family are transcriptional activators that heterodimerize with more broadly expressed $E$ proteins and bind within the regulatory domains of their target genes at $E$ box motifs (CANNTG) (Bertrand et al., 2002). These proneural genes trigger general neurogenesis and promote the differentiation of specific neuronal and/or glial cells, which suggest a common set of target genes for generic neurogenesis but an individual set of target genes for distinct neuronal subtypes (Powell and Jarman, 2008).

The neurogenins are orthologs of the Drosophila neuronal determination factor atonal (Ma et al., 1996; Nieber et al., 2009) and exhibit a proneural activity by inducing downstream proneural transcription factors and regulating cell-cycle exit (Ma et al., 1996; Bertrand, 2002; Souopgui et al., 2002; de la Calle-Mustienes et al., 2002). The neurogenins are already expressed during early gastrulation, prefiguring the domains of primary neurons in the deep layer of the prospective neuroectoderm. The expression of neurog1 and neurog 2 is 
present in all three longitudinal stripes of primary neurogenesis with neurog2 being expressed much broader (Ma et al., 1996; Nieber et al., 2009). Neurog3 has a much more restricted expression domain that encompasses the medial domain (Nieber et al., 2009).

Neurog2 is the best characterized proneural gene in $X$. laevis and its expression is induced by the pre-pattern genes zic1, zic3 and the pan-neural gene sox15, whereas zic2 represses the expression and function of neurog1 and neurog2 (Mizuseki et al., 1998a; Mizuseki et al., 1998b, Rogers et al., 2009). Neurog 2 induces the expression of several neuronal differentiation factor genes such as for the transcription factors neurod1 (Ma et al., 1996), neurod4 (Perron et al., 1999), ebf2 (Dubois et al., 1998), ebf3 (Pozzoli et al. 2001), myt1 (Bellefroid et al., 1996), mtgr1 (Koyano-Nakagawa and Kintner, 2005), hes6 (Koyano-Nakagawa et al., 2000), the RNA binding protein seb4r (Boy et al., 2004), as well as the Notch ligands dll1 and dll4 (Chitnis et al., 1995; Bray, 2006). Neurog 2 additionally induces the expression of the cell cycle inhibitor genes gadd45- $y$ and pak3, which enables a proliferating cell to exit the cell cycle and start to differentiate (Souopgui et al., 2002; de la Calle-Mustienes et al., 2002). Surprisingly, the cdk inhibitor gene cdknx is required for neuronal differentiation by promoting the stabilization of Neurog2 (Vernon and Philpott, 2003; Nguyen et al., 2006).

\subsection{Neurulation and neuronal subtype specification}

During neurulation, the neural plate folds and gives rise to the neural tube. The posterior neural tube forms the spinal chord, in which distinct neuronal subtypes are specified depending on their dorso-ventral position (Hartenstein, 1989; Roberts, 2000) (Fig. 1.4). In the closed neural tube, the neural precursor cells are located in the inner ventricular zone (Fig. 1.4B). As neural cells exit the cell cycle, they start to migrate outwards through the subventricular zone into the marginal zone where they differentiate into mature neurons (Taverna and Huttner, 2010; Spear and Erickson, 2012; Miyata et al., 2014). The dorso-ventral patterning of the neurons is the consequence of two opposing morphogen gradients, BMP generated in the dorsal roof plate and sonic hedgehog $(\mathrm{SHH})$ secreted from the ventral floor plate (Le Dreau and 
Marti, 2012). This dorso-ventral morphogen gradient leads to the specification of distinct progenitor domains within the ventricular zone of the neural tube, which are defined by the expression of specific bHLH and homeodomain transcription factors (Jessell et al., 2000; Alaynick et al., 2011). The differentiating cells start to express a unique combinatorial code of transcription factors specific for its subtype and begin to migrate outwards through the subventricular zone into the marginal zone where the cells terminally differentiate into distinct populations of neurons (Ge et al., 2006; Powell and Jarman, 2008; Alaynick et al., 2011).

A

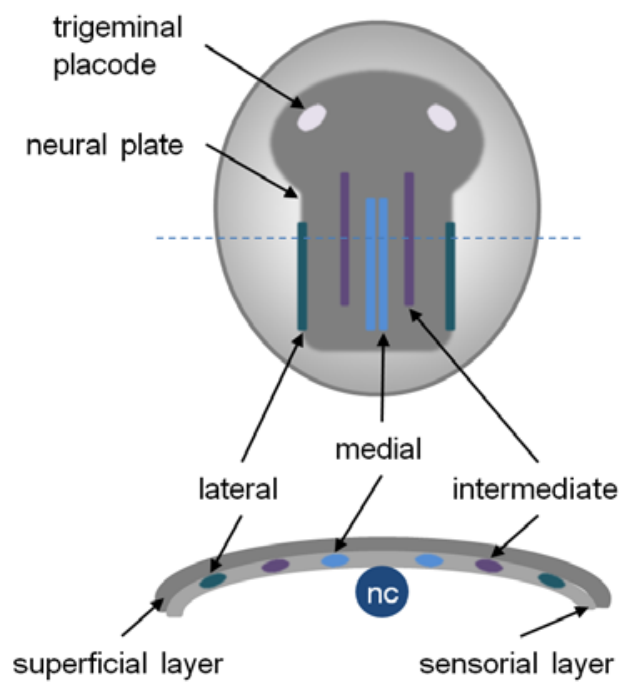

B

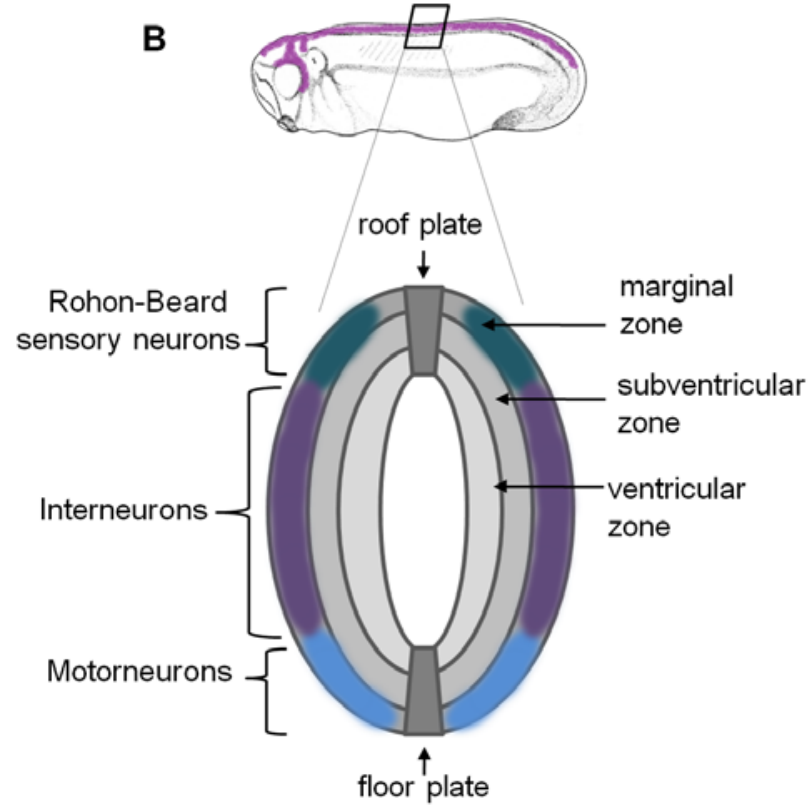

Fig. 1.4 Neuronal subtype specification in X. laevis. (A) In the open neural plate primary neurons are born in the sensorial layer of the neural plate bilaterally in three longitudinal stripes and in the trigeminal placodes. After neurulation, the primary neurons differentiate into specific neuronal subtypes depending on their position within the neural plate and subsequently on their dorso-ventral position within the closed neural tube. Dashed blue line indicates level of section. Nc, notochord (B) Neurons in the lateral stripes will give rise to Rohon-Beard sensory neurons, while neurons in the intermediate stripe differentiate into interneurons. Neurons that are located in the medial stripe will become motorneurons. In the closed neural tube, the neural progenitor cells are located in the ventricular zone where Notch signaling is high. Beginning with differentiation, the cells migrate through the subventricular zone into the marginal zone where they terminally differentiate and mature.

The first neurons are born at mid-gastrula stages and are the laterally located Rohon-Beard mechanosensory neurons (Lamborghini et al., 1980; Rossi et al., 2009) (Fig. 1.4A). These neurons are responsible for touch responses of Xenopus and zebrafish embryos and arise from the neural plate border, which is exposed to intermediate levels of BMP signaling and contributes to the most dorsal aspect of the neural tube (Rossi et al., 2008; 
Rossi et al., 2009, Groves and LaBonne, 2014). With a slight delay, the ventrally located neurons differentiate into motorneurons and Kolmer-Agdhur cells. The latter are cells of unknown function but are speculated to be primitive sensory cells (Hartenstein, 1993; Djenoune et al., 2014). The last neurons to differentiate are the interneurons, which can be subdivided into seven different subtypes (Hartenstein, 1993; Roberts et al., 2012).

\subsection{Lateral inhibition}

The neural plate is comprised of a homogenous population of equipotent proliferating cells (Beatus and Lendahl, 1998). Through the activity of proneural factors such as Neurog2 and Ascl1, lateral inhibition is triggered. This leads to the inhibition of neuronal differentiation in neighbouring cells, resulting in a "saltand-pepper" like pattern of postmitotic neurons (Chitnis et al., 1995; Lewis, 1996; Vasconcelos and Castro, 2014) (Fig. 1.5A). The process of lateral inhibition is mediated by Notch signaling between two neighboring cells. In the signal sending cell that is destined to differentiate into a neuron, the proneural transcription factors induce the expression of transmembrane Notch ligands such as dIll and 4 as well as jag1 in X. laevis (Kiyota and Kinoshita, 2002; Bray, 2006). The Notch ligands then bind to the Notch1-receptor on a neighboring signal receiving cell (Chitnis et al., 1995; Bray, 2006), thereby inducing a series of proteolytic processes within the signal receiving cell that leads to the cleavage of the intracellular domain of the Notch receptor (NICD) (Schroeter et al., 1998; Selkoe and Kopan, 2003). NICD translocates into the nucleus, where it displaces a co-repressor of Rbp-j and acts as a co-activator inducing the expression of genes of the hairy and enhancer of split related (hes) family (Wettstein et al., 1997). These direct Notch target genes are bHLH transcription factor repressor proteins, which inhibit the expression and activity of proneural genes such as neurog2 in the signal receiving cell (Dawson et al., 1995; Wettstein et al., 1997; Li and Baker, 2001; Schneider et al., 2001; Cau et al., 2002; Louvi and Artavanis-Tsakonas, 2006). Hence, the signal sending cell differentiates into a neuron, whereas the neighboring signal receiving cell is prevented from differentiating into a neuron and remains in a proliferating undifferentiated state to maintain a neural progenitor pool (Dawson et al., 1995; Wang and Barres, 2000). Besides its role in lateral inhibition in the neural plate, 
the Notch signaling pathway is also required for the maintenance of a progenitor pool of proliferating neurons in the ventricular zone of the neural tube (Lindsell et al., 1996; Imayoshi and Kageyama, 2011).

The selection process that determines which cell from a pool of equipotent progenitors will differentiate into a neuron is not fully understood (Goodfellow et al., 2014). It has been shown that Neurog2 activates the zinc finger transcription factor gene myt1, which enables a cell to escape lateral inhibition (Bellefroid et al., 1996). Furthermore, the oscillating expression of proneural genes in neural progenitor cells, contributes to the determination of which cells differentiate into neurons (Hatakeyama and Kageyama, 2006; Nelson and Reh, 2008; Kageyama et al., 2008, Goodfellow et al., 2014). In mouse, the oscillating expression of neurog 2 and dll1 is inverse to the Notchmediated induction of hes1, which is an inhibitor of the proneural factors (Shimojo et al., 2008) (Fig. 1.5B). Because of this oscillation, neurog2 expression in a cell does not necessarily result in the differentiation of the cell into a neuron, but requires a certain threshold of neurog2 to be reached and maintained (Kageyama et al., 2008, Goodfellow et al., 2014). The expression of hes1 is regulated by autorepression as well as mRNA and protein instability (Hirata et al., 2002; Momiji and Monk, 2009). The hes1 mRNA is subject to degradation by miR-9, which itself is repressed by Hes1 (Bonev et al., 2012). By this double-negative feedback loop, the expression of hes1 oscillates (Bonev et al., 2012). As the mature miR-9 is only slowly degraded, it accumulates in the cell, which leads to increased degradation of hes1 mRNA. Thus, the proneural factors are released from repression and the cell differentiates into a neuron (Bonev et al., 2012; Goodfellow et al., 2014). 

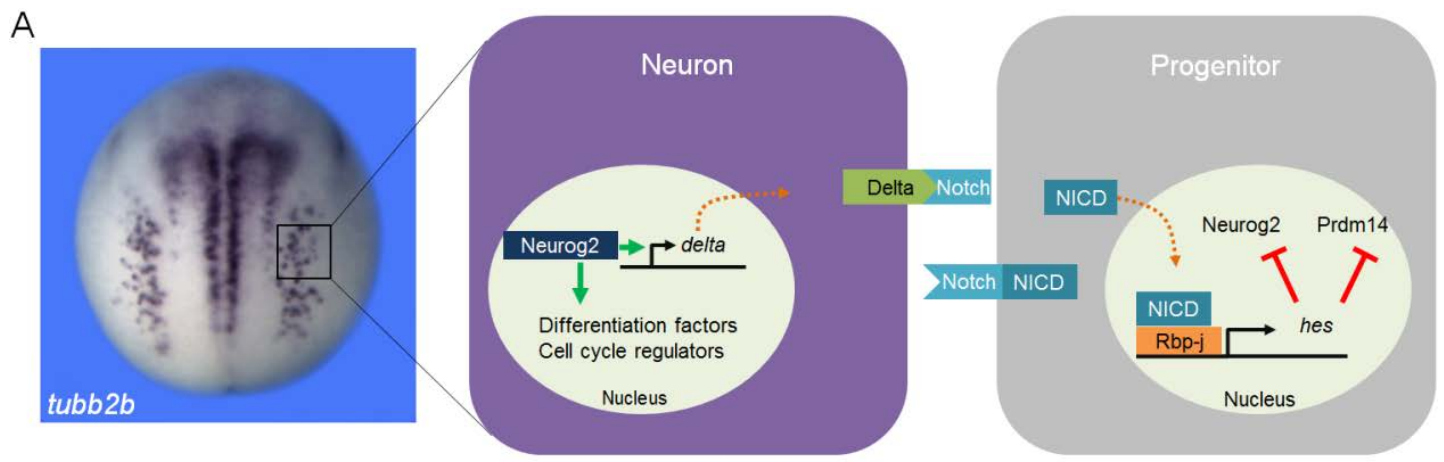

B

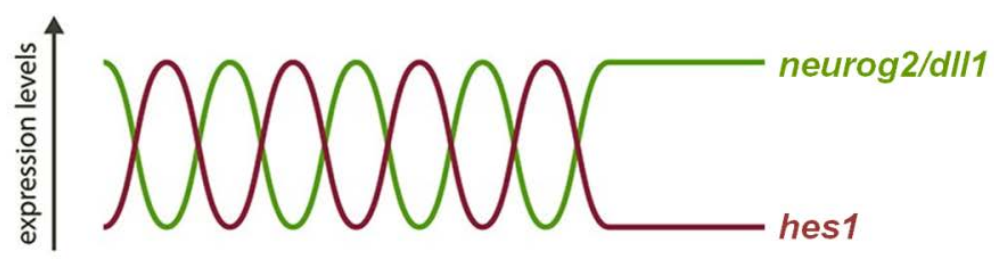

neural stem / progenitor cell $>$ neuron

Fig. 1.5 Delta-Notch pathway mediated-lateral inhibition. (A) In the cell that is destined to differentiate into a neuron, Neurog2 activates the expression of dll1, which translocates to the membrane and interacts with the membrane bound Notch receptor on the signal receiving neighboring cell. In the signal receiving cell, the intracellular domain of Notch (NICD) is released by proteolytic cleavages and translocates to the nucleus. Together with the transcription factor Rbp-j, NICD activates the expression of transcription factors of the Hes-family, which inhibit the expression and function of proneural factors and therefore inhibit neurogenesis. (B) The expression of hes 1 and neurog2 show an inverse oscillation. If hes 1 expression is extinguished, neurog2 expression is sustained, which leads to the differentiation of neural progenitor cells into neurons in a "salt-and-pepper" like pattern (Vasconcelos and Castro, 2014; modified).

\subsection{Neural crest}

The neural crest is a multipotent population of cells that arises from the neural plate border and is unique to vertebrates (Gammil and Bronner-Fraser, 2003) (Fig. 1.6). It is characterized by its high migratory potential and the ability to give rise to multiple derivatives that derive from three different neural crest subpopulations, the cranial-, trunk- and vagal neural crest, which are located along the anterior-posterior axis (Simões-Costa and Bronner, 2015). The cranial neural crest cells contribute to the facial skeleton and neurons of the cranial sensory ganglia (Couly et al., 1998), whereas the vagal neural crest cells form the outflow tract of the heart and enteric ganglia of the gut (Le Douarin and Teillet, 1973; Creazzo et al., 1998). In the peripheral nervous system, the dorsal root and sympathetic ganglia are formed by the trunk neural crest (Le Douarin 
and Smith, 1988). The melanocytes arise from neural crest cells of all three subpopulations (Le Dourain et al., 2004). Upon neural tube closure, the trunk neural crest cells contribute to the most dorsal aspect of the neural tube before they start the process of migration and differentiation (Le Dourain et al., 2004).

The formation of the neural crest occurs during gastrulation and is a multi-step process, which can be divided into neural plate border induction followed by neural crest specification, migration and differentiation (Pegoraro and Monsoro-Burq, 2012; Simões-Costa and Bronner, 2015) (Fig. 1.6A). The induction of the neural plate borders requires intermediate levels of BMP as well as Wnt, FGF and Notch signaling (Endo et al., 2002; Monsoro-Burq et al., 2003; Yardley and Garcia-Castro, 2012; Groves and La Bonne, 2014). The combinatory effects of these signaling pathways result in the expression of several genes within the neural plate border, which form a synexpression group that cross-regulates each other's expression (Nikitina et al., 2008; Bhat et al., 2013). Among these neural plate border specifiers are tfap2, $m s x 1$, zic1, gbx2, pax3/7, dlx5/6, hairy2, c-myc, gata2/3 and foxi1/2 (Bellmeyer et al., 2003; Meulemans and Bronner-Fraser, 2004; Monsoro-Burq et al., 2005; Nichane et al., 2008; Khudyakov and Bronner-Fraser, 2009; de Croze et al., 2011). Pax3 together with zic1 is sufficient, to promote the expression of the neural crest specifiers in animal cap cells (Milet et al., 2013). In contrast, pax3 alone promotes the formation of the hatching gland, while zic1 alone induces a preplacodal ectodermal fate (Hong and Saint-Jeannet, 2007; Bae et al., 2014). The activity of the neural plate border specifiers results in the specification and migration of neural crest cells by inducing the expression of foxd3, snai2 and sox8/9/10 (Dottori et al., 2001; Luo et al., 2003; Cheung et al., 2005; MonsoroBurq et al., 2005; Sato et al., 2005; Nichane et al., 2008; Coles et al., 2007). 


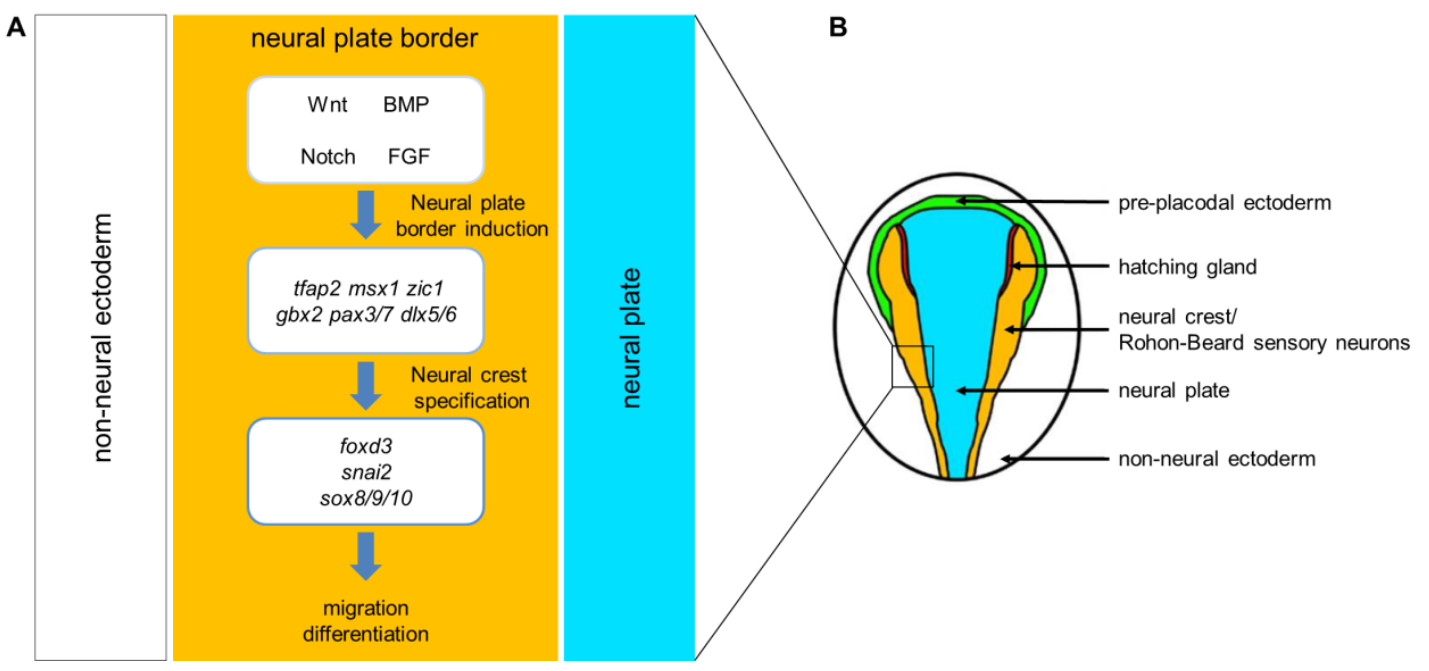

Fig. 1.6 Neural crest development in X. laevis. (A) Through the combinatory activity of Wnt, Notch, BMP and FGF signaling, the neural plate border inducing genes are activated. These genes mutually promote themselves and activate the neural crest specifying genes, that leads to the migration and differentiation of the neural crest cells (Pegoraro and Monsoro-Burq, 2012; modified). (B) Schematic illustration of a neurula stage embryo (dorsal view, anterior to top). Highlighted are the positions of the neural plate, and the neural plate border with its derivatives (pre-placodal ectoderm, hatching gland, neural crest, Rohon-Beard sensory neurons) (Hong and Saint-Jeannet, 2007; modified).

\subsection{The Prdm protein family}

Early vertebrate neurogenesis relies on multiple transcription factors and signaling pathways to ensure the proper establishment of the developing nervous system with its distinct neuronal and glial subtypes. In recent years, the Prdm proteins have emerged as critical players in neuronal subtype specification and neural crest formation (Hohenauer and Moore, 2012). Prdms belong to a highly conserved protein family whose members are involved in several cellular functions including the maintenance of stemness in embryonic stem cells and early developmental processes (Hohenauer and Moore, 2012). Deregulation of Prdms has also been implicated in several human diseases including cancer (Fog et al., 2012).

\subsubsection{Structural properties of Prdm proteins}

Prdms are chromatin modifying proteins characterized by the presence of an N-terminally located PR-domain (Fog et al., 2012) (Fig. 1.7). The PR-domain is similar to the SET (S $u p p r e s s o r$ of variegation 3-9, Enhancer of zeste and trithorax) domain, found in several histone lysine methyltransferases (Huang, 
2002). Correspondingly, several Prdm members have been shown to harbor intrinsic HMTase-activity (Fog et al., 2012; Hohenauer and Moore, 2012). The methylation occurs on different lysine residues, which can result in transcriptionally silenced (Prdm2, 3, 6, 8, 16) as well as active (Prdm9) chromatin states (Table1.1) (Kim et al., 2003; Wu et al., 2008; Eom et al., 2009; Hayashi et al., 2005; Bellefroid, unpublished; Hanotel et al., 2014; Pinheiro et al., 2012). However, a systematic analysis of the putative HMTase activity of other Prdms has not been carried out to date. Interestingly, the conserved motif, which is essential for the histone methylation on lysine by most SET proteins $(\mathrm{H} / \mathrm{R} x x \mathrm{NH} x \mathrm{C})$, is absent in the PR-domain (Rea et al.; 2000). While not all PRDMs show intrinsic HMTase-activity, several have been shown to recruit histone-modifying co-factors such as histone methyltransferases, deacetylases and acetyltransferases (Kouzarides, 2007; Fog et al., 2012; Hohenauer and Moore, 2012).

\begin{tabular}{lll}
\hline Gene & $\begin{array}{l}\text { Intrinsic enzyme } \\
\text { activity }\end{array}$ & References \\
\hline Prdm2 & H3K9me2 & Kim et al., 2003 \\
\hline Prdm3 & H3K9me1 & Pinheiro et al., 2012 \\
\hline Prdm6 & H4K20 & Wu et al., 2008 \\
\hline Prdm8 & H3K9me2 & Eom et al., 2009 \\
\hline Prdm9 & H3K4me3 & Hayashi et al., 2005 \\
\hline Prdm12 & Unknown & Bellefroid, unpublished \\
\hline Prdm13 & Unknown & Hanotel et al., 2014 \\
\hline Prdm16 & H4K9me1 & Pinheiro et al., 2012 \\
\hline
\end{tabular}

Table 1.1 Prdms with known intrinsic HMTase activity. Histone modifications depicted in red promote heterochromatin formation, while histone modifications shown in green represent a transcriptionally active euchromatin state.

With the exception of Prdm11, all members feature a variable number of $\mathrm{C} 2 \mathrm{H} 2$ zinc fingers in their C-terminus(Fog et al., 2012; Hohenauer and Moore, 2012) (Fig. 1.7). Through the zinc fingers, Prdms can bind to DNA and consensus binding sites that have been characterized for Prdm1, 3, 5, 9, 14 and 16 (Delwel et al., 1993; Funabiki et al., 1994; Kuo and Calame, 2004; Duan et 
al., 2007; Seale et al., 2007; Baudat et al., 2010; Chia et al., 2010; Ma et al., 2011; Bard-Chapeau et al., 2012).
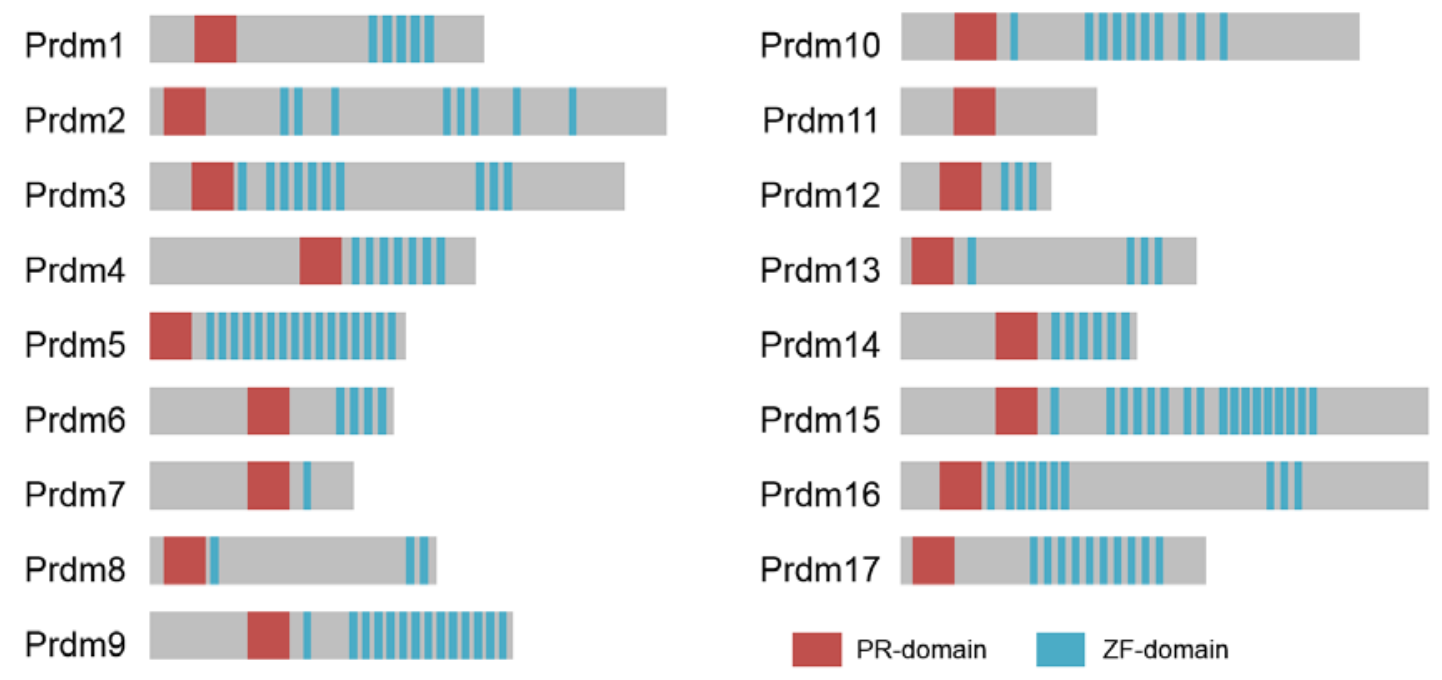

Fig. 1.7 Overview of the protein structure of members of the Prdm family. Shown is the longest isoform of each Prdm protein. The red box depicts the PR-domain and the blue bars represent zinc fingers. The structure is based on the mouse sequence.

\subsubsection{The role of Prdms in early vertebrate neurogenesis and neural crest formation}

Several members of the prdm family are expressed in the developing nervous system of vertebrates (Kinameri, et al., 2008; Sun et al., 2008; Liu et al., 2012, Eguchi, et al., 2015). Functional analyses of individual prdms have been carried out in various model systems and several have been shown to be involved in the specification of distinct neuronal subtypes and the specification of the neural crest (Hernandez-Lagunas et al., 2011; Ding et al., 2013; Hanotel et al., 2014).

\subsubsection{Prdms in neural crest formation}

In zebrafish as well as in $X$. laevis, prdm1 is expressed at the neural plate border and essential for the migration of the trunk neural crest cells and the formation of Rohon-Beard sensory neurons (Hernandez-Lagunas et al., 2005; Rossi et al., 2008; Rossi et al., 2009; Olesnicky et al., 2010; HernandezLagunas et al., 2011; Powell et al., 2013). In zebrafish it has been shown that 
prdm1a is coexpressed with the neural crest specifiers foxd3 and tfap2a, which are directly regulated by Prdm1a (Powell et al., 2013). Furthermore, Prdm1 regulates the expression of sox10 and islet1, which are required for the specification of the neural crest and the formation of Rohon-Beard sensory neurons (Olesnicky et al., 2010; Hernandez-Lagunas et al., 2011). The expression of prdm1 in the neural plate border of the chick embryo was recently reported, suggesting a conservation of function (Zwane and Nikitina 2015).

\subsubsection{Regulation of neuronal subtype specification by Prdms}

Prdm12 has been shown to play a role in neuronal subtype specification in $X$. laevis and zebrafish. In the developing spinal cord, prdm12 promotes the formation of V1-interneurons at the expense of V0-neurons (Bellefroid, unpublished; Zannino et al., 2013). In addition, $X$. laevis prdm12 is also required for the specification of sensory neurons as it regulates the expression of transcriptions required for sensory neuron formation such as sncg, islet1 and tlx3 (Nagy et al., 2015).

In mouse and $X$. laevis, prdm13 is a target of the Ptfla-Rbp-J complex and responsible for the promotion of a GABAergic over a glutamatergic fate of neurons (Chang et al., 2013; Hanotel et al., 2014). Prdm13 promotes a GABAergic neural fate through the inhibition of the glutamatergic selector gene tlx3 (Chang et al., 2013; Hanotel et al., 2014).

\subsubsection{Prdms in axon outgrowth}

In the mouse spinal cord, prdm8 is first expressed in the progenitor populations of ventral interneurons and motor neurons (Komai et al., 2009). Together with the transcription factor Bhlhb5 (also known as Bhlhe22), Prdm8 forms a repressor complex to repress cdh11, whose downregulation is required for proper axon outgrowth of corticospinal motor neurons (Ross et al., 2012).

Prdm14 is required for proper motorneuron axon outgrowth in zebrafish. Loss of Prdm14 through mutation or injection of a splicing morpholino leads to shortened axons in caudal primary (CaP) motor neurons, which in turn results in defective embryonic movement. It has been shown that Prdm14 binds to the 
promoter region of the transcription factor gene islet2 and thus activates its expression, which is necessary for proper CaP axon outgrowth (Liu et al., 2012).

\subsubsection{Prdm14 is an epigenetic regulator and stemness factor}

While in zebrafish a function for prdm14 has been described for motorneuron axon outgrowth, prdm14 in mice and humans is described in the context of primordial germ cell specification and stemness maintenance (Fog et al., 2012; Liu et al., 2012; Nakaki and Saitou, 2014). Prdm14 harbors a single PR-domain and six zinc finger domains that are highly conserved in vertebrates, while the N-terminus of the protein is more divergent (Nakaki and Saitou, 2014). Through its zinc finger domains, Prdm14 can bind and regulate its target genes (Ma et al., 2011). Through ChIP-seq analysis in mouse and human ESC (hESC/mESC) the consensus binding sequence has been identified as 5'GGTCTCTAA-3' (Yamaji et al., 2013). Unlike other Prdms, no intrinsic HMTase activity has been demonstrated for Prdm14. However, in mammalian cell cultures it has been shown that Prdm14 regulates pluripotency and epigenetic reprogramming (Nakaki and Saitou, 2014). In mESCs Prdm14 interacts with the H3K27me3-ase PCR2, which leads to the repression of fgfr1 and fgfr2 and thereby to an inhibition of differentiation (Grabole et al., 2013; Yamaji et al., 2013). Furthermore, the Prdm14/PCR2 complex represses expression of the de novo methyltransferases dnmt3a, $d n m t 3 b$ and $d n m t 3 l$, which leads to low levels of methylation in mESC (Grabole et al., 2013). Through complexation with TenEleven Translocation (TET) proteins, Prdm14 promotes the demethylation of germline specific promoters by oxidation of 5-methylcytosine $(5 \mathrm{mC})$ to 5 hydroxymethylcytosine (5hmC) (Hacket et al., 2013; Okashita et al., 2014).

Besides its repressing properties, Prdm14 is able to activate the expression of genes that are required to maintain pluripotency such as sox 2 or klf5 and in a complex with PRMT4, Prdm14 activates its target genes in mESC through H3 arginine 26 dimethylation (Ma et al., 2011; Burton et al., 2013; Chan et al., 2013; Yamaji et al., 2013; Nakaki and Saitou, 2014). Thus, regulation of Prdm14 target genes is context dependent and Prdm14 can act as an activator as well as a repressor (Ma et al., 2011; Nakaki and Saitou, 2014). 
It is suggested that Prdm14 is involved in the core pluripotency circuitry in hESC were it binds to the proximal enhancer of oct4 and activates its expression (Chia et al., 2010). Similar to mESCs, Prdm14 occupies several target genes together with Oct4, Sox2 and Nanog (Chia et al., 2010). However, prdm14 itself is also occupied by these three factors (Boyer et al., 2005; Chia et al., 2010).

Prdm14 is also required together with Prdm1 for the formation of primordial germ cells in mice (PGCs) by regulating three critical steps: repression of the somatic mesodermal program, reacquisition of potential pluripotency by sox2 activation and epigenetic reprogramming through genomewide DNA demethylation (Ohinata et al., 2005; Yamaji et al., 2008; Ohinata et al., 2009).

\subsection{Aims}

In recent years, the Prdm proteins have emerged as critical transcriptional regulators involved in stemness maintenance, differentiation and epigenetic modification (Fog et al., 2011; Hohenauer and Moore, 2012). In addition, several members of the prdm family play crucial roles in the development of the neural crest and central nervous system (HernandezLagunas et al., 2005; Rossi et al., 2008; Rossi et al., 2009; Olesnicky et al., 2010; Hernandez-Lagunas et al., 2011; Powell et al., 2013, Liu et al., 2012; Zannino et al., 2013; Hanotel et al., 2014; Nagy et al., 2015). In a screen for target genes repressed by the Notch pathway effector protein Hes5.1, prdm14 was identified in X. laevis (Klisch, 2006). Owing to the key role members of this family have during early development, a functional analysis of prdm14 using the $X$. laevis model system was undertaken. Specifically, the developmental processes regulated by prdm14 are to be investigated as well as the downstream gene regulatory network. 


\section{Material and Methods}

\subsection{Material}

\subsubsection{Model organism}

During this study the South African clawed frog Xenopus laevis was used as a model system using animals obtained from Nasco (Ft. Atkinson, USA). The animals were kept according to the directive 2010/63/EU on the protection of animals used for scientific purposes. The developmental stages were determined according to Nieuwkoop and Faber (1967).

\subsubsection{Bacteria}

The chemical competent Escherichia coli-Strain XL1-Blue was used for transformations.

XL1-Blue: recA1, endA1, gyrA96, thi-1, hsdR17, supE44, relA1, lac[F'proAB, laclqZ $\triangle M 15, \operatorname{Tn} 10$ (Tetr)] (Stratagene)

\subsubsection{Antibiotics and Media}

For culture medium $32 \mathrm{~g}$ Luria Bertani (LB) was dissolved in 1 liter $\mathrm{dH}_{2} \mathrm{O}$ and autoclaved for $20 \mathrm{~min}$ at $121^{\circ} \mathrm{C}$. After cooling down to $50^{\circ} \mathrm{C}$ the selective antibiotic was added. The agar plates were poured under a sterile bench and stored at $4^{\circ} \mathrm{C}$ in the dark. As selective antibiotic Ampicillin was used (stock: 100 $\mathrm{mg} / \mathrm{ml}$ in $\mathrm{dH}_{2} \mathrm{O}$, working: $100 \mu \mathrm{g} / \mathrm{ml}$, stored in the dark at $-20^{\circ} \mathrm{C}$ ).

\subsubsection{Oligonucleotides}

Oligonucleotides were purchased from Sigma-Aldrich and dissolved in $\mathrm{HPLC}-\mathrm{H}_{2} \mathrm{O}$ to a concentration of $100 \mu \mathrm{M}$ or $500 \mu \mathrm{M}$. The following sequences of the oligonucleotides are written in 5' to 3' direction. 
2.1.4.1 RT-PCR Oligonucleotides (Primer)

\begin{tabular}{|c|c|c|c|}
\hline \multirow{2}{*}{ Gene } & \multicolumn{2}{|c|}{ Sequence } & \multirow{2}{*}{$\begin{array}{l}\mathrm{T}_{\mathrm{A}} \\
{ }^{\circ} \mathrm{C}\end{array}$} \\
\hline & Forward & reverse & \\
\hline neurog2 & GCGCGTTAAAGCTAACAACC & GTTCAGGTGGAGCTCAGAGG & 60 \\
\hline$t u b b 2 b$ & ACACGGCATTGATCCTACAG & AGCTCCTTCGGTGTAATGAC & 57 \\
\hline$t \mid x 3$ & GCCAACAAGTACAAGTGCACAG & CAGGAGCCAGACTCACATTGAC & 57 \\
\hline $\begin{array}{l}\text { prdm14 } \\
\text { a ex1-2 }\end{array}$ & CAGGACAAGTCACCAGGGAG & CAACTGCAACGAGTCCCTG & 60 \\
\hline $\begin{array}{l}\text { prdm14 } \\
b \text { ex1-2 }\end{array}$ & CTATGGTAGCGACTCAAGGACCG & CAACTGCAACGAGTCCCTG & 60 \\
\hline odc & GCCATTGTGAAGACTCTCTCCATTC & TTCGGGTGATTCCTTGCCAC & 56 \\
\hline
\end{tabular}

Table 2.1 Summary of RT-PCR oligonucleotides and their annealing temperatures

\subsubsection{Sequencing oligonucleotides}

\begin{tabular}{|l|l|c|}
\hline Primer & \multicolumn{1}{|c|}{ Sequence } & $\mathbf{T}_{\mathbf{A}}{ }^{\circ} \mathbf{C}$ \\
\hline SP6 & TTAGGTGACACTATAGAATAC & 56 \\
\hline T7 & TAATACGACTCACTATAGGGCGA & 56 \\
\hline T7 (pCS2+) & TCTACGTAATACGACTCACTATAG & 56 \\
\hline T3 & AATTAACCCTCACTAAAGGG & 56 \\
\hline GR7 & ATCCTGCATATAACAACTTC & 56 \\
\hline
\end{tabular}

Table 2.2 Summary of sequencing oligonucleotides and their annealing temperatures

\subsubsection{Morpholino oligonucleotides}

The morpholino oligonucleotides $(\mathrm{MO})$ used in this study were purchased from Gene Tools (Philomath, USA). The MOs were dissolved in RNase free $\mathrm{H}_{2} \mathrm{O}$ to a concentration of $20 \mathrm{ng} / \mathrm{nl}$ and stored at $4^{\circ} \mathrm{C}$. Before injection the $\mathrm{MO}$ were heated for $5 \mathrm{~min}$ at $65^{\circ} \mathrm{C}$. The following sequences of the $\mathrm{MO}$ are depicted in 5' to 3' direction.

\begin{tabular}{|l|c|}
\hline $\begin{array}{c}\text { Morpholino } \\
\text { Oligonucleotide }\end{array}$ & Sequence \\
\hline Prdm14a SpMO & GTGTATAACATTTACCTGTAGAACT \\
\hline Prdm14b SpMO & GTGTAACATTTACCTGTAGAAGTGC \\
\hline Standard control MO & CCTCTTACCTCAGTTACAATTTATA \\
\hline
\end{tabular}

Table 2.3 Summary of morpholino oligonucleotides 


\subsubsection{Overexpression constructs}

The following overexpression constructs used in this study have been described previously: NICD-pCS2 (Coffman et al., 1993), MT-Neurog2-pCS2+ (Ma et al., 1996), MT-GFP-pCS2+ (Rubenstein et al., 1997), MT-Wnt5a-pCS2+ (Damianitsch et al., 2009), Wnt8a-MT-pSP64T (Christian and Moon, 1993), ATF2-luc-pGL (van der Sanden et al., 2004), TOPFlash-luc-pGL3 (Korinek et al., 1997), Renilla-pRL-TK (Promega)

Prdm14-pCS2+: This construct harbors the full open reading frame of $X$. laevis prdm14. The open reading frame was PCR amplified using following primers: Prdm14_for_EcoR1: 5'-CGG AAT TCG ATG GCT CTG TCT GT-3' and Prdm14_rev_Xho1: 5'-GGC TCG AGA TAG GAG GCT TGA AT-3' with Prdm14-pCS2p+ (National Institute for Basis Biology, Japan; clone XL280n24ex) as a template. Prdm14 was subcloned into the pCS2+ expression vector using the EcoRI and Xhol restriction sites. For the transcription of sense mRNA, the construct was linearized with Notl and transcribed with the SP6 polymerase.

Prdm14-HA-pCS2+: This construct harbors the open reading frame of $X$. laevis prdm14 without the stop codon and an HA tag at the C-terminus. The open reading frame was $\mathrm{PCR}$ amplified using following primers: P14-HA_EcoRI_ START: 5'-GAG AAT TCA TGG CTC TGT CTG TT-3' and P14HA_Xhol_noSTOP2: 5'-TAC TCG AGG AGG CTG GAG TG-3' with Prdm14pCS2p+ (National Institute for Basis Biology, Japan; clone XL280n24ex) as a template. Prdm14 was subcloned into the pCS2+ HA vector (Damianitsch, 2009) using the EcoRI and Xhol restriction sites. This vector contains the human influenza hemaglutinin (HA) tag which allows the expression of HAtagged proteins. For the transcription of sense mRNA, the construct was linearized with Notl and transcribed with the SP6 polymerase.

Prdm14-GR-pCS2+: This construct harbors the open reading frame of $X$. laevis prdm14 without the stop codon. The prdm14 open reading frame was excised from prdm14-pCS2+HA and ligated into GRpCS2+ using the EcoRI and Xhol restriction sites. This vector contains the human glucocorticoid ligand-binding 
domain, which allows the induction of the translated protein by dexamethasone (Kolm and Sive, 1995). For the transcription of sense mRNA the construct was linearized with Notl and transcribed with the SP6 polymerase.

Prdm14-GR-HA-pCS2+: This construct harbors the open reading frame of $X$. laevis prdm14 without the stop codon. This vector contains the human glucocorticoid ligand-binding domain, which allows the induction of the translated protein by dexamethasone (Kolm and Sive, 1995). The GR-domain was PCR amplified using following primers: GR-tag_Xhol_for: ATC TCG AGA CCT CTG AAA ATC CT and GR-tag_+2_Xbal_noSTOP_rev: CGT CTA GAC ACT TTT GAT GAA ACA G using Prdm14-pCS2+GR as a template. The PCR product was cloned between the Xhol and Xbal sites of Prdm14-HA-pCS2+. For the transcription of sense mRNA the construct was linearized with Notl and transcribed with the SP6 polymerase.

\subsection{6 antisense-RNA-constructs}

\begin{tabular}{|c|c|c|c|c|}
\hline Marker & Vector & RE & $\begin{array}{c}\text { Poly- } \\
\text { merase }\end{array}$ & Reference \\
\hline$n$-tubulin/tubb2b & pBst KS & BamHI & T3 & Chitnis et al., 1995 \\
\hline sox2 & pBst SK & EcoRI & T7 & Mizuseki et al., 1998a \\
\hline sox10 & pCS2+ & Clal & T7 & Aoki et al., 2003 \\
\hline hox11L2/t/x3 & BstEll & Stul & T3 & Patterson \& Krieg, 1999 \\
\hline neurog1 & $\mathrm{pCS} 2 \mathrm{p}+$ & EcoRI & T7 & Nieber et al., 2009 \\
\hline neurog2 & pBst II & BamH1 & T3 & Ma et al., 1996 \\
\hline prdm14 & $\mathrm{pCS} 2 \mathrm{p}+$ & EcoRI & T7 & Klisch, 2006 \\
\hline pax3 & $\begin{array}{l}\text { pCMV- } \\
\text { Sport6 }\end{array}$ & Sall & T7 & Bang et al., 1999 \\
\hline wnt8 & pBst KS & BamHI & T3 & - \\
\hline zic1 & pCS2p+ & HindIII & T7 & Mizuseki et al., 1998a \\
\hline zic2 & pCS2p+ & EcoRI & T7 & Brewster et al., 1998 \\
\hline zic3 & pCS2p+ & EcoRI & T7 & Nakata et al., 1997 \\
\hline vglut & pBst KS & Notl & T3 & Gleason et al., 2003 \\
\hline foxd3 & pBst & EcoRI & T7 & Pohl and Knöchel, 2001 \\
\hline$h b 9 / m n x 1$ & pBst KS & Xbal & T3 & Saha et al., 1997 \\
\hline
\end{tabular}

Table 2.4 Summary of antisense RNA constructs 


\subsection{Methods}

\subsubsection{DNA-standard methods}

\subsubsection{Polymerase chain reaction (PCR)}

\subsection{Reverse transcription-PCR (RT-PCR)}

For semi-quantitative RT-PCR $2.5 \mu \mathrm{l}$ cDNA was used in a $12.5 \mu \mathrm{l}$ reaction containing 1x Go Taq green reaction buffer (Promega), $0.2 \mathrm{mM}$ of each primer and 0.5 units GoTaq DNA-polymerase (Promega). The amplification was carried out in a thermocycler (Biometra) with following conditions: 2 min $95^{\circ} \mathrm{C}$, $45 \sec 95^{\circ} \mathrm{C}, 30 \mathrm{sec} \mathrm{T}_{\mathrm{A}}$ of primers $-2^{\circ} \mathrm{C}, 30 \mathrm{sec} 72^{\circ} \mathrm{C}, 5 \mathrm{~min} 72^{\circ} \mathrm{C}$. The number of cycles between step 2 and step 4 varied between the primer pairs and ranged between 25-35 cycles. The RT-PCR reaction were analyzed on a $2 \%$ agarose gel and documented in a Chemidoc chamber (Biorad) mounted with an Intas camera.

\subsubsection{PCR Cloning}

For molecular cloning the High Fidelity Enzyme Mix (Fermentas) was used. A PCR reaction contained 1x High Fidelity Buffer with $15 \mathrm{mM} \mathrm{MgCl}, 0.2$ $\mathrm{mM}$ dNTP mix (Thermo Scientific), $0.75 \mu \mathrm{M}$ of each primer, $0.1 \mathrm{ng} / \mu \mathrm{l}$ template DNA and $0.1 \mathrm{U} / \mu \mathrm{l}$ High Fidelity PCR enzyme mix. The amplification was carried out in a thermocycler (Biometra) with following conditions: 2 min $95^{\circ} \mathrm{C}, 45 \mathrm{sec}$ $95^{\circ} \mathrm{C}, 45 \mathrm{sec} \mathrm{T}_{\mathrm{A}}$ of primers $-2{ }^{\circ} \mathrm{C}, 1 \mathrm{~min} / \mathrm{kb} 72^{\circ} \mathrm{C}, 5 \mathrm{~min} 72^{\circ} \mathrm{C}$. The number of cycles between step 2 and step 4 varied between the primer pairs and ranged between 30-40 cycles.

\subsubsection{Agarose gel electrophoresis}

TAE (Tris-acetate-EDTA): 40 mM Tris-acetate, 2 mM EDTA, pH 8.5

Restriction- and PCR-fragments were analyzed by agarose gel electrophoresis (Sharp et al., 1973). Depending on the fragment size the percentage of the agarose gel was adapted (0.7-2\%). Agarose powder was heated and dissolved in $1 x$ TAE buffer. To visualize the DNA fragments ethidium bromide $(0.5 \mu \mathrm{g} / \mathrm{ml})$ was added to the liquid agarose gel. The 
electrophoresis was performed in a horizontal electrophoresis chamber filled with $1 \mathrm{x}$ TAE at $100 \mathrm{~V}$. For determination of the fragment sizes, standard DNA ladders (Fast Ruler DNA Ladder, Fermentas) were used. The documentation of the gel was carried out in a ChemiDoc (Bio-Rad) documentation chamber using an Intas camera and software.

\subsubsection{Gel purification of PCR and restriction fragments}

For isolation of DNA fragments from agarose gels, the agarose gel containing the desired fragment was excised and the DNA purified using the Fragment CleanUp Kit (Invitek) according to the manufacturer's instructions.

\subsubsection{DNA restriction digestion}

For restriction digestions of DNA restriction endonucleases were used from Fermentas Life Sciences according to manufacturer's instructions.

\subsubsection{Ligation}

For standard ligation reactions T4 DNA ligase (10 U/ $/$ ) (Fermentas Life Science) was used according to manufacturer's protocol. The ligation reaction was incubated overnight at $16^{\circ} \mathrm{C}$.

\subsubsection{Chemical transformation of bacterial cells}

For chemical transformations the chemically competent Escherichia colistrain XL1-Blue was used. Ligation or PCR reactions were added to 100-200 $\mu$ l thawed cells and incubated on ice for $30 \mathrm{~min}$. Afterwards the cells were heat shocked for $60-90 \mathrm{sec}$ at $42^{\circ} \mathrm{C}$ and immediately cooled for $3 \mathrm{~min}$ on ice. LB medium $(800 \mu \mathrm{l})$ was added to the cells which were then incubated for 30-45 min under constant shaking (300 rpm) at $37^{\circ} \mathrm{C}$. After the incubation, the cells were centrifuged (30 sec, 10,000 rpm) and $800 \mu$ of the overlying medium was discarded. The cells were resuspended in the remaining medium and seeded on LB agar plates containing selective antibiotic. The LB plates were incubated overnight at $37^{\circ} \mathrm{C}$. 


\subsubsection{Plasmid preparation}

The GeneJET Plasmid Miniprep kit (Fermentas) was used according to manufacturer's instructions to isolate DNA. For the isolation of DNA in preparative amounts $(1 \mu \mathrm{g} / \mu \mathrm{l})$ the NucleoBond Xtra Midi kit (Macherey-Nagel) was used following the manufacturer's protocol.

For DNA quantification the NanoDrop 2000c spectrophotometer (Thermo Scientific) was used.

\subsubsection{DNA sequencing}

For DNA sequencing, the chain termination method was used (Sanger et al., 1977). The sequencing was performed using the Big Dye Terminator Cycle Sequencing Kit (Applied Biosystems) according to manufacturer's instructions. The sequencing PCR mixture contained 200-400 ng DNA, $2 \mu \mathrm{l}$ seq mix, $2 \mu \mathrm{l}$ seq buffer and 1 pmol sequencing primer in a total of $10 \mu$ reaction volume. The reaction was performed in a thermocycler using following program: 2 min $95^{\circ} \mathrm{C}$, $30 \sec 95^{\circ} \mathrm{C}, 30 \sec 55^{\circ} \mathrm{C}, 4 \min 72^{\circ} \mathrm{C}$, with 26 cycles between step 2 and 4 . For purification of the sequencing reaction, $1 \mu \mathrm{NaAc}$ (3 M), $1 \mu$ I EDTA (125 $\mathrm{mM}$ ) and $50 \mu \mathrm{l}$ 100\% ethanol were added to the reaction mixture and incubated for $5 \mathrm{~min}$ at RT. After centrifugation for $15 \mathrm{~min}$ at 13,000 rpm, the DNA pellet was washed with $70 \%$ ethanol, air-dried and dissolved in $15 \mu \mathrm{H}$ HiDi Buffer (Applied Biosystems). The automated sequencing was performed in the $3130 \mathrm{xl}$ Genetic Analyzer (Applied Biosystems).

\subsubsection{RNA standard methods}

\subsubsection{In vitro synthesis of capped sense mRNA}

Capped sense mRNA was synthesized using the SP6 or T7 mMessage mMachine Kits (Ambion) according to manufacturer's protocol. A reaction of 20 $\mu \mathrm{l}$ contained $1 \mu \mathrm{g}$ of linearized plasmid and was incubated at $37^{\circ} \mathrm{C}$ for 2 hours. After DNase digestion using $5 \mathrm{U}$ of Turbo DNase I, the synthesized mRNA was purified using the Illustra RNAspin Mini kit (GE Healthcare) and eluted in $30 \mu \mathrm{l}$ RNase free water at $80^{\circ} \mathrm{C}$. RNA concentration was measured using the NanoDrop 2000c spectrophotometer (Thermo Scientific) and the quality was 
checked on a 1\% agarose gel. The RNA was aliquoted (2-4 $\mu \mathrm{l})$ and stored at $80^{\circ} \mathrm{C}$.

\subsubsection{In vitro synthesis of antisense RNA}

For the detection of in vivo transcripts antisense RNA was used in whole mount in situ hybridizations (WMISH).

A standard reaction of $25 \mu \mathrm{l}$ contained 1 ng linearized plasmid, $1 x$ Transcriptionbuffer (Fermentas), $1 \mathrm{mM}$ rATP, rGTP, rCTP (Boehringer), 0.64 mM rUTP (Boehringer), $0.36 \mathrm{mM}$ Digoxygenin-rUTP (Boehringer), $0.03 \mu \mathrm{M}$ DTT, $1.6 \mathrm{U} / \mu \mathrm{l}$ Ribolock RNase inhibitor (Fermentas), $1.2 \mathrm{U} / \mu \mathrm{l} \mathrm{T3}$, T7 or SP6 RNA-Polymerase, add RNase-free water. The reaction was incubated for three hours at $37^{\circ} \mathrm{C}$, followed by template digestion with $0.2 \mathrm{U} / \mu \mathrm{l}$ Turbo DNasel (Ambion) for $30 \mathrm{~min}$ at $37^{\circ} \mathrm{C}$. The RNeasy Mini kit (Qiagen) was used to purify the synthesized antisense RNA according to manufacturer's instructions. The RNA was eluted twice with $50 \mu \mathrm{l}$ RNase-free water at $80^{\circ} \mathrm{C}$. The quality was checked on a $1 \%$ agarose gel. The antisense RNA was stored at $-20^{\circ} \mathrm{C}$ in $1 \mathrm{ml}$ hybridization mix (see whole mount in situ hybridization).

\subsubsection{Total RNA isolation from ectodermal explants and whole embryos}

For the isolation of total RNA three embryos or 50-100 ectodermal explants were fixed in liquid nitrogen and lysated in $400 \mu l$ peqGOLD TriFast reagent (Peqlab) using a 29-gauge syringe and afterwards vortexed for $30 \mathrm{sec}$. After addition of $80 \mu \mathrm{l}$ chloroform (Roth) and vortexing for $30 \mathrm{sec}$, the samples were centrifuged for $10 \mathrm{~min}$ at $13,000 \mathrm{rpm}$ and $4^{\circ} \mathrm{C}$. The supernatant $(200 \mu \mathrm{l})$ was transferred into a new eppendorf tube. $200 \mu$ chloroform were added and the sample vortexed for $30 \mathrm{sec}$ and centrifuged for $5 \mathrm{~min}$ at $13,000 \mathrm{rpm}$ at $4^{\circ} \mathrm{C}$. The supernatant $(180 \mu \mathrm{l})$ was transferred into a new eppendorf tube and $180 \mu \mathrm{l}$ isopropanol were added. After vortexing, the samples were kept overnight at $20^{\circ} \mathrm{C}$ for precipitation. After precipitation the samples were centrifuged for 30 min at $13,000 \mathrm{rpm}$ at $4^{\circ} \mathrm{C}$ and the pellet was washed with $400 \mu \mathrm{l} 70 \%$ ethanol. After air drying the pellet was dissolved in $12.5 \mu \mathrm{l}$ RNase-free water and DNase

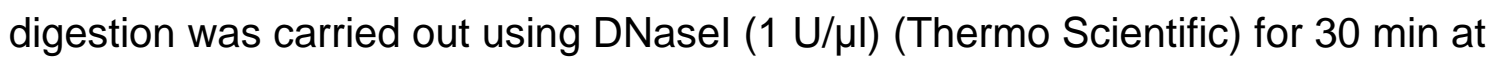
$37^{\circ} \mathrm{C}$. The DNasel was denatured by incubation at $70^{\circ} \mathrm{C}$ for 10 minutes and the 
RNA concentration measured on the NanoDrop 2000c spectrophotometer (Thermo Scientific). The quality of the RNA was analyzed with the 2100 Bioanalyzer (Agilent). To check for genomic DNA contamination, a control PCR with $\mathrm{H} 4$ or ODC was performed.

\subsubsection{Reverse transcription}

For the synthesis of $10 \mu \mathrm{l}$ cDNA, 50-75 ng RNA was used in a standard reaction mix containing $1 \times$ Go Taq flexi buffer, $5 \mathrm{mM} \mathrm{MgCl}$ (Fermentas), 2.5 mM random hexamer (Invitrogen), $1 \mathrm{mM}$ dNTP mix (Thermo Scientific), $0.8 \mathrm{U} / \mu \mathrm{l}$ Ribolock RNase inhibitor (Thermo Scientific), $2 \mathrm{U} / \mu \mathrm{l}$ MulV reverse transcriptase (Roche). The reaction was carried out in a thermocycler. After an initial incubation for $20 \mathrm{~min}$ at $20^{\circ} \mathrm{C}$ the reaction was incubated for 1 hour at $42^{\circ} \mathrm{C}$ and terminated at $95^{\circ} \mathrm{C}$ for $5 \mathrm{~min}$.

\subsubsection{RNA-sequencing}

\subsection{Total RNA isolation from ectodermal explants}

For the isolation of total RNA for RNA-sequencing 70-100 ectodermal explants were fixed in liquid nitrogen and lysates prepared in $360 \mu$ peqGOLD TriFast reagent (Peqlab) using a 29-gauge syringe and afterwards incubated for $10 \mathrm{~min}$ at RT. After addition of $72 \mu$ chloroform the solution was incubated at RT for $5 \mathrm{~min}$ and centrifuged for $20 \mathrm{~min}$ with $13,000 \mathrm{rpm}$ at $4^{\circ} \mathrm{C}$. The upper phase was transferred into a new Eppendorf tube to which $200 \mu$ l chloroform was added. The solution was centrifuged for $10 \mathrm{~min}$ at $4^{\circ} \mathrm{C}$ and the upper phase transferred into a new Eppendorf tube. After adding $180 \mu$ isopropanol the RNA was precipitated overnight at $-20^{\circ} \mathrm{C}$. The next day, the solution was centrifuged for $30 \mathrm{~min}$ at $4^{\circ} \mathrm{C}$. The pellet was washed with $70 \%$ ethanol and centrifuged for $5 \mathrm{~min}$ at $4^{\circ} \mathrm{C}$. The pellet was air dried, and resuspended in $43.5 \mu \mathrm{l}$ Rase-free water. For the digestion of genomic DNA, the RNA was incubated for $1 \mathrm{~h}$ at $37^{\circ} \mathrm{C}$ in a $50 \mu \mathrm{l}$ reaction containing $1 \mathrm{x}$ DNasel reaction buffer (Thermo Scientific), $1 \mu$ DNasel (Thermo Scientific) and $0.5 \mu$ l RNase inhibitor (Thermo Scientific). To stop the DNase treatment, $150 \mu$ l RNase free water and $200 \mu \mathrm{l}$ phenol/chloroform/isoamylalcohol (25/24/1, Roth) was added to the RNA. After centrifugation for $10 \mathrm{~min}$ at $4^{\circ} \mathrm{C}$ the upper phase was transferred to a new 
Eppendorf tube, 1 vol. of chloroform/isoamylalcohol (24/1) was added. Afterwards the RNA was centrifuged for $10 \mathrm{~min}$ at $4^{\circ} \mathrm{C}$. The supernatant was transferred to a new Eppendorf tube to which 1/10 vol. of $5 \mathrm{M}$ ammonium acetate and 1 vol. of isopropanol was added. The solution was incubated overnight at $-20^{\circ} \mathrm{C}$. The following day the RNA was centrifuged for $30 \mathrm{~min}$ at $4^{\circ} \mathrm{C}$ and the pellet washed twice with $70 \%$ ethanol and centrifuged for 5 min at $4^{\circ} \mathrm{C}$. The pellet was air dried and resuspended in $20 \mu \mathrm{l}$ Nase free water. The quality of the RNA was analyzed with the 2100 Bioanalyzer (Agilent). To check for genomic DNA contamination, a control PCR with H4 or ODC was performed.

\subsection{Sample preparation and sequencing}

LIbrary preparation and sequencing was performed by the DNA Microarray and Deep-Sequencing Facility Göttingen (UMG) Transkriptomanalyselabor (TAL). Before sequencing, the RNA-libraries were prepared with the "TruSeq RNA Sample Prep Kit v2" (Illumina) following the manufacturer's protocol. The single read (50 bp) sequencing was performed using the HiSeq 2000 (Illumina). Three independent biological replicates were used for each sequencing reaction. The quality of the sequencing was controlled using the FastQ software (http://www.bioinformatics.babraham.ac.uk/projects/fastqc/).

\subsection{Sequencing alignment (performed by TAL)}

The sequence reads were aligned to the genome reference sequence of $X$. tropicalis (Joint Genome Institute assembly v4.2) using the STAR alignment software version 2.3.0e (Dobin et al., 2012). For the alignment, 5 mismatches were allowed within 50 bases.

\subsection{Statistical analysis (performed by TAL)}

For normalization and identification of differentially expressed genes the R/Bioconductor environment (www.bioconductor.org) with the DESeq2 package version 1.2.10 (Anders and Huber, 2010) was used. Genes showing at least a 2-fold change and a FDR-corrected p-value $<0.05$ were considered as candidate genes. 


\subsubsection{X. laevis embryo culture and micromanipulations}

For detailed descriptions refer to Sive et al., 2010.

10x MBS: $880 \mathrm{mM} \mathrm{NaCl}, 10 \mathrm{mM} \mathrm{KCl}, 10 \mathrm{mM} \mathrm{MgSO}_{4}, 25 \mathrm{mM} \mathrm{NaHCO}$, $50 \mathrm{mM}$ HEPES, $\mathrm{pH} 7.8$

1x MBS: 1x MBS, $0.7 \mathrm{mM} \mathrm{CaCl}_{2}$

5x MBS AC: $880 \mathrm{mM} \mathrm{NaCl}, 10 \mathrm{mM} \mathrm{KCl}, 10 \mathrm{mM} \mathrm{MgSO}_{4}, 25 \mathrm{mM} \mathrm{NaHCO}_{3}, 50$ mM HEPES, $2.1 \mathrm{mM} \mathrm{CaCl}_{2}, 1.7 \mathrm{mM} \mathrm{Ca}\left(\mathrm{NO}_{3}\right), \mathrm{pH} 7.8$

Dejelly solution: $2 \%(w / v)$ L-cysteine hydrochloride in $0.1 \times \mathrm{MBS}, \mathrm{pH} 8$ Injection buffer: $1 \%$ ficoll in $1 \times$ MBS

Nile Blue staining solution: 0.01 (w/v) nile blue chloride, $89.6 \mathrm{mM} \mathrm{Na}_{2} \mathrm{HPO}_{4}$, $10.4 \mathrm{mM} \mathrm{NaH}_{2} \mathrm{PO}_{4}, \mathrm{pH} 7.8$

Agarose dishes: $60 \mathrm{~mm}$ petri-dishes coated with 1\% agarose made with $0.8 \mathrm{x}$ MBS AC

\subsubsection{Stimulation of eggs}

To induce the $X$. laevis female frogs to lay eggs, $1000 \mathrm{U}$ of human chorionic gonadotropin (HCG, Sigma Aldrich) was injected subcutaneous into the dorsal lymph sac the evening before desired egg laying. The frogs were kept at $16^{\circ} \mathrm{C}$ and started egg-laying $12-24$ hours after HCG injection.

\subsubsection{Preparation of $X$. laevis testis}

For in vitro fertilization of the laid eggs, a male $X$. laevis was sacrificed by submersion in $0.05 \%$ benzocaine for $30 \mathrm{~min}$ at RT. The testis was removed and stored at $4^{\circ} \mathrm{C}$ in $1 \times$ MBS for approximately one week.

\subsubsection{In vitro fertilization}

Egg-laying was stimulated by massaging the abdomen and sides of the frogs. The layed eggs were fertilized by addition of $1 / 4$ Vol. macerated testis in $1 x$ MBS which was diluted with $3 / 4$ Vol. of $\mathrm{H}_{2} \mathrm{O}$. To prevent dehydration the fertilized eggs were covered in 1x MBS. 


\subsubsection{Microinjections}

The jelly coat of the embryos was removed by treatment with dejelly solution and washing 3-4 times with $0.1 \times$ MBS. The microinjections were performed in injection buffer on a cooling plate set to $12.5^{\circ} \mathrm{C}$ using a borosilicate glass capillary (Harvard Apparatus), which was pulled with a needle puller (PN30, Scientific Products $\mathrm{GmbH}$ ). The needles were back-loaded with the injection solution using microloaders (Eppendorf) and placed into a mechanical micromanipulator (M1, H. Sauer Laborbedarf) that was attached to a microinjector (PV 820, H. Sauer Laborbedarf). Depending on the experimental setup, $4 \mathrm{nl}$ of the injection solution was injected animally into either one or two blastomeres of two-cell stage embryos, or into one dorsal blastomere of a fourcell stage embryos. After injection, the embryos were kept for at least one hour in injection buffer and were then transferred into 0.1x MBS and cultured at different temperatures $\left(12.5-18^{\circ} \mathrm{C}\right)$ until the desired developmental stages were reached. The developmental stages were determined according to Nieuwkoop and Faber (1967).

\subsubsection{X. laevis ectodermal explants ("animal caps")}

Ectodermal explants were excised at blastula stage (stage 8-9) in $0.8 x$ MBS on an agarose coated petri-dishes on a cooling plate set at $12.5^{\circ} \mathrm{C}$. The vitelline membrane was removed with forceps and the ectodermal explant was excised from the animal hemisphere using a Gastromaster (Xenotek Engineering, Bellville, USA) set to "yellow high" with a yellow tip. The explants were cultivated in 0.8x MBS until control sibling embryos reached the desired stage. Explants were snap frozen in liquid nitrogen and stored at $-80^{\circ} \mathrm{C}$ until RNA isolation.

\subsubsection{Dexamethasone treatment}

To control the time point of protein activity, the coding sequence of the gene of interest was fused to the ligand binding domain of the human glucocorticoid receptor (Kolm and Sive, 1995). To induce protein activity, the fusion construct injected embryos or animal caps were cultivated in $0.1 \mathrm{x}$ or $0.8 \mathrm{x}$ 
MBS containing $10 \mu \mathrm{M}$ dexamethasone in the dark until the desired stage was reached.

\subsubsection{7 $\beta$-Gal staining}

10x MEM: 1 M Mops, 20 mM EGTA, $10 \mathrm{mM} \mathrm{MgSO}_{4}$, pH 7.4 sterile filtered and stored in the dark

10x PBS: $1.8 \mathrm{M} \mathrm{NaCl}, 1 \mathrm{M} \mathrm{KCl}, 65 \mathrm{mM} \mathrm{Na}_{2} \mathrm{HPO}_{4}, 18 \mathrm{mM} \mathrm{KH}_{2} \mathrm{PO}_{4}, \mathrm{pH} 7.4$

Dent's solution: $20 \%(\mathrm{v} / \mathrm{v})$ DMSO in methanol

$\mathrm{K}_{3} \mathrm{Fe}(\mathrm{CN})_{6}: 0.5 \mathrm{M}$ in $\mathrm{H}_{2} \mathrm{O}$, stored in the dark

$\mathrm{K}_{4} \mathrm{Fe}(\mathrm{CN})_{6}: 0.5 \mathrm{M}$ in $\mathrm{H}_{2} \mathrm{O}$, stored in the dark

MEMFA: 4\% (v/v) formaldehyde (37\%) in 1x MEM

X-Gal: 40 mg/ml 5-bromo-4-chloro-3-indolyl-s-D-galactopyranoside in formamide, stored at $-20^{\circ} \mathrm{C}$ in the dark.

X-Gal staining solution: $1 \mathrm{mg} / \mathrm{ml} \mathrm{X-Gal,} 5 \mathrm{mM} \mathrm{K}_{3} \mathrm{Fe}(\mathrm{CN})_{6}, 5 \mathrm{mM} \mathrm{K}_{4} \mathrm{Fe}(\mathrm{CN})_{6}, 2$ $\mathrm{mM} \mathrm{MgCl} 2$ in $1 x$ PBS

To distinguish the uninjected from the injected side of an embryo lacz mRNA ( $\beta$-Gal) was co-injected as a lineage tracer (Hardcastle et al., 2000). The embryos were fixed at the desired stages for 20 min in MEMFA, washed three times for (10 min each) in 1X PBS and transferred to X-Gal staining solution. Staining took place in the dark until sufficient staining was observed (20-30 $\min )$. The reaction was stopped by washing three times (10 min each) with $1 x$ PBS followed by fixation for $25 \mathrm{~min}$ in MEMFA. Finally, the embryos were dehydrated by washing several times in absolute ethanol and stored at $-20^{\circ} \mathrm{C}$. For pH3 staining and fluorescent immunostaining, the embryos were dehydrated in absolute methanol, fixed in Dent's fix for at least 24 hours at $20^{\circ} \mathrm{C}$ and stored in absolute methanol at $-20^{\circ} \mathrm{C}$.

\subsubsection{Whole mount in situ-hybridization (WMISH)}

10X PBS: $1.8 \mathrm{M} \mathrm{NaCl}, 1 \mathrm{M} \mathrm{KCl,} 65 \mathrm{mM} \mathrm{Na}_{2} \mathrm{HPO}_{4}, 18 \mathrm{mM} \mathrm{KH}_{2} \mathrm{PO}_{4}, \mathrm{pH} 7.4$ 20X SSC: $3 \mathrm{M} \mathrm{NaCl}, 0.3 \mathrm{M}$ NaCitrate, $\mathrm{pH} 7.2$ - 7.4

5X MAB: 500 mM maleic acid, 750 mM NaCl, pH 7.5 
Antibody solution: 2\% Boehringer Mannheim Blocking reagent (BMB), 20\% heat treated horse serum (Gibco), 1:2000 dilution of anti-digoxigenin or antifluorescein antibody coupled to alkaline phosphatase (Roche) in 1X MAB

APB: $100 \mathrm{mM}$ Tris- $\mathrm{HCl}, \mathrm{pH}$ 9.0, $50 \mathrm{mM} \mathrm{MgCl}_{2}, 100 \mathrm{mM} \mathrm{NaCl}$,

$0.1 \%$ TWEEN-20

BCIP: $50 \mathrm{mg} / \mathrm{mL}$ in $100 \%$ dimethylformamide; stored at $-20^{\circ} \mathrm{C}$

Bleaching solution: $50 \%$ formamide, $1-2 \% \mathrm{H}_{2} \mathrm{O}_{2}$ in $5 \times \mathrm{SSC}$

Color reaction solution: $80 \mu \mathrm{g} / \mathrm{ml} \mathrm{NBT}, 175 \mu \mathrm{g} / \mathrm{ml}$ BCIP in APB

EtOH series: $100 \%, 75 \%, 50 \%$ ethanol in $\mathrm{H}_{2} \mathrm{O}, 25 \%$ ethanol in PTw

Hybridization Mix (Hyb Mix): 50\% formamide, $1 \mathrm{mg} / \mathrm{ml}$, Torula-RNA, $10 \mu \mathrm{g} / \mathrm{ml}$ Heparin, 1X Denhardt's, 0.1\% Tween-20, 0.1\% CHAPS, 10 mM EDTA in 5X SSC

MeOH series: $100 \%, 75 \%, 50 \%, 25 \%$ methanol in $\mathrm{H}_{2} \mathrm{O}$

MAB/BMB: $2 \% B M B$ in $1 X M A B$

MAB/BMB/HS: 2\% BMB, 20\% heat-treated horse serum in 1X MAB

NBT: $100 \mathrm{mg} / \mathrm{mL}$ in $70 \%$ dimethylformamide; stored at $-20^{\circ} \mathrm{C}$

PTw: 0.1\% Tween-20 in 1X PBS

PTw/FA: 4\% (v/v) formaldehyde in PTw

Proteinase $\mathrm{K}$ : $5 \mu \mathrm{g} / \mathrm{ml}$ proteinase $\mathrm{K}$ (Merck) in 0.1X PBS

RNAse Solution: $10 \mu \mathrm{g} / \mathrm{ml}$ RNAse A, $0.01 \mathrm{U} / \mathrm{ml}$ RNAse T1 in 2X SSC

Triethanolamine: 0.93 triethanolamine in $\mathrm{H}_{2} \mathrm{O}, \mathrm{pH} 7.5$

To visualize temporal and spatial gene expression, WMISH was performed as previously described (Harland, 1991; Hollemann and Pieler, 1999) using digoxigenin-UTP or fluorescin-UTP labelled antisense RNA. All steps were performed with mild shaking at room temperature, if not otherwise noted.

The embryos were rehydrated in an ethanol series (75\%, 50\%, and $25 \%$ ) and washed four times for $5 \mathrm{~min}$ in PTw. To make the surface of the embryo penetrable for the antisense RNA probe, they were treated with PTw/proteinase $\mathrm{K}$ solution (10 $\mu \mathrm{g}$ proteinase $\mathrm{K} / \mathrm{ml} \mathrm{PTw})$. Stage 14 embryos were treated for 6 min while Stage 27-28 embryos were treated for 17 minutes. To stop the proteinase $\mathrm{K}$ treatment the embryos were washed twice for $5 \mathrm{~min}$ with triethanolamine. To prevent unspecific binding of the antisense RNA probe, free amino-acid ends were blocked by addition of $25 \mu$ lacetic anhydride to the 
triethanolamine. After incubation for $5 \mathrm{~min}$, an additional $25 \mu \mathrm{l}$ acetic anhydrite was added to the triethanolamine. Following acetylation, the embryos were washed two times for $5 \mathrm{~min}$ in PTw, refixed for $20 \mathrm{~min}$ in PTw/FA and finally washed 5 times for $5 \mathrm{~min}$ in PTw. For pre-hybridization, the embryos were incubated with $\mathrm{Hyb}$ Mix for $5 \mathrm{~h}$ at $65^{\circ} \mathrm{C}$. After pre-hybridization the embryos were incubated overnight at $65^{\circ} \mathrm{C}$ in $\mathrm{Hyb}$ Mix containing the antisense RNA probe.

The following day, the antisense RNA probe containing Hyb Mix was exchanged with fresh Hyb Mix for 10 min at $65^{\circ} \mathrm{C}$ followed by washing three times for $15 \mathrm{~min}$ each at $65^{\circ} \mathrm{C}$ with $2 \mathrm{x}$ SSC. To remove remaining nonhybridized antisense RNA probe, the embryos were treated with RNase solution at $37^{\circ} \mathrm{C}$ and afterwards washed once in $2 x$ SSC for 5 min at RT, twice in $0.2 x$ SSC at $65^{\circ} \mathrm{C}$ and two times for $15 \mathrm{~min}$ at RT in $1 \times$ MAB. To avoid unspecific binding of the antibody, the embryos were blocked for 15 min in MAB/BMB and afterwards for $40 \mathrm{~min}$ in $\mathrm{MAB} / \mathrm{BMB} / \mathrm{HS}$. The embryos where then incubated overnight at $4^{\circ} \mathrm{C}$ in the antibody solution.

The next day, the embryos were washed at least 5 times for $10 \mathrm{~min}$ in 1x $M A B$. For the color reaction, the embryos were first washed twice for $5 \mathrm{~min}$ at $4^{\circ} \mathrm{C}$ in fresh $\mathrm{APB}$ and then transferred to the color reaction solution. The reaction took place at $4^{\circ} \mathrm{C}$, in the dark, until staining was sufficient. To stop the color reaction and remove background staining, the embryos were dehydrated in $100 \%$ methanol until no background color leached out of the embryos. For rehydration the embryos were incubated in sequentially in 100\%, 75\%, 50\% and 25\% methanol and afterwards fixed in MEMFA for $15 \mathrm{~min}$. The embryos were stored indefinitely in MEMFA. To remove the pigmentation, the embryos were incubated in bleaching solution until pigmentation was gone and afterwards washed two times in 5x SSC followed by fixation and storage in MEMFA.

\subsubsection{Phosphorylated Histone 3 (pH3) staining}

10X PBS: $1.8 \mathrm{M} \mathrm{NaCl}, 1 \mathrm{M} \mathrm{KCl}, 65 \mathrm{mM} \mathrm{Na}_{2} \mathrm{HPO}_{4}, 18 \mathrm{mM} \mathrm{KH}_{2} \mathrm{PO}_{4}$, pH 7.4 $1^{\circ} \mathrm{AB}$ solution: $20 \%$ horse serum, 5\% DMSO, 1/270 dilution rabbit anti-pH3 antibody (Biomol)

$2^{\circ}$ AB solution: $20 \%$ horse serum, 5\% DMSO, 1/750 dilution goat anti-rabbit IgG horseradish peroxidase-coupled secondary antibody (Sigma) 
APB: $100 \mathrm{mM}$ Tris- $\mathrm{HCl}, \mathrm{pH}$ 9.0, $50 \mathrm{mM} \mathrm{MgCl}_{2}, 100 \mathrm{mM} \mathrm{NaCl}$, $0.1 \%$ tween-20

Color reaction solution: $80 \mu \mathrm{g} / \mathrm{ml} \mathrm{NBT,} 175 \mu \mathrm{g} / \mathrm{ml}$ BCIP in APB

MeOH series: $100 \%, 75 \%, 50 \%$, in $\mathrm{H} 2 \mathrm{O}$ and $25 \%$ methanol in 1 PPBS

PBS-TB: $0.05 \%$ Tween-20, 0.2\% BMB in $1 \times$ PBS

PBS-TBN: $0.3 \mathrm{M} \mathrm{NaCl}$ in PBS-TB

Proteinase K: $5 \mu \mathrm{g} / \mathrm{ml}$ Proteinase K (Merck) in 0.1X PBS

To mark mitotically active cells in whole embryos, phosphohistone H3 staining was performed as previously described (Dent et al., 1989). Embryos were fixed in Dent's fix after $\beta$-gal staining and rehydrated in a $\mathrm{MeOH}$ series to PBS. After Proteinase $\mathrm{K}$ treatment for $10 \mathrm{~min}$, the embryos were washed two times in PBS-TB for $10 \mathrm{~min}$ at RT. For the first antibody reaction, the embryos were pre-incubated in PBS with $20 \%$ horse serum. After $2 \mathrm{~h}$ at RT the embryos were transferred to the $1^{\circ} \mathrm{AB}$ solution and incubated overnight at $4^{\circ} \mathrm{C}$. The next day, the embryos were washed twice with PBS-TB for $2 \mathrm{~h}$ at RT and afterwards with PBS-TB overnight at $4^{\circ} \mathrm{C}$. The following day, the embryos were washed for $2 \mathrm{~h}$ with PBS-TBN, 5 min with PBS-TB and overnight at $4^{\circ} \mathrm{C}$ in $2^{\circ} \mathrm{AB}$ solution. The next day, the embryos were washed twice in PBS-TB for 30 min, once for 30 min in PBS-TBN, once in PBS-TB for $5 \mathrm{~min}$ and then washed overnight in PBS-TB at $4^{\circ} \mathrm{C}$. For the color reaction the embryos were first washed with PBSTB for $10 \mathrm{~min}$, twice in fresh APB for $5 \mathrm{~min}$ at $4^{\circ} \mathrm{C}$ and afterwards transferred to the color reaction solution. When the desired staining was reached, the embryos were dehydrated and stored in $100 \%$ methanol at $-20^{\circ} \mathrm{C}$.

\subsubsection{Fluorescent immunostaining}

\section{Blocking solution:}

Murray's clear: benzyl benzoate/benzyl alcohol, 2/1

TBS: 50mM Tris-HCl, $150 \mathrm{mM} \mathrm{NaCl}, \mathrm{pH} 7.6$

TBST: $0.05 \%$ Tween-20 in TBS

Fluorescent immunostaining was performed to detect acetylated tubulin, which marks the axons of neurons (Friedmann et al., 2012). After $\beta$-gal staining, embryos were fixed overnight in Dent's fix and dehydrated for at least $24 \mathrm{~h}$ in 
$100 \%$ methanol. For rehydration the embryos were subjected to a $\mathrm{MeOH}$ series to TBS. To prevent nonspecific antibody binding, the embryos were blocked for 30 min in blocking solution at RT. The primary antibody was added directly to the blocking solution (anti-acetylated tubulin, 1/200, Sigma-Aldrich) and incubated overnight at $4^{\circ} \mathrm{C}$. The following day, the embryos were washed at RT for $3 \mathrm{~h}$ in TBST that was exchanged every $30 \mathrm{~min}$. For the second antibody reaction, the embryos were blocked in blocking solution for 15 at RT. The secondary antibody (goat anti-mouse Alexa 488, 1/300, Invitrogen) was added directly to the blocking solution and incubated overnight at $4^{\circ} \mathrm{C}$. The next day, the embryos were washed at RT for $3 \mathrm{~h}$ in TBST that was exchanged every 30 min. The embryos were dehydrated in 100\% methanol and cleared in Murray's clear for confocal imaging on a LSM780 (Zeiss).

\subsubsection{Luciferase reporter assay}

For luciferase reporter assay two cell stage embryos were injected animally into both blastomeres with 100 pg Atf2-Firefly-luciferase reporter (van der Sanden et al., 2004) or 200 pg TOPFlash-Firefly-luciferase reporter (Fujimi et al., 2011) together with $10 \mathrm{pg}$ Renilla luciferase reporter (Promega) for normalization. The reporters were injected alone or co-injected with either 500 pg MT-wnt5b, 100 pg wnt8a-MT or 500 pg prdm14-GR respectively. At least six injected embryos at stage 14 were collected per sample and two samples were tested for one condition. For measurement of the Firefly and Renilla luciferase activity the Dual Luciferase Reporter Kit (Promega) and a Centro LB960 luminometer (Berthold Technologies) were used according to manufacturer's protocols.

\subsubsection{Vibratome sectioning}

Gelatin/Albumin: $4.9 \mathrm{mg} / \mathrm{ml}$ gelatin albumin was heat dissolved in PBS at $60^{\circ} \mathrm{C}$, followed by the addition of $0.3 \mathrm{~g} / \mathrm{ml}$ bovine serum albumin and $0.2 \mathrm{mg} / \mathrm{ml}$ sucrose. The solution was sterile filtered using $0.45 \mu \mathrm{m}$ filter (Sartorius) and stored at $-20^{\circ} \mathrm{C}$.

Mowiol: $5 \mathrm{~g}$ Mowiol was dissolved in $20 \mathrm{ml}$ PBS and stirred overnight. Afterwards $10 \mathrm{ml}$ glycerol was added and the solution again stirred overnight. After centrifugation for $30 \mathrm{~min}$ at $20,000 \mathrm{~g}$, the undissolved Mowiol was 
collected and removed and the supernatant was set to $\mathrm{pH} 7$. The solution was aliquoted and stored at $-20^{\circ} \mathrm{C}$.

For sectioning the embryos were mounted in gelatin/albumin, which was solidified by addition of glutaraldehyde. Sections of $30 \mu \mathrm{m}$ were cut using a Leica VT1000M vibratome and mounted on a glass slide in Mowiol.

\subsubsection{Protein standard methods}

\subsubsection{SDS-polyacrylamide gelelectrophoresis (SDS-PAGE)}

10x Laemmli buffer: 250mM Tris, $2.5 \mathrm{M}$ glycine, 0.1\% SDS

2x SDS loading buffer: $100 \mathrm{mM}$ Tris- $\mathrm{HCl} \mathrm{pH}$ 6.8, 4\% (w/v) SDS, 0.2\% (w/v) bromophenol blue, 20\% (v/v) glycerol, $200 \mathrm{mM} \beta$-mercaptoethanol

Proteins were separated according to their molecular weight by SDSpolyacrylamide gel electrophoresis (Laemmli, 1970). For denaturing of the proteins, samples were diluted with $2 x$ loading buffer and boiled for 3 min at $95^{\circ} \mathrm{C}$. For separation of the proteins, a $10 \%$ SDS gel was prepared according to standard protocols (Sambrook and Russel, 2001). The gel electrophoresis was performed in $1 \times$ Laemmli buffer and was started with a voltage of $80 \mathrm{~V}$. As soon the samples entered the separating gel the voltage was turned up to 200 $\mathrm{V}$. To determine the molecular weight of the proteins, a standard protein marker was used (Page Ruler Plus Prestained Protein Ladder, Fermentas).

\subsubsection{Western blotting}

Blocking solution: $5 \%(w / v)$ dry, non-fat milk powder in TBST

TBS: $50 \mathrm{mM}$ Tris, $150 \mathrm{mM} \mathrm{NaCl}, \mathrm{pH} 7.6$

TBST: $0.05 \%$ Tween-20 in TBS

After separation of the proteins by SDS-PAGE, the proteins were transferred to a nitrocellulose membrane by semi-dry blotting for $1 \mathrm{~h}$ in transfer buffer at 13-15 V (Sambrook and Russel, 2001; Towbin et al., 1979). After the transfer of the proteins the membrane was blocked in blocking solution for $1 \mathrm{~h}$ at RT. Incubation with the primary antibody was carried out overnight at $4^{\circ} \mathrm{C}$ in 
blocking solution. The following day, the membrane was washed 3 times for 10 min in blocking solution. The secondary antibody, which was coupled to a fluorescent IRdye (LI-COR), was applied to the blocking solution and the membrane was incubated for 1 hour at RT and afterwards washed twice for 10 min with blocking solution and once for $10 \mathrm{~min}$ with TBST at RT. To detect the fluorescent signals the LI-COR Odyssey Infrared Imaging system was used.

\begin{tabular}{|c|c|c|c|c|c|}
\hline Name & Company & Host & Type & Clonality & dilution \\
\hline anti-HA & Convance & mouse & primary & monoclonal & $1: 1000$ \\
\hline anti-GR & Santa Cruz & rabbit & primary & polyclonal & $1: 200$ \\
\hline anti-GAPDH & Abcam/Biozol & rabbit & primary & polyclonal & $1: 2500$ \\
\hline $\begin{array}{c}\text { anti-mouse } \\
800 \text { CW } \\
\text { IRdye }\end{array}$ & LI-COR & goat & secondary & polyclonal & $1: 15000$ \\
\hline $\begin{array}{c}\text { anti-rabbit } \\
680 \text { RD IRdye }\end{array}$ & LI-COR & goat & secondary & polyclonal & $1: 15000$ \\
\hline
\end{tabular}

Table 2.5 Summary of antibodies for western blot analysis 


\section{Results}

\subsection{Temporal and spatial expression analysis of $X$. laevis prdm14}

To gain insight into the function of prdm14 during early $X$. laevis embryogenesis, a detailed expression analysis was performed. The temporal expression of prdm14 was evaluated by semi-quantitative RT-PCR analysis using total RNA from staged embryos. As shown in Figure 3.1A, prdm14 transcripts are maternally provided and are maintained at high levels throughout tadpole stages.

The spatial expression of prdm14 was then determined by whole mount in situ hybridization. Prdm14 transcripts are detected in the animal hemisphere from early cleavage stages (stage 5, Fig. 3.1B) to blastula stages (stage 8, Fig. 3.1C). At early mid-gastrula stages (stage 10.5, Fig. 3.1D), prdm14 expression is confined to the dorsal ectoderm, which marks the prospective neuroectoderm. As shown in the sagittal section, prdm14 transcripts are restricted to the deep layer of the ectoderm (Fig. 3.1D'). At the end of gastrulation (stage 11.5, Fig. 3.1E), prdm14 is broadly expressed throughout the dorsal ectoderm with enrichment in the lateral domains, which most likely corresponds to the prospective neural plate border. At stage 12.5, prdm14 transcripts are present throughout the anterior neural plate (Fig. 3.1F). Posteriorly, the expression becomes refined to three longitudinal domains on both sides of the midline, which prefigures the territories of primary neurogenesis. Strong expression is observed in the intermediate and lateral domains, while the expression in the medial domain is weak (Fig. 3.1F). From stage 13 to 15, prdm14 expression in the posterior neural plate is maintained and becomes stronger in the medial stripe (Fig. 3.1G-I). As seen in transversal section of a stage 15 embryo (Fig. 3.1l'), the expression of prdm14 is restricted to the deep layer of the neuroectoderm, where the primary neurons arise (Hartenstein, 1989). At stage 13, the expression becomes restricted to the anterior neural plate border, the trigeminal-profundal placode and to a horseshoe shaped domain, which will give rise to the ventral midbrain (Eagleson and Harris, 1990) (Fig. 3.1G). 


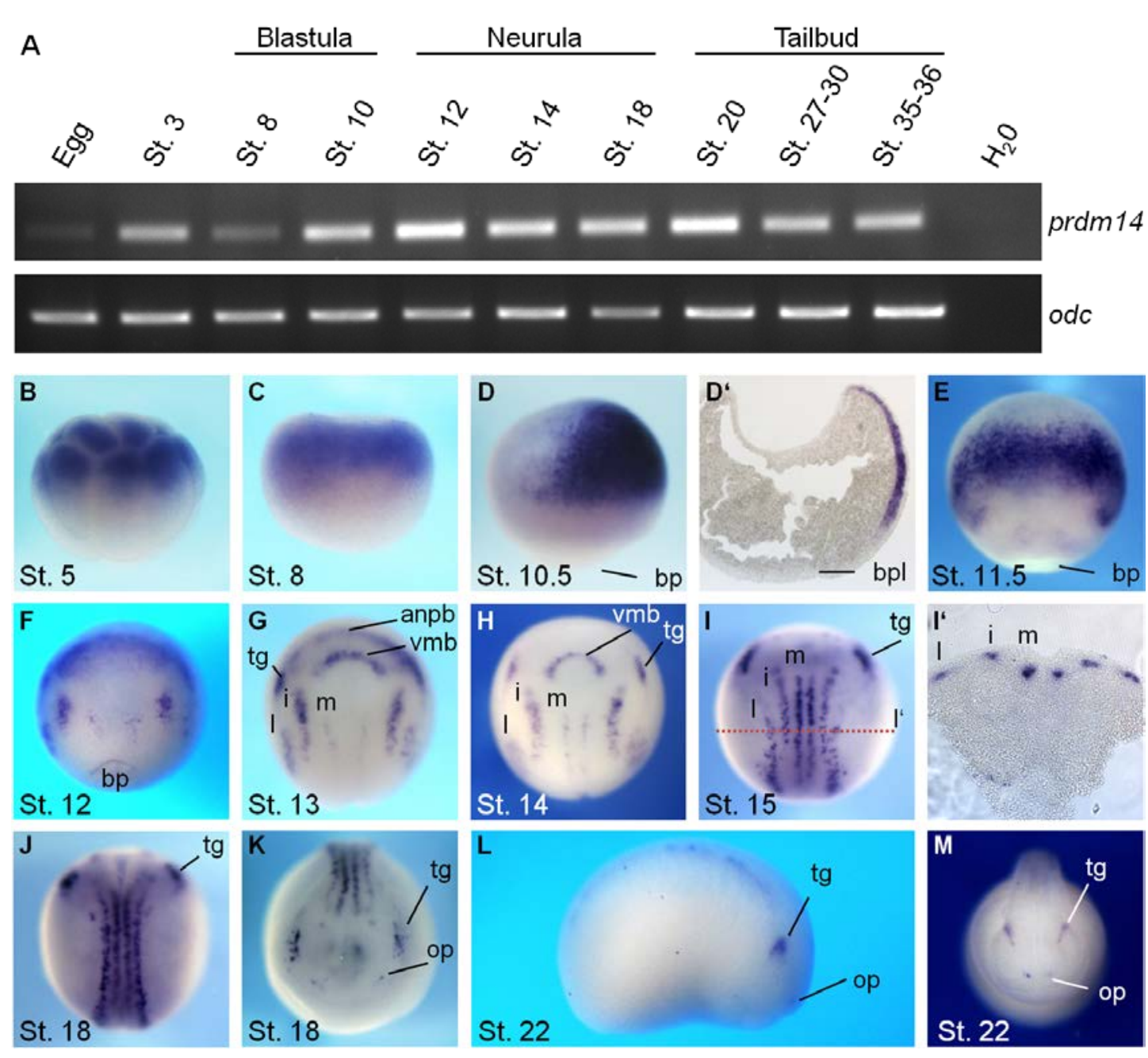

Fig. 3.1 Temporal and spatial expression analysis of prdm14 in $X$. laevis embryos. (A) Prdm14 is maternally expressed. Temporal prdm14 expression analysis by semi-quantitative RT-PCR at indicated stages. Expression levels were shown by odc expression. (B-M) Prdm14 is expressed throughout the developing nervous system. Spatial expression analysis of prdm14 in $X$. laevis embryos by whole mount in situ hybridization. (B-D) Lateral view of the embryos, animal up. (D') Sagittal section of embryo shown in D. (E-J) Dorsal view of embryos, anterior up. (I') Transverse section at indicated level of embryo shown in I. (K, M) Anterior view of embryo, dorsal up. (L) Lateral view of embryo, anterior right, dorsal up. anpb, anterior neural plate border; bp, blastoporus; bpl, blastoporus lip; i, intermediate; I, lateral; m, medial; op, otic placode; tg, trigeminal-profundal placode; vmb, ventral midbrain

During neurulation, the expression of prdm14 is maintained in the posterior primary neurons, the trigeminal-profundal placode and olfactory placodes (Fig. 3.1J-M). At stage 26, prdm14 transcripts are detected in the trigeminal placodes, otic vesicles, olfactory placodes and throughout the spinal cord (Fig 3.2A). Transverse sections show that prdm14 transcripts are present in the inner segment of the otic vesicle and in the marginal zone of the neural tube where postmitotic neurons reside (Fig. 3.2A'-A'”) (Bellefroid et al., 1996). In the anterior neural tube, prdm14 expression is restricted to two distinct domains (Fig. 3.2A"), while more posteriorly the expression spans the entire marginal zone of the neural tube (Fig. 3.2A"'). Double whole mount in situ hybridization 
for prdm14 and tubb2b shows that the expression domains for both genes overlap in the marginal zone throughout the neural tube, confirming that prdm14 indeed is expressed in postmitotic neurons (Fig. 3.2B-B").

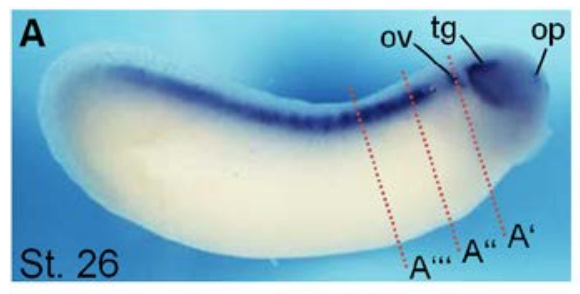

B

\section{tubb2b/prdm 14}

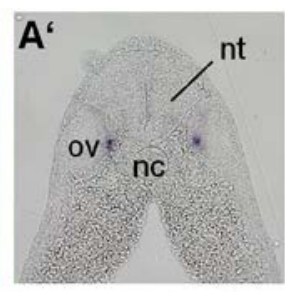

$\mathbf{B}^{\mathbf{6}}$

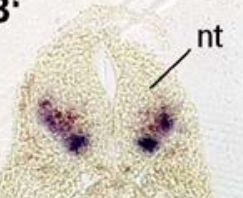

ne

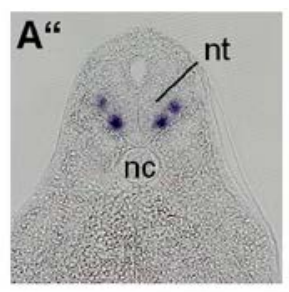

B“"
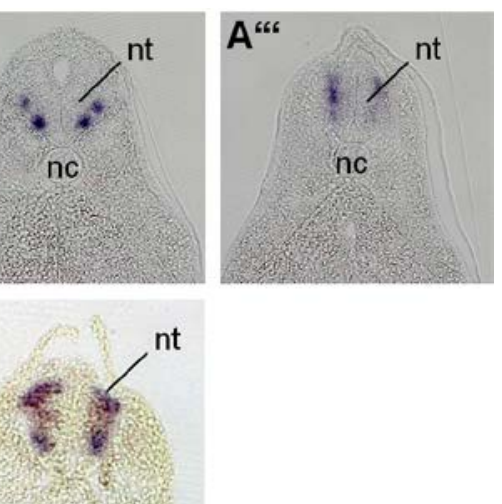

nc

Fig. 3.2 Prdm14 is expressed in the marginal zone of the neural tube. (A-B") Spatial expression analysis of prdm14 in $X$. laevis stage 26 embryos by whole mount in situ hybridization. (A, B) Lateral view of embryo (anterior right). (A'-A'"') Transverse sections at indicated levels of embryo shown in A. (B-B') Double whole mount in situ hybridization of stage 26 embryos for tubb2b (red) and prdm14 (purple). (B'-B') Transverse sections at indicated levels of embryo shown in B. nc, notochord; nt, neural tube; op, olfactory placode; ov, otic vesicle; tg, trigeminal profundal placode

Taken together, the expression of prdm14 is strongly indicative for a role during the early development of the nervous system. While prdm14 is clearly present in the post-mitotic neurons of the neural tube, it is also expressed in the neural plate when the proneural genes specify the primary neurons. To further clarify the timing of prdm14 expression in relationship to the proneural genes neurog1 and neurog2, to which prm14 is most similar, a comparative expression analysis was performed. Both prdm14 and neurog2 are expressed throughout the prospective neuroectoderm at the onset of gastrulation (stage 10), with the expression of neurog 2 being weaker and located closer to the blastopore (Fig. 3.3). At the end of gastrulation (stage 12), transcripts of neurog1 and neurog2 are detectable in the posterior neural plate in three distinct domains prefiguring the territories of primary neurogenesis. At this stage prdm14 transcripts are only present in the medial and lateral stripes of primary neurogenesis and the anterior neural plate. At stage 14, prdm14, neurog1 and neurog2 are all expressed in the trigeminal-profundal placodes as well as in the three bilateral longitudinal domains of primary neurogenesis. However, the expression of prdm14 and neurog1 are strikingly similar. They are both expressed in the future ventral midbrain, and compared to neurog2, their 
expression in the longitudinal domains is more refined and extends more anteriorly. During neurulation (stage 18) all transcripts are present in the neural fold and in the trigeminal-profundal placodes.

In summary, prdm14 differs from the analyzed proneural genes as it is expressed early and strongly in the anterior neural plate. In the territories of primary neurogenesis of the posterior neural plate, the expression of neurog 2 prefigures that of prdm14.
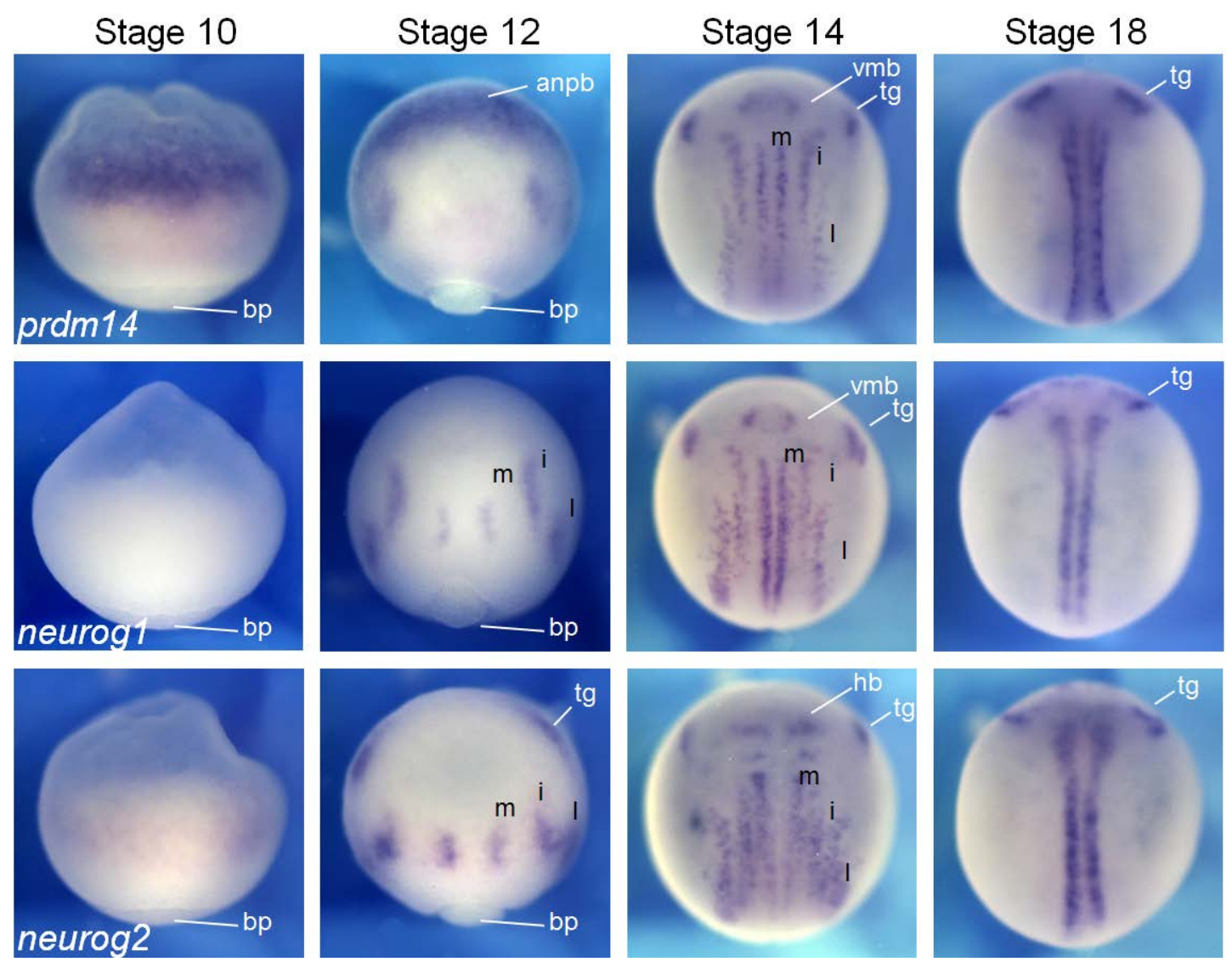

Fig. 3.3 Prdm14, neurog1 and neurog2 are co expressed in territories of primary neurogenesis. Comparative expression analysis of stage matched embryos. Stage 10: lateral view, animal up; Stage 12-18: dorsal view, anterior up. anpb, anterior neural plate border; bp, blastoporus; hb, hindbrain; i, intermediate; I, lateral; m, medial; tg, trigeminal-profundal placode; vmb, ventral midbrain

The temporal expression of prdm14 in the neural plate and in the outer mantel layer of the neural tube suggests a function for Prdm14 downstream of Neurog2. Therefore, the regulation of prdm14 by the proneural transcription factor Neurog2 was analyzed (Ma et al., 1996). Neurog2 mRNA was injected together with $\beta$-gal mRNA to indicate the cells that have received the injected material, into one blastomere of two-cell stage embryos. The embryos were analyzed by whole mount in situ hybridization at neural plate stage (Fig. 3.4A). 
As anticipated, microinjection of neurog2 mRNA strongly induces expression of the postmitotic neuronal marker tubb2b (also known as $n$-tubulin) in the deep layer of the ectoderm ( $n=13,100 \%$ ectopic expression) (Fig. 3.4 B-B') (Ma et al., 1996, Chalmers et al., 2002). Strong ectopic expression was similarly observed for prdm14 ( $n=28,100 \%$ ectopic expression) (Fig. 3.4D-D').

Prdm14 was identified as a putative target gene of the repressor protein Hes5.1, which itself is a target of the Notch pathway (Klisch, 2006, Kiyota et al., 2001). Therefore, the influence of Notch signaling on prdm14 expression was also analyzed. mRNA encoding for the Notch intracellular domain (NICD), which is the constitutively active version of the Notch receptor, was injected into one blastomere of two-cell stage embryos. Consistent with the known activity as a negative regulator of primary neurogenesis (Chitnis and Kintner, 1996), NICD mRNA injection resulted in the inhibition of $t u b b 2 b$ on the injected side of the embryo ( $n=19,100 \%$ downregulated). NICD also strongly inhibited the expression of prdm14 ( $\mathrm{n}=31,90 \%$ downregulated) (Fig. 3.4C-E). Taken together, the expression and regulation of prdm14 suggests a role for prdm14 in primary neurogenesis in $X$. laevis.
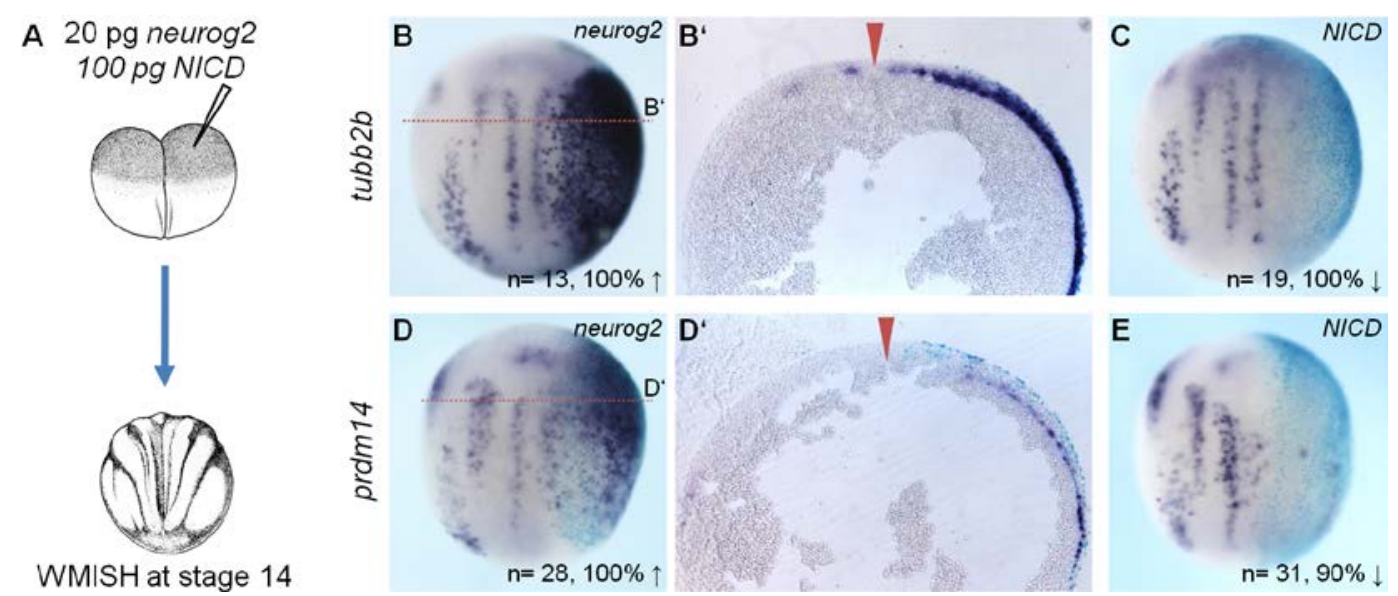

Fig. 3.4 Prdm14 expression is regulated by key regulators of neurogenesis. (A) Neurog2 $(20 \mathrm{pg})$ or NICD (100 pg) mRNA together with $\beta$-Gal (75 pg) mRNA (light blue staining) were injected into one blastomere of a two-cell stage embryo. (B-E) Gene expression was analyzed by whole mount in situ hybridization using markers indicated on the left side. The injected side is on the right, dorsal view, anterior up. (B', D') Transverse sections at indicated levels of embryo shown in $B$ and $D$, respectively. Red arrows indicate the midline. 


\subsection{Prdm14 gain of function}

To gain further support for a role of prdm14 during $X$. laevis primary neurogenesis, a gain of function approach was performed. mRNA encoding a HA-tagged version of prdm14 (500 pg) was microinjected into one of two blastomeres and the influence on neuronal differentiation evaluated by whole mount in situ hybridization. At neural plate stage, the expression of tubb2b was inhibited on the prdm14 mRNA injected side of the embryo $(n=36,64 \%$ downregulated) (Fig. 3.5B). To ensure that the observed phenotype was not due to an unspecific effect, gfp mRNA was injected at the same concentration as prdm14-HA. Microinjection of gfp mRNA did not affect tubb2b expression, which suggested that any observed phenotypes could be attributed to the injected prdm14 mRNA (Fig. 3.5A).

The inhibition of neuronal differentiation by prdm14 was only transient, as in the neural tube of tailbud stage embryos the expression of $t u b b 2 b$ was recovered (Fig. 3.5C-D'). Since the transient inhibition may be due to low protein stability, a GR-tagged version of prdm14 was generated, as GR-fusion proteins often exhibit an increased effectiveness due to higher protein stability (Kolm and Sive, 1995). The mRNA encoding for prdm14-GR was injected into one blastomere of two-cell stage embryos and the activity of the fusion protein was induced by addition of dexamethasone (dex) at the four-cell stage (stage 3). Embryos that were injected with prdm14-GR mRNA and treated with dex showed an inhibition of $t u b b 2 b$ at the neural plate stage. Inhibition of $t u b b 2 b$ was not observed in injected and untreated embryos, demonstrating that Prdm14-GR is indeed hormone inducible (Fig. 3.5E). The phenotype induced by prdm14-GR mRNA is similar to that of prdm14-HA mRNA injected embryos ( $\mathrm{n}=$ $39,64 \%$ downregulated). However, the inhibition of neuronal differentiation was observed at a higher frequency ( $n=17,100 \%$ downregulated) (Fig. 3.5F). Moreover, Prdm14-GR induced ectopic tubb2b expression in the non-neural ectoderm of tailbud stage embryos ( $n=23$, 83\% ectopic expression) (Fig. 3.5G$H^{\prime}$, red arrows). In the transverse section, $t u b b 2 b$ expression was strongly increased and the entire dorsal region of the neural tube was enlarged compared to the uninjected side (Fig. 3.5H'). The strongest expansion was observed in the dorsal half of the neural tube. Interestingly, a thickening of mesenchymal tissue on the injected side of tailbud stage embryos was also 
observed, which was more prominent in the prdm14-GR injected embryos than in the prdm14-HA injected ones (Fig. 3.5D', H', black brackets).

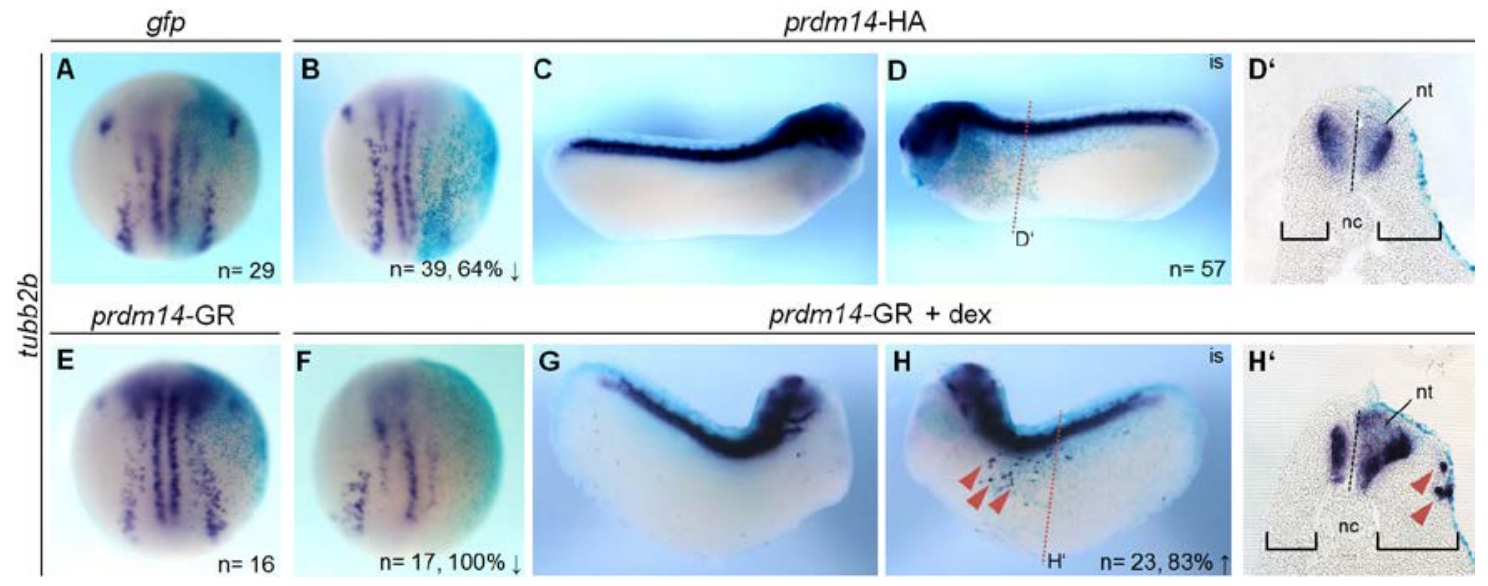

Fig. 3.5 Prdm14-GR overexpression induces stronger phenotypes. (A-H') Indicated mRNA was injected together with $\beta$-Gal (75 pg) mRNA (light blue staining) into one blastomere of a two-cell stage embryo. tubb2 $b$ expression was analyzed by whole mount in situ hybridization. Injected side is always to the right. (A, B, E, F) Neural plate stage, dorsal view, anterior up; (C, D, G, H) tailbud stage, lateral view, dorsal up; (A) 500 pg MT-gfp mRNA; (B-D') 500 pg prdm14HA; (E) 500 pg prdm14-GR; (F-H') 500 pg prdm14-GR, embryos were treated from four-cell stage on with dex; (D', H') Transverse sections at indicated levels of embryo shown in D and $H$ respectively. Black brackets indicate width of mesenchymal tissue at the level of the notochord. Dashed line indicates midline of the neural tube.is, injected side; nt, neural tube; nc, notochord

\subsection{Prdm14 promotes proliferation and the expansion of neural progenitors}

Prdm14 overexpression at neural plate stage inhibited the expression of the postmitotic neuron marker tubb2b (Fig. 3.5B, F) and at tailbud stages resulted in an enlarged neural tube and ectopic neuronal differentiation (Fig. $\left.3.5 \mathrm{H}^{\prime}\right)$. To analyze if these effects were caused by the expansion of progenitor cells, the effect of prdm14-GR overexpression on the neural plate marker sox2 was evaluated, as it is a definitive marker of neural progenitors (Graham et al., 2003; Ellis et al., 2004) (Fig. 3.6A). Microinjection of prdm14-GR results in the expansion of the sox 2 expression domain on the injected side of the embryo ( $n=20 ; 75 \%$ increased) (Fig. 3.6B).

To determine if the expansion of sox 2 expression by prdm14-GR is the result of increased proliferation, the number of mitotically active cells in the dorsal ectoderm was quantified by staining for phosphorylated histone $3(\mathrm{pH} 3)$ (Dent et al., 1989) (Fig. 3.6C). To quantify the number of mitotically active cells, three embryos were sectioned and the pH3 positive cells were counted in the dorsal halves of 15 consecutive sections. The number of mitotically active cells 
in the neuroectoderm was increased almost two-fold on the prdm14-GR injected side compared to the uninjected side (Fig. 3.6C'-D). These results suggest that prdm14 promotes the proliferation of progenitor cells, resulting in the inhibition of neuronal differentiation at neural plate stage and in an enlarged neural tube in later stages.
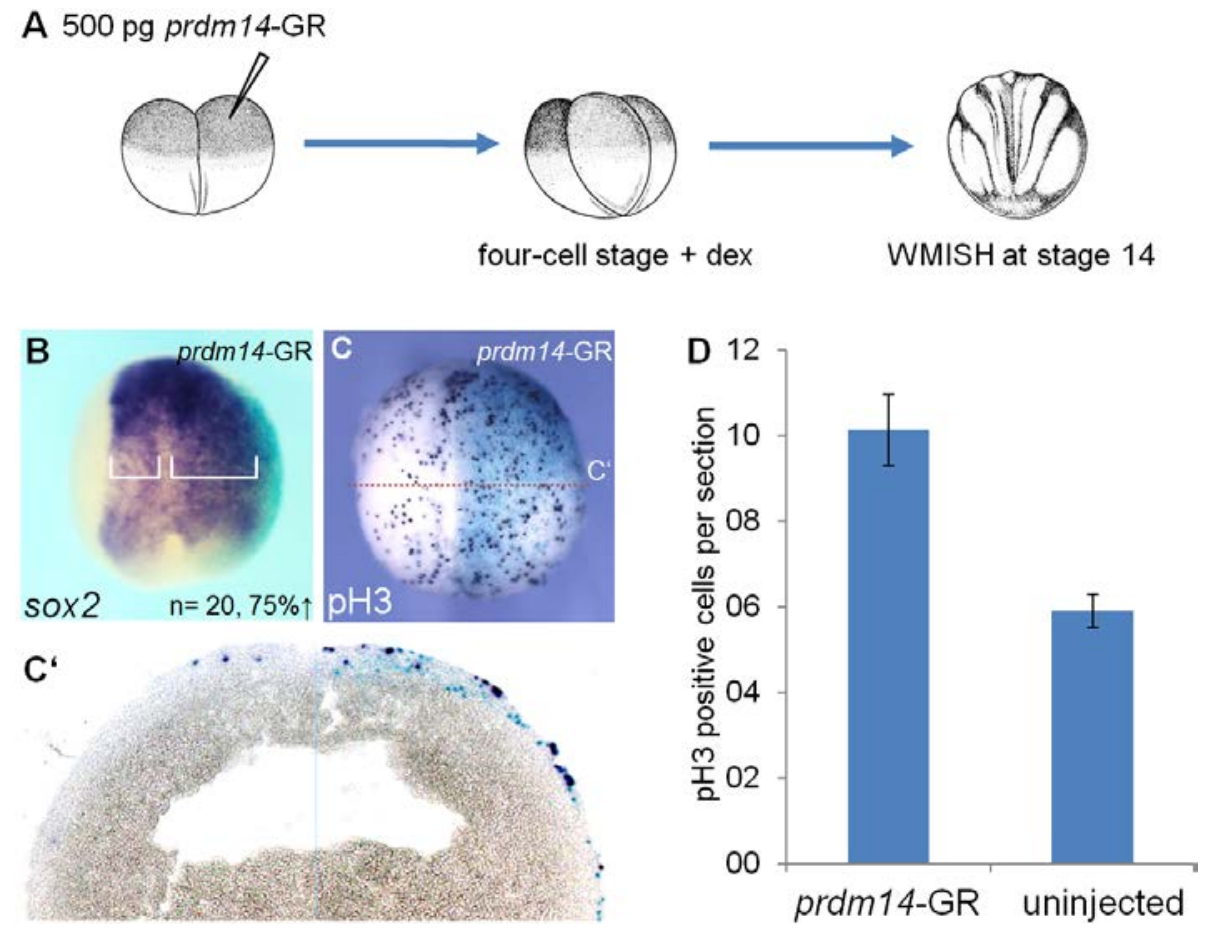

Fig. 3.6 Prdm14-GR overexpression promotes proliferation. (A) Prdm14-GR (500 pg) mRNA together with $\beta$-Gal (75 pg) mRNA (light blue staining) were injected into one blastomere of a two-cell stage embryo. Embryos were treated from four-cell stage on with dex. (B-C) Gene expression was analyzed at stage 14 by whole mount in situ hybridization using markers indicated on the bottom left side. The injected side is on the right, dorsal view, anterior up. (B) The white brackets indicate width of expression domain. (C') Transverse section at indicated levels of embryo shown in C. (D) The graph shows the statistical evaluation of 15 consecutive sections of three embryos based on the number of $\mathrm{pH} 3$ positive cells in the dorsal half on the injected and uninjected site as indicated in the cross-section $C^{\prime}$. Error bars represent the standard error of the mean of two independent experiments (+/-SEM). 


\subsection{Prdm14 promotes ectopic sensory neuron formation}

Prdm14-GR induces ectopic neurons in the non-neural ectoderm of tailbud stage embryos (Fig. 3.5G-H') raising the question as to which neuronal subtype they belong to. As the dorsal neural tube, which arises from the neural plate border (NPB), was strongly expanded, the expression of $t / x 3$ was analyzed, which is expressed in the NPB and the dorsally located glutamatergic Rohon-Beard sensory neurons (Patterson and Krieg, 1999; Rossi et al., 2009). Whole mount in situ hybridizations of embryos overexpressing prdm14-GR showed ectopic expression of $t \mid x 3$ in the non-neural ectoderm $(n=22 ; 76 \%$ ectopic staining) (Fig. 3.7C, red arrows) and an increased expression in the dorsal neural tube (Fig. 3.7C', red arrows). The glutamatergic neurotransmitter identity of these neurons was further supported by the ectopic expression of the vesicular glutamate transporter 1 s/c17a7 (vglut1) ( $n=25 ; 80 \%$ ectopic staining) (Fig 3.7D-E'). To determine if other ectopic neuronal subtypes are induced by Prdm14-GR, the expression of $m n \times 1$ (also known as $h b 9$ ) was analyzed, which is expressed in cholinergic motor neurons in the most ventral aspect of the neural tube (Saha et al., 1997). Ectopic $m n \times 1$ expression was never observed in the non-neural ectoderm. Nonetheless, an expansion of the endogenous expression domain was observed, which may be the result of an overall enlargement of the neural tube caused by prdm14-GR overexpression (Fig. 3.7F-G'). 


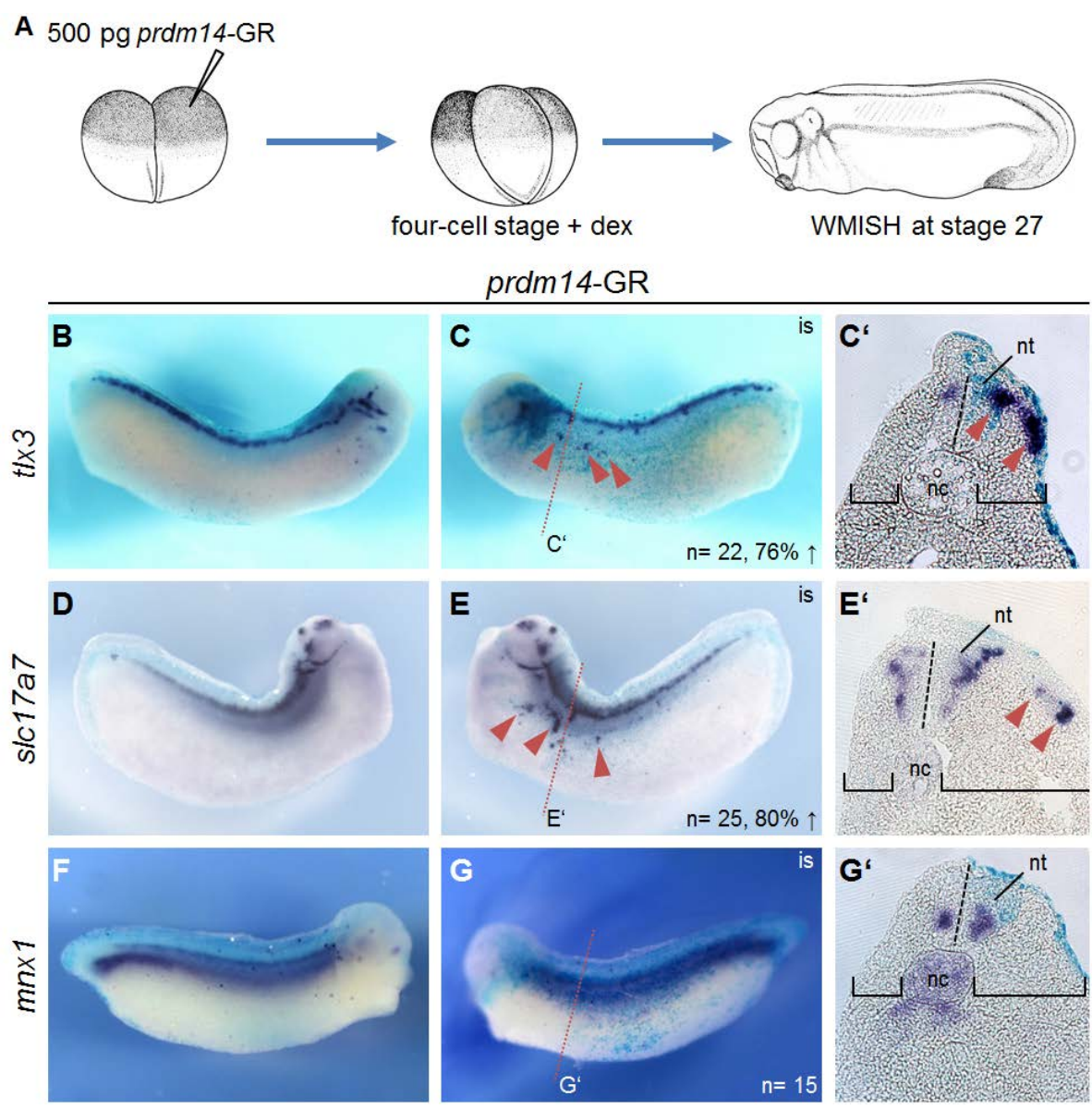

Fig. 3.7 Prdm14-GR overexpression promotes sensory neurons in tailbud stage embryos. (A) Prdm14-GR (500 pg) mRNA together with $\beta$-Gal (75 pg) mRNA (light blue staining) were injected into one blastomere of a two-cell stage embryo. Embryos were treated from four-cell stage on with dex. (B-G') Gene expression was analyzed at stage 27 by whole mount in situ hybridization using markers indicated on the left. The injected side is on the right, lateral view. is, injected side (C', E', G') Transverse sections at indicated levels of embryo shown in C, E, G respectively. Black brackets indicate width of mesenchymal tissue at the level of the notochord. Dashed line indicates midline of the neural tube. nt, neural tube; nc, notochord

To determine if prdm14 overexpression is sufficient to induce glutamatergic sensory neurons in pluripotent cells, the animal cap assay was used. Control and prdm14-GR mRNA-injected embryos were treated with dex at stage 3 and animal caps were isolated from blastula stage embryos (Fig. 3.8A). At the equivalent of neural plate stage (stage 14) and tailbud stage (stage 27) total RNA was isolated and analyzed by RT-PCR. Similar to the phenotype in embryos, at the equivalent of tailbud stages, tubb2b as well as $t / x 3$ were induced. These markers were not observed in prdm14-GR mRNA-injected animal caps not treated with dex, or control uninjected caps treated with dex. To elucidate if prdm14 directly promotes neural differentiation or requires the activity of proneural genes, the expression of neurog2 was analyzed. At the equivalent of stage 14, neurog2 is induced in caps expressing Prdm14-GR. The 
absence of tubb2b at the equivalent of neural plate stage is similar to the transient inhibition of neurogenesis observed in the embryos. The regulation of neurog2 by Prdm14-GR is unexpected as neurog2 strongly promotes prdm14 (Fig. 3.4B), suggesting a positive feedback loop between these two factors. Hence, active Prdm14-GR is able to promote neuronal differentiation in whole embryos as well as animal caps.

A $\quad 500$ pg prdm14-GR

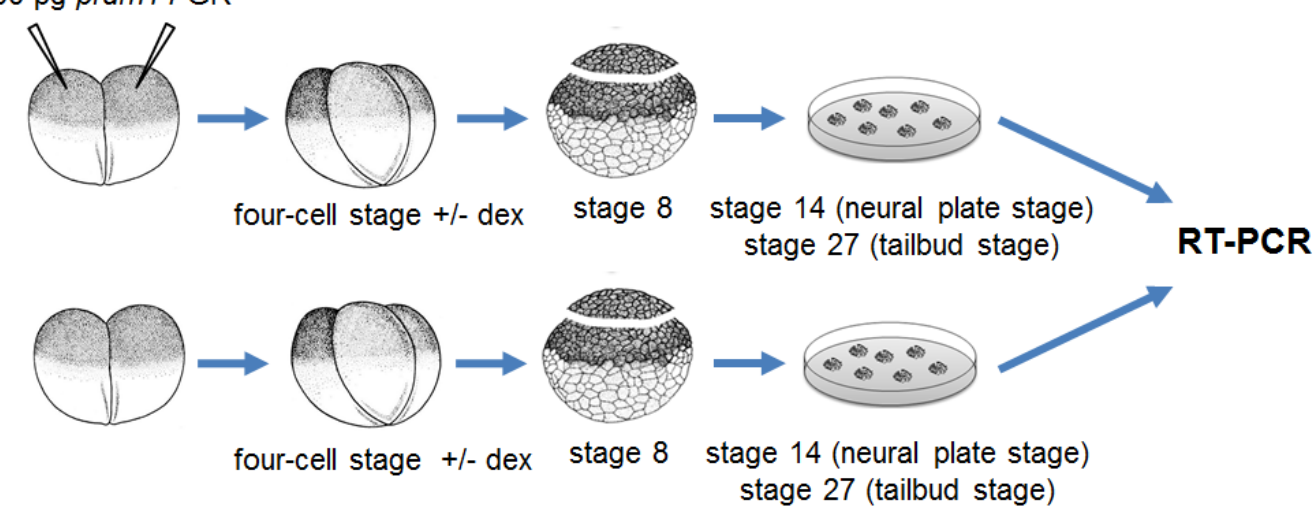

B

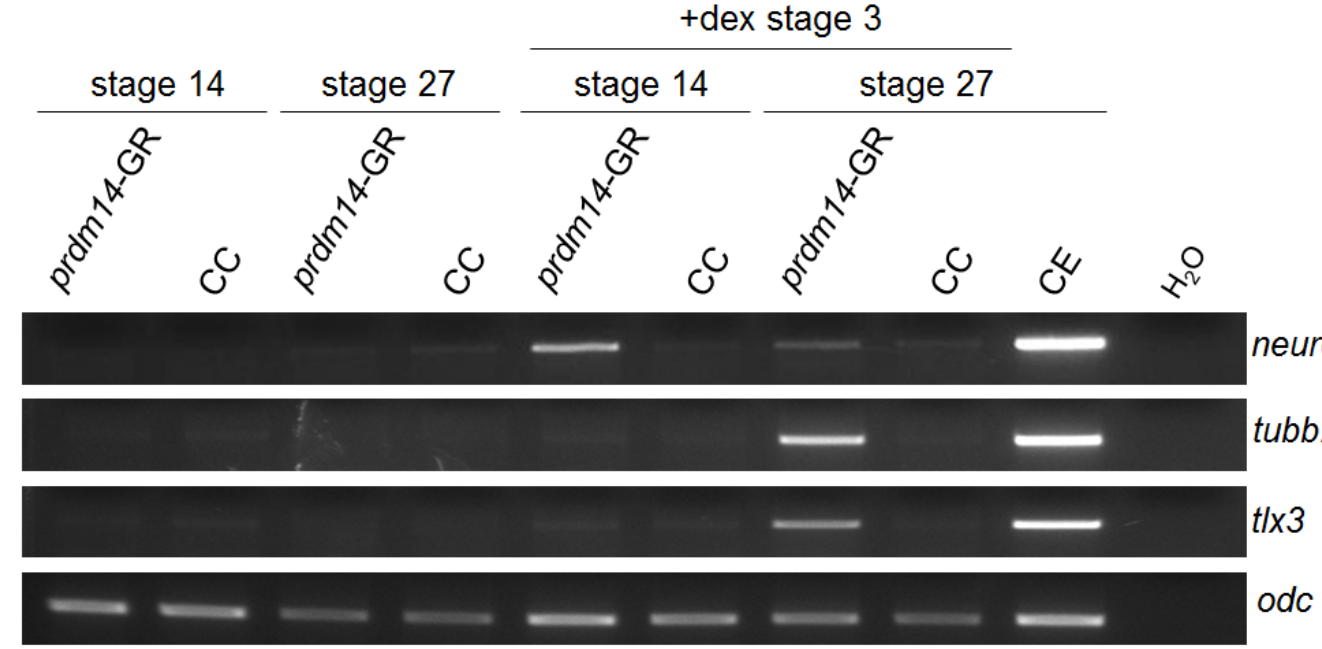

Fig. 3.8 Prdm14-GR overexpression activates sensory neuron marker $t / x 3$ in animal caps. (A-B) Prdm14-GR (500 pg) mRNA was injected into both blastomeres of a two-cell stage embryo. Control and prdm14-GR mRNA-injected embryos were treated with dex from four-cell stage on and animal caps were isolated from blastula stage embryos. At the equivalent of neural plate stage (stage 14) and tailbud stage (stage 27) total RNA was isolated and analyzed by RT-PCR using markers indicated on the right. Expression levels were shown by odc expression. 


\subsection{Knock down of Prdm14}

To further analyze the role of Prdm14 in the regulation of neuronal differentiation, loss of function studies were performed. Antisense morpholino oligonucleotides were proven as an effective tool in $X$. laevis for the inhibition of gene function (Heasman et al., 2000; Nutt et al., 2001; Heasman, 2002). However, their use in $X$. laevis is complicated by the fact that $X$. laevis is allotetraploid and therefore redundancies might exist. For prdm14 two sequence-related paralogs in $X$. laevis exists, prdm14a and prdm14b. In order to efficiently knock down the expression of prdm14, two splicing morpholinos (SpMO) were simultaneously used to disrupt the splicing of prdm14a and prdm14b. Both SpMOs target the exon1-intron1 boundary to prevent the splicing of intron1 (Fig. 3.9A-B). If intron1 is retained, only a truncated protein should be translated, as the intron 1 contains a stop codon. The resulting truncated Prdm14 proteins would have 208 amino acids (Prdm14a) and 210 amino acids (Prdm14b), and would not contain the PR and Zn finger domains (Fig. 3.9C).

To test the specificity and function of the SpMOs, they were injected alone or together at increasing concentrations $(5,10,25 \mathrm{ng})$ into both blastomeres of a two-cell stage embryo. The embryos were collected at stage 14 and RT-PCR analysis was performed using primers which bind within exon1 and exon2 of prdm14a and prdm14b. Knock down of prdm14 by both SpMOs led to a concentration-dependent upward shift of the amplicon bands indicating a larger size (Fig. 3.9D). As verified by sequencing analysis of these amplicons, the increase in size was due to the impaired splicing resulting in intron 1 retention. While Prdm14a SpMO was specific for prdm14a, the Prdm14b SpMO impaired the splicing of both prdm14a, and prdm14b. However, Prdm14b was more effective on inhibiting prdm14b splicing. Analysis of tailbud stage embryos injected with both SpMOs demonstrated that splicing is efficiently inhibited at later stages of development. Hence, the Prdm14 SpMOs are an effective tool to knockdown Prdm14. 

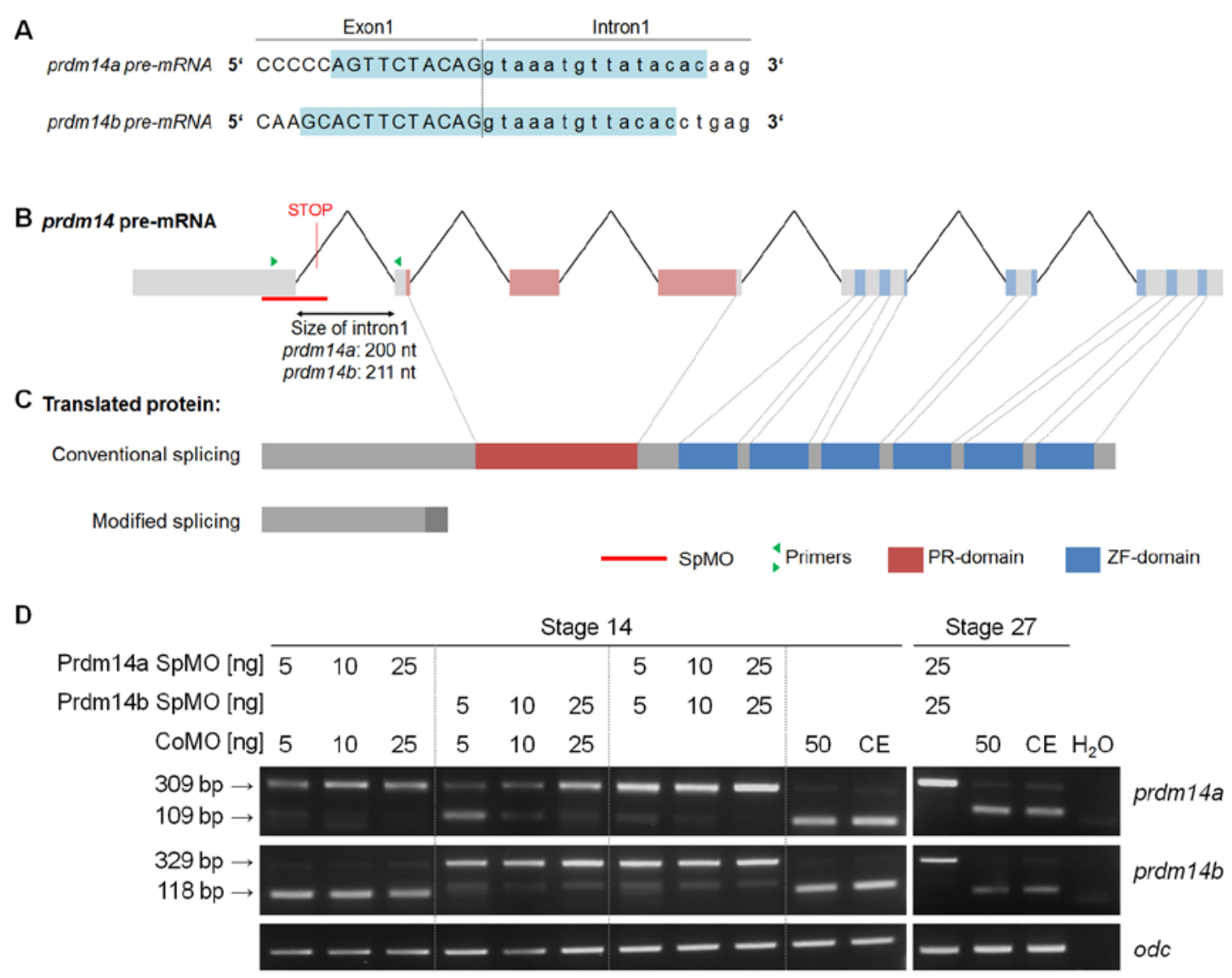

Fig. 3.9 Injection of Prdm14 splicing morpholinos causes intron1 retention. (A) Nucleotide sequence of prdm14a and prdm14b exon1/intron1 boundary. SpMO binding sites are highlighted in blue. (B) Schematic representation of prdm14 pre-mRNA. (C) Schematic representation of the translated protein after conventional splicing and intron1 retention. (D) Morpholino oligonucleotides were injected in indicated combinations into both blastomeres of two-cell stage embryos. At the equivalent of neural plate stage (stage 14) and tailbud stage (stage 27) total RNA was isolated and analyzed by RT-PCR using markers indicated on the right. Expression levels were shown by odc expression. Expected amplicon sizes are indicated on the left.

To determine how prdm14 loss of function would affect neuronal differentiation at tailbud stage, Prdm14 was knocked down by SpMOs in one dorsal blastomere of four-cell stage embryos (Fig. 3.10A). As shown by whole mount in situ hybridization, the loss of Prdm14 had no influence on the expression of tubb2b and $t / x 3$ (Fig. 3.10B-E'). Next, the influence of Prdm14 knock down on motor neuron axonal outgrowth in the spinal cord was analyzed, since impairments thereof have been reported in zebrafish Prdm14 morphants (Liu et al., 2012). Therefore, both morpholinos where injected into both dorsal blastomeres of 4-cell stage embryos to specifically target the SpMOs to the neural tube (Fig. 3.10F). As shown by immunofluorescence staining against acetylated Tubulin, the motor neuron axon outgrowth is not impaired by the control morpholino (Fig. 3.10G) or Prdm14 knock down (Fig. 3.10H). In 
summary, while overexpression of prdm14 leads to the ectopic formation of neurons of a specifically glutamatergic character, the knock down of Prdm14 has no effect on the specification of sensory neurons and neuronal differentiation.
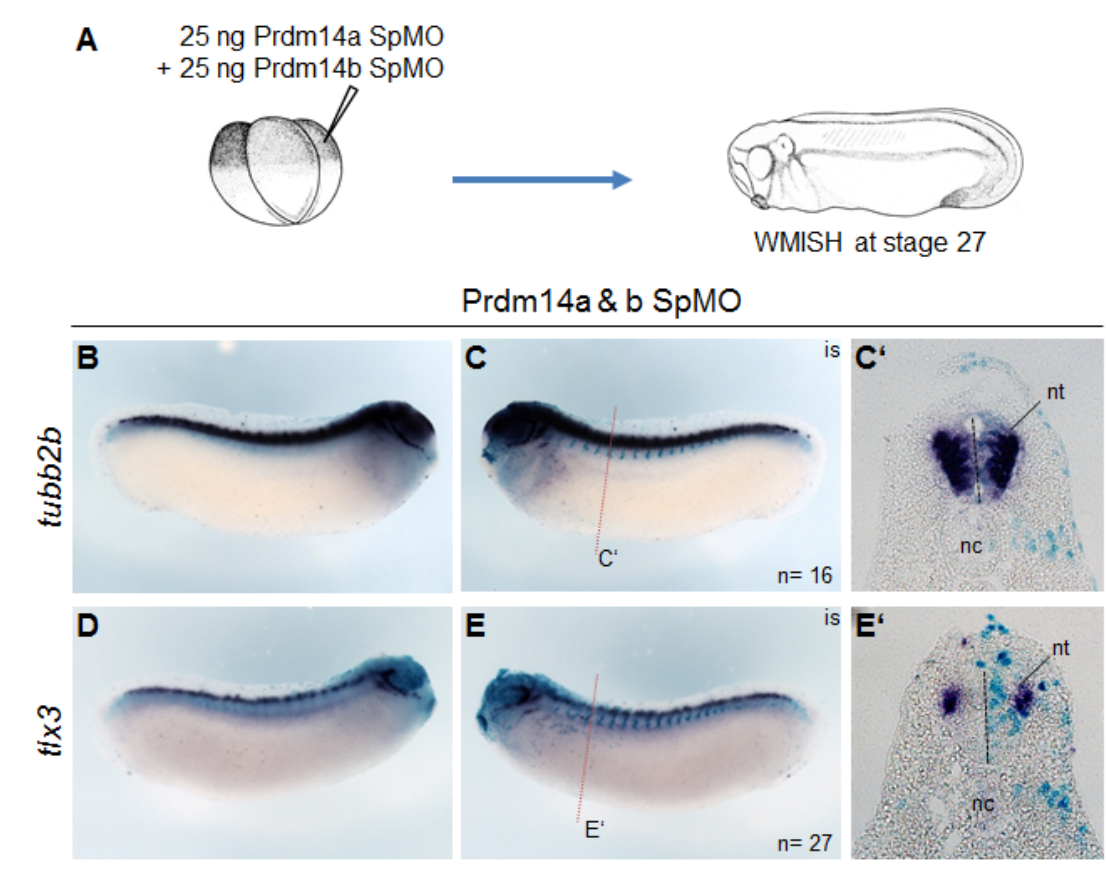

F $\quad 25 \mathrm{ng}$ Prdm14a SpMO

+25 ng Prdm14b SpMO
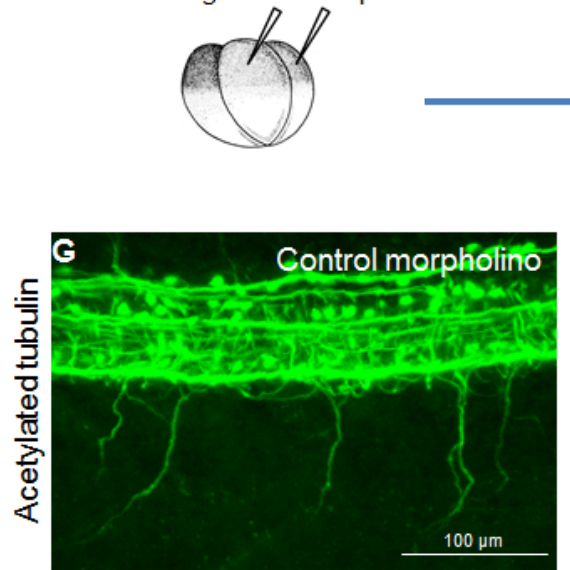

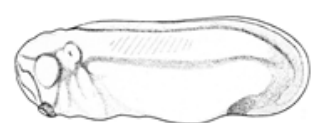

Fluorescent immunostaining at stage 27

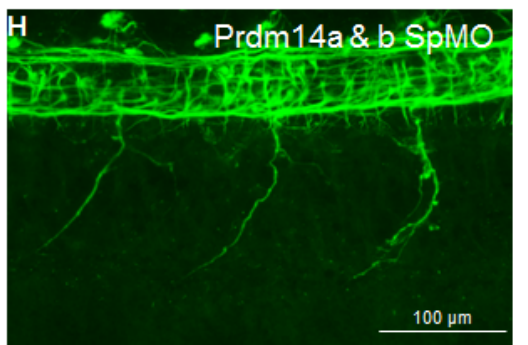

Fig. 3.10 Knock down of Prdm14 does not influence neuronal differentiation and axon outgrowth of motor neurons. (A) Prdm14a SpMO and Prdm14b SpMO (25 pg each) together with $\beta$-Gal (75 pg) mRNA (light blue staining) were injected into one dorsal blastomere of fourcell stage embryos. (B-E') Gene expression was analyzed at stage 27 by whole mount in situ hybridization using markers indicated on the left. The injected side is on the right, lateral view. is, injected side (C', E') Transverse sections at indicated levels of embryos shown in C and E, respectively. Dashed line indicates the midline of the neural tube. nt, neural tube; nc, notochord (F) Prdm14a SpMO and Prdm14b SpMO (25 pg each) or control morpholino (50 ng) were injected into both dorsal blastomeres of four-cell stage embryos. (G-H) Axon outgrowth of motor neurons was visualized by fluorescent immunostaining against acetylated tubulin at stage 27 . 


\subsection{Identification of Prdm14 downstream targets}

To further investigate the function of Prdm14 during early development, gain of function approaches were employed. Overexpression of prdm14-GR was sufficient to induce glutamatergic neurons in pluripotent animal cap cells. Hence, we decided to use this system to identify the global regulatory gene network induced by Prdm14 through RNA-sequencing analysis (Fig. 3.11A). Control and prdm14-GR mRNA-injected embryos were treated with dex at fourcell stage and animal caps were isolated from blastula stage embryos. At the equivalent of neural plate stage (stage 14) and tailbud stage (stage 27) total RNA was isolated and reverse transcribed into cDNA, which was subjected to single read (50 bp) sequencing. The sequence reads were aligned to the genome reference sequence of $X$. tropicalis (Joint Genome Institute assembly v4.2) using the STAR alignment software (Dobin et al., 2012), allowing 5 mismatches within 50 bases. Using this approach in total $86-89 \%$ of the reads could be annotated. Genes were considered as candidates if they showed at least a 2-fold change (log2FC>1) over control caps and a FDR-corrected pvalue $<0.05$ 
A 500 pg prdm14-GR

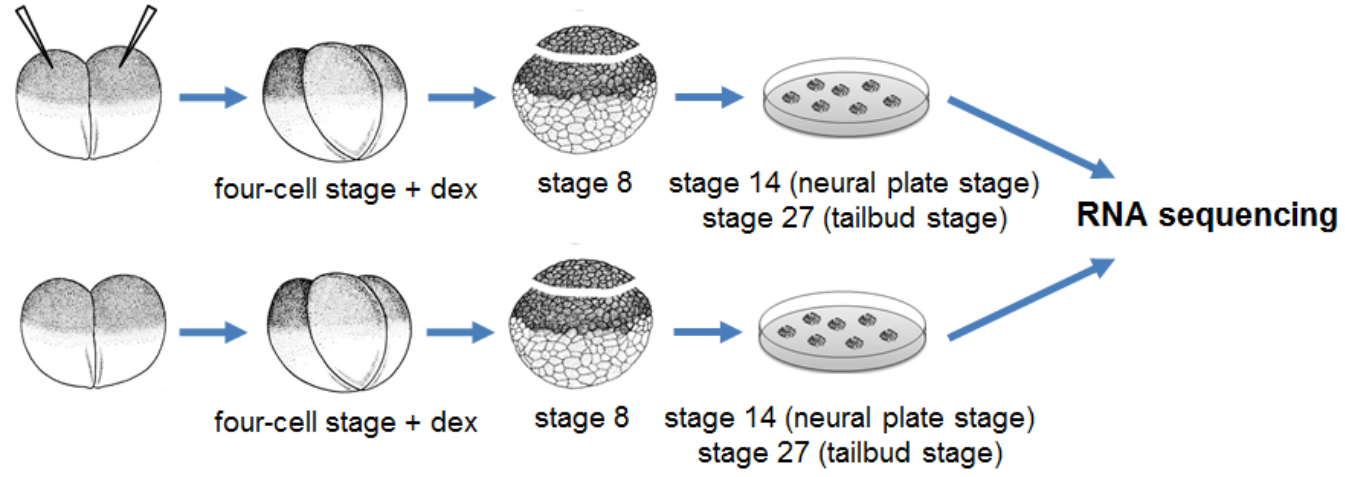

B

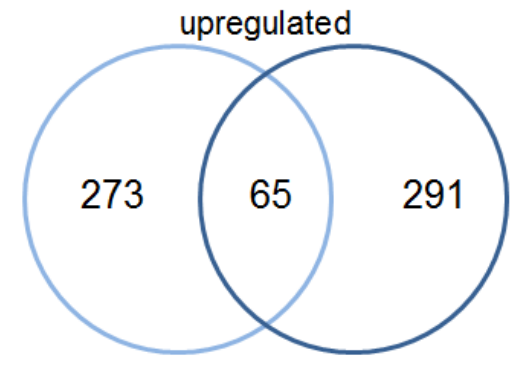

Stage $14 \quad$ Stage 27

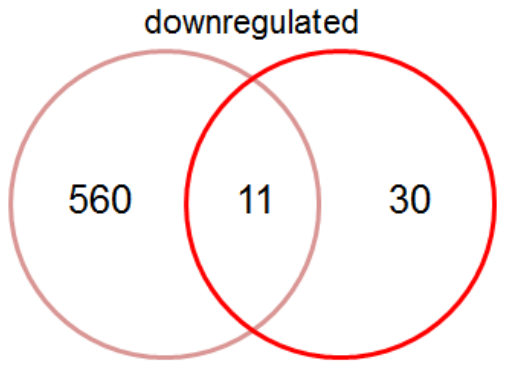

Stage $14 \quad$ Stage 27

\begin{tabular}{|l|c|c|}
\hline \multirow{2}{*}{$\begin{array}{l}\text { log2 fold } \\
\text { change }\end{array}$} & \multicolumn{2}{|c|}{ prdm14-GR vs. CC } \\
\cline { 2 - 3 } & Stage 14 & Stage 27 \\
\hline$\uparrow>5$ & 18 & 46 \\
\hline$\uparrow>3$ & 68 & 208 \\
\hline$\uparrow>2$ & 140 & 296 \\
\hline$\uparrow>1$ & 338 & 356 \\
\hline
\end{tabular}

\begin{tabular}{|l|c|c|}
\hline \multirow{2}{*}{$\begin{array}{l}\text { log2 fold } \\
\text { change }\end{array}$} & \multicolumn{2}{|c|}{ prdm14-GR vs. CC } \\
\cline { 2 - 3 } & Stage 14 & Stage 27 \\
\hline$\downarrow>5$ & 0 & 0 \\
\hline$\downarrow>3$ & 3 & 0 \\
\hline$\downarrow>2$ & 48 & 4 \\
\hline$\downarrow>1$ & $\mathbf{5 7 1}$ & $\mathbf{4 1}$ \\
\hline
\end{tabular}

Fig. 3.11 RNA-sequencing analysis of prdm14-GR overexpression in animal caps. (A-B) Prdm14-GR (500 pg) mRNA was injected into both blastomeres of two-cell stage embryos. Control and prdm14-GR mRNA-injected embryos were treated with dex from four-cell stage on and animal caps were isolated from blastula stage embryos. At the equivalent of neural plate stage (stage 14) and tailbud stage (stage 27) total RNA was isolated and analyzed by RNAsequencing. (B) Venn diagrams show the number of unique and shared target genes at stage 14 and 27, respectively. (C) Summary of identified putative target genes of Prdm14 after application of different fold-change categories. Given is the number of target genes with a log2 fold change higher than 1, 2, 3 or 5 compared to uninjected control caps (CC).

Applying these parameters, Prdm14 induced 338 genes at stage 14 and 356 genes at stage 27, respectively. From these a total of 65 genes were upregulated at both stages (Fig. 3.11B). Conversely, 571 and 41 genes were downregulated at stage 14 and 27, with only 11 genes shared between both stages. In a more stringent analysis using a minimum log2 fold change of three, prdm14-GR induced 68 and 208 genes at stage 14 and 27, respectively. In contrast the number of downregulated candidate genes dropped to three and zero at stage 14 and stage 27, respectively. For this reason, the further analysis 
was focused on the candidate genes upregulated by prdm14-GR overexpression.

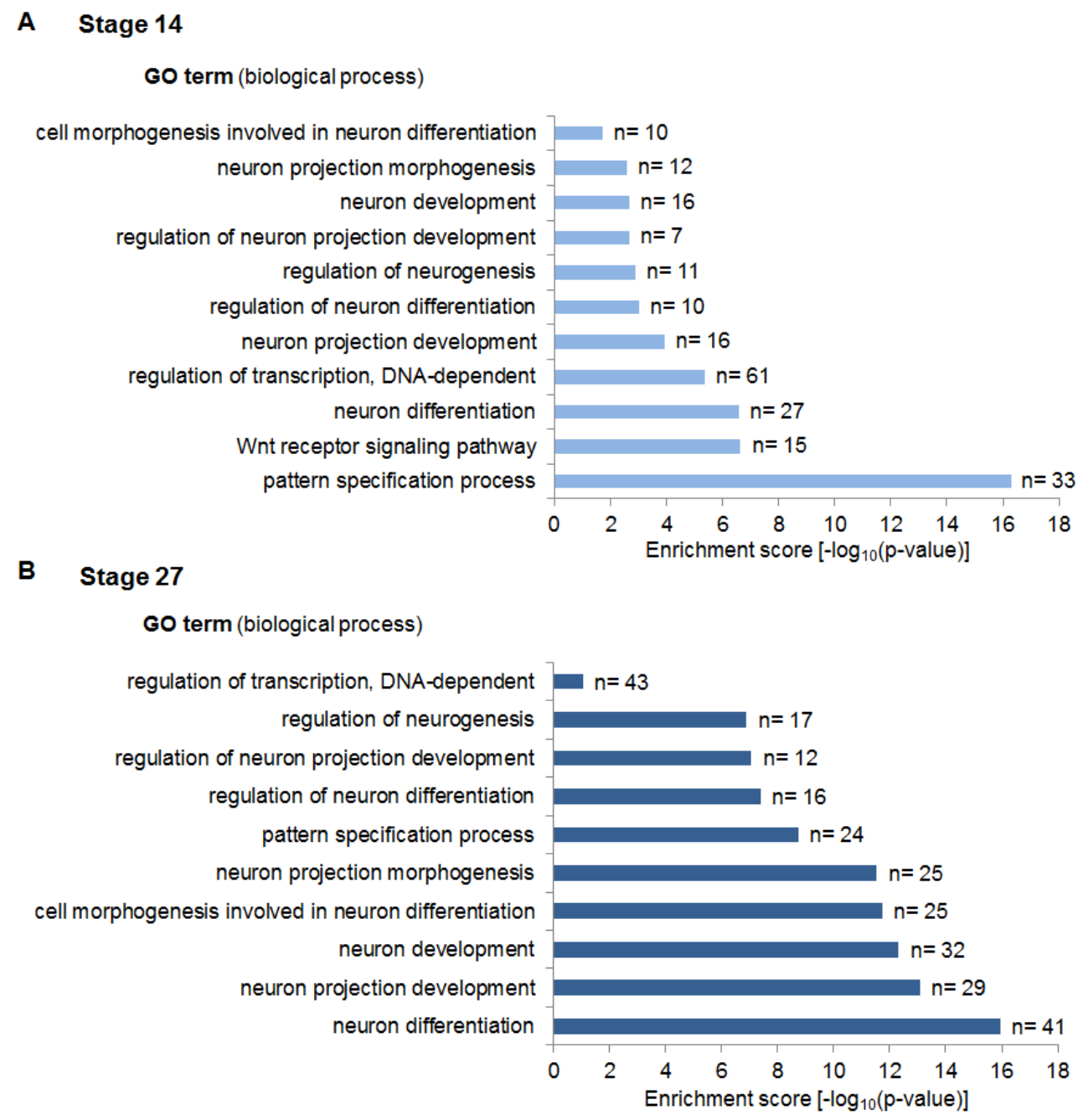

Fig. 3.12 Genes of biological processes involved in neural development are enriched in prdm14-GR overexpressing animal caps. Gene Ontology (GO) analysis was performed using DAVID (http://david.abcc.ncifcrf.gov) (Dennis et al. 2003). The number of genes in each category is shown as well as the $-\log _{10} \mathrm{P}$ value at false discovery rate.

Consistent with the previous results in embryos and in animal caps, the RNAsequencing analysis revealed a delayed neuronal differentiation induced by prdm14-GR, with the expression of tubb2b, t/x3 and slc17a7 all induced at the equivalent of stage 27 (Table 3.1). To gain insight into the pathways and processes of the Prdm14-induced genes, gene ontology (GO) analysis was performed (Appendix 6.2, Fig 3.12). Prdm14-GR upregulated genes at stage 14 were highly enriched in the category of pattern specification process, of which many were hox-transcription factors (hoxc6, hoxc8, hoxc9, hoxa5, hoxa7, hoxc5, hoxa10, hoxa9, hoxb4, hoxb7, hoxb8, hoxb6) (Fig. 3.12A). In addition, 
genes involved in the Wnt receptor signaling pathway, including multiple ligands, co-receptors and intracellular components (wnt8a, wnt10a, wnt10b, wnt5b, ryk, ror2, fzd7, fzd10) were also upregulated. Several processes, which are related to neural development, were upregulated at neural plate as well as tailbud stage, such as neuron differentiation, neuron projection development, morphogenesis or regulation of neurogenesis (Fig. 3.12B). Interestingly, among the strongly upregulated prdm14-GR genes were those involved in the process of neural crest formation such as wnt8, cdx1/2/4, pax3 and zic1 (Table 3.1).

\begin{tabular}{|c|c|c|c|}
\hline \multirow{2}{*}{ function } & \multirow{2}{*}{ Gene } & \multicolumn{2}{|c|}{ log2 fold change } \\
\hline & & Stage 14 & Stage 27 \\
\hline neural pre-patterning & zic3 & 8.23 & \\
\hline \multirow{2}{*}{ proneural } & neurog1 & 6.52 & \\
\hline & ebf2 & 4.27 & 4.96 \\
\hline glutamatergic selector gene & $t / x 3$ & 2.17 & 5.91 \\
\hline glutamate transporter & slc17a7 & & 5.78 \\
\hline postmitotic neurons & $t u b b 2 b$ & & 1.98 \\
\hline \multirow{5}{*}{ Wnt signaling } & $w n t 5 b$ & 1.48 & \\
\hline & wnt8a & 7.13 & \\
\hline & wnt10a & 5.39 & \\
\hline & axin2 & 4.05 & \\
\hline & ror2 & 4.55 & \\
\hline \multirow{5}{*}{ Neural crest induction } & fgf3 & 5.17 & \\
\hline & $c d \times 1$ & 8.98 & \\
\hline & $c d \times 2$ & 5.57 & 2.30 \\
\hline & $c d \times 4$ & 8.14 & 2.18 \\
\hline & sp5 & 7.21 & 2.06 \\
\hline \multirow{2}{*}{$\begin{array}{l}\text { Neural plate border } \\
\text { specification }\end{array}$} & pax3 & 5.51 & 4.82 \\
\hline & zic1 & 2.54 & \\
\hline \multirow{6}{*}{ Neural crest specification } & snai2 & & 1.92 \\
\hline & tfap $2 b$ & 2.81 & 2.57 \\
\hline & foxd3 & & 6.34 \\
\hline & sox9 & 2.09 & \\
\hline & sox10 & & 7.92 \\
\hline & prdm1a & 1.83 & \\
\hline
\end{tabular}

Table 3.1 List of selected candidate genes upregulated in prdm14-GR overexpressing animal caps. Overview of selected candidate genes with their log2 fold changes at stage 14 and 27 respectively. 


\subsection{Prdm14 activates canonical Wnt-signaling}

Many components of the Wnt-signaling pathway were identified to be upregulated by prdm14-GR in the RNA-sequencing analysis. As the Wnt pathway is upstream of many of the genes that were also induced, the activation of the Wnt pathway was subject to further verification.

Ligands associated with both the non-canonical and canonical Wntpathways were induced. Therefore, luciferase reporter assays were performed to measure the activation of these two different pathways. Atf2-luc and TOPflash served as reporters for non-canonical and canonical signaling, respectively (Korinek et al., 1997; Ohkawara and Niehrs, 2011). The reporters were injected together with prdm14-GR, wnt5a or wnt8a, which served as positive controls for the non-canonical and canonical reporters (Fig. 3.13A). At stage 14 embryonic lysates were prepared and luciferase activity quantified. Consistent with previous reports (Ohkawara and Niehrs, 2011), the atf2-luc reporter was strongly (12-fold) activated upon injection of wht5a but not of wnt8a mRNA. Conversely, the TOPFlash reporter was induced (2-fold) by wnt8a but not by wnt5a mRNA injection. Upon overexpression of prdm14-GR the non-canonical atf2-luc reporter was only sligthly activated compared to wnt5a (Fig. 3.13B). However, prdm14-GR induced the canonical TOPflash reporter to similar levels as those observed for wnt8a. The difference in the activation level of the non-canonical and canonical Wnt-pathways by Prdm14 is also reflected in the data obtained from the RNA-sequencing. There Prdm14GR activates wnt8a (log2FC, 7.1) to a much higher extent than wnt5b (log2FC, 1.5). Consistent with the results from the RNA-sequencing, Prdm14-GR activates wnt8 expression in whole embryos at stage $14(n=27 ; 78 \%$ increase $)$ (Fig. 3.13D, red arrows). The activation of the TOPFlash reporter by Prdm14GR further supports the notion that Prdm14 activates canonical Wnt-signaling in embryos at neural plate stage. 
A

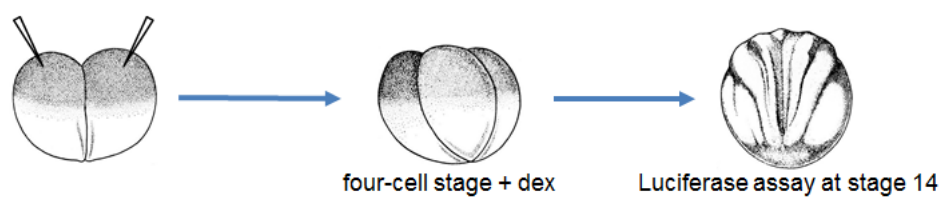

B
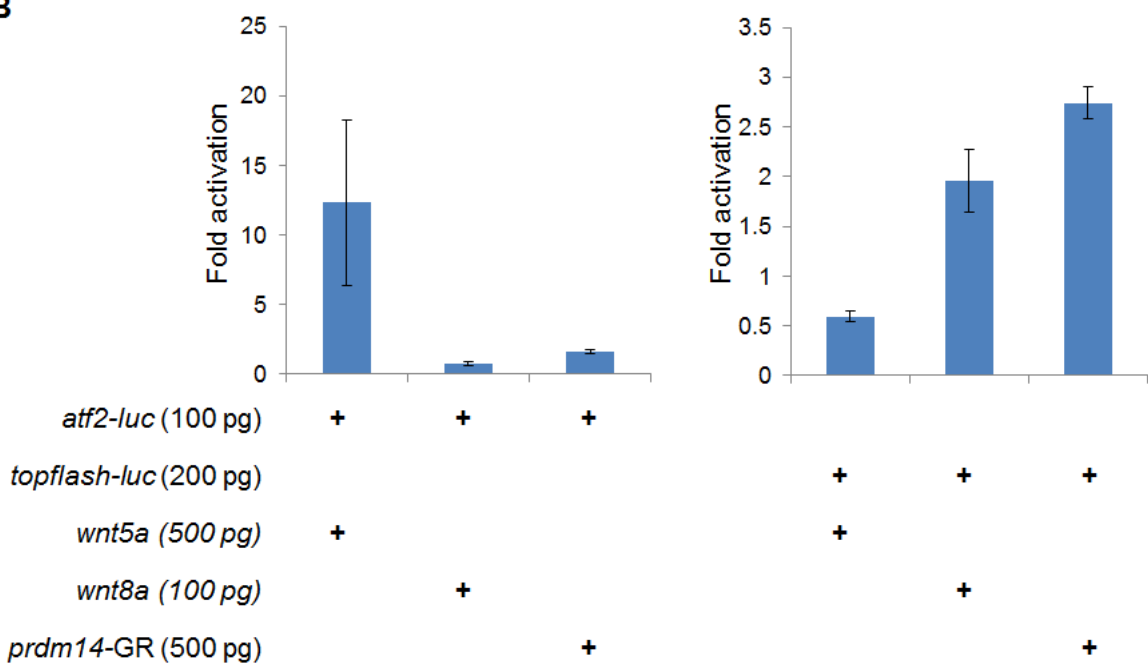

C
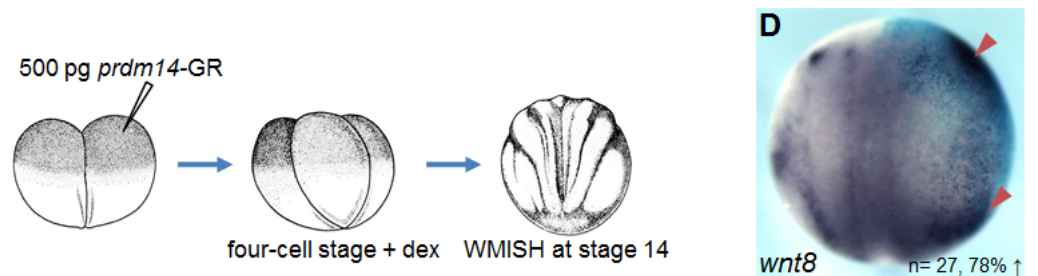

Fig. 3.13 Prdm14-GR overexpression activates canonical Wnt-signaling. (A) Atf2-luc and topflash-luc reporter were injected together with indicated mRNA into both blastomeres of twocell stage embryos. Embryos were cultivated until open neural plate stage and luciferase activity was measured and normalized to renilla luciferase. (B) Shown is a summary of three independent luciferase experiments. Six embryos per injection were collected in duplicates for each experiment. Error bars represent the standard error of the mean (+/-SEM). Note the different scales in each diagram. (C) Prdm14-GR (500 pg) mRNA together with $\beta-G a l$ (75 pg) mRNA (light blue staining) were injected into one blastomere of a two-cell stage embryo. Embryos were treated from four-cell stage on with dex. (D) Wnt8 expression was analyzed at stage 14 by whole mount in situ hybridization. The injected side is on the right, dorsal view, anterior up.

\subsection{Comparative expression analysis of prdm14 with neural plate border specifiers}

In addition to Wnt signaling components, the RNA-sequencing analysis suggests that prdm14-GR is sufficient to induce the expression of several genes involved in the gene regulatory network of neural crest formation. These include the upstream neural plate border specifiers pax3 and zic1 (Hong and SaintJeannet, 2007). To determine if prdm14 is present at the correct time and place to regulate pax3 and zic1, a comparative expression analysis was performed 
(Fig. 3.14). At stage 10.5 a broad expression of prdm14 in the dorsal ectoderm is observed, while at stage 11.5 the first strong and specific expression of pax3 and zic1 occurs. Pax3, zic1 and prdm14 are all expressed in the lateral neural plate border and the expression of zic1 and prdm14 extends into the anterior neural plate border until stage 13. At stage 14 zic1 is still expressed throughout the anterior neural plate border while anterior prdm14 expression is maintained in the trigeminal-profundal placode. During all developmental stages pax3 expression is restricted to the lateral neural plate border. Taken together, the observation that prdm14 expression precedes and also co-localizes with pax3 and zic1 expression, at the neural plate border, suggests an early function of prdm14 in the regulation of pax3 and zic1.
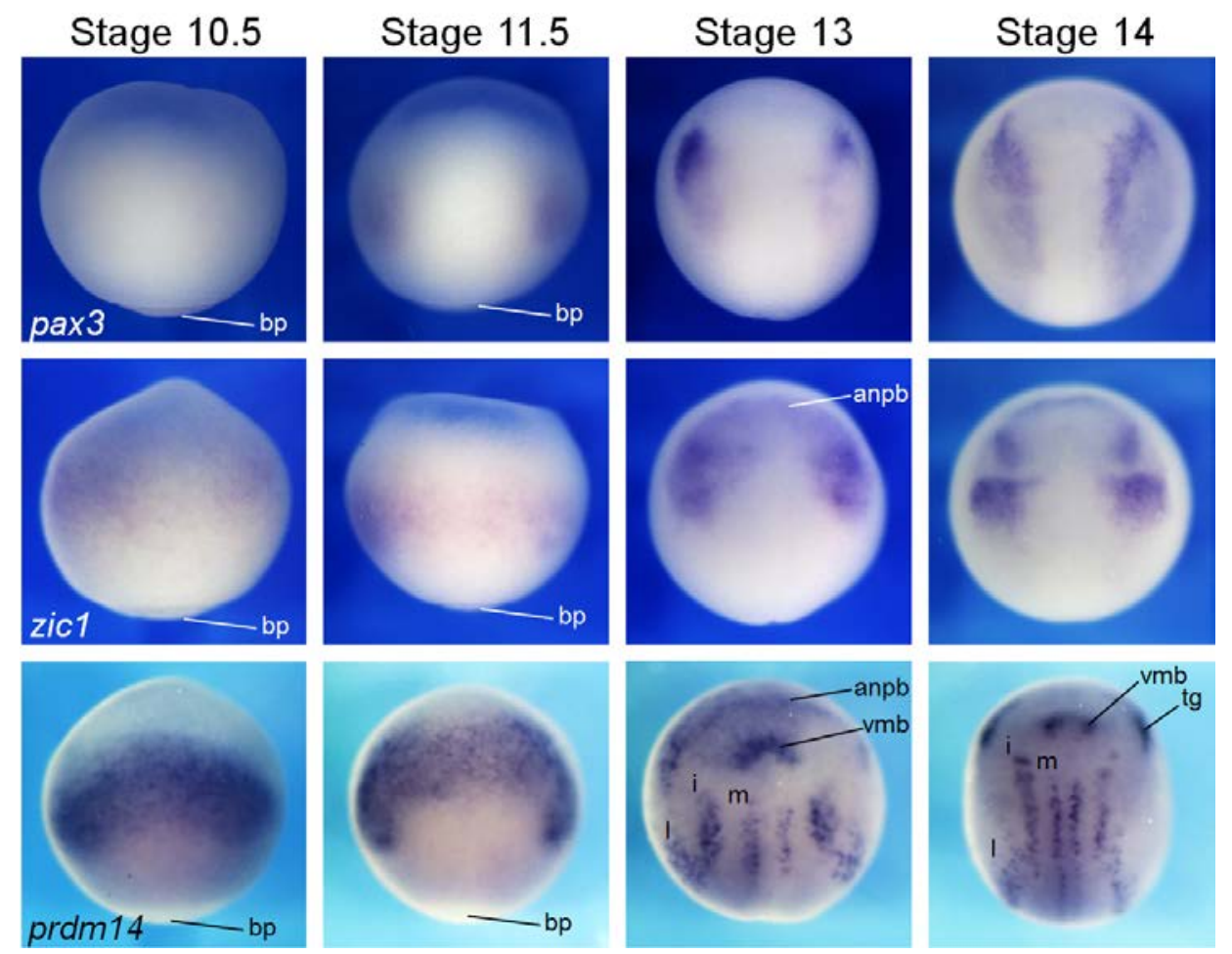

Fig. 3.14 Prdm14 is co-expressed with pax3 and zic1 in regions of the neural plate border. Comparative expression analysis of stage matched embryos by whole mount in situ hybridization using markers indicated on the bottom left. Stage 10.5-14: dorsal view, anterior up. anpb, anterior neural plate border; bp, blastoporus; i, intermediate; I, lateral; m, medial; tg, trigeminal-profundal placode; vmb, ventral midbrain 


\subsection{Knock down of Prdm14 has no influence on candidate gene expression}

To determine whether Prdm14 is required for the induction of selected prdm14-GR candidate genes (Table 3.1), their expression was evaluated under knock-down conditions. The specificity and efficiency of the SpMOs has been previously confirmed (Fig. 3.9). Therefore, both Prdm14 SpMOs were injected into one blastomere of two-cell stage embryos and marker gene expression was analyzed by whole mount in situ hybridization at stage 14 (Fig. 3.15A). While prdm14-GR overexpression results in an inhibition of tubb2b (Fig. 3.6B, F), Prdm14 loss of function showed no effect on $t u b b 2 b$ expression at neural plate stage (Fig. 3.15B'). The candidate genes wnt8, the neural plate border genes pax3, zic1, zic2 and zic3 as well as the neural crest specifying genes sox10 showed also no alteration in expression on the injected site (Fig. 3.15C-G, J). This was also the case for neurog1 and the Rohon-Beard sensory neuron marker $t / x 3$ (Fig. 3.15H-I). The fact that knock down of Prdm14 had no influence on the expression of the tested marker genes suggests that Prdm14 might not be necessary for their regulation. However, it cannot be excluded that residual Prdm14 is sufficient for its downstream activity or that redundancies exist with other Prdms. 

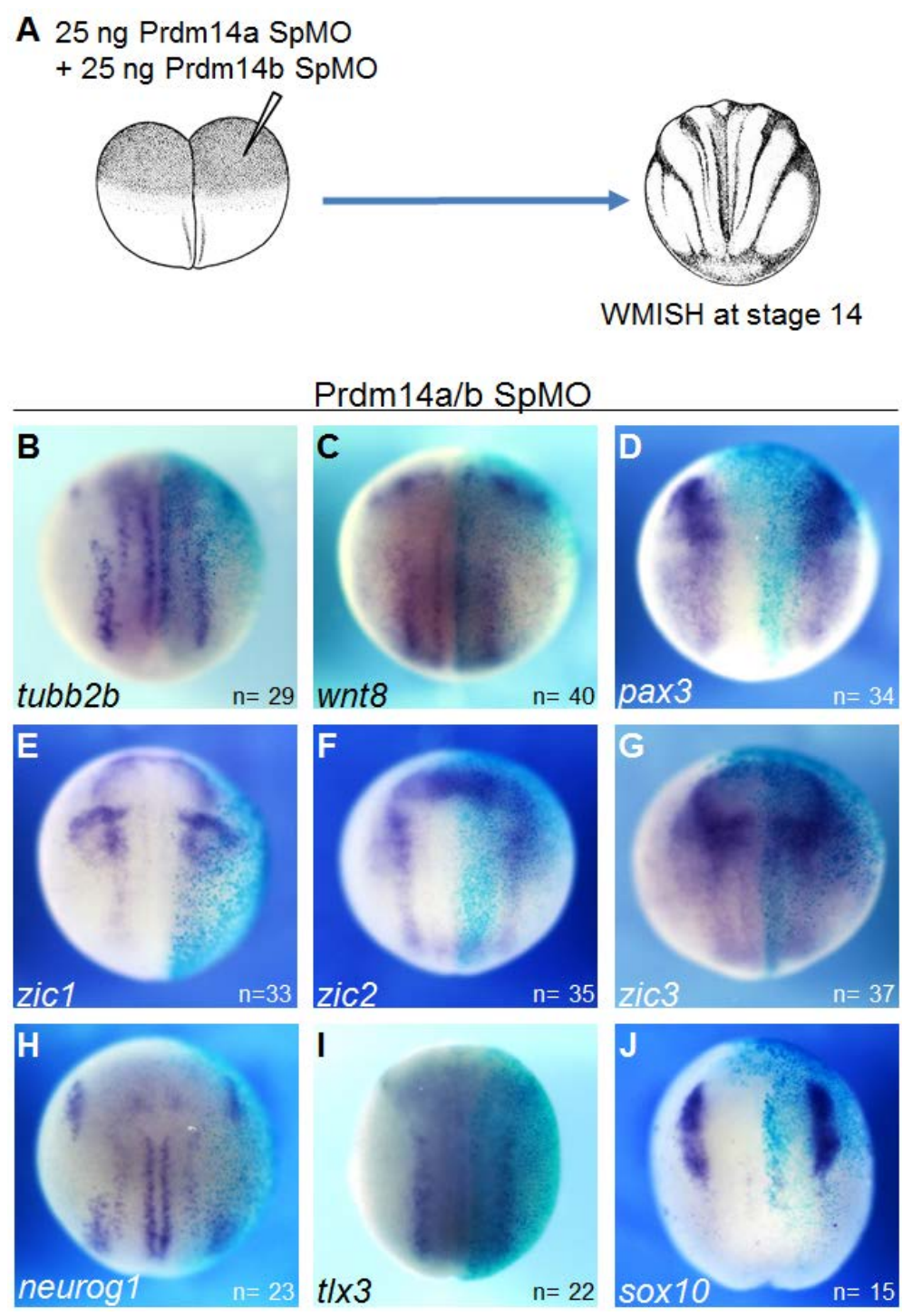

Fig. 3.15 Prdm14 knock down does not influence the expression of selected candidate genes in whole embryos. (A) Prdm14a SpMO and Prdm14b SpMO (25 pg each) together with $\beta$-Gal (75 pg) mRNA (light blue staining) were injected into one blastomere of a two-cell stage embryo. (B-J) Gene expression was analyzed at stage 14 by whole mount in situ hybridization using markers indicated on the bottom left. The injected side is on the right, dorsal view, anterior up.

\subsection{Prdm14 gain of function activates neural crest genes in whole embryos}

As the knock down of Prdm14 showed no effect on the expression of candidate genes, a gain of function analysis was performed to verify their regulation by Prdm14. Prdm14-GR mRNA was injected into one blastomere of two-cell stage embryos, which were treated with dexamethasone at the four-cell stage (Fig. 3.16A). 


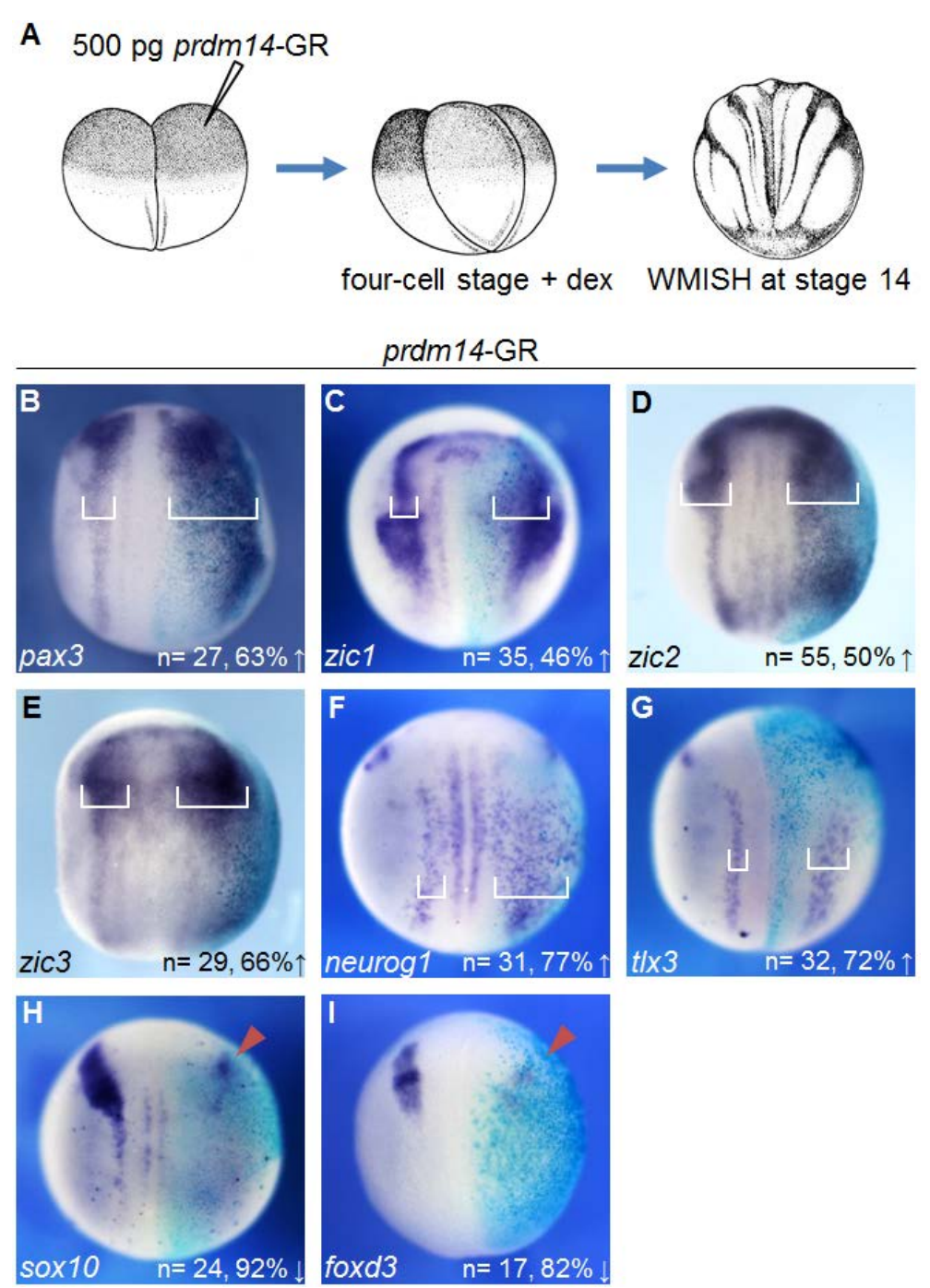

Fig. 3.16 Prdm14-GR overexpression promotes the expression of selected candidate genes in whole embryos. (A) Prdm14-GR (500 pg) mRNA together with $\beta$-Gal (75 pg) mRNA (light blue staining) were injected into one blastomere of two-cell stage embryos. Embryos were treated from four-cell stage on with dex. (B-I) Gene expression was analyzed at stage 14 by whole mount in situ hybridization using markers indicated on the bottom left. The injected side is on the right, dorsal view, anterior up. White brackets indicate width of expression domain.

As prdm14-GR overexpression activates a number of genes involved in the gene regulatory network of neural crest formation, the expression of the neural plate border specifiers pax3 and zic1 were analyzed (Fig. 3.16B-C). Prdm14-GR overexpression leads to the expansion of pax3 $(n=27 ; 63 \%$ increased) as well as zic1 ( $n=35 ; 46 \%$ increased) expression, although the effect on zic1 is much weaker (Fig. 3.16C). This observation is consistent with the RNA-sequencing data, which indicate a stronger activation of pax3 than of zic1 (Table 3.1). Interestingly, the neural plate border appeared enlarged on the injected side of the embryos. To verify this result, the expression of other neural plate border specific genes, which are members of the zic family namely zic2 
and zic3 was analyzed (Houtmeyers et al., 2013). These were also shown to be upregulated in the prdm14-GR overexpressing animal caps (Table 3.1). In agreement with previous observations, the expression of zic2 ( $n=55 ; 50 \%$ increased) and zic3 ( $n=29 ; 66 \%$ increased) was likewise expanded (Fig. 3.16DE). Beside genes involved in neural crest formation, the proneural transcription factor neurog1 was also highly upregulated in the prdm14-GR overexpressing animal caps (Table 3.1). Consistently, an expansion of neurog1 expression could be shown ( $n=31 ; 77 \%$ increased), whereas the expression in the trigeminal placode was alleviated (Fig. 3.16F).

The RNA-sequencing data revealed not only a strong activation of the glutamatergic sensory marker $t / x 3$ at the tailbud stage, but also a weak activation at neural plate stage (Table 3.1). This led to the question, wether prdm14 promotes the earliest step of Rohon-Beard sensory neuron differentiation at the neural plate stage. Indeed, on the injected side $t / x 3$ expression was expanded in the neural plate border ( $n=32 ; 72 \%$ increased), but inhibited in the trigeminal placode (Fig. 3.16G).

In addition, the RNA-sequencing data revealed a strong upregulation of the neural crest specifiers sox10 and foxd3 at tailbud stage (Table 3.1). These genes are already active at the neural plate stage (Pohl and Knöchel, 2001; Aoki et al., 2003), therefore the potential role of Prdm14-GR in the regulation of their expression was analyzed. Interestingly, these marker genes were inhibited at neural plate stage upon prdm14-GR overexpression (sox10: $n=24 ; 92 \%$ downregulated, red arrow; foxd3: $n=17 ; 82 \%$ downregulated, red arrow) (Fig. $3.16 \mathrm{H}-\mathrm{I})$. 
A $\quad 500$ pg prdm14-GR

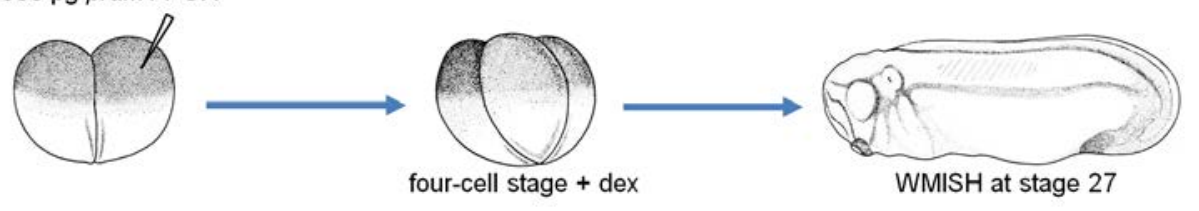

prdm14-GR
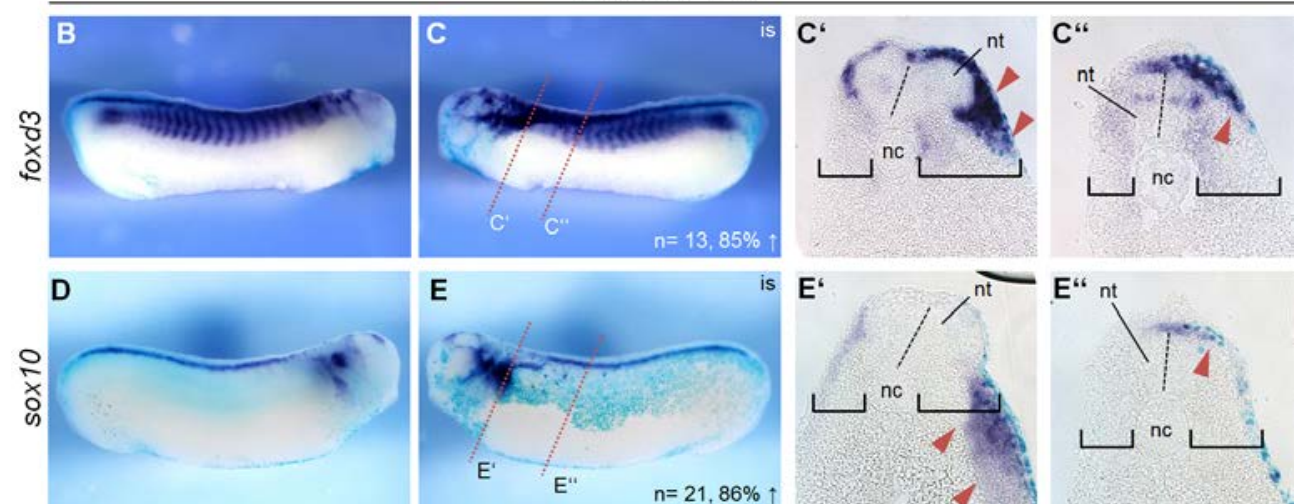

E

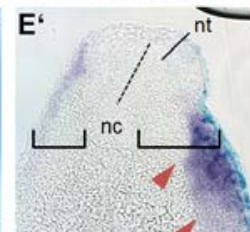

E“" nt
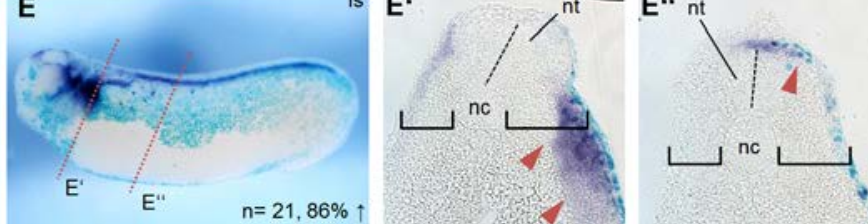

Fig. 3.17 Prdm14-GR overexpression promotes the neural crest formation in whole embryos. (A) Prdm14-GR (500 pg) mRNA together with $\beta$-Gal (75 pg) mRNA (light blue staining) were injected into one blastomere of two-cell stage embryos. Embryos were treated from four-cell stage on with dex. (B-E') Gene expression was analyzed at stage 27 by whole mount in situ hybridization using markers indicated on the left. The injected side is on the right, lateral view. is, injected side (C', C', E', E') Transverse sections at indicated levels of embryo shown in $C$ and $E$, respectively. Black brackets indicate width of mesenchymal tissue on the level of the notochord. Dashed line indicates midline of the neural tube. nt, neural tube; nc, notochord

However, consistent with the RNA-sequencing data, prdm14-GR microinjection led to an ectopic expression of sox10 and foxd3 in tailbud stage embryos (Fig. 3.17B-E'). In cranial neural crest cells, foxd3 and sox10 expression was increased (Fig. 3.17C', D'), whereas the trunk neural crest was expanded laterally. In all prdm14-GR injected embryos thickening of the mesenchymal tissue on the injected side could be observed (Fig. 3.17C', E'; black bracket). Consequently, these findings suggests that prdm14-GR leads to the expansion and activation of the expression of neural plate border genes, which subsequently might lead to the induction of downstream genes involved in neural crest specification such as sox10 and foxd3.

In summary this study demonstrated that prdm14 is expressed during gastrulation in the prospective neuroectoderm and later during development in the territories of primary neurogenesis. Consistently, overexpression of the proneural transcription factor Neurog2 ectopically activates prdm14 expression. At neural plate stage prdm14 promotes and maintains the proliferation of neural precursors and therefore delays differentiation at neural plate stage. 
Overexpression of prdm14 activates canonical Wnt-signaling, expands the neural plate border and promotes the expression of neural plate border genes. Consequently this might lead to the ectopic differentiation of neural plate border derivatives such as Rohon-Beard sensory neurons and neural crest cells. 


\section{Discussion}

Regionalized specification and differentiation of the ectoderm is under the control of multiple intrinsic and extrinsic factors. The prospective epidermal cells are characterized by high BMP signaling, while neural fate differentiation within the dorsal ectoderm requires low BMP signaling and the presence of FGF signaling (Rogers et al., 2009; Pera et al., 2014). In contrast, Wnt-signaling is required for posterior fates but must be inhibited for anterior neural fates (McGrew et al., 1997). The neural plate border separates the neural plate from the non-neural ectoderm and is established through the interaction of Wnt- and FGF-signaling, as well as intermediate levels of BMP signaling, and will give rise to the neural crest, the neurogenic placodes and Rohon-beard sensory neurons (Simoes-Costa and Bronner, 2015). In this thesis, the role of Prdm14, a member of the PR domain family of transcriptional regulators, in the context of ectodermal differentiation is described.

The expression of prdm14 in the prospective neuroectoderm is suggestive for a role in the establishment of the neural progenitor cells. In agreement, prdm14-GR promoted proliferation and expansion of the progenitor pool in the neural plate, while neuronal differentiation was delayed. Prdm14 was shown to positively regulate the canonical Wnt pathway and the key neural plate border specifying genes, pax3 and zic1. Correspondingly, Prdm14 was sufficient to promote derivatives of the neural plate border in pluripotent cells and embryos including neural crest cells and Rohon-Beard glutamatergic sensory neurons. Prdm14 was also positively regulated by the proneural transcription factor Neurog2 and expressed in postmitotic neurons suggesting an additional function in the context of the development of the nervous system.

\subsection{Prdm14 maintains the proliferation of precursor cells}

Previous studies have described a role for Prdm14 in the establishment of primordial germ cells (PGC) in mice. In this context, Prdm14 together with Prdm1 (Blimp1) promotes stemness characteristics through activation of the pluripotency gene sox2 (Yamaji et al., 2008). In hESCs, Prdm14 interacts with the core pluripotency genes by co-occupying target genes together with Sox2, 
Nanog and Oct4. During the development of the nervous system, sox2 serves a marker for multipotent proliferating neural progenitor cells and is expressed throughout the entire neural plate (Graham et al., 2003; Ellis et al., 2004). Similar to a role in ESCs, prdm14 overexpression in $X$. laevis embryos resulted in an expansion of sox2 expression and an increase in the number of $\mathrm{pH} 3$ positive cells, which marks mitotically active cells. This increase in proliferation occurred at the expense of differentiation, as shown by the transient inhibition of the post-mitotic neuronal marker tubb2b. The ability to promote proliferation and maintenance of a progenitor cell fate fits well with the strong and specific expression of prdm14 in the prospective neuroectoderm at the onset of gastrulation. Prdm14 is also present in the anterior neural plate at the end of gastrulation, which maintains its proliferative capacity and undergoes delayed neuronal differentiation (Hartenstein, 1989; Eagleson and Harris, 1990; Hartenstein, 1993; Papalopulu and Kintner, 1996).

In the RNA-sequencing analysis, sox2 was only weakly $(\log 2 \mathrm{FC}, 0.6)$ induced by prdm14 compared to control caps at the equivalent of neural plate stage. This suggests that prdm14 may not be sufficient to promote sox2 expression and requires the cooperation with other factors. Another possible explanation might be that the levels of sox 2 are already decreasing in prdm14GR overexpressing animal cap cells. Therefore, analysis of earlier time points might provide more insight in the transcript levels of sox2 in prdm14-GR overexpressing animal caps.

Intriguingly, RNA-sequencing analysis revealed that prdm14 overexpression in animal caps activated the expression of prdm1 at neural plate stage. This is of particular interest, as Prdm14 in combination with Prdm1 is known to activate the expression of sox2 during mouse PGC specification (Yamaji et al., 2008). However, it must be mentioned, that prdm14 expression in mice is specific for PGCs and has not been show in the central nervous system (Yabuta et al., 2006; Yamaji et al., 2008). Conversely, in X. laevis prdm14 expression cannot be detected in PGCs and is restricted to the developing nervous system. As the genomic locus with respect to flanking genes of prdm14 is the same in mice and $X$. laevis, the regulation of prdm14 expression must have changed during evolution. The lack of expression of prdm14 in $X$. laevis PGCs compared to mice may be attributed to the different mechanisms of PGC 
specification in both species. While mice PGCs are specified by signals from the surrounding somatic tissue, the specification of PGCs in $X$. laevis and zebrafish requires the inheritance of maternal germ plasm (Johnson et al., 2011). Therefore it is possible, that the interaction of Prdm14 and Prdm1 is conserved, although in a different developmental context. This is supported by the fact that in $X$. laevis and zebrafish prdm1 is also not expressed in PGCs, but in the neural plate border where it is required for Rohon-Beard sensory neurons (de Souza et al., 1999; Rossi et al., 2009; Klymkowsky et al., 2010). Furthermore, $X$. laevis prdm1 plays a role in the anterior endomesoderm, where it is required for head induction (de Souza et al., 1999).

As the overexpression of prdm14 influences the expression of the neural plate marker sox2 in $X$. laevis, Prdm14 might be involved in the establishment of the neuroectoderm. Furthermore, overexpression of prdm14 in animal caps induced the expression of components of the FGF signaling pathway (fgf3, fgfr1), whose activity is required for neural induction (Kengaku and Okamoto, 1995; Lamb and Harland, 1995; Streit et al., 2000; Pera et al., 2003; Linker and Stern, 2004, Delaune et al., 2005). In summary, the maintained proliferation upon prdm14 overexpression is in agreement with the known function of prdm14 to promote and maintain the stemness character in mESC and hESC (Tsuneyoshi et al., 2008, Chia et al., 2010; Ma et al., 2011)

\subsection{Prdm14 activates canonical Wnt-signaling in $X$. laevis}

The balance between stemness and differentiation in adult stem cell niches requires the activity of canonical Wnt-signaling (Fodde and Brabletz, 2007; Holland et al., 2013). Consequently, the deregulation of canonical Wntsignaling is a common occurrence in the formation of cancer. A Wnt-pathway modulating activity has been described so far only for Prdm5 (Meani et al., 2009; Shu et al., 2011). It has been shown that Prdm5 functions as a tumor suppressor in part through down regulation of the canonical Wnt-signaling pathway (Meani et al., 2009; Shu et al., 2011). Interestingly, overexpression of prdm14 activated several components of the Wnt-pathway in $X$. laevis animal caps. Additionally, as demonstrated by the TOPFlash luciferase reporter, prdm14 led to an increase in canonical Wnt-signaling. 
Prdm14 has also been described to play a role in carcinogenesis, where it was first described to promote the proliferation of breast cancer cells (Nishikawa et al., 2007). Furthermore, an involvement of Prdm14 in other cancer types like lymphoblastic lymphoma, lung cancer, testicular cancer and HPV-positive cancers was shown (Dettman and Justice, 2008; Simko et al., 2012; Zhang et al., 2013; Ruark et al., 2013; Snellenberg et al., 2014; Hubers et al., 2015). Interestingly, in $X$. laevis embryos and animal caps the overexpression of prdm14 promotes the expression of the glutamatergic sensory marker $t / x 3$, which has also been described to be upregulated in pediatric T-cell acute lymphoblastic leukemia (T-ALL) (Sayitoğlu et al., 2012; Moussa and Sidhom, 2013). This type of cancer is also characterized by elevated levels of prdm14 (Dettman et al., 2011; Simko et al., 2012; Carofino et al., 2013). Therefore, it might be of interest to study if and how these genes interact in the formation of T-ALL in humans.

Although prdm14 is deregulated in many types of cancer and its overexpression activates canonical Wnt-signaling in $X$. laevis, no connection between the deregulation of Wnt-signaling and Prdm14 in the context of carcinogenesis has been shown thus far. However, consistent with the observation that Prdm14 activates canonical Wnt-signaling, several downstream targets were activated such as the glutamatergic sensory marker t/x3, posterior neural plate markers $c d x 1 / 2 / 5$ and the neural plate border specifiers pax3 and zic1.

\subsection{Prdm14 activates the expression of neural plate border genes}

The neural plate border (NPB) is the source for multiple derivatives such as the neurogenic placodes, Rohon-Beard sensory neurons and neural crest cells (Hong and Saint-Jeannet, 2007; Rossi et al., 2009). The establishment of the NPB requires FGF-, Notch-, Wnt- signaling and intermediate levels of BMP signaling (Pegoraro and Monsoro-Burq, 2012; Simões-Costa and Bronner, 2015). The activity of these different signaling pathways induces the expression of neural plate border specifier genes such as $m s x 1$, dlx3, pax3 and zic1. Pax3 and zic1 in combination are sufficient to induce the expression of neural crest specifying genes such as sox8/9/10, snai2 and foxd3 (Milet et al., 2013; 
Plouhinec et al., 2014). Recently, large scale screening approaches could identify several pax3 and zic1 target genes, which might be involved in the formation of the developing neural crest (Plouhinec et al., 2014; Bae et al., 2014).

Interestingly, prdm14 overexpression led to the expansion of the neural plate border and induced the expression of the neural plate border specifying genes pax3 and zic1. In $X$. laevis, the regulation of pax3 and zic1 requires canonical Wnt-signaling (Bang et al., 1999, Sato et al., 2005). As previously mentioned, prdm14 overexpression in neural plate stage animal caps activated canonical Wnt-signaling and downstream targets such as $c d x 1 / 2 / 4$ and $s p 5$, which are known to be required for Wnt-mediated pax3 expression (SanchezFerras et al., 2012; Park et al., 2013). Hence, prdm14 might activate canonical Wnt-signaling, which in turn, activates pax3 through the action of $c d \times 1 / 2 / 4$ and sp5. The observation that pax3 was much stronger activated than zic1 in prdm14 overexpressing animal caps might be the result of a positive autoregulation of pax3 (Plouhinec et al., 2014). It is of interest that other neural plate border specifying genes such as $m s x 1$ and $d l x 3$ were not found to be upregulated in prdm14-GR overexpressing animal caps. Recently described

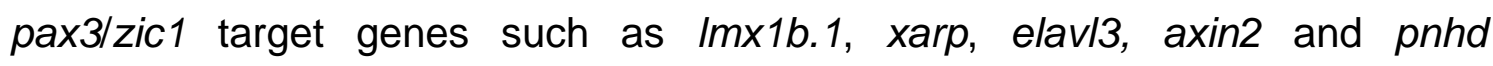
(Plouhinec et al., 2014) have also been identified as prdm14 downstream targets in the animal cap assay, supporting the notion that prdm14 activates pax3 and zic1 expression.

Consistent with the early activity of prdm14 to promote proliferation and to maintain a progenitor cell fate, the neural crest specifying genes sox10 and foxd3 were inhibited at neural plate stage in prdm14-GR mRNA microinjected embryos. However, this inhibition was only transient as tailbud stage embryos showed ectopic expression of sox10 and foxd3 in the cranial neural crest. It remains to be addressed, if the ectopic expression at tailbud stage is due to an increased pool of progenitors or due to the increased expression of pax3 and zic1.

It is known that pax3 and zic1 are sufficient to activate the gene regulatory network for neural crest formation (Hong and Saint-Jeannet, 2007; Plouhinec et al., 2014). Therefore, the induced expression of neural crest specifying genes such as snai2, foxd3, sox9/10 and tfap2b/e in prdm14-GR 
overexpressing animal caps may be the consequence of pax3 and zic1 activation. These findings support a role for prdm14 in the early establishment of the neural plate border. However, it will be of interest to test how other neural crest genes, which are not activated in prdm14-GR overexpressing animal caps, behave upon prdm14-GR overexpression.

\subsection{Prdm14 gain of function phenocopies zic1/2/3 overexpression}

Overexpression of the neural plate border genes zic1, 2 and 3 in $X$. laevis promotes neural crest formation and causes an expansion of the neural plate (Nakata et al., 1997, Nakata et al., 1998). At tailbud stage an enlargement of the neural tube and mesenchymal tissue on the injected side is observed when zic1, 2 and 3 are overexpressed. Interestingly, the expansion of the neural plate as well as the enlargement of the neural tube and mesenchymal tissue can also be detected upon ectopic prdm14 expression in whole embryos. Consistently, in prdm14 overexpressing animal caps and embryos the expression of zic1, zic2 and zic3 is increased. Therefore, it is possible that the prdm14-induced expansion of sox2 expression, as well as the enlargement of the neural tube and mesenchymal tissue, are the result of zic activation. Zic2 might be responsible for the inhibition of $t u b b 2 b$ at neural plate stage as it inhibits neuronal differentiation (Brewster et al., 1998) and is also activated by prdm14-GR.

Zic genes are not only described as activators (Merzdorf and Sive, 2006), but also as inhibitors of canonical Wnt-signalling (Fujimi et al., 2012; Pourebrahim et al., 2011), which would conflict with the finding that Prdm14-GR activates canonical Wnt-signaling. Further analyses will clarify, whether the activation of wnt8a by Prdm14-GR is required for its downstream activity and if the activation of wnt8a is direct or indirect.

\subsection{Prdm14 promotes sensory neuron formation}

The expression of prdm14 in the prospective neuroectoderm suggests an early function in the establishment of the neural plate. In addition to this early role, the expression of prdm14 in the territories of primary neurogenesis and in 
the postmitotic neurons in the neural tube is indicative for a later role in neuronal subtype differentiation and maturation. This notion is further supported by the positive regulation of prdm14 by Neurog2.

Recently, it has been shown that Prdm12 is required for the specification of $X$. laevis sensory neurons (Nagy et al., 2015). Overexpression of prdm12 induces the expression of transcription factors required for sensory neuron specification such as brn3d (pou4f1.2), sncg, drgx and t/x3. Interestingly, overexpression of prdm14 activates the same set of genes in animal caps, suggesting that Prdm14 might also be involved in the specification of sensory neurons. This idea is further supported by the finding that prdm14 overexpression induces ectopic neuronal cells that express markers indicative for glutamatergic sensory neurons.

Glutamatergic Rohon-Beard sensory neurons are the first to be born and arise from the neural plate border (Hernandez-Lagunas et al., 2005; Rossi et al., 2009; Olesnicky et al., 2010). Well-characterized transcription factors involved in the specification of this class of neurons are prdm1, neurog1 and neurog2, which upon overexpression induce ectopic sensory neuron formation (Olson et al., 1998; Perez et al., 1999; Perron et al., 1999; Cornell and Eisen, 2002; Hernandez-Lagunas et al., 2005). Interestingly, the expression of these transcription factors was activated by Prdm14, indicating an upstream role of Prdm14 on sensory neuron formation. The observation that prdm14 precedes the expression of neurog1 suggests that Prdm14 may be a direct regulator of neurog1.

The specification of sensory neurons has also been linked to canonical Wnt-signaling. Kondo and colleagues showed, that neuralized mesenchymal stem cells are able to differentiate into neurons in the presence of canonical Wnt-signaling. Interestingly, these differentiating cells express neurog 1 and $t / x 3$ (Kondo et al., 2011). As prdm14 activates canonical Wnt-signaling as well as the expression of neurog 1 and $t / x 3$, there is the intriguing possibility that canonical Wnt-signaling is involved in the process of sensory neuron specification in $X$. laevis.

Similar to $X$. laevis, prdm14 is expressed in zebrafish throughout the anterior neural plate at bud stage (Liu et al., 2012). At the 3-somite stage, prdm14 is co-expressed with neurog1 in dorsal and ventral neuron precursors 
and at the 26-somite stage in Rohon-Beard sensory and motor neurons. Prdm14 knock down in zebrafish does not influence neuronal differentiation (Liu et al., 2012). However, shortened axon outgrowth of CaP motor neurons can be observed at $26 \mathrm{hpf}$, which leads to impaired touch response and swimming behavior. This is only a transient effect as axon outgrowth is restored in embryos at $2.5 \mathrm{dpf}$, which was attributed to residual Prdm14 activity.

In this study, functionally verified splicing morpholinos for prdm14a and prdm14b were used to knock down both prdm14 paralogs. Similar to zebrafish, the knock down of Prdm14 in $X$. laevis embryos had no effect on neuronal differentiation. In addition, the expression of the selected candidate genes, identified in the RNA-sequencing analysis of prdm14-GR overexpressing animal caps, was not affected. Furthermore, the motor neuron axon outgrowth was not affected in tailbud stage $X$. laevis embryos after Prdm14 knock down suggesting that prdm14 is not necessary in these contexts. As Prdm14 is maternally supplied, it cannot be ruled out that the use of the splicing morpholinos is not effective enough and that the residual Prdm14 proteins might be sufficient to exert their function. A further explanation might be the existence of functional redundancies, as described in zebrafish for prdm3, 5 and 16 (Ding et al., 2013). This is likely, considering that all members of the prdm family are expressed in the developing central nervous system of $X$. laevis (Eguchi et al., 2015). A potential candidate might be prdm12, which also promotes the specification of sensory neurons and therefore might compensate the Prdm14 knock down. To address the question, if redundancies might exist between different Prdms, it would be of interest to knock down more than one Prdm or use gene editing tools like TALENs or CRISPR/Cas to efficiently knock out prdm14. 


\section{Conclusion}

In summary, this study suggests a role for the PR domain protein Prdm14 in the establishment of the neuroectoderm and maintenance of a proliferative state. As a consequence the pool of progenitor cells is expanded in the neural plate and neural plate border at the expense of differentiation. Since Prdm14 is able to induce the expression of zic1, 2 and 3, this expansion may also occur due to their activity. Through the prdm14-mediated activation of canonical Wnt-signaling the neural plate border specifying genes pax3 and zic1 are induced, which promote the formation of neural crest. The expansion of the neural plate border might also play a role in this context. The ectopic specification of Rohon-Beard sensory neurons could be caused by the expansion of the neural plate border or the activation of neurog1 by Prdm14. However, if the promotion of neural plate border derivatives is the direct consequence of an expanded progenitor pool or requires the activity of other factors remains to be further addressed.

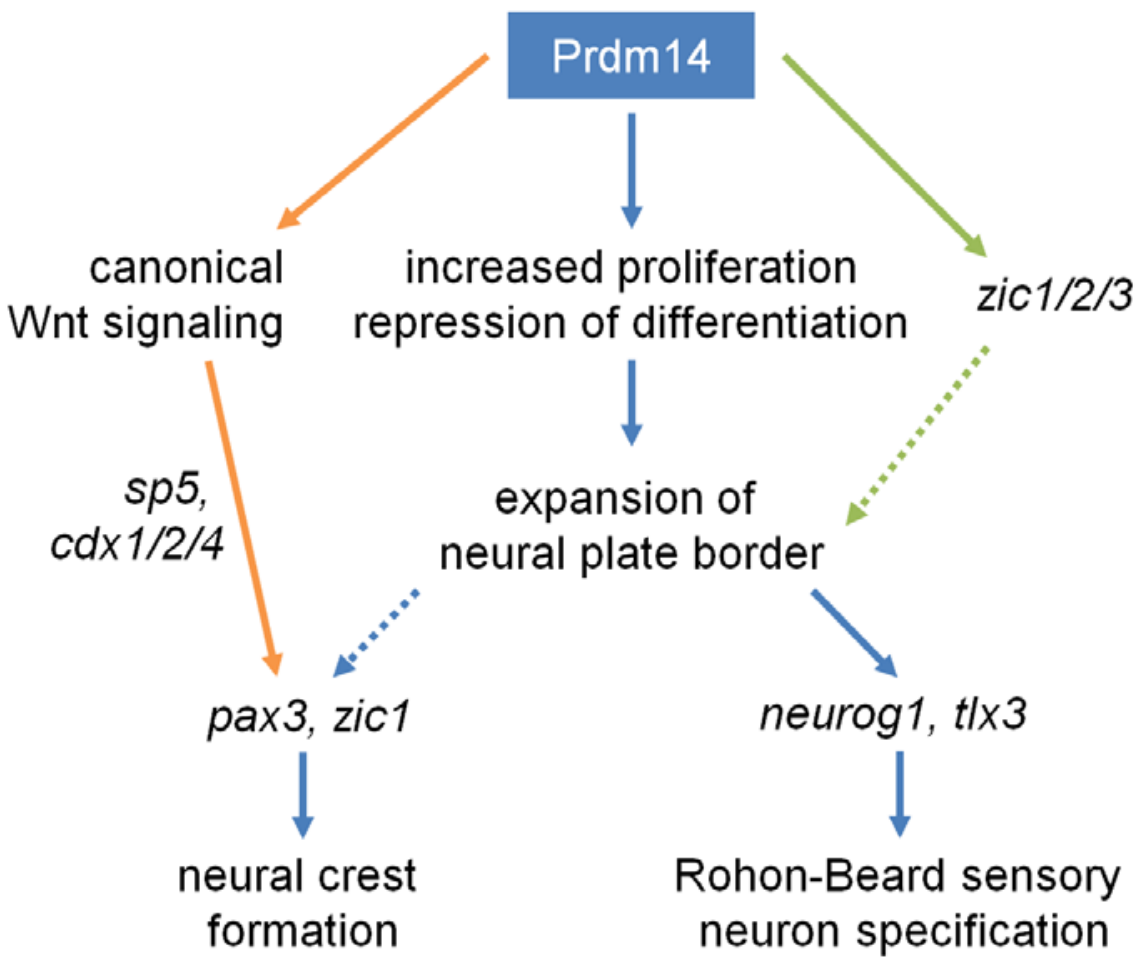

Fig. 5.1 Model for the function of Prdm14 in X. laevis. Prdm14 promotes a proliferative, undifferentiated state of progenitor cells. Furthermore, the expression of the zic genes is activated by Prdm14. Both processes might lead to the expansion of the neural plate border. Additionally, Prdm14 activates canonical Wnt-signaling which induces pax3/zic1 and promotes neural crest formation. The ectopic differentiation of Rohon-Beard sensory neurons is likely due to the expansion of the neural plate border or the induction of neurog1 by Prdm14. 


\section{Bibliography}

Alaynick, W. A., T. M. Jessell, and S. L. Pfaff. 2011. SnapShot: spinal cord development. Cell 146: 178-178 e171.

Amaya, E. 2005. Xenomics. Genome research 15: 1683-1691.

Anders, S., and W. Huber. 2010. Differential expression analysis for sequence count data. Genome biology 11: R106.

Aoki, Y., N. Saint-Germain, M. Gyda, E. Magner-Fink, Y. H. Lee, C. Credidio, and J. P. Saint-Jeannet. 2003. Sox10 regulates the development of neural crest-derived melanocytes in Xenopus. Developmental biology 259: 19-33.

Ariizumi, T., S. Takahashi, T. C. Chan, Y. Ito, T. Michiue, and M. Asashima. 2009. Isolation and differentiation of Xenopus animal cap cells. Current protocols in stem cell biology Chapter 1: Unit 1D 5.

Aruga, J., and K. Mikoshiba. 2011. Role of BMP, FGF, calcium signaling, and Zic proteins in vertebrate neuroectodermal differentiation. Neurochemical research 36: 1286-1292.

Bae, C. J., B. Y. Park, Y. H. Lee, J. W. Tobias, C. S. Hong, and J. P. SaintJeannet. 2014. Identification of Pax3 and Zic1 targets in the developing neural crest. Developmental biology 386: 473-483.

Bang, A. G., N. Papalopulu, M. D. Goulding, and C. Kintner. 1999. Expression of Pax-3 in the lateral neural plate is dependent on a Wntmediated signal from posterior nonaxial mesoderm. Developmental biology 212: 366-380.

Bard-Chapeau, E. A., J. Jeyakani, C. H. Kok, J. Muller, B. Q. Chua, J. Gunaratne, A. Batagov, P. Jenjaroenpun, V. A. Kuznetsov, C. L. Wei, R. J. D'Andrea, G. Bourque, N. A. Jenkins, and N. G. Copeland. 2012. Ecotopic viral integration site 1 (EVI1) regulates multiple cellular processes important for cancer and is a synergistic partner for FOS protein in invasive tumors. Proceedings of the National Academy of Sciences of the United States of America 109: 2168-2173.

Baudat, F., J. Buard, C. Grey, A. Fledel-Alon, C. Ober, M. Przeworski, G. Coop, and B. de Massy. 2010. PRDM9 is a major determinant of meiotic recombination hotspots in humans and mice. Science 327: 836840.

Beatus, P., and U. Lendahl. 1998. Notch and neurogenesis. Journal of neuroscience research 54: 125-136.

Bellefroid, E. J., C. Bourguignon, T. Hollemann, Q. Ma, D. J. Anderson, C. Kintner, and T. Pieler. 1996. X-MyT1, a Xenopus C2HC-type zinc finger protein with a regulatory function in neuronal differentiation. Cell 87: 1191-1202.

Bellmeyer, A., J. Krase, J. Lindgren, and C. LaBonne. 2003. The protooncogene c-myc is an essential regulator of neural crest formation in xenopus. Developmental cell 4: 827-839.

Bertrand, N., D. S. Castro, and F. Guillemot. 2002. Proneural genes and the specification of neural cell types. Nature reviews. Neuroscience 3: 517530.

Bhat, N., H. J. Kwon, and B. B. Riley. 2013. A gene network that coordinates preplacodal competence and neural crest specification in zebrafish. Developmental biology 373: 107-117. 
Bonev, B., P. Stanley, and N. Papalopulu. 2012. MicroRNA-9 Modulates Hes1 ultradian oscillations by forming a double-negative feedback loop. Cell reports 2: 10-18.

Borchers, A., and T. Pieler. 2010. Programming pluripotent precursor cells derived from Xenopus embryos to generate specific tissues and organs. Genes 1: 413-426.

Bouwmeester, T., S. Kim, Y. Sasai, B. Lu, and E. M. De Robertis. 1996. Cerberus is a head-inducing secreted factor expressed in the anterior endoderm of Spemann's organizer. Nature 382: 595-601.

Boy, S., J. Souopgui, M. A. Amato, M. Wegnez, T. Pieler, and M. Perron. 2004. XSEB4R, a novel RNA-binding protein involved in retinal cell differentiation downstream of bHLH proneural genes. Development 131: 851-862.

Boyer, L. A., T. I. Lee, M. F. Cole, S. E. Johnstone, S. S. Levine, J. P. Zucker, M. G. Guenther, R. M. Kumar, H. L. Murray, R. G. Jenner, D. K. Gifford, D. A. Melton, R. Jaenisch, and R. A. Young. 2005. Core transcriptional regulatory circuitry in human embryonic stem cells. Cell 122: 947-956.

Bray, S. J. 2006. Notch signalling: a simple pathway becomes complex. Nature reviews. Molecular cell biology 7: 678-689.

Brewster, R., J. Lee, and A. Ruiz i Altaba. 1998. Gli/Zic factors pattern the neural plate by defining domains of cell differentiation. Nature 393: 579583.

Burton, A., J. Muller, S. Tu, P. Padilla-Longoria, E. Guccione, and M. E. Torres-Padilla. 2013. Single-cell profiling of epigenetic modifiers identifies PRDM14 as an inducer of cell fate in the mammalian embryo. Cell reports 5: 687-701.

Carofino, B. L., B. Ayanga, and M. J. Justice. 2013. A mouse model for inducible overexpression of Prdm14 results in rapid-onset and highly penetrant T-cell acute lymphoblastic leukemia (T-ALL). Disease models \& mechanisms 6: 1494-1506.

Cau, E., S. Casarosa, and F. Guillemot. 2002. Mash1 and Ngn1 control distinct steps of determination and differentiation in the olfactory sensory neuron lineage. Development 129: 1871-1880.

Chalmers, A. D., D. Welchman, and N. Papalopulu. 2002. Intrinsic differences between the superficial and deep layers of the Xenopus ectoderm control primary neuronal differentiation. Developmental cell 2: 171-182.

Chan, Y. S., J. Goke, X. Lu, N. Venkatesan, B. Feng, I. H. Su, and H. H. Ng. 2013. A PRC2-dependent repressive role of PRDM14 in human embryonic stem cells and induced pluripotent stem cell reprogramming. Stem cells (Dayton, Ohio) 31: 682-692.

Chang, J. C., D. M. Meredith, P. R. Mayer, M. D. Borromeo, H. C. Lai, Y. H. Ou, and J. E. Johnson. 2013. Prdm13 mediates the balance of inhibitory and excitatory neurons in somatosensory circuits. Developmental cell 25: 182-195.

Cheung, M., M. C. Chaboissier, A. Mynett, E. Hirst, A. Schedl, and J. Briscoe. 2005. The transcriptional control of trunk neural crest induction, survival, and delamination. Developmental cell 8: 179-192.

Chia, N. Y., Y. S. Chan, B. Feng, X. Lu, Y. L. Orlov, D. Moreau, P. Kumar, L. Yang, J. Jiang, M. S. Lau, M. Huss, B. S. Soh, P. Kraus, P. Li, T. 
Lufkin, B. Lim, N. D. Clarke, F. Bard, and H. H. Ng. 2010. A genomewide RNAi screen reveals determinants of human embryonic stem cell identity. Nature 468: 316-320.

Chitnis, A., D. Henrique, J. Lewis, D. Ish-Horowicz, and C. Kintner. 1995. Primary neurogenesis in Xenopus embryos regulated by a homologue of the Drosophila neurogenic gene Delta. Nature 375: 761-766.

Chitnis, A. and C. Kintner. 1996. Sensitivity of proneural genes to lateral inhibition affects the pattern of primary neurons in Xenopus embryos. Development 122, 2295-301.

Christian, J. L., and R. T. Moon. 1993. Interactions between Xwnt-8 and Spemann organizer signaling pathways generate dorsoventral pattern in the embryonic mesoderm of Xenopus. Genes \& development 7: 13-28.

Coffman, C. R., P. Skoglund, W. A. Harris, and C. R. Kintner. 1993. Expression of an extracellular deletion of Xotch diverts cell fate in Xenopus embryos. Cell 73: 659-671.

Coles, E. G., L. A. Taneyhill, and M. Bronner-Fraser. 2007. A critical role for Cadherin6B in regulating avian neural crest emigration. Developmental biology 312: 533-544.

Cornell, R. A., and J. S. Eisen. 2002. Delta/Notch signaling promotes formation of zebrafish neural crest by repressing Neurogenin 1 function. Development 129: 2639-2648.

Couly, G., A. Grapin-Botton, P. Coltey, B. Ruhin, and N. M. Le Douarin. 1998. Determination of the identity of the derivatives of the cephalic neural crest: incompatibility between Hox gene expression and lower jaw development. Development 125: 3445-3459.

Creazzo, T. L., R. E. Godt, L. Leatherbury, S. J. Conway, and M. L. Kirby. 1998. Role of cardiac neural crest cells in cardiovascular development. Annual review of physiology 60: 267-286.

Damianitsch, K., J. Melchert, and T. Pieler. 2009. XsFRP5 modulates endodermal organogenesis in Xenopus laevis. Developmental biology 329: 327-337.

Dawson, S. R., D. L. Turner, H. Weintraub, and S. M. Parkhurst. 1995. Specificity for the hairy/enhancer of split basic helix-loop-helix (bHLH) proteins maps outside the bHLH domain and suggests two separable modes of transcriptional repression. Molecular and cellular biology 15: 6923-6931.

de Croze, N., F. Maczkowiak, and A. H. Monsoro-Burq. 2011. Reiterative AP2a activity controls sequential steps in the neural crest gene regulatory network. Proceedings of the National Academy of Sciences of the United States of America 108: 155-160.

de la Calle-Mustienes, E., A. Glavic, J. Modolell, and J. L. GomezSkarmeta. 2002. Xiro homeoproteins coordinate cell cycle exit and primary neuron formation by upregulating neuronal-fate repressors and downregulating the cell-cycle inhibitor XGadd45-gamma. Mechanisms of development 119: 69-80.

de Souza, F. S., V. Gawantka, A. P. Gomez, H. Delius, S. L. Ang, and C. Niehrs. 1999. The zinc finger gene Xblimp1 controls anterior endomesodermal cell fate in Spemann's organizer. The EMBO journal 18: $6062-6072$. 
Delaune, E., P. Lemaire, and L. Kodjabachian. 2005. Neural induction in Xenopus requires early FGF signalling in addition to BMP inhibition. Development 132: 299-310.

Delwel, R., T. Funabiki, B. L. Kreider, K. Morishita, and J. N. Ihle. 1993. Four of the seven zinc fingers of the Evi-1 myeloid-transforming gene are required for sequence-specific binding to $G A(C / T) A A G A(T / C) A A G A T A A$. Molecular and cellular biology 13: 4291-4300.

Dennis, G., Jr., B. T. Sherman, D. A. Hosack, J. Yang, W. Gao, H. C. Lane, and R. A. Lempicki. 2003. DAVID: Database for Annotation, Visualization, and Integrated Discovery. Genome biology 4: P3.

Dent, J. A., A. G. Polson, and M. W. Klymkowsky. 1989. A whole-mount immunocytochemical analysis of the expression of the intermediate filament protein vimentin in Xenopus. Development 105: 61-74.

Dettman, E. J., and M. J. Justice. 2008. The zinc finger SET domain gene Prdm14 is overexpressed in lymphoblastic lymphomas with retroviral insertions at Evi32. PloS one 3: e3823.

Dettman, E. J., S. J. Simko, B. Ayanga, B. L. Carofino, J. F. Margolin, H. C. Morse, 3rd, and M. J. Justice. 2011. Prdm14 initiates lymphoblastic leukemia after expanding a population of cells resembling common lymphoid progenitors. Oncogene 30: 2859-2873.

Ding, H. L., D. E. Clouthier, and K. B. Artinger. 2013. Redundant roles of PRDM family members in zebrafish craniofacial development. Developmental dynamics : an official publication of the American Association of Anatomists 242: 67-79.

Dobin, A., C. A. Davis, F. Schlesinger, J. Drenkow, C. Zaleski, S. Jha, P. Batut, M. Chaisson, and T. R. Gingeras. 2013. STAR: ultrafast universal RNA-seq aligner. Bioinformatics 29: 15-21.

Dottori, M., M. K. Gross, P. Labosky, and M. Goulding. 2001. The wingedhelix transcription factor Foxd3 suppresses interneuron differentiation and promotes neural crest cell fate. Development 128: 4127-4138.

Duan, Z., R. E. Person, H. H. Lee, S. Huang, J. Donadieu, R. Badolato, H. L. Grimes, T. Papayannopoulou, and M. S. Horwitz. 2007. Epigenetic regulation of protein-coding and microRNA genes by the Gfi1-interacting tumor suppressor PRDM5. Molecular and cellular biology 27: 6889-6902.

Dubois, L., L. Bally-Cuif, M. Crozatier, J. Moreau, L. Paquereau, and A. Vincent. 1998. XCoe2, a transcription factor of the Col/Olf-1/EBF family involved in the specification of primary neurons in Xenopus. Current biology : CB 8: 199-209.

Eagleson, G. W., and W. A. Harris. 1990. Mapping of the presumptive brain regions in the neural plate of Xenopus laevis. Journal of neurobiology 21: 427-440.

Eguchi, R., E. Yoshigai, T. Koga, S. Kuhara, and K. Tashiro. 2015. Spatiotemporal expression of Prdm genes during Xenopus development. Cytotechnology.

Ellis, P., B. M. Fagan, S. T. Magness, S. Hutton, O. Taranova, S. Hayashi, A. McMahon, M. Rao, and L. Pevny. 2004. SOX2, a persistent marker for multipotential neural stem cells derived from embryonic stem cells, the embryo or the adult. Developmental neuroscience 26: 148-165.

Endo, Y., N. Osumi, and Y. Wakamatsu. 2002. Bimodal functions of Notchmediated signaling are involved in neural crest formation during avian ectoderm development. Development 129: 863-873. 
Eom, G. H., K. Kim, S. M. Kim, H. J. Kee, J. Y. Kim, H. M. Jin, J. R. Kim, J. H. Kim, N. Choe, K. B. Kim, J. Lee, H. Kook, N. Kim, and S. B. Seo. 2009. Histone methyltransferase PRDM8 regulates mouse testis steroidogenesis. Biochemical and biophysical research communications 388: 131-136.

Fodde, R., and T. Brabletz. 2007. Wnt/beta-catenin signaling in cancer stemness and malignant behavior. Current opinion in cell biology 19: 150-158.

Fog, C. K., G. G. Galli, and A. H. Lund. 2012. PRDM proteins: important players in differentiation and disease. BioEssays : news and reviews in molecular, cellular and developmental biology 34: 50-60.

Friedmann, D. R., A. Aguilar, J. Fan, M. V. Nachury, and R. Marmorstein. 2012. Structure of the alpha-tubulin acetyltransferase, alphaTAT1, and implications for tubulin-specific acetylation. Proceedings of the National Academy of Sciences of the United States of America 109: 19655-19660.

Fujimi, T. J., M. Hatayama, and J. Aruga. 2012. Xenopus Zic3 controls notochord and organizer development through suppression of the Wnt/beta-catenin signaling pathway. Developmental biology 361: 220231.

Funabiki, T., B. L. Kreider, and J. N. Ihle. 1994. The carboxyl domain of zinc fingers of the Evi-1 myeloid transforming gene binds a consensus sequence of GAAGATGAG. Oncogene 9: 1575-1581.

Gammill, L. S., and M. Bronner-Fraser. 2002. Genomic analysis of neural crest induction. Development 129: 5731-5741.

Gammill, L. S., and M. Bronner-Fraser. 2003. Neural crest specification: migrating into genomics. Nature reviews. Neuroscience 4: 795-805.

Ge, W., F. He, K. J. Kim, B. Blanchi, V. Coskun, L. Nguyen, X. Wu, J. Zhao, J. I. Heng, K. Martinowich, J. Tao, H. Wu, D. Castro, M. M. Sobeih, G. Corfas, J. G. Gleeson, M. E. Greenberg, F. Guillemot, and Y. E. Sun. 2006. Coupling of cell migration with neurogenesis by proneural bHLH factors. Proceedings of the National Academy of Sciences of the United States of America 103: 1319-1324.

Geach, T. J., and L. B. Zimmerman. 2011. Developmental genetics in Xenopus tropicalis. Methods in molecular biology (Clifton, N.J.) 770: 77117.

Gleason, K. K., V. R. Dondeti, H. L. Hsia, E. R. Cochran, J. Gumulak-Smith, and M. S. Saha. 2003. The vesicular glutamate transporter 1 (xVGlut1) is expressed in discrete regions of the developing Xenopus laevis nervous system. Gene expression patterns : GEP 3: 503-507.

Goodfellow, M., N. E. Phillips, C. Manning, T. Galla, and N. Papalopulu. 2014. microRNA input into a neural ultradian oscillator controls emergence and timing of alternative cell states. Nature communications 5: 3399.

Grabole, N., J. Tischler, J. A. Hackett, S. Kim, F. Tang, H. G. Leitch, E. Magnusdottir, and M. A. Surani. 2013. Prdm14 promotes germline fate and naive pluripotency by repressing FGF signalling and DNA methylation. EMBO reports 14: 629-637.

Graham, V., J. Khudyakov, P. Ellis, and L. Pevny. 2003. SOX2 functions to maintain neural progenitor identity. Neuron 39: 749-765. 
Grainger, R. M. 2012. Xenopus tropicalis as a model organism for genetics and genomics: past, present, and future. Methods in molecular biology (Clifton, N.J.) 917: 3-15.

Groves, A. K., and C. LaBonne. 2014. Setting appropriate boundaries: fate, patterning and competence at the neural plate border. Developmental biology 389: 2-12.

Grunz, H., and L. Tacke. 1989. Neural differentiation of Xenopus laevis ectoderm takes place after disaggregation and delayed reaggregation without inducer. Cell differentiation and development : the official journal of the International Society of Developmental Biologists 28: 211-217.

Guo, X., T. Zhang, Z. Hu, Y. Zhang, Z. Shi, Q. Wang, Y. Cui, F. Wang, H. Zhao, and Y. Chen. 2014. Efficient RNA/Cas9-mediated genome editing in Xenopus tropicalis. Development 141: 707-714.

Hackett, J. A., S. Dietmann, K. Murakami, T. A. Down, H. G. Leitch, and M. A. Surani. 2013. Synergistic mechanisms of DNA demethylation during transition to ground-state pluripotency. Stem cell reports 1: 518-531.

Hanotel, J., N. Bessodes, A. Thelie, M. Hedderich, K. Parain, B. Van Driessche, O. Brandao Kde, S. Kricha, M. C. Jorgensen, A. GrapinBotton, P. Serup, C. Van Lint, M. Perron, T. Pieler, K. A. Henningfeld, and E. J. Bellefroid. 2014. The Prdm13 histone methyltransferase encoding gene is a Ptfla-Rbpj downstream target that suppresses glutamatergic and promotes GABAergic neuronal fate in the dorsal neural tube. Developmental biology 386: 340-357.

Hardcastle, Z., and N. Papalopulu. 2000. Distinct effects of XBF-1 in regulating the cell cycle inhibitor $\mathrm{p} 27(\mathrm{XIC1})$ and imparting a neural fate. Development 127: 1303-1314.

Harland, R. M. 1991. In situ hybridization: an improved whole-mount method for Xenopus embryos. Methods in cell biology 36: 685-695.

Hartenstein, V. 1989. Early neurogenesis in Xenopus: the spatio-temporal pattern of proliferation and cell lineages in the embryonic spinal cord. Neuron 3: 399-411.

Hartenstein, V. 1993. Early pattern of neuronal differentiation in the Xenopus embryonic brainstem and spinal cord. The Journal of comparative neurology 328: 213-231.

Hatakeyama, J., and R. Kageyama. 2006. Notch1 expression is spatiotemporally correlated with neurogenesis and negatively regulated by Notch1-independent Hes genes in the developing nervous system. Cereb Cortex 16 Suppl 1: i132-137.

Hayashi, K., K. Yoshida, and Y. Matsui. 2005. A histone H3 methyltransferase controls epigenetic events required for meiotic prophase. Nature 438: 374-378.

Heasman, J. 2002. Morpholino oligos: making sense of antisense? Developmental biology 243: 209-214.

Heasman, J., M. Kofron, and C. Wylie. 2000. Beta-catenin signaling activity dissected in the early Xenopus embryo: a novel antisense approach. Developmental biology 222: 124-134.

Hemmati-Brivanlou, A., and D. A. Melton. 1992. A truncated activin receptor inhibits mesoderm induction and formation of axial structures in Xenopus embryos. Nature 359: 609-614.

Hemmati-Brivanlou, A., and D. A. Melton. 1994. Inhibition of activin receptor signaling promotes neuralization in Xenopus. Cell 77: 273-281. 
Hemmati-Brivanlou, A., and D. Melton. 1997. Vertebrate neural induction. Annual review of neuroscience 20: 43-60.

Hemmati-Brivanlou, A., O. G. Kelly, and D. A. Melton. 1994. Follistatin, an antagonist of activin, is expressed in the Spemann organizer and displays direct neuralizing activity. Cell 77: 283-295.

Hernandez-Lagunas, L., D. R. Powell, J. Law, K. A. Grant, and K. B. Artinger. 2011. prdm1a and olig4 act downstream of Notch signaling to regulate cell fate at the neural plate border. Developmental biology 356: 496-505.

Hernandez-Lagunas, L., I. F. Choi, T. Kaji, P. Simpson, C. Hershey, Y. Zhou, L. Zon, M. Mercola, and K. B. Artinger. 2005. Zebrafish narrowminded disrupts the transcription factor prdm1 and is required for neural crest and sensory neuron specification. Developmental biology 278: 347-357.

Hirata, H., S. Yoshiura, T. Ohtsuka, Y. Bessho, T. Harada, K. Yoshikawa, and R. Kageyama. 2002. Oscillatory expression of the bHLH factor Hes1 regulated by a negative feedback loop. Science 298: 840-843.

Hohenauer, T., and A. W. Moore. 2012. The Prdm family: expanding roles in stem cells and development. Development 139: 2267-2282.

Holland, J. D., A. Klaus, A. N. Garratt, and W. Birchmeier. 2013. Wnt signaling in stem and cancer stem cells. Current opinion in cell biology 25: 254-264.

Hollemann, T., and T. Pieler. 1999. Xpitx-1: a homeobox gene expressed during pituitary and cement gland formation of Xenopus embryos. Mechanisms of development 88: 249-252.

Hong, C. S., and J. P. Saint-Jeannet. 2007. The activity of Pax3 and Zic1 regulates three distinct cell fates at the neural plate border. Molecular biology of the cell 18: 2192-2202.

Houtmeyers, R., J. Souopgui, S. Tejpar, and R. Arkell. 2013. The ZIC gene family encodes multi-functional proteins essential for patterning and morphogenesis. Cellular and molecular life sciences : CMLS 70: 37913811.

Huang, S. 2002. Histone methyltransferases, diet nutrients and tumour suppressors. Nature reviews. Cancer 2: 469-476.

Hubers, A. J., D. A. Heideman, S. A. Burgers, G. J. Herder, P. J. Sterk, R. J. Rhodius, H. J. Smit, F. Krouwels, A. Welling, B. I. Witte, S. Duin, R. Koning, E. F. Comans, R. D. Steenbergen, P. E. Postmus, G. A. Meijer, P. J. Snijders, E. F. Smit, and E. Thunnissen. 2015. DNA hypermethylation analysis in sputum for the diagnosis of lung cancer: training validation set approach. British journal of cancer 112: 1105-1113.

Imayoshi, I., and R. Kageyama. 2011. The role of Notch signaling in adult neurogenesis. Molecular neurobiology 44: 7-12.

Imayoshi, I., and R. Kageyama. 2014. bHLH factors in self-renewal, multipotency, and fate choice of neural progenitor cells. Neuron 82: 9-23.

Jessell, T. M. 2000. Neuronal specification in the spinal cord: inductive signals and transcriptional codes. Nature reviews. Genetics 1: 20-29.

Johnson, A. D., E. Richardson, R. F. Bachvarova, and B. I. Crother. 2011. Evolution of the germ line-soma relationship in vertebrate embryos. Reproduction (Cambridge, England) 141: 291-300. 
Kageyama, R., T. Ohtsuka, H. Shimojo, and I. Imayoshi. 2008. Dynamic Notch signaling in neural progenitor cells and a revised view of lateral inhibition. Nature neuroscience 11: 1247-1251.

Kengaku, M., and H. Okamoto. 1995. bFGF as a possible morphogen for the anteroposterior axis of the central nervous system in Xenopus. Development 121: 3121-3130.

Khudyakov, J., and M. Bronner-Fraser. 2009. Comprehensive spatiotemporal analysis of early chick neural crest network genes. Developmental dynamics : an official publication of the American Association of Anatomists 238: 716-723.

Kim, K. C., L. Geng, and S. Huang. 2003. Inactivation of a histone methyltransferase by mutations in human cancers. Cancer research 63: 7619-7623.

Kinameri, E., T. Inoue, J. Aruga, I. Imayoshi, R. Kageyama, T. Shimogori, and A. W. Moore. 2008. Prdm proto-oncogene transcription factor family expression and interaction with the Notch-Hes pathway in mouse neurogenesis. PloS one 3: e3859.

Kiyota, T., and T. Kinoshita. 2002. Cysteine-rich region of X-Serrate-1 is required for activation of Notch signaling in Xenopus primary neurogenesis. The International journal of developmental biology 46: 1057-1060.

Kiyota, T., H. Jono, S. Kuriyama, K. Hasegawa, S. Miyatani, and T. Kinoshita. 2001. X-Serrate-1 is involved in primary neurogenesis in Xenopus laevis in a complementary manner with X-Delta-1. Development genes and evolution 211: 367-376.

Klisch, T. 2006. Transcriptional control in the context of primary neurogenesis. Göttingen Center for Molecular Biosciences

Klymkowsky, M. W., C. C. Rossi, and K. B. Artinger. 2010. Mechanisms driving neural crest induction and migration in the zebrafish and Xenopus laevis. Cell adhesion \& migration 4: 595-608.

Kolm, P. J., and H. L. Sive. 1995. Efficient hormone-inducible protein function in Xenopus laevis. Developmental biology 171: 267-272.

Komai, T., H. Iwanari, Y. Mochizuki, T. Hamakubo, and Y. Shinkai. 2009. Expression of the mouse PR domain protein Prdm8 in the developing central nervous system. Gene expression patterns : GEP 9: 503-514.

Kondo, T., A. J. Matsuoka, A. Shimomura, K. R. Koehler, R. J. Chan, J. M. Miller, E. F. Srour, and E. Hashino. 2011. Wnt signaling promotes neuronal differentiation from mesenchymal stem cells through activation of Tlx3. Stem cells (Dayton, Ohio) 29: 836-846.

Korinek, V., N. Barker, P. J. Morin, D. van Wichen, R. de Weger, K. W. Kinzler, B. Vogelstein, and H. Clevers. 1997. Constitutive transcriptional activation by a beta-catenin-Tcf complex in APC-/- colon carcinoma. Science 275: 1784-1787.

Kouzarides, T. 2007. Chromatin modifications and their function. Cell 128: 693705.

Koyano-Nakagawa, N., and C. Kintner. 2005. The expression and function of MTG/ETO family proteins during neurogenesis. Developmental biology 278: 22-34.

Koyano-Nakagawa, N., J. Kim, D. Anderson, and C. Kintner. 2000. Hes6 acts in a positive feedback loop with the neurogenins to promote neuronal differentiation. Development 127: 4203-4216. 
Kroll, K. L., A. N. Salic, L. M. Evans, and M. W. Kirschner. 1998. Geminin, a neuralizing molecule that demarcates the future neural plate at the onset of gastrulation. Development 125: 3247-3258.

Kuo, T. C., and K. L. Calame. 2004. B lymphocyte-induced maturation protein (Blimp)-1, IFN regulatory factor (IRF)-1, and IRF-2 can bind to the same regulatory sites. J Immunol 173: 5556-5563.

Kuroda, H., O. Wessely, and E. M. De Robertis. 2004. Neural induction in Xenopus: requirement for ectodermal and endomesodermal signals via Chordin, Noggin, beta-Catenin, and Cerberus. PLoS biology 2: E92.

Kuroda, H., L. Fuentealba, A. Ikeda, B. Reversade, and E. M. De Robertis. 2005. Default neural induction: neuralization of dissociated Xenopus cells is mediated by Ras/MAPK activation. Genes \& development 19: 10221027.

Laemmli, U. K. 1970. Cleavage of structural proteins during the assembly of the head of bacteriophage T4. Nature 227: 680-685.

Lamb, T. M., and R. M. Harland. 1995. Fibroblast growth factor is a direct neural inducer, which combined with noggin generates anterior-posterior neural pattern. Development 121: 3627-3636.

Lamb, T. M., A. K. Knecht, W. C. Smith, S. E. Stachel, A. N. Economides, N. Stahl, G. D. Yancopolous, and R. M. Harland. 1993. Neural induction by the secreted polypeptide noggin. Science 262: 713-718.

Lamborghini, J. E. 1980. Rohon-beard cells and other large neurons in Xenopus embryos originate during gastrulation. The Journal of comparative neurology 189: 323-333.

Le Douarin, N. M., and M. A. Teillet. 1973. The migration of neural crest cells to the wall of the digestive tract in avian embryo. Journal of embryology and experimental morphology 30: 31-48.

Le Douarin, N. M., and J. Smith. 1988. Development of the peripheral nervous system from the neural crest. Annual review of cell biology 4: 375-404.

Le Douarin, N. M., S. Creuzet, G. Couly, and E. Dupin. 2004. Neural crest cell plasticity and its limits. Development 131: 4637-4650.

Le Dreau, G., and E. Marti. 2012. Dorsal-ventral patterning of the neural tube: a tale of three signals. Developmental neurobiology 72: 1471-1481.

Leclerc, C., I. Neant, and M. Moreau. 2012. The calcium: an early signal that initiates the formation of the nervous system during embryogenesis. Frontiers in molecular neuroscience 5: 3.

Lefebvre, V., B. Dumitriu, A. Penzo-Mendez, Y. Han, and B. Pallavi. 2007. Control of cell fate and differentiation by Sry-related high-mobility-group box (Sox) transcription factors. The international journal of biochemistry \& cell biology 39: 2195-2214.

Lei, Y., X. Guo, Y. Liu, Y. Cao, Y. Deng, X. Chen, C. H. Cheng, I. B. Dawid, Y. Chen, and H. Zhao. 2012. Efficient targeted gene disruption in Xenopus embryos using engineered transcription activator-like effector nucleases (TALENs). Proceedings of the National Academy of Sciences of the United States of America 109: 17484-17489.

Lewis, J. 1996. Neurogenic genes and vertebrate neurogenesis. Current opinion in neurobiology 6: 3-10.

Li, Y., and N. E. Baker. 2001. Proneural enhancement by Notch overcomes Suppressor-of-Hairless repressor function in the developing Drosophila eye. Current biology : CB 11: 330-338. 
Lindsell, C. E., J. Boulter, G. diSibio, A. Gossler, and G. Weinmaster. 1996. Expression patterns of Jagged, Delta1, Notch1, Notch2, and Notch3 genes identify ligand-receptor pairs that may function in neural development. Molecular and cellular neurosciences 8: 14-27.

Linker, C., and C. D. Stern. 2004. Neural induction requires BMP inhibition only as a late step, and involves signals other than FGF and Wnt antagonists. Development 131: 5671-5681.

Liu, C., W. Ma, W. Su, and J. Zhang. 2012. Prdm14 acts upstream of islet2 transcription to regulate axon growth of primary motoneurons in zebrafish. Development 139: 4591-4600.

Liu, Y., D. Luo, Y. Lei, W. Hu, H. Zhao, and C. H. Cheng. 2014. A highly effective TALEN-mediated approach for targeted gene disruption in Xenopus tropicalis and zebrafish. Methods 69: 58-66.

Louvi, A., and S. Artavanis-Tsakonas. 2006. Notch signalling in vertebrate neural development. Nature reviews. Neuroscience 7: 93-102.

Luo, T., Y. H. Lee, J. P. Saint-Jeannet, and T. D. Sargent. 2003. Induction of neural crest in Xenopus by transcription factor AP2alpha. Proceedings of the National Academy of Sciences of the United States of America 100: 532-537.

Ma, Q., C. Kintner, and D. J. Anderson. 1996. Identification of neurogenin, a vertebrate neuronal determination gene. Cell 87: 43-52.

Ma, Z., T. Swigut, A. Valouev, A. Rada-Iglesias, and J. Wysocka. 2011. Sequence-specific regulator Prdm14 safeguards mouse ESCs from entering extraembryonic endoderm fates. Nature structural \& molecular biology 18: 120-127.

McGrew, L. L., S. Hoppler, and R. T. Moon. 1997. Wnt and FGF pathways cooperatively pattern anteroposterior neural ectoderm in Xenopus. Mechanisms of development 69: 105-114.

Meani, N., F. Pezzimenti, G. Deflorian, M. Mione, and M. Alcalay. 2009. The tumor suppressor PRDM5 regulates Wnt signaling at early stages of zebrafish development. PloS one 4: e4273.

Merzdorf, C. S., and H. L. Sive. 2006. The zic1 gene is an activator of Wnt signaling. The International journal of developmental biology 50: 611617.

Meulemans, D., and M. Bronner-Fraser. 2004. Gene-regulatory interactions in neural crest evolution and development. Developmental cell 7: 291-299.

Milet, C., F. Maczkowiak, D. D. Roche, and A. H. Monsoro-Burq. 2013. Pax3 and Zic1 drive induction and differentiation of multipotent, migratory, and functional neural crest in Xenopus embryos. Proceedings of the National Academy of Sciences of the United States of America 110: 5528-5533.

Miyata, T., M. Okamoto, T. Shinoda, and A. Kawaguchi. 2014. Interkinetic nuclear migration generates and opposes ventricular-zone crowding: insight into tissue mechanics. Frontiers in cellular neuroscience 8: 473.

Mizuseki, K., M. Kishi, M. Matsui, S. Nakanishi, and Y. Sasai. 1998a. Xenopus Zic-related-1 and Sox-2, two factors induced by chordin, have distinct activities in the initiation of neural induction. Development 125: 579-587.

Mizuseki, K., M. Kishi, K. Shiota, S. Nakanishi, and Y. Sasai. 1998b. SoxD: an essential mediator of induction of anterior neural tissues in Xenopus embryos. Neuron 21: 77-85. 
Momiji, H., and N. A. Monk. 2009. Oscillatory Notch-pathway activity in a delay model of neuronal differentiation. Physical review. E, Statistical, nonlinear, and soft matter physics 80: 021930.

Monsoro-Burq, A. H., R. B. Fletcher, and R. M. Harland. 2003. Neural crest induction by paraxial mesoderm in Xenopus embryos requires FGF signals. Development 130: 3111-3124.

Monsoro-Burq, A. H., E. Wang, and R. Harland. 2005. Msx1 and Pax3 cooperate to mediate FGF8 and WNT signals during Xenopus neural crest induction. Developmental cell 8: 167-178.

Moody, S. A., and H. S. Je. 2002. Neural induction, neural fate stabilization, and neural stem cells. TheScientificWorldJournal 2: 1147-1166.

Moreau, M., I. Neant, S. E. Webb, A. L. Miller, and C. Leclerc. 2008. Calcium signalling during neural induction in Xenopus laevis embryos. Philosophical transactions of the Royal Society of London. Series B, Biological sciences 363: 1371-1375.

Moussa, H., and I. Sidhom. 2013. NKX2-5, SIL/TAL and TLX3/HOX11L2 expression in Egyptian pediatric T-cell acute lymphoblastic leukemia. Asia-Pacific journal of clinical oncology.

Munoz-Sanjuan, I., and A. H. Brivanlou. 2002. Neural induction, the default model and embryonic stem cells. Nature reviews. Neuroscience 3: 271280.

Nagy, V., T. Cole, C. Van Campenhout, T. M. Khoung, C. Leung, S. Vermeiren, M. Novatchkova, D. Wenzel, D. Cikes, A. A. Polyansky, I. Kozieradzki, A. Meixner, E. J. Bellefroid, G. G. Neely, and J. M. Penninger. 2015. The evolutionarily conserved transcription factor PRDM12 controls sensory neuron development and pain perception. Cell Cycle: 0 .

Nakaki, F., and M. Saitou. 2014. PRDM14: a unique regulator for pluripotency and epigenetic reprogramming. Trends in biochemical sciences 39: 289298.

Nakata, K., T. Nagai, J. Aruga, and K. Mikoshiba. 1997. Xenopus Zic3, a primary regulator both in neural and neural crest development. Proceedings of the National Academy of Sciences of the United States of America 94: 11980-11985.

Nakata, K., T. Nagai, J. Aruga, and K. Mikoshiba. 1998. Xenopus Zic family and its role in neural and neural crest development. Mechanisms of development 75: 43-51.

Nakayama, T., M. B. Fish, M. Fisher, J. Oomen-Hajagos, G. H. Thomsen, and R. M. Grainger. 2013. Simple and efficient CRISPR/Cas9-mediated targeted mutagenesis in Xenopus tropicalis. Genesis 51: 835-843.

Nelson, B. R., and T. A. Reh. 2008. Relationship between Delta-like and proneural bHLH genes during chick retinal development. Developmental dynamics : an official publication of the American Association of Anatomists 237: 1565-1580.

Nguyen, L., A. Besson, J. I. Heng, C. Schuurmans, L. Teboul, C. Parras, A. Philpott, J. M. Roberts, and F. Guillemot. 2006. p27kip1 independently promotes neuronal differentiation and migration in the cerebral cortex. Genes \& development 20: 1511-1524.

Nichane, M., N. de Croze, X. Ren, J. Souopgui, A. H. Monsoro-Burq, and E. J. Bellefroid. 2008. Hairy2-Id3 interactions play an essential role in 
Xenopus neural crest progenitor specification. Developmental biology 322: 355-367.

Nieber, F., T. Pieler, and K. A. Henningfeld. 2009. Comparative expression analysis of the neurogenins in Xenopus tropicalis and Xenopus laevis. Developmental dynamics : an official publication of the American Association of Anatomists 238: 451-458.

Nieuwkoop, P.D., Faber, J. (Eds.) (1967). Normal table of Xenopus laevis (Daudin). Second Edition. North Holland Publ. Co. Amsterdam.

Nikitina, N., T. Sauka-Spengler, and M. Bronner-Fraser. 2008. Dissecting early regulatory relationships in the lamprey neural crest gene network. Proceedings of the National Academy of Sciences of the United States of America 105: 20083-20088.

Nishikawa, N., M. Toyota, H. Suzuki, T. Honma, T. Fujikane, T. Ohmura, T. Nishidate, M. Ohe-Toyota, R. Maruyama, T. Sonoda, Y. Sasaki, T. Urano, K. Imai, K. Hirata, and T. Tokino. 2007. Gene amplification and overexpression of PRDM14 in breast cancers. Cancer research 67: 9649-9657.

Nutt, S. L., O. J. Bronchain, K. O. Hartley, and E. Amaya. 2001. Comparison of morpholino based translational inhibition during the development of Xenopus laevis and Xenopus tropicalis. Genesis 30: 110-113.

Ohinata, Y., H. Ohta, M. Shigeta, K. Yamanaka, T. Wakayama, and M. Saitou. 2009. A signaling principle for the specification of the germ cell lineage in mice. Cell 137: 571-584.

Ohinata, Y., B. Payer, D. O'Carroll, K. Ancelin, Y. Ono, M. Sano, S. C. Barton, T. Obukhanych, M. Nussenzweig, A. Tarakhovsky, M. Saitou, and M. A. Surani. 2005. Blimp1 is a critical determinant of the germ cell lineage in mice. Nature 436: 207-213.

Ohkawara, B., and C. Niehrs. 2011. An ATF2-based luciferase reporter to monitor non-canonical Wnt signaling in Xenopus embryos. Developmental dynamics : an official publication of the American Association of Anatomists 240: 188-194.

Okashita, N., Y. Kumaki, K. Ebi, M. Nishi, Y. Okamoto, M. Nakayama, S. Hashimoto, T. Nakamura, K. Sugasawa, N. Kojima, T. Takada, M. Okano, and Y. Seki. 2014. PRDM14 promotes active DNA demethylation through the ten-eleven translocation (TET)-mediated base excision repair pathway in embryonic stem cells. Development 141: 269280.

Olesnicky, E., L. Hernandez-Lagunas, and K. B. Artinger. 2010. prdm1a Regulates sox10 and islet1 in the development of neural crest and Rohon-Beard sensory neurons. Genesis 48: 656-666.

Olson, E. C., A. F. Schinder, J. L. Dantzker, E. A. Marcus, N. C. Spitzer, and W. A. Harris. 1998. Properties of ectopic neurons induced by Xenopus neurogenin1 misexpression. Molecular and cellular neurosciences 12: 281-299.

Ozair, M. Z., C. Kintner, and A. H. Brivanlou. 2013. Neural induction and early patterning in vertebrates. Wiley interdisciplinary reviews. Developmental biology 2: 479-498.

Papalopulu, N., and C. Kintner. 1996. A posteriorising factor, retinoic acid, reveals that anteroposterior patterning controls the timing of neuronal differentiation in Xenopus neuroectoderm. Development 122: 3409-3418. 
Papanayotou, C., A. Mey, A. M. Birot, Y. Saka, S. Boast, J. C. Smith, J. Samarut, and C. D. Stern. 2008. A mechanism regulating the onset of Sox2 expression in the embryonic neural plate. PLoS biology 6: e2.

Park, D. S., J. H. Seo, M. Hong, W. Bang, J. K. Han, and S. C. Choi. 2013. Role of Sp5 as an essential early regulator of neural crest specification in xenopus. Developmental dynamics : an official publication of the American Association of Anatomists 242: 1382-1394.

Patterson, K. D., and P. A. Krieg. 1999. Hox11-family genes XHox11 and XHox11L2 in xenopus: XHox11L2 expression is restricted to a subset of the primary sensory neurons. Developmental dynamics : an official publication of the American Association of Anatomists 214: 34-43.

Pegoraro, C., and A. H. Monsoro-Burq. 2013. Signaling and transcriptional regulation in neural crest specification and migration: lessons from xenopus embryos. Wiley interdisciplinary reviews. Developmental biology 2: 247-259.

Penzel, R., R. Oschwald, Y. Chen, L. Tacke, and H. Grunz. 1997. Characterization and early embryonic expression of a neural specific transcription factor XSOX3 in Xenopus laevis. The International journal of developmental biology 41: 667-677.

Pera, E. M., A. Ikeda, E. Eivers, and E. M. De Robertis. 2003. Integration of IGF, FGF, and anti-BMP signals via Smad1 phosphorylation in neural induction. Genes \& development 17: 3023-3028.

Pera, E. M., H. Acosta, N. Gouignard, M. Climent, and I. Arregi. 2014. Active signals, gradient formation and regional specificity in neural induction. Experimental cell research 321: 25-31.

Perez, S. E., S. Rebelo, and D. J. Anderson. 1999. Early specification of sensory neuron fate revealed by expression and function of neurogenins in the chick embryo. Development 126: 1715-1728.

Perron, M., K. Opdecamp, K. Butler, W. A. Harris, and E. J. Bellefroid. 1999. X-ngnr-1 and Xath3 promote ectopic expression of sensory neuron markers in the neurula ectoderm and have distinct inducing properties in the retina. Proceedings of the National Academy of Sciences of the United States of America 96: 14996-15001.

Pevny, L., and M. Placzek. 2005. SOX genes and neural progenitor identity. Current opinion in neurobiology 15: 7-13.

Piccolo, S., Y. Sasai, B. Lu, and E. M. De Robertis. 1996. Dorsoventral patterning in Xenopus: inhibition of ventral signals by direct binding of chordin to BMP-4. Cell 86: 589-598.

Piccolo, S., E. Agius, L. Leyns, S. Bhattacharyya, H. Grunz, T. Bouwmeester, and E. M. De Robertis. 1999. The head inducer Cerberus is a multifunctional antagonist of Nodal, BMP and Wnt signals. Nature 397: 707-710.

Pinheiro, I., R. Margueron, N. Shukeir, M. Eisold, C. Fritzsch, F. M. Richter, G. Mittler, C. Genoud, S. Goyama, M. Kurokawa, J. Son, D. Reinberg, M. Lachner, and T. Jenuwein. 2012. Prdm3 and Prdm16 are H3K9me1 methyltransferases required for mammalian heterochromatin integrity. Cell 150: 948-960.

Plouhinec, J. L., D. D. Roche, C. Pegoraro, A. L. Figueiredo, F. Maczkowiak, L. J. Brunet, C. Milet, J. P. Vert, N. Pollet, R. M. Harland, and A. H. Monsoro-Burq. 2014. Pax3 and Zic1 trigger the 
early neural crest gene regulatory network by the direct activation of multiple key neural crest specifiers. Developmental biology 386: 461-472.

Pohl, B. S., and W. Knochel. 2001. Overexpression of the transcriptional repressor FoxD3 prevents neural crest formation in Xenopus embryos. Mechanisms of development 103: 93-106.

Pourebrahim, R., R. Houtmeyers, S. Ghogomu, S. Janssens, A. Thelie, H. T. Tran, T. Langenberg, K. Vleminckx, E. Bellefroid, J. J. Cassiman, and S. Tejpar. 2011. Transcription factor Zic2 inhibits Wnt/beta-catenin protein signaling. The Journal of biological chemistry 286: 37732-37740.

Powell, D. R., L. Hernandez-Lagunas, K. LaMonica, and K. B. Artinger. 2013. Prdm1a directly activates foxd3 and tfap2a during zebrafish neural crest specification. Development 140: 3445-3455.

Powell, L. M., and A. P. Jarman. 2008. Context dependence of proneural bHLH proteins. Current opinion in genetics \& development 18: 411-417.

Pozzoli, O., A. Bosetti, L. Croci, G. G. Consalez, and M. L. Vetter. 2001. Xebf3 is a regulator of neuronal differentiation during primary neurogenesis in Xenopus. Developmental biology 233: 495-512.

Rea, S., F. Eisenhaber, D. O'Carroll, B. D. Strahl, Z. W. Sun, M. Schmid, S. Opravil, K. Mechtler, C. P. Ponting, C. D. Allis, and T. Jenuwein. 2000. Regulation of chromatin structure by site-specific histone $\mathrm{H} 3$ methyltransferases. Nature 406: 593-599.

Roberts, A. 2000. Early functional organization of spinal neurons in developing lower vertebrates. Brain research bulletin 53: 585-593.

Roberts, A., W. C. Li, and S. R. Soffe. 2012. A functional scaffold of CNS neurons for the vertebrates: the developing Xenopus laevis spinal cord. Developmental neurobiology 72: 575-584.

Rogers, C. D., S. A. Moody, and E. S. Casey. 2009. Neural induction and factors that stabilize a neural fate. Birth defects research. Part C, Embryo today : reviews 87: 249-262.

Ross, S. E., A. E. McCord, C. Jung, D. Atan, S. I. Mok, M. Hemberg, T. K. Kim, J. Salogiannis, L. Hu, S. Cohen, Y. Lin, D. Harrar, R. R. Mclnnes, and M. E. Greenberg. 2012. Bhlhb5 and Prdm8 form a repressor complex involved in neuronal circuit assembly. Neuron 73: 292-303.

Rossi, C. C., T. Kaji, and K. B. Artinger. 2009. Transcriptional control of Rohon-Beard sensory neuron development at the neural plate border. Developmental dynamics : an official publication of the American Association of Anatomists 238: 931-943.

Rossi, C. C., L. Hernandez-Lagunas, C. Zhang, I. F. Choi, L. Kwok, M. Klymkowsky, and K. B. Artinger. 2008. Rohon-Beard sensory neurons are induced by BMP4 expressing non-neural ectoderm in Xenopus laevis. Developmental biology 314: 351-361.

Ruark, E., S. Seal, H. McDonald, F. Zhang, A. Elliot, K. Lau, E. Perdeaux, E. Rapley, R. Eeles, J. Peto, Z. Kote-Jarai, K. Muir, J. Nsengimana, J. Shipley, D. T. Bishop, M. R. Stratton, D. F. Easton, R. A. Huddart, N. Rahman, and C. Turnbull. 2013. Identification of nine new susceptibility loci for testicular cancer, including variants near DAZL and PRDM14. Nature genetics 45: 686-689.

Rubenstein, A., J. Merriam, and M. W. Klymkowsky. 1997. Localizing the adhesive and signaling functions of plakoglobin. Developmental genetics 20: 91-102. 
Saha, M. S., R. R. Miles, and R. M. Grainger. 1997. Dorsal-ventral patterning during neural induction in Xenopus: assessment of spinal cord regionalization with XHB9, a marker for the motor neuron region. Developmental biology 187: 209-223.

Sambrook, J. and Russel, D.W. (Eds.) (2001). Molecular Cloning: a laboratory manual. Third Edition. Cold Spring Harbour Laboratory Press, Cold Spring Harbour, New York.

Sanchez-Ferras, O., B. Coutaud, T. Djavanbakht Samani, I. Tremblay, 0. Souchkova, and N. Pilon. 2012. Caudal-related homeobox (Cdx) protein-dependent integration of canonical Wnt signaling on paired-box 3 (Pax3) neural crest enhancer. The Journal of biological chemistry 287: 16623-16635.

Sanger, F., S. Nicklen, and A. R. Coulson. 1977. DNA sequencing with chainterminating inhibitors. Proceedings of the National Academy of Sciences of the United States of America 74: 5463-5467.

Sasai, Y. 1998. Identifying the missing links: genes that connect neural induction and primary neurogenesis in vertebrate embryos. Neuron 21: 455-458.

Sasai, Y., B. Lu, H. Steinbeisser, D. Geissert, L. K. Gont, and E. M. De Robertis. 1994. Xenopus chordin: a novel dorsalizing factor activated by organizer-specific homeobox genes. Cell 79: 779-790.

Sato, S. M., and T. D. Sargent. 1989. Development of neural inducing capacity in dissociated Xenopus embryos. Developmental biology 134: 263-266.

Sato, T., N. Sasai, and Y. Sasai. 2005. Neural crest determination by coactivation of Pax3 and Zic1 genes in Xenopus ectoderm. Development 132: 2355-2363.

Sayitoglu, M., Y. Erbilgin, O. Hatirnaz Ng, I. Yildiz, T. Celkan, S. Anak, O. Devecioglu, G. Aydogan, S. Karaman, N. Sarper, C. Timur, U. Ure, and U. Ozbek. 2012. Upregulation of T-Cell-Specific Transcription Factor Expression in Pediatric T-Cell Acute Lymphoblastic Leukemia (T-ALL). Turkish journal of haematology : official journal of Turkish Society of Haematology 29: 325-333.

Schneider, M. L., D. L. Turner, and M. L. Vetter. 2001. Notch signaling can inhibit Xath5 function in the neural plate and developing retina. Molecular and cellular neurosciences 18: 458-472.

Schroeter, E. H., J. A. Kisslinger, and R. Kopan. 1998. Notch-1 signalling requires ligand-induced proteolytic release of intracellular domain. Nature 393: 382-386.

Seale, P., S. Kajimura, W. Yang, S. Chin, L. M. Rohas, M. Uldry, G. Tavernier, D. Langin, and B. M. Spiegelman. 2007. Transcriptional control of brown fat determination by PRDM16. Cell metabolism 6: 38-54.

Selkoe, D., and R. Kopan. 2003. Notch and Presenilin: regulated intramembrane proteolysis links development and degeneration. Annual review of neuroscience 26: 565-597.

Seo, S., and K. L. Kroll. 2006. Geminin's double life: chromatin connections that regulate transcription at the transition from proliferation to differentiation. Cell Cycle 5: 374-379.

Sharp, P. A., B. Sugden, and J. Sambrook. 1973. Detection of two restriction endonuclease activities in Haemophilus parainfluenzae using analytical agarose--ethidium bromide electrophoresis. Biochemistry 12: 3055-3063. 
Shimojo, H., T. Ohtsuka, and R. Kageyama. 2008. Oscillations in notch signaling regulate maintenance of neural progenitors. Neuron 58: 52-64.

Shu, X. S., H. Geng, L. Li, J. Ying, C. Ma, Y. Wang, F. F. Poon, X. Wang, Y. Ying, W. Yeo, G. Srivastava, S. W. Tsao, J. Yu, J. J. Sung, S. Huang, A. T. Chan, and Q. Tao. 2011. The epigenetic modifier PRDM5 functions as a tumor suppressor through modulating WNT/beta-catenin signaling and is frequently silenced in multiple tumors. Plos one 6: e27346.

Simko, S. J., H. Voicu, B. L. Carofino, and M. J. Justice. 2012. Mouse Lymphoblastic Leukemias Induced by Aberrant Expression Demonstrate Widespread Copy Number Alterations Also Found in Human ALL. Cancers 4: 1050-1066.

Simoes-Costa, M., and M. E. Bronner. 2015. Establishing neural crest identity: a gene regulatory recipe. Development 142: 242-257.

Sive, H. L., R. M. Grainger, and R. M. Harland. 2010. Microinjection of Xenopus embryos. Cold Spring Harbor protocols 2010: pdb ip81.

Smith, W. C., and R. M. Harland. 1992. Expression cloning of noggin, a new dorsalizing factor localized to the Spemann organizer in Xenopus embryos. Cell 70: 829-840.

Snellenberg, S., S. A. Cillessen, W. Van Criekinge, L. Bosch, C. J. Meijer, P. J. Snijders, and R. D. Steenbergen. 2014. Methylation-mediated repression of PRDM14 contributes to apoptosis evasion in HPV-positive cancers. Carcinogenesis 35: 2611-2618.

Solter, M., M. Koster, T. Hollemann, A. Brey, T. Pieler, and W. Knochel. 1999. Characterization of a subfamily of related winged helix genes, XFD-12/12'/12" (XFLIP), during Xenopus embryogenesis. Mechanisms of development 89: 161-165.

Souopgui, J., M. Solter, and T. Pieler. 2002. XPak3 promotes cell cycle withdrawal during primary neurogenesis in Xenopus laevis. The EMBO journal 21: 6429-6439.

Spear, P. C., and C. A. Erickson. 2012. Interkinetic nuclear migration: a mysterious process in search of a function. Development, growth \& differentiation 54: 306-316.

Stern, C. D. 2005. Neural induction: old problem, new findings, yet more questions. Development 132: 2007-2021.

Streit, A., A. J. Berliner, C. Papanayotou, A. Sirulnik, and C. D. Stern. 2000. Initiation of neural induction by FGF signalling before gastrulation. Nature 406: 74-78.

Sullivan, S. A., L. Akers, and S. A. Moody. 2001. foxD5a, a Xenopus winged helix gene, maintains an immature neural ectoderm via transcriptional repression that is dependent on the C-terminal domain. Developmental biology 232: 439-457.

Sun, X. J., P. F. Xu, T. Zhou, M. Hu, C. T. Fu, Y. Zhang, Y. Jin, Y. Chen, S. J. Chen, Q. H. Huang, T. X. Liu, and Z. Chen. 2008. Genome-wide survey and developmental expression mapping of zebrafish SET domaincontaining genes. PloS one 3: e1499.

Taverna, E., and W. B. Huttner. 2010. Neural progenitor nuclei IN motion. Neuron 67: 906-914.

Towbin, H., T. Staehelin, and J. Gordon. 1992. Electrophoretic transfer of proteins from polyacrylamide gels to nitrocellulose sheets: procedure and some applications. 1979. Biotechnology (Reading, Mass.) 24: 145-149. 
Tsuneyoshi, N., T. Sumi, H. Onda, H. Nojima, N. Nakatsuji, and H. Suemori. 2008. PRDM14 suppresses expression of differentiation marker genes in human embryonic stem cells. Biochemical and biophysical research communications 367: 899-905.

Uchikawa, M., Y. Kamachi, and H. Kondoh. 1999. Two distinct subgroups of Group B Sox genes for transcriptional activators and repressors: their expression during embryonic organogenesis of the chicken. Mechanisms of development 84: 103-120.

van der Sanden, M. H., H. Meems, M. Houweling, J. B. Helms, and A. B. Vaandrager. 2004. Induction of CCAAT/enhancer-binding protein (C/EBP)-homologous protein/growth arrest and DNA damage-inducible protein 153 expression during inhibition of phosphatidylcholine synthesis is mediated via activation of a C/EBP-activating transcription factorresponsive element. The Journal of biological chemistry 279: 5200752015.

Vasconcelos, F. F., and D. S. Castro. 2014. Transcriptional control of vertebrate neurogenesis by the proneural factor Ascl1. Frontiers in cellular neuroscience 8: 412.

Vernon, A. E., and A. Philpott. 2003. A single cdk inhibitor, p27Xic1, functions beyond cell cycle regulation to promote muscle differentiation in Xenopus. Development 130: 71-83.

Wang, S., and B. A. Barres. 2000. Up a notch: instructing gliogenesis. Neuron 27: 197-200.

Wettstein, D. A., D. L. Turner, and C. Kintner. 1997. The Xenopus homolog of Drosophila Suppressor of Hairless mediates Notch signaling during primary neurogenesis. Development 124: 693-702.

Wilson, P. A., and A. Hemmati-Brivanlou. 1995. Induction of epidermis and inhibition of neural fate by Bmp-4. Nature 376: 331-333.

Wu, Y., J. E. Ferguson, 3rd, H. Wang, R. Kelley, R. Ren, H. McDonough, J. Meeker, P. C. Charles, and C. Patterson. 2008. PRDM6 is enriched in vascular precursors during development and inhibits endothelial cell proliferation, survival, and differentiation. Journal of molecular and cellular cardiology 44: 47-58.

Wullimann, M. F., E. Rink, P. Vernier, and G. Schlosser. 2005. Secondary neurogenesis in the brain of the African clawed frog, Xenopus laevis, as revealed by PCNA, Delta-1, Neurogenin-related-1, and NeuroD expression. The Journal of comparative neurology 489: 387-402.

Yabuta, Y., K. Kurimoto, Y. Ohinata, Y. Seki, and M. Saitou. 2006. Gene expression dynamics during germline specification in mice identified by quantitative single-cell gene expression profiling. Biology of reproduction 75: 705-716.

Yamaji, M., Y. Seki, K. Kurimoto, Y. Yabuta, M. Yuasa, M. Shigeta, K. Yamanaka, Y. Ohinata, and M. Saitou. 2008. Critical function of Prdm14 for the establishment of the germ cell lineage in mice. Nature genetics 40: 1016-1022.

Yamaji, M., J. Ueda, K. Hayashi, H. Ohta, Y. Yabuta, K. Kurimoto, R. Nakato, Y. Yamada, K. Shirahige, and M. Saitou. 2013. PRDM14 ensures naive pluripotency through dual regulation of signaling and epigenetic pathways in mouse embryonic stem cells. Cell stem cell 12: 368-382. 
Yardley, N., and M. I. Garcia-Castro. 2012. FGF signaling transforms nonneural ectoderm into neural crest. Developmental biology 372: 166-177.

Zannino, D. A., G. B. Downes, and C. G. Sagerstrom. 2014. prdm $12 b$ specifies the $\mathrm{p} 1$ progenitor domain and reveals a role for V1 interneurons in swim movements. Developmental biology 390: 247-260.

Zhang, T., L. Meng, W. Dong, H. Shen, S. Zhang, Q. Liu, and J. Du. 2013. High expression of PRDM14 correlates with cell differentiation and is a novel prognostic marker in resected non-small cell lung cancer. Medical oncology (Northwood, London, England) 30: 605.

Zwane, T. B., and N. V. Nikitina. 2015. Spatiotemporal expression analysis of Prdm1 and Prdm1 binding partners in early chick embryo. Gene expression patterns : GEP 17: 56-68. 


\section{Appendix}

\subsection{Candidate gene list for the RNA-sequencing analysis of prdm14-GR overexpressing animal caps}

Given are the genes which were differentially expressed between prdm14-GR overexpressing animal caps and uninjected control caps, equivalent to stage 14 and 27 , in three independent experiments.

\subsubsection{Differentially expressed genes at stage 14}

Given are the ensembl id, official gene name, log2 fold change and the adjusted P-value with respect to multiple testing using the FDR method. Genes were sorted according to the highest and lowest log2 fold change, respectively.

Table 6.1 Summary of differentially expressed genes at stage 14

\begin{tabular}{|c|c|c|c|}
\hline \multicolumn{4}{|c|}{ upregulated } \\
\hline ensembl id & Gene & $\begin{array}{l}\text { log2 } \\
\text { fold } \\
\text { change } \\
\end{array}$ & $\begin{array}{l}\text { adjust. } \\
\text { P-value } \\
\end{array}$ \\
\hline $\begin{array}{l}\text { ENSXETG00000 } \\
015915\end{array}$ & prdm14 & 9.33 & 1.55E-68 \\
\hline $\begin{array}{l}\text { ENSXETG00000 } \\
010282\end{array}$ & $c d \times 1$ & 8.98 & 3.26E-29 \\
\hline $\begin{array}{l}\text { ENSXETG00000 } \\
024458\end{array}$ & zic3 & 8.23 & $3.45 E-23$ \\
\hline $\begin{array}{l}\text { ENSXETG00000 } \\
004589\end{array}$ & $c d x 4$ & 8.14 & $1.08 E-46$ \\
\hline $\begin{array}{l}\text { ENSXETG00000 } \\
025525\end{array}$ & pnhd & 7.71 & 2.67E-18 \\
\hline $\begin{array}{l}\text { ENSXETG00000 } \\
025407\end{array}$ & sp5 & 7.21 & 3.15E-39 \\
\hline $\begin{array}{l}\text { ENSXETG00000 } \\
003826\end{array}$ & wnt8a & 7.13 & $1.02 \mathrm{E}-18$ \\
\hline $\begin{array}{l}\text { ENSXETG00000 } \\
001801\end{array}$ & hoxd1 & 6.60 & 4.96E-17 \\
\hline $\begin{array}{l}\text { ENSXETG00000 } \\
007898\end{array}$ & neurog1 & 6.52 & 1.17E-10 \\
\hline $\begin{array}{l}\text { ENSXETG00000 } \\
019220\end{array}$ & bmpr1b & 5.80 & 1.02E-09 \\
\hline $\begin{array}{l}\text { ENSXETG00000 } \\
022950\end{array}$ & zbtb16 & 5.79 & 4.80E-18 \\
\hline $\begin{array}{l}\text { ENSXETG00000 } \\
022080\end{array}$ & $e m \times 2$ & 5.66 & $1.72 \mathrm{E}-29$ \\
\hline $\begin{array}{l}\text { ENSXETG00000 } \\
011788\end{array}$ & $c d x 2$ & 5.57 & 1.96E-22 \\
\hline $\begin{array}{l}\text { ENSXETG00000 } \\
007640\end{array}$ & pax3 & 5.51 & 8.74E-08 \\
\hline $\begin{array}{l}\text { ENSXETG00000 } \\
031623\end{array}$ & wnt10a & 5.39 & 2.05E-12 \\
\hline $\begin{array}{l}\text { ENSXETG00000 } \\
023986\end{array}$ & barhl2 & 5.29 & 4.73E-07 \\
\hline $\begin{array}{l}\text { ENSXETG00000 } \\
000742\end{array}$ & $\operatorname{Im} \times 1 b .1$ & 5.18 & 2.26E-13 \\
\hline $\begin{array}{l}\text { ENSXETG00000 } \\
002761\end{array}$ & fgf3 & 5.17 & 1.30E-06 \\
\hline $\begin{array}{l}\text { ENSXETG00000 } \\
020167\end{array}$ & tox & 4.94 & 7.99E-29 \\
\hline
\end{tabular}

\begin{tabular}{|c|c|c|c|}
\hline \multicolumn{4}{|c|}{ downregulated } \\
\hline ensembl id & Gene & $\begin{array}{l}\text { log2 } \\
\text { fold } \\
\text { change }\end{array}$ & $\begin{array}{l}\text { adjust. } \\
\text { P-value }\end{array}$ \\
\hline $\begin{array}{l}\text { ENSXETG00000 } \\
014812\end{array}$ & htr1a & -3.44 & $5.72 \mathrm{E}-06$ \\
\hline $\begin{array}{l}\text { ENSXETG00000 } \\
001728\end{array}$ & slc6a3 & -3.08 & 5.28E-04 \\
\hline $\begin{array}{l}\text { ENSXETG00000 } \\
027551\end{array}$ & ces5a & -3.02 & 4.91E-03 \\
\hline $\begin{array}{l}\text { ENSXETG00000 } \\
009166\end{array}$ & hmp19 & -2.77 & 1.97E-05 \\
\hline $\begin{array}{l}\text { ENSXETG00000 } \\
017117\end{array}$ & vstm5 & -2.75 & $3.44 \mathrm{E}-03$ \\
\hline $\begin{array}{l}\text { ENSXETG00000 } \\
012215\end{array}$ & hhex & -2.67 & 2.49E-03 \\
\hline $\begin{array}{l}\text { ENSXETG00000 } \\
022118\end{array}$ & gpr37 & -2.63 & 1.30E-14 \\
\hline $\begin{array}{l}\text { ENSXETG00000 } \\
011262\end{array}$ & c20orf85 & -2.55 & $2.42 \mathrm{E}-03$ \\
\hline $\begin{array}{l}\text { ENSXETG00000 } \\
032792\end{array}$ & gnrh2 & -2.50 & 4.63E-05 \\
\hline $\begin{array}{l}\text { ENSXETG00000 } \\
023176\end{array}$ & prox1 & -2.50 & 1.11E-03 \\
\hline $\begin{array}{l}\text { ENSXETG00000 } \\
014758\end{array}$ & dner & -2.47 & 4.76E-06 \\
\hline $\begin{array}{l}\text { ENSXETG00000 } \\
017493\end{array}$ & tmtops & -2.46 & 1.79E-03 \\
\hline $\begin{array}{l}\text { ENSXETG00000 } \\
004406\end{array}$ & & -2.44 & 2.87E-02 \\
\hline $\begin{array}{l}\text { ENSXETG00000 } \\
005224\end{array}$ & & -2.36 & 5.23E-04 \\
\hline $\begin{array}{l}\text { ENSXETG00000 } \\
033983\end{array}$ & dmrt2 & -2.31 & 3.83E-11 \\
\hline $\begin{array}{l}\text { ENSXETG00000 } \\
013115\end{array}$ & kiaa0895 & -2.31 & 5.83E-04 \\
\hline $\begin{array}{l}\text { ENSXETG00000 } \\
033308\end{array}$ & efhb & -2.31 & 5.97E-09 \\
\hline $\begin{array}{l}\text { ENSXETG00000 } \\
013407\end{array}$ & aatk & -2.30 & 2.02E-04 \\
\hline $\begin{array}{l}\text { ENSXETG00000 } \\
001353\end{array}$ & hmcn2 & -2.30 & 1.80E-02 \\
\hline
\end{tabular}




\begin{tabular}{|c|c|c|c|}
\hline $\begin{array}{l}\text { ENSXETG00000 } \\
025183\end{array}$ & hoxc5 & 4.77 & 1.25E-27 \\
\hline $\begin{array}{l}\text { ENSXETG00000 } \\
023476\end{array}$ & hoxc8 & 4.74 & 1.69E-29 \\
\hline $\begin{array}{l}\text { ENSXETG00000 } \\
000722\end{array}$ & hoxa7 & 4.70 & 9.15E-11 \\
\hline $\begin{array}{l}\text { ENSXETG00000 } \\
009083\end{array}$ & hes7.2 & 4.69 & 5.01E-06 \\
\hline $\begin{array}{l}\text { ENSXETG00000 } \\
000802\end{array}$ & ror2 & 4.55 & 3.83E-31 \\
\hline $\begin{array}{l}\text { ENSXETG00000 } \\
000724\end{array}$ & hoxa9 & 4.54 & 4.99E-07 \\
\hline $\begin{array}{l}\text { ENSXETG00000 } \\
019927\end{array}$ & crabp2 & 4.52 & $3.42 \mathrm{E}-05$ \\
\hline $\begin{array}{l}\text { ENSXETG00000 } \\
000237\end{array}$ & $z e b 2$ & 4.48 & 4.04E-06 \\
\hline $\begin{array}{l}\text { ENSXETG00000 } \\
023479\end{array}$ & hoxc6 & 4.41 & 3.07E-19 \\
\hline $\begin{array}{l}\text { ENSXETG00000 } \\
023475\end{array}$ & hoxc9 & 4.38 & $1.83 \mathrm{E}-16$ \\
\hline $\begin{array}{l}\text { ENSXETG00000 } \\
018660\end{array}$ & $t p b g$ & 4.31 & $2.80 \mathrm{E}-24$ \\
\hline $\begin{array}{l}\text { ENSXETG00000 } \\
021703\end{array}$ & $\mathrm{fmn} / 2$ & 4.30 & 1.41E-06 \\
\hline $\begin{array}{l}\text { ENSXETG00000 } \\
001068\end{array}$ & gcm2 & 4.29 & 3.43E-05 \\
\hline $\begin{array}{l}\text { ENSXETG00000 } \\
000728\end{array}$ & hoxa10 & 4.28 & $3.14 \mathrm{E}-05$ \\
\hline $\begin{array}{l}\text { ENSXETG00000 } \\
010153\end{array}$ & $e b f 2$ & 4.27 & $1.38 \mathrm{E}-05$ \\
\hline $\begin{array}{l}\text { ENSXETG00000 } \\
008000\end{array}$ & $h n f 1 b$ & 4.17 & $3.22 \mathrm{E}-05$ \\
\hline $\begin{array}{l}\text { ENSXETG00000 } \\
013607\end{array}$ & axin2 & 4.05 & 3.35E-06 \\
\hline $\begin{array}{l}\text { ENSXETG00000 } \\
012490\end{array}$ & gast & 3.95 & 5.28E-05 \\
\hline $\begin{array}{l}\text { ENSXETG00000 } \\
033673\end{array}$ & fam155b & 3.81 & $1.29 \mathrm{E}-10$ \\
\hline $\begin{array}{l}\text { ENSXETG00000 } \\
026355\end{array}$ & akap12 & 3.75 & $6.41 \mathrm{E}-06$ \\
\hline $\begin{array}{l}\text { ENSXETG00000 } \\
022636\end{array}$ & prss35 & 3.65 & $2.78 \mathrm{E}-11$ \\
\hline $\begin{array}{l}\text { ENSXETG00000 } \\
006226\end{array}$ & gli1.2 & 3.65 & 2.64E-07 \\
\hline $\begin{array}{l}\text { ENSXETG00000 } \\
018365\end{array}$ & kremen2 & 3.63 & 8.94E-21 \\
\hline $\begin{array}{l}\text { ENSXETG00000 } \\
001879\end{array}$ & $n r 3 c 1$ & 3.62 & $7.96 \mathrm{E}-25$ \\
\hline $\begin{array}{l}\text { ENSXETG00000 } \\
021987\end{array}$ & hoxb6 & 3.61 & 7.21E-04 \\
\hline $\begin{array}{l}\text { ENSXETG00000 } \\
018386\end{array}$ & pou4f1.2 & 3.59 & $1.26 \mathrm{E}-05$ \\
\hline $\begin{array}{l}\text { ENSXETG00000 } \\
027510\end{array}$ & itgb6 & 3.58 & 4.61E-04 \\
\hline $\begin{array}{l}\text { ENSXETG00000 } \\
007794\end{array}$ & cldn3 & 3.54 & $1.09 \mathrm{E}-15$ \\
\hline $\begin{array}{l}\text { ENSXETG00000 } \\
000720\end{array}$ & hoxa5 & 3.53 & 3.35E-06 \\
\hline $\begin{array}{l}\text { ENSXETG00000 } \\
024347\end{array}$ & znf703 & 3.52 & 1.96E-22 \\
\hline $\begin{array}{l}\text { ENSXETG00000 } \\
010120\end{array}$ & $m p z / 2$ & 3.51 & 6.06E-12 \\
\hline $\begin{array}{l}\text { ENSXETG00000 } \\
020467\end{array}$ & cdh11 & 3.47 & 5.49E-04 \\
\hline $\begin{array}{l}\text { ENSXETG00000 } \\
021035\end{array}$ & pdgfra & 3.44 & 3.73E-04 \\
\hline $\begin{array}{l}\text { ENSXETG00000 } \\
012821\end{array}$ & sult1a1 & 3.31 & 5.55E-05 \\
\hline $\begin{array}{l}\text { ENSXETG00000 } \\
001223\end{array}$ & emilin1 & 3.30 & 1.91E-06 \\
\hline $\begin{array}{l}\text { ENSXETG00000 } \\
010128\end{array}$ & nefm & 3.29 & 7.76E-10 \\
\hline $\begin{array}{l}\text { ENSXETG00000 } \\
018864\end{array}$ & zic2 & 3.23 & $2.39 \mathrm{E}-08$ \\
\hline
\end{tabular}

\begin{tabular}{|c|c|c|c|}
\hline $\begin{array}{l}\text { ENSXETG00000 } \\
032462\end{array}$ & smpx & -2.27 & $6.19 \mathrm{E}-13$ \\
\hline $\begin{array}{l}\text { ENSXETG00000 } \\
017916\end{array}$ & galntl1 & -2.27 & 2.27E-04 \\
\hline $\begin{array}{l}\text { ENSXETG00000 } \\
012021\end{array}$ & sema $5 b$ & -2.25 & $1.99 \mathrm{E}-06$ \\
\hline $\begin{array}{l}\text { ENSXETG00000 } \\
031621\end{array}$ & c2orf70 & -2.23 & $5.25 \mathrm{E}-08$ \\
\hline $\begin{array}{l}\text { ENSXETG00000 } \\
009727\end{array}$ & prkag1 & -2.22 & 1.83E-22 \\
\hline $\begin{array}{l}\text { ENSXETG00000 } \\
013371\end{array}$ & plcb2 & -2.21 & $1.79 \mathrm{E}-02$ \\
\hline $\begin{array}{l}\text { ENSXETG00000 } \\
000901\end{array}$ & & -2.19 & $4.18 \mathrm{E}-13$ \\
\hline $\begin{array}{l}\text { ENSXETG00000 } \\
005160\end{array}$ & march3 & -2.14 & $6.21 \mathrm{E}-04$ \\
\hline $\begin{array}{l}\text { ENSXETG00000 } \\
000594\end{array}$ & foxc1 & -2.12 & $3.35 \mathrm{E}-02$ \\
\hline $\begin{array}{l}\text { ENSXETG00000 } \\
024302\end{array}$ & atp10a & -2.12 & $6.81 \mathrm{E}-03$ \\
\hline $\begin{array}{l}\text { ENSXETG00000 } \\
001696\end{array}$ & syt6 & -2.11 & 2.31E-02 \\
\hline $\begin{array}{l}\text { ENSXETG00000 } \\
015108\end{array}$ & $r d h 7$ & -2.11 & 1.07E-03 \\
\hline $\begin{array}{l}\text { ENSXETG00000 } \\
022840\end{array}$ & Iphn1 & -2.11 & $5.03 E-03$ \\
\hline $\begin{array}{l}\text { ENSXETG00000 } \\
018291\end{array}$ & cebpa & -2.10 & 7.44E-05 \\
\hline $\begin{array}{l}\text { ENSXETG00000 } \\
016618\end{array}$ & $n g f$ & -2.07 & 4.71E-04 \\
\hline $\begin{array}{l}\text { ENSXETG00000 } \\
019537\end{array}$ & $t b c 1 d 24.2$ & -2.07 & 4.56E-28 \\
\hline $\begin{array}{l}\text { ENSXETG00000 } \\
014344\end{array}$ & celf5 & -2.07 & $3.82 \mathrm{E}-03$ \\
\hline $\begin{array}{l}\text { ENSXETG00000 } \\
008779\end{array}$ & fbn1 & -2.06 & $3.11 \mathrm{E}-03$ \\
\hline $\begin{array}{l}\text { ENSXETG00000 } \\
030605\end{array}$ & loxhd1 & -2.05 & 7.17E-04 \\
\hline $\begin{array}{l}\text { ENSXETG00000 } \\
018430\end{array}$ & $n k \times 2-3$ & -2.05 & $6.22 \mathrm{E}-07$ \\
\hline $\begin{array}{l}\text { ENSXETG00000 } \\
033418\end{array}$ & & -2.05 & $2.11 \mathrm{E}-03$ \\
\hline $\begin{array}{l}\text { ENSXETG00000 } \\
015427\end{array}$ & adra2c & -2.04 & $6.14 \mathrm{E}-04$ \\
\hline $\begin{array}{l}\text { ENSXETG00000 } \\
031925\end{array}$ & & -2.04 & 3.00E-02 \\
\hline $\begin{array}{l}\text { ENSXETG00000 } \\
009502\end{array}$ & & -2.03 & 4.01E-04 \\
\hline $\begin{array}{l}\text { ENSXETG00000 } \\
001273\end{array}$ & psd4 & -2.02 & 2.70E-02 \\
\hline $\begin{array}{l}\text { ENSXETG00000 } \\
001035\end{array}$ & fam132a & -2.02 & 4.56E-03 \\
\hline $\begin{array}{l}\text { ENSXETG00000 } \\
011726\end{array}$ & ntrk2 & -2.01 & $3.78 \mathrm{E}-02$ \\
\hline $\begin{array}{l}\text { ENSXETG00000 } \\
007316\end{array}$ & s/c38a3 & -2.01 & $3.70 \mathrm{E}-13$ \\
\hline $\begin{array}{l}\text { ENSXETG00000 } \\
021301\end{array}$ & $a k d 1$ & -2.00 & $5.28 \mathrm{E}-03$ \\
\hline $\begin{array}{l}\text { ENSXETG00000 } \\
015542\end{array}$ & $\operatorname{ccdc} 78$ & -1.98 & 1.24E-07 \\
\hline $\begin{array}{l}\text { ENSXETG00000 } \\
030611\end{array}$ & Irrd1 & -1.98 & $5.30 \mathrm{E}-10$ \\
\hline $\begin{array}{l}\text { ENSXETG00000 } \\
033531\end{array}$ & tsc $22 d 4$ & -1.97 & 3.94E-02 \\
\hline $\begin{array}{l}\text { ENSXETG00000 } \\
010505\end{array}$ & phox2a & -1.96 & 3.61E-03 \\
\hline $\begin{array}{l}\text { ENSXETG00000 } \\
021999\end{array}$ & $a k 1$ & -1.96 & $1.20 \mathrm{E}-10$ \\
\hline $\begin{array}{l}\text { ENSXETG00000 } \\
017952\end{array}$ & efcab5 & -1.96 & 3.56E-09 \\
\hline $\begin{array}{l}\text { ENSXETG00000 } \\
005731\end{array}$ & sstr5 & -1.96 & 1.53E-09 \\
\hline $\begin{array}{l}\text { ENSXETG00000 } \\
017078\end{array}$ & $\mathrm{~s} 100 \mathrm{z}$ & -1.96 & $3.11 \mathrm{E}-02$ \\
\hline
\end{tabular}




\begin{tabular}{|c|c|c|c|}
\hline $\begin{array}{l}\text { ENSXETG00000 } \\
008971\end{array}$ & ywhag & 3.23 & $2.53 \mathrm{E}-10$ \\
\hline $\begin{array}{l}\text { ENSXETG00000 } \\
001974\end{array}$ & st3gal5 & 3.19 & $3.14 \mathrm{E}-07$ \\
\hline $\begin{array}{l}\text { ENSXETG00000 } \\
025936\end{array}$ & cnrip1 & 3.18 & $3.59 \mathrm{E}-03$ \\
\hline $\begin{array}{l}\text { ENSXETG00000 } \\
031488\end{array}$ & pcdh19 & 3.18 & 2.69E-04 \\
\hline $\begin{array}{l}\text { ENSXETG00000 } \\
010435\end{array}$ & prss23 & 3.17 & 4.14E-07 \\
\hline $\begin{array}{l}\text { ENSXETG00000 } \\
019101\end{array}$ & nova2 & 3.11 & $3.60 \mathrm{E}-06$ \\
\hline $\begin{array}{l}\text { ENSXETG00000 } \\
003251\end{array}$ & $\operatorname{limd} 2$ & 3.11 & $1.26 \mathrm{E}-11$ \\
\hline $\begin{array}{l}\text { ENSXETG00000 } \\
033139\end{array}$ & $z b t b 16$ & 3.09 & 5.77E-05 \\
\hline $\begin{array}{l}\text { ENSXETG00000 } \\
006965\end{array}$ & $p x d n$ & 3.06 & 4.16E-41 \\
\hline $\begin{array}{l}\text { ENSXETG00000 } \\
023975\end{array}$ & & 3.06 & $2.18 \mathrm{E}-05$ \\
\hline $\begin{array}{l}\text { ENSXETG00000 } \\
023080\end{array}$ & kif26a & 3.04 & $2.71 \mathrm{E}-04$ \\
\hline $\begin{array}{l}\text { ENSXETG00000 } \\
034301\end{array}$ & & 3.00 & $1.08 \mathrm{E}-07$ \\
\hline $\begin{array}{l}\text { ENSXETG00000 } \\
016174\end{array}$ & & 2.99 & 1.63E-06 \\
\hline $\begin{array}{l}\text { ENSXETG00000 } \\
001613\end{array}$ & emilin2 & 2.97 & 4.94E-03 \\
\hline $\begin{array}{l}\text { ENSXETG00000 } \\
022798\end{array}$ & sostdc1 & 2.95 & 7.83E-05 \\
\hline $\begin{array}{l}\text { ENSXETG00000 } \\
003565\end{array}$ & foxf1 & 2.93 & 5.36E-04 \\
\hline $\begin{array}{l}\text { ENSXETG00000 } \\
026058\end{array}$ & cnfn.1 & 2.91 & 3.30E-06 \\
\hline $\begin{array}{l}\text { ENSXETG00000 } \\
002879\end{array}$ & tspan4 & 2.89 & 2.01E-04 \\
\hline $\begin{array}{l}\text { ENSXETG00000 } \\
019550\end{array}$ & pdgfc & 2.87 & 3.38E-03 \\
\hline $\begin{array}{l}\text { ENSXETG00000 } \\
012556\end{array}$ & itgb4 & 2.84 & 9.95E-05 \\
\hline $\begin{array}{l}\text { ENSXETG00000 } \\
009114\end{array}$ & ucma & 2.82 & 2.17E-08 \\
\hline $\begin{array}{l}\text { ENSXETG00000 } \\
021674\end{array}$ & tfap2b & 2.81 & 2.45E-05 \\
\hline $\begin{array}{l}\text { ENSXETG00000 } \\
007633\end{array}$ & slco3a1 & 2.81 & 1.87E-03 \\
\hline $\begin{array}{l}\text { ENSXETG00000 } \\
018715\end{array}$ & olfm4 & 2.79 & 1.19E-08 \\
\hline $\begin{array}{l}\text { ENSXETG00000 } \\
034215\end{array}$ & sipa1 & 2.77 & 3.31E-02 \\
\hline $\begin{array}{l}\text { ENSXETG00000 } \\
014867\end{array}$ & pltp & 2.75 & $2.41 \mathrm{E}-06$ \\
\hline $\begin{array}{l}\text { ENSXETG00000 } \\
021993\end{array}$ & hoxb8 & 2.72 & 1.36E-02 \\
\hline $\begin{array}{l}\text { ENSXETG00000 } \\
010058\end{array}$ & xarp & 2.68 & $5.91 \mathrm{E}-32$ \\
\hline $\begin{array}{l}\text { ENSXETG00000 } \\
015096\end{array}$ & snap25 & 2.64 & 2.05E-04 \\
\hline $\begin{array}{l}\text { ENSXETG00000 } \\
008088\end{array}$ & & 2.63 & 4.42E-06 \\
\hline $\begin{array}{l}\text { ENSXETG00000 } \\
013886\end{array}$ & ngef & 2.62 & 1.23E-11 \\
\hline $\begin{array}{l}\text { ENSXETG00000 } \\
016741\end{array}$ & elavl3 & 2.57 & 5.94E-03 \\
\hline $\begin{array}{l}\text { ENSXETG00000 } \\
027601\end{array}$ & mex3b & 2.54 & 1.06E-07 \\
\hline $\begin{array}{l}\text { ENSXETG00000 } \\
015467\end{array}$ & zic1 & 2.54 & 7.17E-07 \\
\hline $\begin{array}{l}\text { ENSXETG00000 } \\
003871\end{array}$ & thrb & 2.53 & $3.27 \mathrm{E}-03$ \\
\hline $\begin{array}{l}\text { ENSXETG00000 } \\
006797\end{array}$ & prickle1 & 2.52 & 6.10E-15 \\
\hline $\begin{array}{l}\text { ENSXETG00000 } \\
007330\end{array}$ & hyal2 & 2.50 & $2.99 \mathrm{E}-12$ \\
\hline
\end{tabular}

\begin{tabular}{|c|c|c|c|}
\hline $\begin{array}{l}\text { ENSXETG00000 } \\
015694\end{array}$ & kcnrg & -1.95 & 2.45E-05 \\
\hline $\begin{array}{l}\text { ENSXETG00000 } \\
014625\end{array}$ & nell2 & -1.95 & 3.86E-02 \\
\hline $\begin{array}{l}\text { ENSXETG00000 } \\
014639\end{array}$ & palm & -1.95 & $1.59 \mathrm{E}-02$ \\
\hline $\begin{array}{l}\text { ENSXETG00000 } \\
021296\end{array}$ & ppil6 & -1.95 & $2.95 \mathrm{E}-06$ \\
\hline $\begin{array}{l}\text { ENSXETG00000 } \\
008426\end{array}$ & ehbp1/1 & -1.94 & 7.66E-06 \\
\hline $\begin{array}{l}\text { ENSXETG00000 } \\
031406\end{array}$ & $\ln x 2$ & -1.94 & 1.53E-02 \\
\hline $\begin{array}{l}\text { ENSXETG00000 } \\
017940\end{array}$ & c11orf41 & -1.94 & 3.93E-04 \\
\hline $\begin{array}{l}\text { ENSXETG00000 } \\
031483\end{array}$ & dnah6 & -1.94 & 2.94E-08 \\
\hline $\begin{array}{l}\text { ENSXETG00000 } \\
005172\end{array}$ & ccdc33 & -1.93 & $2.59 \mathrm{E}-10$ \\
\hline $\begin{array}{l}\text { ENSXETG00000 } \\
010345\end{array}$ & stx1a & -1.93 & 7.13E-03 \\
\hline $\begin{array}{l}\text { ENSXETG00000 } \\
010434\end{array}$ & $f z d 4$ & -1.92 & $1.89 \mathrm{E}-03$ \\
\hline $\begin{array}{l}\text { ENSXETG00000 } \\
004621\end{array}$ & tnni2 & -1.92 & $8.08 \mathrm{E}-10$ \\
\hline $\begin{array}{l}\text { ENSXETG00000 } \\
007237\end{array}$ & zfyve28 & -1.91 & $2.55 \mathrm{E}-10$ \\
\hline $\begin{array}{l}\text { ENSXETG00000 } \\
003267\end{array}$ & $t t l l 1$ & -1.89 & $1.08 \mathrm{E}-07$ \\
\hline $\begin{array}{l}\text { ENSXETG00000 } \\
009789\end{array}$ & adcy 6 & -1.89 & 1.67E-05 \\
\hline $\begin{array}{l}\text { ENSXETG00000 } \\
013478\end{array}$ & gas 212 & -1.88 & 4.00E-04 \\
\hline $\begin{array}{l}\text { ENSXETG00000 } \\
017418\end{array}$ & & -1.87 & $3.08 \mathrm{E}-13$ \\
\hline $\begin{array}{l}\text { ENSXETG00000 } \\
032935\end{array}$ & & -1.87 & 1.36E-02 \\
\hline $\begin{array}{l}\text { ENSXETG00000 } \\
032659\end{array}$ & ces3 & -1.86 & 3.33E-02 \\
\hline $\begin{array}{l}\text { ENSXETG00000 } \\
032002\end{array}$ & & -1.86 & 1.53E-04 \\
\hline $\begin{array}{l}\text { ENSXETG00000 } \\
025398\end{array}$ & gata3 & -1.85 & $1.01 \mathrm{E}-07$ \\
\hline $\begin{array}{l}\text { ENSXETG00000 } \\
006186\end{array}$ & ahrr & -1.85 & 2.83E-02 \\
\hline $\begin{array}{l}\text { ENSXETG00000 } \\
017275\end{array}$ & $d v / 3$ & -1.85 & $1.09 \mathrm{E}-06$ \\
\hline $\begin{array}{l}\text { ENSXETG00000 } \\
016042\end{array}$ & $\operatorname{tm} 4 s f 1$ & -1.85 & 1.30E-02 \\
\hline $\begin{array}{l}\text { ENSXETG00000 } \\
021393\end{array}$ & dnhd1 & -1.84 & $4.29 \mathrm{E}-02$ \\
\hline $\begin{array}{l}\text { ENSXETG00000 } \\
021314\end{array}$ & c6orf186 & -1.82 & 4.26E-02 \\
\hline $\begin{array}{l}\text { ENSXETG00000 } \\
000586\end{array}$ & jak2 & -1.81 & $6.06 \mathrm{E}-12$ \\
\hline $\begin{array}{l}\text { ENSXETG00000 } \\
005916\end{array}$ & msrb2 & -1.80 & $2.73 \mathrm{E}-06$ \\
\hline $\begin{array}{l}\text { ENSXETG00000 } \\
008821\end{array}$ & sorbs1 & -1.80 & $2.18 \mathrm{E}-06$ \\
\hline $\begin{array}{l}\text { ENSXETG00000 } \\
031224\end{array}$ & cdhr4 & -1.80 & $1.22 \mathrm{E}-02$ \\
\hline $\begin{array}{l}\text { ENSXETG00000 } \\
001935\end{array}$ & foxi4.1 & -1.79 & $1.74 \mathrm{E}-04$ \\
\hline $\begin{array}{l}\text { ENSXETG00000 } \\
030156\end{array}$ & $\operatorname{casc1}$ & -1.79 & $1.18 \mathrm{E}-03$ \\
\hline $\begin{array}{l}\text { ENSXETG00000 } \\
032214\end{array}$ & Irrtm3 & -1.79 & 3.70E-02 \\
\hline $\begin{array}{l}\text { ENSXETG00000 } \\
008299\end{array}$ & dnah2 & -1.78 & $5.73 \mathrm{E}-04$ \\
\hline $\begin{array}{l}\text { ENSXETG00000 } \\
033707\end{array}$ & & -1.78 & 5.48E-03 \\
\hline $\begin{array}{l}\text { ENSXETG00000 } \\
033377\end{array}$ & & -1.77 & $1.04 \mathrm{E}-04$ \\
\hline $\begin{array}{l}\text { ENSXETG00000 } \\
020893\end{array}$ & spag17 & -1.77 & $9.64 \mathrm{E}-06$ \\
\hline
\end{tabular}




\begin{tabular}{|c|c|c|c|}
\hline $\begin{array}{l}\text { ENSXETG00000 } \\
002878\end{array}$ & polr2l.2 & 2.49 & $9.62 \mathrm{E}-03$ \\
\hline $\begin{array}{l}\text { ENSXETG00000 } \\
006798\end{array}$ & $b t c$ & 2.48 & 3.04E-11 \\
\hline $\begin{array}{l}\text { ENSXETG00000 } \\
031898\end{array}$ & & 2.47 & 3.53E-04 \\
\hline $\begin{array}{l}\text { ENSXETG00000 } \\
012934\end{array}$ & bgn & 2.47 & $2.35 \mathrm{E}-03$ \\
\hline $\begin{array}{l}\text { ENSXETG00000 } \\
007804\end{array}$ & smad3 & 2.47 & 1.46E-04 \\
\hline $\begin{array}{l}\text { ENSXETG00000 } \\
018417\end{array}$ & sall1 & 2.44 & $1.74 \mathrm{E}-06$ \\
\hline $\begin{array}{l}\text { ENSXETG00000 } \\
013266\end{array}$ & s1pr1 & 2.36 & 3.04E-04 \\
\hline $\begin{array}{l}\text { ENSXETG00000 } \\
019108\end{array}$ & $\mathrm{ckm}$ & 2.34 & $2.96 \mathrm{E}-06$ \\
\hline $\begin{array}{l}\text { ENSXETG00000 } \\
003071\end{array}$ & $f b \ln 1$ & 2.32 & $6.94 \mathrm{E}-04$ \\
\hline $\begin{array}{l}\text { ENSXETG00000 } \\
010230\end{array}$ & grk5 & 2.31 & 4.36E-04 \\
\hline $\begin{array}{l}\text { ENSXETG00000 } \\
012029\end{array}$ & cass 4 & 2.31 & $3.58 \mathrm{E}-05$ \\
\hline $\begin{array}{l}\text { ENSXETG00000 } \\
022662\end{array}$ & epn1 & 2.30 & $4.54 \mathrm{E}-14$ \\
\hline $\begin{array}{l}\text { ENSXETG00000 } \\
005738\end{array}$ & rasd1 & 2.29 & 1.62E-04 \\
\hline $\begin{array}{l}\text { ENSXETG00000 } \\
027666\end{array}$ & tmeff1 & 2.27 & 2.61E-04 \\
\hline $\begin{array}{l}\text { ENSXETG00000 } \\
011508\end{array}$ & manea & 2.26 & $3.45 \mathrm{E}-03$ \\
\hline $\begin{array}{l}\text { ENSXETG00000 } \\
005370\end{array}$ & $t$ & 2.26 & 1.83E-03 \\
\hline $\begin{array}{l}\text { ENSXETG00000 } \\
014363\end{array}$ & vash2 & 2.25 & $7.98 \mathrm{E}-07$ \\
\hline $\begin{array}{l}\text { ENSXETG00000 } \\
016758\end{array}$ & $\begin{array}{l}x b \text {-gene- } \\
1002559\end{array}$ & 2.24 & 3.07E-04 \\
\hline $\begin{array}{l}\text { ENSXETG00000 } \\
021973\end{array}$ & hoxb4 & 2.23 & $2.38 \mathrm{E}-02$ \\
\hline $\begin{array}{l}\text { ENSXETG00000 } \\
018322\end{array}$ & $m c f 2$ & 2.23 & 8.30E-03 \\
\hline $\begin{array}{l}\text { ENSXETG00000 } \\
018908\end{array}$ & c13orf15 & 2.22 & 4.05E-06 \\
\hline $\begin{array}{l}\text { ENSXETG00000 } \\
004773\end{array}$ & col9a2 & 2.22 & 4.12E-06 \\
\hline $\begin{array}{l}\text { ENSXETG00000 } \\
017823\end{array}$ & plxnb1 & 2.21 & 3.77E-05 \\
\hline $\begin{array}{l}\text { ENSXETG00000 } \\
020971\end{array}$ & robo1 & 2.19 & $6.08 \mathrm{E}-10$ \\
\hline $\begin{array}{l}\text { ENSXETG00000 } \\
027351\end{array}$ & hoxb7 & 2.17 & 3.17E-02 \\
\hline $\begin{array}{l}\text { ENSXETG00000 } \\
027392\end{array}$ & sncaip & 2.17 & 7.27E-03 \\
\hline $\begin{array}{l}\text { ENSXETG00000 } \\
018304\end{array}$ & $t \mid x 3$ & 2.17 & 1.30E-02 \\
\hline $\begin{array}{l}\text { ENSXETG00000 } \\
009704\end{array}$ & tnfrsf19 & 2.14 & 9.39E-05 \\
\hline $\begin{array}{l}\text { ENSXETG00000 } \\
030035\end{array}$ & $a b c b 9$ & 2.14 & 7.94E-03 \\
\hline $\begin{array}{l}\text { ENSXETG00000 } \\
019934\end{array}$ & srrm4 & 2.13 & 1.32E-02 \\
\hline $\begin{array}{l}\text { ENSXETG00000 } \\
021450\end{array}$ & ampd1 & 2.13 & $3.28 \mathrm{E}-03$ \\
\hline $\begin{array}{l}\text { ENSXETG00000 } \\
022554\end{array}$ & $d h \times 32$ & 2.11 & 1.14E-09 \\
\hline $\begin{array}{l}\text { ENSXETG00000 } \\
017807\end{array}$ & spry2 & 2.11 & 3.93E-07 \\
\hline $\begin{array}{l}\text { ENSXETG00000 } \\
014718\end{array}$ & shb & 2.10 & 4.72E-17 \\
\hline $\begin{array}{l}\text { ENSXETG00000 } \\
022348\end{array}$ & sox 9 & 2.09 & 2.27E-04 \\
\hline $\begin{array}{l}\text { ENSXETG00000 } \\
010142\end{array}$ & nefl & 2.09 & 4.21E-06 \\
\hline $\begin{array}{l}\text { ENSXETG00000 } \\
006598\end{array}$ & $d k k 1$ & 2.08 & $4.04 \mathrm{E}-02$ \\
\hline
\end{tabular}

\begin{tabular}{|c|c|c|c|}
\hline $\begin{array}{l}\text { ENSXETG00000 } \\
024528\end{array}$ & cfap70 & -1.76 & 1.16E-07 \\
\hline $\begin{array}{l}\text { ENSXETG00000 } \\
025109\end{array}$ & ankar & -1.76 & 2.14E-07 \\
\hline $\begin{array}{l}\text { ENSXETG00000 } \\
011506\end{array}$ & $\begin{array}{l}x b \text {-gene- } \\
5857146\end{array}$ & -1.76 & $9.44 \mathrm{E}-12$ \\
\hline $\begin{array}{l}\text { ENSXETG00000 } \\
004373\end{array}$ & tekt2 & -1.75 & 1.40E-09 \\
\hline $\begin{array}{l}\text { ENSXETG00000 } \\
015719\end{array}$ & odf3 & -1.74 & 2.06E-08 \\
\hline $\begin{array}{l}\text { ENSXETG00000 } \\
030092\end{array}$ & & -1.74 & $2.28 \mathrm{E}-03$ \\
\hline $\begin{array}{l}\text { ENSXETG00000 } \\
021988\end{array}$ & $d c d c 2$ & -1.74 & 4.76E-06 \\
\hline $\begin{array}{l}\text { ENSXETG00000 } \\
024508\end{array}$ & & -1.74 & 1.42E-08 \\
\hline $\begin{array}{l}\text { ENSXETG00000 } \\
032283\end{array}$ & dnah3 & -1.73 & 8.46E-07 \\
\hline $\begin{array}{l}\text { ENSXETG00000 } \\
021518\end{array}$ & kcnn2 & -1.73 & $1.91 \mathrm{E}-03$ \\
\hline $\begin{array}{l}\text { ENSXETG00000 } \\
018025\end{array}$ & slc22a23 & -1.73 & $2.39 \mathrm{E}-04$ \\
\hline $\begin{array}{l}\text { ENSXETG00000 } \\
030029\end{array}$ & dnah1 & -1.73 & $4.20 \mathrm{E}-08$ \\
\hline $\begin{array}{l}\text { ENSXETG00000 } \\
000659\end{array}$ & efhc1 & -1.72 & 1.11E-09 \\
\hline $\begin{array}{l}\text { ENSXETG00000 } \\
031311\end{array}$ & & -1.72 & $8.25 \mathrm{E}-05$ \\
\hline $\begin{array}{l}\text { ENSXETG00000 } \\
013506\end{array}$ & rnf32 & -1.72 & $2.64 \mathrm{E}-02$ \\
\hline $\begin{array}{l}\text { ENSXETG00000 } \\
004208\end{array}$ & $r t d r 1$ & -1.71 & $1.52 \mathrm{E}-08$ \\
\hline $\begin{array}{l}\text { ENSXETG00000 } \\
031053\end{array}$ & ttc6 & -1.71 & $3.26 \mathrm{E}-05$ \\
\hline $\begin{array}{l}\text { ENSXETG00000 } \\
001388\end{array}$ & dhrs3 & -1.71 & $5.67 \mathrm{E}-07$ \\
\hline $\begin{array}{l}\text { ENSXETG00000 } \\
024630\end{array}$ & ccdc153 & -1.70 & 8.28E-03 \\
\hline $\begin{array}{l}\text { ENSXETG00000 } \\
016412\end{array}$ & rasal1 & -1.70 & 9.02E-09 \\
\hline $\begin{array}{l}\text { ENSXETG00000 } \\
023103\end{array}$ & spata18 & -1.70 & 2.05E-06 \\
\hline $\begin{array}{l}\text { ENSXETG00000 } \\
003039\end{array}$ & & -1.70 & 4.29E-03 \\
\hline $\begin{array}{l}\text { ENSXETG00000 } \\
021297\end{array}$ & ak9 & -1.69 & $1.55 \mathrm{E}-06$ \\
\hline $\begin{array}{l}\text { ENSXETG00000 } \\
004297\end{array}$ & ppp1r1a & -1.69 & $3.11 \mathrm{E}-02$ \\
\hline $\begin{array}{l}\text { ENSXETG00000 } \\
002217\end{array}$ & kiaa1841 & -1.69 & $4.85 \mathrm{E}-04$ \\
\hline $\begin{array}{l}\text { ENSXETG00000 } \\
009722\end{array}$ & ca12 & -1.68 & $5.65 \mathrm{E}-04$ \\
\hline $\begin{array}{l}\text { ENSXETG00000 } \\
015133\end{array}$ & phldb3 & -1.68 & $2.17 \mathrm{E}-03$ \\
\hline $\begin{array}{l}\text { ENSXETG00000 } \\
022889\end{array}$ & $z b b x$ & -1.68 & $2.32 \mathrm{E}-02$ \\
\hline $\begin{array}{l}\text { ENSXETG00000 } \\
006838\end{array}$ & rab37 & -1.68 & $2.58 \mathrm{E}-04$ \\
\hline $\begin{array}{l}\text { ENSXETG00000 } \\
019163\end{array}$ & $i \operatorname{tg} b 3$ & -1.68 & $1.29 \mathrm{E}-03$ \\
\hline $\begin{array}{l}\text { ENSXETG00000 } \\
007116\end{array}$ & $\operatorname{ttc} 29$ & -1.68 & 5.83E-05 \\
\hline $\begin{array}{l}\text { ENSXETG00000 } \\
014624\end{array}$ & gmpr2 & -1.68 & $9.38 \mathrm{E}-13$ \\
\hline $\begin{array}{l}\text { ENSXETG00000 } \\
032601\end{array}$ & akna & -1.68 & 4.08E-02 \\
\hline $\begin{array}{l}\text { ENSXETG00000 } \\
003663\end{array}$ & pcdh10 & -1.67 & $6.34 \mathrm{E}-03$ \\
\hline $\begin{array}{l}\text { ENSXETG00000 } \\
016964\end{array}$ & $b t b d 11$ & -1.67 & 1.39E-02 \\
\hline $\begin{array}{l}\text { ENSXETG00000 } \\
001718\end{array}$ & & -1.67 & $1.99 \mathrm{E}-02$ \\
\hline $\begin{array}{l}\text { ENSXETG00000 } \\
006261\end{array}$ & c9orf9 & -1.67 & $1.60 \mathrm{E}-05$ \\
\hline
\end{tabular}




\begin{tabular}{|c|c|c|c|c|c|c|c|}
\hline $\begin{array}{l}\text { ENSXETG00000 } \\
011173\end{array}$ & wnt10b & 2.07 & 4.97E-02 & $\begin{array}{l}\text { ENSXETG00000 } \\
023392\end{array}$ & rassf6 & -1.67 & $7.65 \mathrm{E}-05$ \\
\hline $\begin{array}{l}\text { ENSXETG00000 } \\
025522\end{array}$ & esr10 & 2.06 & $2.47 \mathrm{E}-02$ & $\begin{array}{l}\text { ENSXETG00000 } \\
026100\end{array}$ & c5orf42 & -1.67 & $5.28 \mathrm{E}-07$ \\
\hline $\begin{array}{l}\text { ENSXETG00000 } \\
003296\end{array}$ & cxcr7 & 2.06 & $1.26 \mathrm{E}-05$ & $\begin{array}{l}\text { ENSXETG00000 } \\
030387\end{array}$ & c3orf84 & -1.67 & $1.88 \mathrm{E}-02$ \\
\hline $\begin{array}{l}\text { ENSXETG00000 } \\
003938\end{array}$ & robo3 & 2.06 & $5.52 \mathrm{E}-23$ & $\begin{array}{l}\text { ENSXETG00000 } \\
018416\end{array}$ & clcnkb & -1.67 & 1.16E-02 \\
\hline $\begin{array}{l}\text { ENSXETG00000 } \\
031424\end{array}$ & & 2.05 & 3.76E-03 & $\begin{array}{l}\text { ENSXETG00000 } \\
013869\end{array}$ & ccdc108 & -1.66 & 1.06E-09 \\
\hline $\begin{array}{l}\text { ENSXETG00000 } \\
013228\end{array}$ & egflam & 2.04 & $2.21 \mathrm{E}-04$ & $\begin{array}{l}\text { ENSXETG00000 } \\
013373\end{array}$ & fam81b & -1.65 & 1.33E-04 \\
\hline $\begin{array}{l}\text { ENSXETG00000 } \\
016415\end{array}$ & gas $2 / 1$ & 2.04 & 1.59E-04 & $\begin{array}{l}\text { ENSXETG00000 } \\
018707\end{array}$ & capsl & -1.65 & $2.04 \mathrm{E}-03$ \\
\hline $\begin{array}{l}\text { ENSXETG00000 } \\
022245\end{array}$ & hes9.1 & 2.04 & 4.43E-02 & $\begin{array}{l}\text { ENSXETG00000 } \\
016146\end{array}$ & $\sec 14 / 3$ & -1.65 & $1.81 \mathrm{E}-07$ \\
\hline $\begin{array}{l}\text { ENSXETG00000 } \\
005880\end{array}$ & hip1 & 2.02 & $2.18 \mathrm{E}-03$ & $\begin{array}{l}\text { ENSXETG00000 } \\
016844\end{array}$ & wdr38 & -1.65 & $2.47 \mathrm{E}-06$ \\
\hline $\begin{array}{l}\text { ENSXETG00000 } \\
001058\end{array}$ & klf17 & 2.02 & $3.99 \mathrm{E}-08$ & $\begin{array}{l}\text { ENSXETG00000 } \\
019005\end{array}$ & & -1.65 & $2.70 \mathrm{E}-05$ \\
\hline $\begin{array}{l}\text { ENSXETG00000 } \\
023193\end{array}$ & p2ry 4 & 1.99 & $2.23 \mathrm{E}-10$ & $\begin{array}{l}\text { ENSXETG00000 } \\
006227\end{array}$ & arhgef25 & -1.64 & 3.33E-02 \\
\hline $\begin{array}{l}\text { ENSXETG00000 } \\
017641\end{array}$ & $\begin{array}{l}x b \text {-gene- } \\
5781886\end{array}$ & 1.98 & 8.76E-05 & $\begin{array}{l}\text { ENSXETG00000 } \\
010988\end{array}$ & ndrg1 & -1.64 & 3.87E-02 \\
\hline $\begin{array}{l}\text { ENSXETG00000 } \\
000377\end{array}$ & fras 1 & 1.95 & $2.49 \mathrm{E}-03$ & $\begin{array}{l}\text { ENSXETG00000 } \\
008688\end{array}$ & & -1.64 & $3.89 \mathrm{E}-08$ \\
\hline $\begin{array}{l}\text { ENSXETG00000 } \\
004222\end{array}$ & adora2a & 1.94 & 1.06E-03 & $\begin{array}{l}\text { ENSXETG00000 } \\
009174\end{array}$ & c9orf21 & -1.64 & 1.13E-03 \\
\hline $\begin{array}{l}\text { ENSXETG00000 } \\
007241\end{array}$ & $d b n 1$ & 1.94 & $1.62 \mathrm{E}-03$ & $\begin{array}{l}\text { ENSXETG00000 } \\
012367\end{array}$ & syne1 & -1.63 & $4.50 \mathrm{E}-07$ \\
\hline $\begin{array}{l}\text { ENSXETG00000 } \\
010865\end{array}$ & aldh1a2 & 1.94 & $2.14 \mathrm{E}-03$ & $\begin{array}{l}\text { ENSXETG00000 } \\
000658\end{array}$ & paqr8 & -1.63 & $3.61 \mathrm{E}-02$ \\
\hline $\begin{array}{l}\text { ENSXETG00000 } \\
008399\end{array}$ & flt4 & 1.94 & $2.75 \mathrm{E}-02$ & $\begin{array}{l}\text { ENSXETG00000 } \\
024641\end{array}$ & elovl2 & -1.63 & 2.69E-07 \\
\hline $\begin{array}{l}\text { ENSXETG00000 } \\
015816\end{array}$ & blvra & 1.94 & $2.83 \mathrm{E}-06$ & $\begin{array}{l}\text { ENSXETG00000 } \\
027678\end{array}$ & gnaz & -1.63 & 1.16E-07 \\
\hline $\begin{array}{l}\text { ENSXETG00000 } \\
021140\end{array}$ & cyp1a1 & 1.92 & 4.73E-02 & $\begin{array}{l}\text { ENSXETG00000 } \\
011269\end{array}$ & cdh15 & -1.63 & $6.04 \mathrm{E}-04$ \\
\hline $\begin{array}{l}\text { ENSXETG00000 } \\
012279\end{array}$ & lamb1 & 1.90 & $2.28 \mathrm{E}-05$ & $\begin{array}{l}\text { ENSXETG00000 } \\
004835\end{array}$ & efhc2 & -1.63 & 7.34E-05 \\
\hline $\begin{array}{l}\text { ENSXETG00000 } \\
029924\end{array}$ & stxbp5 & 1.90 & 8.96E-11 & $\begin{array}{l}\text { ENSXETG00000 } \\
017994\end{array}$ & pkhd1/1 & -1.63 & $3.27 \mathrm{E}-02$ \\
\hline $\begin{array}{l}\text { ENSXETG00000 } \\
030428\end{array}$ & & 1.90 & 3.35E-02 & $\begin{array}{l}\text { ENSXETG00000 } \\
019512\end{array}$ & pik3r6 & -1.62 & 1.43E-02 \\
\hline $\begin{array}{l}\text { ENSXETG00000 } \\
012216\end{array}$ & msi1 & 1.88 & 2.56E-07 & $\begin{array}{l}\text { ENSXETG00000 } \\
005336\end{array}$ & prr29 & -1.62 & $9.81 \mathrm{E}-05$ \\
\hline $\begin{array}{l}\text { ENSXETG00000 } \\
010662\end{array}$ & ap3b2 & 1.88 & 1.06E-02 & $\begin{array}{l}\text { ENSXETG00000 } \\
022781\end{array}$ & ets1 & -1.62 & 4.57E-04 \\
\hline $\begin{array}{l}\text { ENSXETG00000 } \\
019579\end{array}$ & pmp22 & 1.88 & 1.57E-02 & $\begin{array}{l}\text { ENSXETG00000 } \\
015189\end{array}$ & c2orf73 & -1.62 & $5.92 \mathrm{E}-06$ \\
\hline $\begin{array}{l}\text { ENSXETG00000 } \\
001066\end{array}$ & gpr63 & 1.88 & $4.97 \mathrm{E}-02$ & $\begin{array}{l}\text { ENSXETG00000 } \\
003686\end{array}$ & als2cl & -1.62 & $1.60 \mathrm{E}-09$ \\
\hline $\begin{array}{l}\text { ENSXETG00000 } \\
032528\end{array}$ & sspo & 1.87 & $4.28 \mathrm{E}-02$ & $\begin{array}{l}\text { ENSXETG00000 } \\
018553\end{array}$ & dnah14 & -1.62 & $1.61 \mathrm{E}-04$ \\
\hline $\begin{array}{l}\text { ENSXETG00000 } \\
000758\end{array}$ & pcsk5 & 1.87 & $1.18 \mathrm{E}-06$ & $\begin{array}{l}\text { ENSXETG00000 } \\
013834\end{array}$ & c17orf105 & -1.61 & 7.94E-04 \\
\hline $\begin{array}{l}\text { ENSXETG00000 } \\
005259\end{array}$ & stk40 & 1.86 & 1.36E-04 & $\begin{array}{l}\text { ENSXETG00000 } \\
014590\end{array}$ & ccdc170 & -1.61 & 3.51E-09 \\
\hline $\begin{array}{l}\text { ENSXETG00000 } \\
019568\end{array}$ & spry4 & 1.86 & $3.22 \mathrm{E}-03$ & $\begin{array}{l}\text { ENSXETG00000 } \\
010527\end{array}$ & rsph1 & -1.61 & 1.43E-09 \\
\hline $\begin{array}{l}\text { ENSXETG00000 } \\
009686\end{array}$ & insm1 & 1.85 & $1.22 \mathrm{E}-02$ & $\begin{array}{l}\text { ENSXETG00000 } \\
033037\end{array}$ & & -1.61 & $2.12 \mathrm{E}-07$ \\
\hline $\begin{array}{l}\text { ENSXETG00000 } \\
015431\end{array}$ & mylip & 1.85 & $1.00 \mathrm{E}-04$ & $\begin{array}{l}\text { ENSXETG00000 } \\
032089\end{array}$ & spef1 & -1.61 & 3.57E-05 \\
\hline $\begin{array}{l}\text { ENSXETG00000 } \\
029928\end{array}$ & gas1 & 1.85 & $3.30 \mathrm{E}-06$ & $\begin{array}{l}\text { ENSXETG00000 } \\
007897\end{array}$ & h2afy & -1.61 & $2.08 \mathrm{E}-03$ \\
\hline $\begin{array}{l}\text { ENSXETG00000 } \\
018847\end{array}$ & plk2 & 1.83 & $7.47 \mathrm{E}-16$ & $\begin{array}{l}\text { ENSXETG00000 } \\
008812\end{array}$ & $f z d 8$ & -1.61 & 4.77E-04 \\
\hline $\begin{array}{l}\text { ENSXETG00000 } \\
008674\end{array}$ & prdm1 & 1.83 & 1.55E-05 & $\begin{array}{l}\text { ENSXETG00000 } \\
006725\end{array}$ & c11orf66 & -1.60 & $2.42 \mathrm{E}-06$ \\
\hline $\begin{array}{l}\text { ENSXETG00000 } \\
030115\end{array}$ & $s p 8$ & 1.81 & $2.17 \mathrm{E}-08$ & $\begin{array}{l}\text { ENSXETG00000 } \\
005595\end{array}$ & tns1 & -1.60 & 1.30E-02 \\
\hline $\begin{array}{l}\text { ENSXETG00000 } \\
016853\end{array}$ & $s / c 23 a 2$ & 1.80 & 8.61E-05 & $\begin{array}{l}\text { ENSXETG00000 } \\
007384\end{array}$ & spag6 & -1.60 & $2.40 \mathrm{E}-11$ \\
\hline
\end{tabular}




\begin{tabular}{|c|c|c|c|c|c|c|c|}
\hline $\begin{array}{l}\text { ENSXETG00000 } \\
021344\end{array}$ & fyn & 1.79 & $4.12 \mathrm{E}-02$ & $\begin{array}{l}\text { ENSXETG00000 } \\
026587\end{array}$ & ccdc180 & -1.60 & 7.03E-05 \\
\hline $\begin{array}{l}\text { ENSXETG00000 } \\
017665\end{array}$ & Ihx $x$ & 1.78 & $3.52 \mathrm{E}-02$ & $\begin{array}{l}\text { ENSXETG00000 } \\
030126\end{array}$ & & -1.60 & $1.53 \mathrm{E}-06$ \\
\hline $\begin{array}{l}\text { ENSXETG00000 } \\
016286\end{array}$ & $\operatorname{trps} 1$ & 1.77 & 1.89E-03 & $\begin{array}{l}\text { ENSXETG00000 } \\
023295\end{array}$ & Imo4.1 & -1.60 & $2.98 \mathrm{E}-08$ \\
\hline $\begin{array}{l}\text { ENSXETG00000 } \\
002103\end{array}$ & $n d n f$ & 1.76 & $2.45 \mathrm{E}-02$ & $\begin{array}{l}\text { ENSXETG00000 } \\
005917\end{array}$ & armc3 & -1.60 & 1.04E-06 \\
\hline $\begin{array}{l}\text { ENSXETG00000 } \\
015557\end{array}$ & vsnl1 & 1.76 & 1.43E-09 & $\begin{array}{l}\text { ENSXETG00000 } \\
010437\end{array}$ & ccdc81 & -1.59 & $1.59 \mathrm{E}-04$ \\
\hline $\begin{array}{l}\text { ENSXETG00000 } \\
007560\end{array}$ & elf5 & 1.76 & 4.11E-02 & $\begin{array}{l}\text { ENSXETG00000 } \\
008630\end{array}$ & wdr63 & -1.59 & $1.38 \mathrm{E}-08$ \\
\hline $\begin{array}{l}\text { ENSXETG00000 } \\
022354\end{array}$ & nos 1 & 1.76 & $3.36 \mathrm{E}-02$ & $\begin{array}{l}\text { ENSXETG00000 } \\
022691\end{array}$ & dzip1I & -1.59 & $2.18 \mathrm{E}-06$ \\
\hline $\begin{array}{l}\text { ENSXETG00000 } \\
011941\end{array}$ & tmem132a & 1.75 & 7.19E-04 & $\begin{array}{l}\text { ENSXETG00000 } \\
016326\end{array}$ & morn5 & -1.59 & $8.25 \mathrm{E}-05$ \\
\hline $\begin{array}{l}\text { ENSXETG00000 } \\
014993\end{array}$ & $k c p$ & 1.75 & 4.64E-02 & $\begin{array}{l}\text { ENSXETG00000 } \\
030661\end{array}$ & & -1.59 & $1.16 \mathrm{E}-03$ \\
\hline $\begin{array}{l}\text { ENSXETG00000 } \\
003934\end{array}$ & slc37a2 & 1.74 & $2.81 \mathrm{E}-09$ & $\begin{array}{l}\text { ENSXETG00000 } \\
000514\end{array}$ & mgat5 & -1.58 & $6.29 \mathrm{E}-03$ \\
\hline $\begin{array}{l}\text { ENSXETG00000 } \\
010956\end{array}$ & mycl1 & 1.73 & 1.62E-04 & $\begin{array}{l}\text { ENSXETG00000 } \\
014934\end{array}$ & c2orf62 & -1.58 & $2.27 \mathrm{E}-05$ \\
\hline $\begin{array}{l}\text { ENSXETG00000 } \\
002931\end{array}$ & $f z d 10$ & 1.73 & $2.78 \mathrm{E}-10$ & $\begin{array}{l}\text { ENSXETG00000 } \\
034198\end{array}$ & & -1.58 & 4.56E-02 \\
\hline $\begin{array}{l}\text { ENSXETG00000 } \\
023872\end{array}$ & ngfr & 1.72 & $2.74 \mathrm{E}-07$ & $\begin{array}{l}\text { ENSXETG00000 } \\
016481\end{array}$ & gjb3 & -1.58 & 8.65E-03 \\
\hline $\begin{array}{l}\text { ENSXETG00000 } \\
023832\end{array}$ & tmcc3 & 1.71 & $8.11 \mathrm{E}-04$ & $\begin{array}{l}\text { ENSXETG00000 } \\
008519\end{array}$ & add2 & -1.58 & $5.20 \mathrm{E}-03$ \\
\hline $\begin{array}{l}\text { ENSXETG00000 } \\
000251\end{array}$ & hmox1 & 1.71 & 1.23E-07 & $\begin{array}{l}\text { ENSXETG00000 } \\
022961\end{array}$ & kcnj16 & -1.58 & 4.91E-03 \\
\hline $\begin{array}{l}\text { ENSXETG00000 } \\
005911\end{array}$ & hes6.1 & 1.69 & $5.58 \mathrm{E}-05$ & $\begin{array}{l}\text { ENSXETG00000 } \\
032300\end{array}$ & stmnd1 & -1.58 & 1.79E-09 \\
\hline $\begin{array}{l}\text { ENSXETG00000 } \\
001837\end{array}$ & trim2 & 1.69 & $2.04 \mathrm{E}-03$ & $\begin{array}{l}\text { ENSXETG00000 } \\
022016\end{array}$ & ribc1 & -1.57 & 2.39E-08 \\
\hline $\begin{array}{l}\text { ENSXETG00000 } \\
016100\end{array}$ & tcf7 & 1.67 & $2.16 \mathrm{E}-06$ & $\begin{array}{l}\text { ENSXETG00000 } \\
024952\end{array}$ & tnnt3 & -1.57 & 4.02E-02 \\
\hline $\begin{array}{l}\text { ENSXETG00000 } \\
010491\end{array}$ & pcbp2 & 1.67 & 4.05E-02 & $\begin{array}{l}\text { ENSXETG00000 } \\
019324\end{array}$ & bcam & -1.57 & $1.85 \mathrm{E}-02$ \\
\hline $\begin{array}{l}\text { ENSXETG00000 } \\
001802\end{array}$ & $m t \times 2$ & 1.66 & $1.27 \mathrm{E}-03$ & $\begin{array}{l}\text { ENSXETG00000 } \\
024951\end{array}$ & stard9 & -1.57 & $4.38 \mathrm{E}-04$ \\
\hline $\begin{array}{l}\text { ENSXETG00000 } \\
010410\end{array}$ & phf15 & 1.65 & $3.72 \mathrm{E}-02$ & $\begin{array}{l}\text { ENSXETG00000 } \\
018735\end{array}$ & tcf7l2 & -1.57 & $2.47 \mathrm{E}-02$ \\
\hline $\begin{array}{l}\text { ENSXETG00000 } \\
021972\end{array}$ & col27a1 & 1.65 & $2.04 \mathrm{E}-04$ & $\begin{array}{l}\text { ENSXETG00000 } \\
008892\end{array}$ & irx4 & -1.57 & $1.66 \mathrm{E}-04$ \\
\hline $\begin{array}{l}\text { ENSXETG00000 } \\
007198\end{array}$ & nudt22 & 1.65 & $9.82 \mathrm{E}-03$ & $\begin{array}{l}\text { ENSXETG00000 } \\
015159\end{array}$ & em/6 & -1.57 & 4.61E-04 \\
\hline $\begin{array}{l}\text { ENSXETG00000 } \\
006532\end{array}$ & fbxo10 & 1.63 & $2.26 \mathrm{E}-02$ & $\begin{array}{l}\text { ENSXETG00000 } \\
020037\end{array}$ & & -1.56 & $1.14 \mathrm{E}-02$ \\
\hline $\begin{array}{l}\text { ENSXETG00000 } \\
025626\end{array}$ & tmem69 & 1.63 & 1.52E-08 & $\begin{array}{l}\text { ENSXETG00000 } \\
015289\end{array}$ & anxa2 & -1.56 & 7.39E-06 \\
\hline $\begin{array}{l}\text { ENSXETG00000 } \\
019257\end{array}$ & rab34 & 1.62 & 1.15E-02 & $\begin{array}{l}\text { ENSXETG00000 } \\
021497\end{array}$ & epb41/4a & -1.56 & $3.55 \mathrm{E}-04$ \\
\hline $\begin{array}{l}\text { ENSXETG00000 } \\
016157\end{array}$ & znf219 & 1.62 & $4.30 \mathrm{E}-02$ & $\begin{array}{l}\text { ENSXETG00000 } \\
015923\end{array}$ & $\begin{array}{l}x b-\text { gene- } \\
5949053\end{array}$ & -1.56 & 1.33E-02 \\
\hline $\begin{array}{l}\text { ENSXETG00000 } \\
023439\end{array}$ & sox11 & 1.61 & $2.18 \mathrm{E}-05$ & $\begin{array}{l}\text { ENSXETG00000 } \\
005341\end{array}$ & cxorf22 & -1.56 & $9.91 \mathrm{E}-03$ \\
\hline $\begin{array}{l}\text { ENSXETG00000 } \\
011665\end{array}$ & ncam1 & 1.61 & $2.58 \mathrm{E}-02$ & $\begin{array}{l}\text { ENSXETG00000 } \\
004292\end{array}$ & rapgef3 & -1.55 & $1.31 \mathrm{E}-03$ \\
\hline $\begin{array}{l}\text { ENSXETG00000 } \\
002340\end{array}$ & jag1 & 1.60 & 2.30E-03 & $\begin{array}{l}\text { ENSXETG00000 } \\
016804\end{array}$ & inpp5a.2 & -1.55 & $5.98 \mathrm{E}-03$ \\
\hline $\begin{array}{l}\text { ENSXETG00000 } \\
021454\end{array}$ & dennd $2 c$ & 1.60 & $2.25 \mathrm{E}-02$ & $\begin{array}{l}\text { ENSXETG00000 } \\
032500\end{array}$ & fam179a & -1.55 & 7.63E-04 \\
\hline $\begin{array}{l}\text { ENSXETG00000 } \\
013354\end{array}$ & slitrk1 & 1.60 & 1.93E-02 & $\begin{array}{l}\text { ENSXETG00000 } \\
007146\end{array}$ & Irrc18 & -1.55 & $5.24 \mathrm{E}-06$ \\
\hline $\begin{array}{l}\text { ENSXETG00000 } \\
005062\end{array}$ & etv1 & 1.60 & 1.67E-04 & $\begin{array}{l}\text { ENSXETG00000 } \\
001021\end{array}$ & асар3 & -1.55 & $4.86 \mathrm{E}-04$ \\
\hline $\begin{array}{l}\text { ENSXETG00000 } \\
022669\end{array}$ & mmp14 & 1.58 & 1.86E-05 & $\begin{array}{l}\text { ENSXETG00000 } \\
030526\end{array}$ & tsga10 & -1.55 & 5.57E-05 \\
\hline $\begin{array}{l}\text { ENSXETG00000 } \\
012404\end{array}$ & $f z d 7$ & 1.57 & 1.90E-04 & $\begin{array}{l}\text { ENSXETG00000 } \\
022934\end{array}$ & $\begin{array}{l}x b \text {-gene- } \\
5992704\end{array}$ & -1.55 & $5.46 \mathrm{E}-07$ \\
\hline $\begin{array}{l}\text { ENSXETG00000 } \\
017378\end{array}$ & ptgs 2 & 1.57 & $2.37 \mathrm{E}-05$ & $\begin{array}{l}\text { ENSXETG00000 } \\
015878\end{array}$ & hydin & -1.54 & $6.21 \mathrm{E}-07$ \\
\hline $\begin{array}{l}\text { ENSXETG00000 } \\
018892\end{array}$ & ptch2 & 1.55 & 4.19E-03 & $\begin{array}{l}\text { ENSXETG00000 } \\
011560\end{array}$ & foxp2 & -1.54 & $7.09 \mathrm{E}-03$ \\
\hline
\end{tabular}




\begin{tabular}{|c|c|c|c|c|c|c|c|}
\hline $\begin{array}{l}\text { ENSXETG00000 } \\
034300\end{array}$ & & 1.54 & $6.43 \mathrm{E}-06$ & $\begin{array}{l}\text { ENSXETG00000 } \\
024614\end{array}$ & tbcel & -1.54 & 1.43E-13 \\
\hline $\begin{array}{l}\text { ENSXETG00000 } \\
013631\end{array}$ & adamts1 & 1.53 & $6.68 \mathrm{E}-03$ & $\begin{array}{l}\text { ENSXETG00000 } \\
019733\end{array}$ & ccdc146 & -1.54 & 7.95E-08 \\
\hline $\begin{array}{l}\text { ENSXETG00000 } \\
016416\end{array}$ & ras/10a & 1.53 & 1.65E-03 & $\begin{array}{l}\text { ENSXETG00000 } \\
021596\end{array}$ & gpr146 & -1.54 & 4.01E-04 \\
\hline $\begin{array}{l}\text { ENSXETG00000 } \\
011490\end{array}$ & col6a2 & 1.52 & $1.72 \mathrm{E}-02$ & $\begin{array}{l}\text { ENSXETG00000 } \\
024914\end{array}$ & rab36 & -1.54 & 1.10E-05 \\
\hline $\begin{array}{l}\text { ENSXETG00000 } \\
005928\end{array}$ & dag1 & 1.52 & 4.70E-06 & $\begin{array}{l}\text { ENSXETG00000 } \\
023542\end{array}$ & $\begin{array}{l}x b \text {-gene- } \\
5950142\end{array}$ & -1.53 & $2.12 \mathrm{E}-04$ \\
\hline $\begin{array}{l}\text { ENSXETG00000 } \\
032223\end{array}$ & cltcl1 & 1.52 & $1.62 \mathrm{E}-03$ & $\begin{array}{l}\text { ENSXETG00000 } \\
016135\end{array}$ & hhla2 & -1.53 & $1.18 \mathrm{E}-02$ \\
\hline $\begin{array}{l}\text { ENSXETG00000 } \\
012718\end{array}$ & dpys/3 & 1.52 & $2.90 \mathrm{E}-02$ & $\begin{array}{l}\text { ENSXETG00000 } \\
008117\end{array}$ & fam63a & -1.53 & 4.31E-07 \\
\hline $\begin{array}{l}\text { ENSXETG00000 } \\
021813\end{array}$ & cttnbp2 & 1.51 & 4.42E-05 & $\begin{array}{l}\text { ENSXETG00000 } \\
019168\end{array}$ & c13orf26 & -1.53 & 2.42E-11 \\
\hline $\begin{array}{l}\text { ENSXETG00000 } \\
014353\end{array}$ & tle2 & 1.51 & 2.04E-06 & $\begin{array}{l}\text { ENSXETG00000 } \\
014319\end{array}$ & eno4 & -1.53 & 4.50E-10 \\
\hline $\begin{array}{l}\text { ENSXETG00000 } \\
010707\end{array}$ & sparc & 1.51 & $9.66 \mathrm{E}-03$ & $\begin{array}{l}\text { ENSXETG00000 } \\
012713\end{array}$ & stk33 & -1.53 & 8.82E-04 \\
\hline $\begin{array}{l}\text { ENSXETG00000 } \\
004642\end{array}$ & cdc42ep4 & 1.50 & $9.92 \mathrm{E}-05$ & $\begin{array}{l}\text { ENSXETG00000 } \\
002847\end{array}$ & c14orf45 & -1.52 & $4.61 \mathrm{E}-04$ \\
\hline $\begin{array}{l}\text { ENSXETG00000 } \\
027807\end{array}$ & cited2 & 1.50 & $3.36 \mathrm{E}-02$ & $\begin{array}{l}\text { ENSXETG00000 } \\
012703\end{array}$ & klhdc9 & -1.52 & $6.90 \mathrm{E}-03$ \\
\hline $\begin{array}{l}\text { ENSXETG00000 } \\
000904\end{array}$ & il17rd & 1.48 & $6.79 \mathrm{E}-03$ & $\begin{array}{l}\text { ENSXETG00000 } \\
027390\end{array}$ & znf474 & -1.52 & 4.49E-13 \\
\hline $\begin{array}{l}\text { ENSXETG00000 } \\
026881\end{array}$ & $n r 2 c 2 a p$ & 1.48 & $3.37 \mathrm{E}-02$ & $\begin{array}{l}\text { ENSXETG00000 } \\
033778\end{array}$ & tbata & -1.51 & $3.06 \mathrm{E}-07$ \\
\hline $\begin{array}{l}\text { ENSXETG00000 } \\
025478\end{array}$ & lum & 1.48 & $2.02 \mathrm{E}-02$ & $\begin{array}{l}\text { ENSXETG00000 } \\
030130\end{array}$ & & -1.51 & $9.46 \mathrm{E}-04$ \\
\hline $\begin{array}{l}\text { ENSXETG00000 } \\
009405\end{array}$ & $w n t 5 b$ & 1.48 & $3.23 \mathrm{E}-03$ & $\begin{array}{l}\text { ENSXETG00000 } \\
025251\end{array}$ & dnah9 & -1.51 & 2.32E-02 \\
\hline $\begin{array}{l}\text { ENSXETG00000 } \\
011877\end{array}$ & cd74 & 1.47 & $2.22 \mathrm{E}-03$ & $\begin{array}{l}\text { ENSXETG00000 } \\
033908\end{array}$ & ube $2 u$ & -1.51 & 3.92E-02 \\
\hline $\begin{array}{l}\text { ENSXETG00000 } \\
000581\end{array}$ & $\mathrm{fmn} / 3$ & 1.46 & $7.96 \mathrm{E}-04$ & $\begin{array}{l}\text { ENSXETG00000 } \\
005487\end{array}$ & arhgap42 & -1.51 & $6.21 \mathrm{E}-07$ \\
\hline $\begin{array}{l}\text { ENSXETG00000 } \\
000696\end{array}$ & rai14 & 1.46 & 2.06E-04 & $\begin{array}{l}\text { ENSXETG00000 } \\
015093\end{array}$ & ankrd5 & -1.50 & $1.54 \mathrm{E}-11$ \\
\hline $\begin{array}{l}\text { ENSXETG00000 } \\
016573\end{array}$ & $d l \times 6$ & 1.46 & 7.31E-04 & $\begin{array}{l}\text { ENSXETG00000 } \\
022383\end{array}$ & mapk4 & -1.50 & 3.43E-02 \\
\hline $\begin{array}{l}\text { ENSXETG00000 } \\
023744\end{array}$ & ptp4a3 & 1.45 & 7.14E-04 & $\begin{array}{l}\text { ENSXETG00000 } \\
030188\end{array}$ & dnah10 & -1.50 & 2.63E-06 \\
\hline $\begin{array}{l}\text { ENSXETG00000 } \\
031265\end{array}$ & raph1 & 1.45 & $9.02 \mathrm{E}-05$ & $\begin{array}{l}\text { ENSXETG00000 } \\
019423\end{array}$ & plcg1 & -1.49 & 7.63E-04 \\
\hline $\begin{array}{l}\text { ENSXETG00000 } \\
006108\end{array}$ & col9a3 & 1.45 & $9.54 \mathrm{E}-04$ & $\begin{array}{l}\text { ENSXETG00000 } \\
000693\end{array}$ & sox7 & -1.49 & $7.80 \mathrm{E}-17$ \\
\hline $\begin{array}{l}\text { ENSXETG00000 } \\
018919\end{array}$ & cnn3 & 1.44 & $5.10 \mathrm{E}-06$ & $\begin{array}{l}\text { ENSXETG00000 } \\
010195\end{array}$ & c9orf96 & -1.49 & 1.85E-03 \\
\hline $\begin{array}{l}\text { ENSXETG00000 } \\
026104\end{array}$ & st3gal6 & 1.44 & 1.62E-02 & $\begin{array}{l}\text { ENSXETG00000 } \\
001452\end{array}$ & $m d m 1$ & -1.49 & 7.51E-04 \\
\hline $\begin{array}{l}\text { ENSXETG00000 } \\
014751\end{array}$ & atp1b3 & 1.43 & $1.25 \mathrm{E}-03$ & $\begin{array}{l}\text { ENSXETG00000 } \\
032973\end{array}$ & iqca1 & -1.49 & 4.11E-06 \\
\hline $\begin{array}{l}\text { ENSXETG00000 } \\
012324\end{array}$ & $\operatorname{lin} 28 a$ & 1.42 & $4.50 \mathrm{E}-02$ & $\begin{array}{l}\text { ENSXETG00000 } \\
019904\end{array}$ & Irrc3b & -1.48 & 1.06E-02 \\
\hline $\begin{array}{l}\text { ENSXETG00000 } \\
025992\end{array}$ & cdh3 & 1.41 & $5.13 \mathrm{E}-12$ & $\begin{array}{l}\text { ENSXETG00000 } \\
033755\end{array}$ & speg & -1.48 & $2.90 \mathrm{E}-02$ \\
\hline $\begin{array}{l}\text { ENSXETG00000 } \\
000076\end{array}$ & mapkapk3 & 1.41 & $1.18 \mathrm{E}-02$ & $\begin{array}{l}\text { ENSXETG00000 } \\
016710\end{array}$ & & -1.48 & $7.58 \mathrm{E}-09$ \\
\hline $\begin{array}{l}\text { ENSXETG00000 } \\
011231\end{array}$ & $\mathrm{cmtm3}$ & 1.39 & 1.31E-02 & $\begin{array}{l}\text { ENSXETG00000 } \\
010185\end{array}$ & $\operatorname{dixdc1}$ & -1.48 & 1.33E-03 \\
\hline $\begin{array}{l}\text { ENSXETG00000 } \\
010393\end{array}$ & & 1.39 & $1.36 \mathrm{E}-02$ & $\begin{array}{l}\text { ENSXETG00000 } \\
032155\end{array}$ & & -1.47 & $8.54 \mathrm{E}-04$ \\
\hline $\begin{array}{l}\text { ENSXETG00000 } \\
003952\end{array}$ & nrarp & 1.39 & 5.89E-04 & $\begin{array}{l}\text { ENSXETG00000 } \\
000335\end{array}$ & $c d k / 2$ & -1.47 & $9.22 \mathrm{E}-06$ \\
\hline $\begin{array}{l}\text { ENSXETG00000 } \\
017085\end{array}$ & $p k p 1$ & 1.39 & $3.86 \mathrm{E}-02$ & $\begin{array}{l}\text { ENSXETG00000 } \\
013463\end{array}$ & zmynd12 & -1.47 & 3.37E-07 \\
\hline $\begin{array}{l}\text { ENSXETG00000 } \\
031404\end{array}$ & draxin & 1.38 & 1.57E-02 & $\begin{array}{l}\text { ENSXETG00000 } \\
010186\end{array}$ & coro2a & -1.47 & 4.42E-02 \\
\hline $\begin{array}{l}\text { ENSXETG00000 } \\
015391\end{array}$ & cadm1 & 1.37 & $6.52 \mathrm{E}-04$ & $\begin{array}{l}\text { ENSXETG00000 } \\
003349\end{array}$ & poc5 & -1.47 & $3.57 \mathrm{E}-06$ \\
\hline $\begin{array}{l}\text { ENSXETG00000 } \\
025060\end{array}$ & shprh & 1.37 & $3.85 \mathrm{E}-03$ & $\begin{array}{l}\text { ENSXETG00000 } \\
001383\end{array}$ & c1orf158 & -1.47 & $2.84 \mathrm{E}-08$ \\
\hline $\begin{array}{l}\text { ENSXETG00000 } \\
030338\end{array}$ & & 1.36 & $5.28 \mathrm{E}-03$ & $\begin{array}{l}\text { ENSXETG00000 } \\
021173\end{array}$ & ncoa1 & -1.46 & $8.28 \mathrm{E}-04$ \\
\hline
\end{tabular}




\begin{tabular}{|c|c|c|c|}
\hline $\begin{array}{l}\text { ENSXETG00000 } \\
021749\end{array}$ & & 1.36 & $4.26 \mathrm{E}-03$ \\
\hline $\begin{array}{l}\text { ENSXETG00000 } \\
020160\end{array}$ & pros1 & 1.35 & 3.74E-02 \\
\hline $\begin{array}{l}\text { ENSXETG00000 } \\
027751\end{array}$ & marcks & 1.35 & 1.54E-02 \\
\hline $\begin{array}{l}\text { ENSXETG00000 } \\
004371\end{array}$ & $\operatorname{tspan} 1$ & 1.34 & 1.06E-03 \\
\hline $\begin{array}{l}\text { ENSXETG00000 } \\
008603\end{array}$ & akt1 & 1.33 & 8.43E-04 \\
\hline $\begin{array}{l}\text { ENSXETG00000 } \\
020280\end{array}$ & fam171a1 & 1.33 & 7.09E-03 \\
\hline $\begin{array}{l}\text { ENSXETG00000 } \\
002238\end{array}$ & akap2 & 1.32 & $1.99 \mathrm{E}-04$ \\
\hline $\begin{array}{l}\text { ENSXETG00000 } \\
025083\end{array}$ & c6orf35 & 1.32 & $3.59 \mathrm{E}-02$ \\
\hline $\begin{array}{l}\text { ENSXETG00000 } \\
005091\end{array}$ & Irp4 & 1.31 & 2.47E-05 \\
\hline $\begin{array}{l}\text { ENSXETG00000 } \\
016127\end{array}$ & $c b l b$ & 1.31 & $1.82 \mathrm{E}-05$ \\
\hline $\begin{array}{l}\text { ENSXETG00000 } \\
033405\end{array}$ & cbr1 & 1.30 & 1.43E-13 \\
\hline $\begin{array}{l}\text { ENSXETG00000 } \\
025368\end{array}$ & best2 & 1.30 & 7.43E-03 \\
\hline $\begin{array}{l}\text { ENSXETG00000 } \\
018074\end{array}$ & rasgrp1 & 1.29 & 1.14E-02 \\
\hline $\begin{array}{l}\text { ENSXETG00000 } \\
016775\end{array}$ & fgfr1 & 1.29 & 2.64E-07 \\
\hline $\begin{array}{l}\text { ENSXETG00000 } \\
016111\end{array}$ & s/c27a2 & 1.29 & 3.30E-06 \\
\hline $\begin{array}{l}\text { ENSXETG00000 } \\
022029\end{array}$ & pfkfb1 & 1.29 & $1.10 \mathrm{E}-12$ \\
\hline $\begin{array}{l}\text { ENSXETG00000 } \\
018375\end{array}$ & anxa11 & 1.29 & 4.86E-04 \\
\hline $\begin{array}{l}\text { ENSXETG00000 } \\
005683\end{array}$ & & 1.28 & $6.74 \mathrm{E}-05$ \\
\hline $\begin{array}{l}\text { ENSXETG00000 } \\
011781\end{array}$ & rab11fip5 & 1.28 & $3.22 \mathrm{E}-02$ \\
\hline $\begin{array}{l}\text { ENSXETG00000 } \\
018359\end{array}$ & $z m i z 1$ & 1.27 & 4.54E-04 \\
\hline $\begin{array}{l}\text { ENSXETG00000 } \\
010302\end{array}$ & chga & 1.27 & $1.25 \mathrm{E}-02$ \\
\hline $\begin{array}{l}\text { ENSXETG00000 } \\
027667\end{array}$ & c9orf6 & 1.27 & $6.90 \mathrm{E}-08$ \\
\hline $\begin{array}{l}\text { ENSXETG00000 } \\
009866\end{array}$ & znf503 & 1.27 & 2.61E-05 \\
\hline $\begin{array}{l}\text { ENSXETG00000 } \\
033367\end{array}$ & tmem2 & 1.27 & $5.59 \mathrm{E}-07$ \\
\hline $\begin{array}{l}\text { ENSXETG00000 } \\
015500\end{array}$ & mllt3 & 1.26 & 3.26E-02 \\
\hline $\begin{array}{l}\text { ENSXETG00000 } \\
010460\end{array}$ & nos1ap & 1.26 & $1.23 \mathrm{E}-05$ \\
\hline $\begin{array}{l}\text { ENSXETG00000 } \\
019965\end{array}$ & kcnq1 & 1.25 & $1.52 \mathrm{E}-07$ \\
\hline $\begin{array}{l}\text { ENSXETG00000 } \\
019763\end{array}$ & mospd1 & 1.25 & 1.30E-04 \\
\hline $\begin{array}{l}\text { ENSXETG00000 } \\
011217\end{array}$ & thbs 1 & 1.24 & $2.50 \mathrm{E}-02$ \\
\hline $\begin{array}{l}\text { ENSXETG00000 } \\
011962\end{array}$ & dact1 & 1.24 & 1.10E-03 \\
\hline $\begin{array}{l}\text { ENSXETG00000 } \\
004387\end{array}$ & cept1 & 1.24 & $6.80 \mathrm{E}-04$ \\
\hline $\begin{array}{l}\text { ENSXETG00000 } \\
019267\end{array}$ & c6orf203 & 1.23 & $2.55 \mathrm{E}-02$ \\
\hline $\begin{array}{l}\text { ENSXETG00000 } \\
020924\end{array}$ & ippk & 1.23 & 3.24E-02 \\
\hline $\begin{array}{l}\text { ENSXETG00000 } \\
025190\end{array}$ & Irrc8b & 1.23 & $5.40 \mathrm{E}-03$ \\
\hline $\begin{array}{l}\text { ENSXETG00000 } \\
016563\end{array}$ & tcf12 & 1.23 & $6.41 \mathrm{E}-06$ \\
\hline $\begin{array}{l}\text { ENSXETG00000 } \\
010357\end{array}$ & nme3 & 1.22 & $2.58 \mathrm{E}-02$ \\
\hline $\begin{array}{l}\text { ENSXETG00000 } \\
017315\end{array}$ & adcy9 & 1.21 & $4.81 \mathrm{E}-07$ \\
\hline
\end{tabular}

\begin{tabular}{|c|c|c|c|}
\hline $\begin{array}{l}\text { ENSXETG00000 } \\
004761\end{array}$ & $\begin{array}{l}x b \text {-gene- } \\
5817486\end{array}$ & -1.46 & $3.65 \mathrm{E}-03$ \\
\hline $\begin{array}{l}\text { ENSXETG00000 } \\
017409\end{array}$ & kiaa0513 & -1.46 & $9.09 \mathrm{E}-04$ \\
\hline $\begin{array}{l}\text { ENSXETG00000 } \\
006299\end{array}$ & xepsin & -1.46 & 1.50E-02 \\
\hline $\begin{array}{l}\text { ENSXETG00000 } \\
021376\end{array}$ & mpped2 & -1.46 & $3.99 \mathrm{E}-02$ \\
\hline $\begin{array}{l}\text { ENSXETG00000 } \\
019539\end{array}$ & wdr16 & -1.46 & 3.67E-14 \\
\hline $\begin{array}{l}\text { ENSXETG00000 } \\
006312\end{array}$ & casz1 & -1.46 & $1.69 \mathrm{E}-11$ \\
\hline $\begin{array}{l}\text { ENSXETG00000 } \\
000118\end{array}$ & slc2a12 & -1.46 & 4.79E-02 \\
\hline $\begin{array}{l}\text { ENSXETG00000 } \\
013421\end{array}$ & crocc & -1.46 & $5.04 \mathrm{E}-04$ \\
\hline $\begin{array}{l}\text { ENSXETG00000 } \\
030034\end{array}$ & Irrc73 & -1.45 & $1.81 \mathrm{E}-02$ \\
\hline $\begin{array}{l}\text { ENSXETG00000 } \\
025205\end{array}$ & $\begin{array}{l}\text { xb-gene- } \\
5922677\end{array}$ & -1.45 & $3.09 \mathrm{E}-08$ \\
\hline $\begin{array}{l}\text { ENSXETG00000 } \\
012154\end{array}$ & & -1.45 & 7.82E-04 \\
\hline $\begin{array}{l}\text { ENSXETG00000 } \\
013950\end{array}$ & $a k 7$ & -1.45 & 5.91E-09 \\
\hline $\begin{array}{l}\text { ENSXETG00000 } \\
016747\end{array}$ & $\mathrm{rg} / 3$ & -1.45 & $3.15 \mathrm{E}-03$ \\
\hline $\begin{array}{l}\text { ENSXETG00000 } \\
018928\end{array}$ & c2orf77 & -1.45 & 9.09E-04 \\
\hline $\begin{array}{l}\text { ENSXETG00000 } \\
023781\end{array}$ & c2orf50 & -1.45 & 1.33E-04 \\
\hline $\begin{array}{l}\text { ENSXETG00000 } \\
005240\end{array}$ & $d c d c 2 b$ & -1.45 & $6.88 \mathrm{E}-05$ \\
\hline $\begin{array}{l}\text { ENSXETG00000 } \\
008873\end{array}$ & kndc1 & -1.44 & $2.63 \mathrm{E}-03$ \\
\hline $\begin{array}{l}\text { ENSXETG00000 } \\
021705\end{array}$ & & -1.44 & $7.51 \mathrm{E}-04$ \\
\hline $\begin{array}{l}\text { ENSXETG00000 } \\
013234\end{array}$ & $c d c 14 a$ & -1.44 & $8.29 \mathrm{E}-07$ \\
\hline $\begin{array}{l}\text { ENSXETG00000 } \\
022874\end{array}$ & $w d r 49$ & -1.44 & 1.53E-04 \\
\hline $\begin{array}{l}\text { ENSXETG00000 } \\
017416\end{array}$ & fam92b & -1.44 & $2.54 \mathrm{E}-05$ \\
\hline $\begin{array}{l}\text { ENSXETG00000 } \\
001591\end{array}$ & cetn4 & -1.43 & 1.89E-05 \\
\hline $\begin{array}{l}\text { ENSXETG00000 } \\
032161\end{array}$ & morn3 & -1.43 & $9.27 \mathrm{E}-07$ \\
\hline $\begin{array}{l}\text { ENSXETG00000 } \\
025413\end{array}$ & mest & -1.43 & 2.34E-02 \\
\hline $\begin{array}{l}\text { ENSXETG00000 } \\
011276\end{array}$ & slc22a31 & -1.43 & $5.65 \mathrm{E}-04$ \\
\hline $\begin{array}{l}\text { ENSXETG00000 } \\
014603\end{array}$ & slc7a8 & -1.43 & $3.49 \mathrm{E}-05$ \\
\hline $\begin{array}{l}\text { ENSXETG00000 } \\
005961\end{array}$ & & -1.43 & 4.49E-04 \\
\hline $\begin{array}{l}\text { ENSXETG00000 } \\
000061\end{array}$ & dnal1 & -1.43 & $1.42 \mathrm{E}-08$ \\
\hline $\begin{array}{l}\text { ENSXETG00000 } \\
010363\end{array}$ & tekt1 & -1.43 & 1.15E-09 \\
\hline $\begin{array}{l}\text { ENSXETG00000 } \\
020932\end{array}$ & esrrg & -1.42 & 7.37E-03 \\
\hline $\begin{array}{l}\text { ENSXETG00000 } \\
032986\end{array}$ & & -1.42 & 7.63E-08 \\
\hline $\begin{array}{l}\text { ENSXETG00000 } \\
026362\end{array}$ & $\begin{array}{l}x b-g e n e- \\
5902318\end{array}$ & -1.42 & 2.88E-03 \\
\hline $\begin{array}{l}\text { ENSXETG00000 } \\
013823\end{array}$ & elmo3 & -1.41 & 2.18E-03 \\
\hline $\begin{array}{l}\text { ENSXETG00000 } \\
012922\end{array}$ & pde $4 c$ & -1.41 & $2.28 \mathrm{E}-02$ \\
\hline $\begin{array}{l}\text { ENSXETG00000 } \\
004839\end{array}$ & maob & -1.41 & $1.58 \mathrm{E}-02$ \\
\hline $\begin{array}{l}\text { ENSXETG00000 } \\
018269\end{array}$ & сер89 & -1.41 & 1.06E-05 \\
\hline $\begin{array}{l}\text { ENSXETG00000 } \\
012560\end{array}$ & $c 2 c d 3$ & -1.41 & $4.28 \mathrm{E}-06$ \\
\hline
\end{tabular}




\begin{tabular}{|c|c|c|c|c|c|c|c|}
\hline $\begin{array}{l}\text { ENSXETG00000 } \\
007197\end{array}$ & dnajc4 & 1.21 & 4.27E-03 & \begin{tabular}{|l|} 
ENSXETG00000 \\
002998
\end{tabular} & c1orf173 & -1.41 & 7.13E-03 \\
\hline $\begin{array}{l}\text { ENSXETG00000 } \\
017793\end{array}$ & $z c 4 h 2$ & 1.21 & $4.92 \mathrm{E}-03$ & $\begin{array}{l}\text { ENSXETG00000 } \\
024765\end{array}$ & dynlrb2 & -1.41 & 1.97E-05 \\
\hline $\begin{array}{l}\text { ENSXETG00000 } \\
030893\end{array}$ & & 1.21 & 4.43E-02 & $\begin{array}{l}\text { ENSXETG00000 } \\
034073\end{array}$ & $m d h 1 b$ & -1.41 & 4.57E-06 \\
\hline $\begin{array}{l}\text { ENSXETG00000 } \\
026157\end{array}$ & cdk5rap2 & 1.21 & $3.04 \mathrm{E}-02$ & $\begin{array}{l}\text { ENSXETG00000 } \\
016262\end{array}$ & enpp4 & -1.41 & 3.35E-02 \\
\hline $\begin{array}{l}\text { ENSXETG00000 } \\
033372\end{array}$ & & 1.20 & $2.81 \mathrm{E}-02$ & $\begin{array}{l}\text { ENSXETG00000 } \\
011397\end{array}$ & neurl & -1.40 & $9.95 \mathrm{E}-05$ \\
\hline $\begin{array}{l}\text { ENSXETG00000 } \\
027014\end{array}$ & traf4 & 1.20 & $4.72 \mathrm{E}-02$ & $\begin{array}{l}\text { ENSXETG00000 } \\
003974\end{array}$ & clic3 & -1.40 & $9.96 \mathrm{E}-05$ \\
\hline $\begin{array}{l}\text { ENSXETG00000 } \\
034081\end{array}$ & cul5 & 1.19 & $1.18 \mathrm{E}-02$ & $\begin{array}{l}\text { ENSXETG00000 } \\
022138\end{array}$ & iqub & -1.40 & 1.40E-08 \\
\hline $\begin{array}{l}\text { ENSXETG00000 } \\
017566\end{array}$ & smarca5 & 1.19 & $4.24 \mathrm{E}-08$ & $\begin{array}{l}\text { ENSXETG00000 } \\
006354\end{array}$ & kiaa1370 & -1.39 & $8.74 \mathrm{E}-08$ \\
\hline $\begin{array}{l}\text { ENSXETG00000 } \\
011565\end{array}$ & mdfic & 1.18 & $3.45 \mathrm{E}-02$ & $\begin{array}{l}\text { ENSXETG00000 } \\
013608 \\
\end{array}$ & cep112 & -1.39 & 1.33E-02 \\
\hline $\begin{array}{l}\text { ENSXETG00000 } \\
018620\end{array}$ & prrg1 & 1.18 & $1.50 \mathrm{E}-10$ & $\begin{array}{l}\text { ENSXETG00000 } \\
008631\end{array}$ & Ipar3 & -1.39 & $4.69 \mathrm{E}-03$ \\
\hline $\begin{array}{l}\text { ENSXETG00000 } \\
009218\end{array}$ & mynn & 1.18 & $6.04 \mathrm{E}-04$ & $\begin{array}{l}\text { ENSXETG00000 } \\
017777\end{array}$ & wdr52 & -1.39 & 7.33E-03 \\
\hline $\begin{array}{l}\text { ENSXETG00000 } \\
025335\end{array}$ & rnd3 & 1.17 & 1.47E-02 & \begin{tabular}{|l|} 
ENSXETG00000 \\
018653 \\
\end{tabular} & & -1.39 & 4.47E-02 \\
\hline $\begin{array}{l}\text { ENSXETG00000 } \\
014857\end{array}$ & ostm1 & 1.17 & $1.99 \mathrm{E}-06$ & $\begin{array}{l}\text { ENSXETG00000 } \\
019906\end{array}$ & nek10 & -1.39 & $3.52 \mathrm{E}-03$ \\
\hline $\begin{array}{l}\text { ENSXETG00000 } \\
011833\end{array}$ & ptprd & 1.17 & 1.36E-03 & $\begin{array}{l}\text { ENSXETG00000 } \\
030298\end{array}$ & pifo & -1.39 & 4.22E-02 \\
\hline $\begin{array}{l}\text { ENSXETG00000 } \\
012186\end{array}$ & ccnj & 1.16 & $1.41 \mathrm{E}-03$ & $\begin{array}{l}\text { ENSXETG00000 } \\
014097\end{array}$ & rarres1 & -1.38 & $1.00 \mathrm{E}-03$ \\
\hline $\begin{array}{l}\text { ENSXETG00000 } \\
011897\end{array}$ & eno1 & 1.15 & $6.82 \mathrm{E}-03$ & $\begin{array}{l}\text { ENSXETG00000 } \\
022093\end{array}$ & dnah7 & -1.38 & 7.46E-05 \\
\hline $\begin{array}{l}\text { ENSXETG00000 } \\
012289\end{array}$ & rps6ka1 & 1.15 & 1.65E-04 & $\begin{array}{l}\text { ENSXETG00000 } \\
023071\end{array}$ & fam102a & -1.38 & 3.07E-02 \\
\hline $\begin{array}{l}\text { ENSXETG00000 } \\
023793\end{array}$ & greb1 & 1.15 & $4.28 \mathrm{E}-03$ & $\begin{array}{l}\text { ENSXETG00000 } \\
005871\end{array}$ & fam149a & -1.37 & 1.57E-02 \\
\hline $\begin{array}{l}\text { ENSXETG00000 } \\
013793\end{array}$ & setd 6 & 1.15 & $5.09 \mathrm{E}-06$ & $\begin{array}{l}\text { ENSXETG00000 } \\
032269\end{array}$ & Irrfip1 & -1.37 & $6.92 \mathrm{E}-04$ \\
\hline $\begin{array}{l}\text { ENSXETG00000 } \\
010313\end{array}$ & otub2 & 1.14 & $2.75 \mathrm{E}-02$ & $\begin{array}{l}\text { ENSXETG00000 } \\
026283\end{array}$ & gjb1 & -1.37 & 1.16E-02 \\
\hline $\begin{array}{l}\text { ENSXETG00000 } \\
030447\end{array}$ & dusp8 & 1.14 & $3.97 \mathrm{E}-03$ & $\begin{array}{l}\text { ENSXETG00000 } \\
022580\end{array}$ & $n h s / 2$ & -1.37 & $2.94 \mathrm{E}-03$ \\
\hline $\begin{array}{l}\text { ENSXETG00000 } \\
012743\end{array}$ & rcor1 & 1.13 & $5.45 \mathrm{E}-03$ & $\begin{array}{l}\text { ENSXETG00000 } \\
007409\end{array}$ & rapgef5 & -1.36 & 8.92E-07 \\
\hline $\begin{array}{l}\text { ENSXETG00000 } \\
021414\end{array}$ & egln1 & 1.12 & $1.22 \mathrm{E}-02$ & $\begin{array}{l}\text { ENSXETG00000 } \\
009576\end{array}$ & oat.2 & -1.36 & $5.05 \mathrm{E}-09$ \\
\hline $\begin{array}{l}\text { ENSXETG00000 } \\
010841\end{array}$ & dock7 & 1.12 & $6.22 \mathrm{E}-06$ & $\begin{array}{l}\text { ENSXETG00000 } \\
004679\end{array}$ & $10 x$ & -1.36 & 3.17E-02 \\
\hline $\begin{array}{l}\text { ENSXETG00000 } \\
005431\end{array}$ & sema3d & 1.12 & $4.72 \mathrm{E}-02$ & $\begin{array}{l}\text { ENSXETG00000 } \\
032814\end{array}$ & clhc1 & -1.36 & 3.35E-06 \\
\hline $\begin{array}{l}\text { ENSXETG00000 } \\
024679\end{array}$ & spats2 & 1.12 & $6.57 \mathrm{E}-06$ & $\begin{array}{l}\text { ENSXETG00000 } \\
023923\end{array}$ & Irrc23 & -1.36 & 8.85E-09 \\
\hline $\begin{array}{l}\text { ENSXETG00000 } \\
004155\end{array}$ & abi2 & 1.11 & 7.67E-07 & $\begin{array}{l}\text { ENSXETG00000 } \\
025131\end{array}$ & sgpp1 & -1.35 & $1.25 \mathrm{E}-04$ \\
\hline $\begin{array}{l}\text { ENSXETG00000 } \\
024397\end{array}$ & nr1d1 & 1.10 & $1.28 \mathrm{E}-02$ & $\begin{array}{l}\text { ENSXETG00000 } \\
033099\end{array}$ & jag2 & -1.35 & $2.94 \mathrm{E}-03$ \\
\hline $\begin{array}{l}\text { ENSXETG00000 } \\
003253\end{array}$ & pik3r3 & 1.10 & $8.28 \mathrm{E}-03$ & $\begin{array}{l}\text { ENSXETG00000 } \\
031301\end{array}$ & $c d c 20 b$ & -1.35 & $9.59 \mathrm{E}-05$ \\
\hline $\begin{array}{l}\text { ENSXETG00000 } \\
005342\end{array}$ & arhgap6 & 1.10 & $1.28 \mathrm{E}-03$ & \begin{tabular}{|l|} 
ENSXETG00000 \\
013238 \\
\end{tabular} & fos & -1.35 & 4.00E-03 \\
\hline $\begin{array}{l}\text { ENSXETG00000 } \\
011818\end{array}$ & ryk & 1.08 & 7.21E-04 & $\begin{array}{l}\text { ENSXETG00000 } \\
015673 \\
\end{array}$ & arl11 & -1.35 & 1.33E-02 \\
\hline $\begin{array}{l}\text { ENSXETG00000 } \\
017437\end{array}$ & cdk2ap2 & 1.08 & 2.16E-02 & \begin{tabular}{|l|} 
ENSXETG00000 \\
026793
\end{tabular} & tmem30b & -1.35 & $1.44 \mathrm{E}-03$ \\
\hline $\begin{array}{l}\text { ENSXETG00000 } \\
012160\end{array}$ & srebf1 & 1.08 & 2.11E-04 & $\begin{array}{l}\text { ENSXETG00000 } \\
002371\end{array}$ & kif27 & -1.35 & 2.63E-03 \\
\hline $\begin{array}{l}\text { ENSXETG00000 } \\
030743\end{array}$ & $m b$ & 1.08 & $2.10 \mathrm{E}-04$ & $\begin{array}{l}\text { ENSXETG00000 } \\
021433\end{array}$ & snta1 & -1.35 & 1.15E-04 \\
\hline $\begin{array}{l}\text { ENSXETG00000 } \\
000543\end{array}$ & gldc & 1.08 & 4.85E-02 & $\begin{array}{l}\text { ENSXETG00000 } \\
032805\end{array}$ & & -1.34 & 4.75E-02 \\
\hline $\begin{array}{l}\text { ENSXETG00000 } \\
011111\end{array}$ & hes3.1 & 1.08 & $5.91 \mathrm{E}-03$ & $\begin{array}{l}\text { ENSXETG00000 } \\
002233 \\
\end{array}$ & fam161a & -1.34 & 2.10E-04 \\
\hline $\begin{array}{l}\text { ENSXETG00000 } \\
001573\end{array}$ & spry1 & 1.07 & 3.26E-02 & $\begin{array}{l}\text { ENSXETG00000 } \\
010212\end{array}$ & hopx & -1.34 & $3.49 \mathrm{E}-03$ \\
\hline
\end{tabular}




\begin{tabular}{|c|c|c|c|}
\hline $\begin{array}{l}\text { ENSXETG00000 } \\
014939\end{array}$ & smo & 1.06 & 4.08E-02 \\
\hline $\begin{array}{l}\text { ENSXETG00000 } \\
012949\end{array}$ & ctps & 1.06 & $3.88 \mathrm{E}-06$ \\
\hline $\begin{array}{l}\text { ENSXETG00000 } \\
015984\end{array}$ & nol12 & 1.06 & $2.84 \mathrm{E}-02$ \\
\hline $\begin{array}{l}\text { ENSXETG00000 } \\
011847\end{array}$ & med20 & 1.05 & 4.16E-04 \\
\hline $\begin{array}{l}\text { ENSXETG00000 } \\
007756\end{array}$ & shf & 1.05 & $2.88 \mathrm{E}-02$ \\
\hline $\begin{array}{l}\text { ENSXETG00000 } \\
012714\end{array}$ & arhgef37 & 1.05 & 3.32E-05 \\
\hline $\begin{array}{l}\text { ENSXETG00000 } \\
007514\end{array}$ & ipo8 & 1.05 & $1.27 \mathrm{E}-02$ \\
\hline $\begin{array}{l}\text { ENSXETG00000 } \\
009119\end{array}$ & Irr1 & 1.04 & 3.06E-02 \\
\hline $\begin{array}{l}\text { ENSXETG00000 } \\
013749\end{array}$ & polr1b & 1.04 & 1.37E-06 \\
\hline $\begin{array}{l}\text { ENSXETG00000 } \\
021731\end{array}$ & epha2 & 1.03 & 3.73E-04 \\
\hline $\begin{array}{l}\text { ENSXETG00000 } \\
014976\end{array}$ & slc39a6 & 1.03 & $2.94 \mathrm{E}-02$ \\
\hline $\begin{array}{l}\text { ENSXETG00000 } \\
017414\end{array}$ & ubiad1 & 1.03 & $2.65 \mathrm{E}-03$ \\
\hline $\begin{array}{l}\text { ENSXETG00000 } \\
003247\end{array}$ & znf451 & 1.03 & $2.14 \mathrm{E}-03$ \\
\hline $\begin{array}{l}\text { ENSXETG00000 } \\
022626\end{array}$ & prmt5 & 1.02 & $9.08 \mathrm{E}-04$ \\
\hline $\begin{array}{l}\text { ENSXETG00000 } \\
021932\end{array}$ & c18orf21 & 1.02 & $3.88 \mathrm{E}-04$ \\
\hline $\begin{array}{l}\text { ENSXETG00000 } \\
015231\end{array}$ & sulf1 & 1.01 & $3.12 \mathrm{E}-02$ \\
\hline $\begin{array}{l}\text { ENSXETG00000 } \\
021691\end{array}$ & mapk8 & 1.01 & $2.22 \mathrm{E}-03$ \\
\hline $\begin{array}{l}\text { ENSXETG00000 } \\
012163\end{array}$ & ppp1r7 & 1.01 & $1.87 \mathrm{E}-03$ \\
\hline $\begin{array}{l}\text { ENSXETG00000 } \\
021431\end{array}$ & cbfa2t2 & 1.01 & 1.85E-05 \\
\hline $\begin{array}{l}\text { ENSXETG00000 } \\
020027\end{array}$ & $c b f b$ & 1.01 & $1.74 \mathrm{E}-02$ \\
\hline $\begin{array}{l}\text { ENSXETG00000 } \\
012969\end{array}$ & $r c c 1$ & 1.01 & $2.38 \mathrm{E}-03$ \\
\hline $\begin{array}{l}\text { ENSXETG00000 } \\
018603\end{array}$ & $m v k$ & 1.00 & 8.54E-05 \\
\hline $\begin{array}{l}\text { ENSXETG00000 } \\
015037\end{array}$ & taf1a & 1.00 & 1.76E-02 \\
\hline
\end{tabular}

\begin{tabular}{|c|c|c|c|}
\hline $\begin{array}{l}\text { ENSXETG00000 } \\
021129\end{array}$ & bmper & -1.34 & $8.22 \mathrm{E}-03$ \\
\hline $\begin{array}{l}\text { ENSXETG00000 } \\
018451\end{array}$ & dlec1 & -1.34 & 4.35E-03 \\
\hline $\begin{array}{l}\text { ENSXETG00000 } \\
031212\end{array}$ & Irrc61 & -1.34 & 1.09E-03 \\
\hline $\begin{array}{l}\text { ENSXETG00000 } \\
007364\end{array}$ & slc4a1 & -1.33 & 4.86E-04 \\
\hline $\begin{array}{l}\text { ENSXETG00000 } \\
000511\end{array}$ & tubal3 & -1.33 & 1.37E-06 \\
\hline $\begin{array}{l}\text { ENSXETG00000 } \\
025129\end{array}$ & fam154b & -1.32 & 4.36E-07 \\
\hline $\begin{array}{l}\text { ENSXETG00000 } \\
019254\end{array}$ & & -1.32 & 2.59E-09 \\
\hline $\begin{array}{l}\text { ENSXETG00000 } \\
011664\end{array}$ & $t t c 12$ & -1.32 & 1.31E-03 \\
\hline $\begin{array}{l}\text { ENSXETG00000 } \\
013794\end{array}$ & c20orf12 & -1.32 & 3.25E-07 \\
\hline $\begin{array}{l}\text { ENSXETG00000 } \\
006039\end{array}$ & Irrc56 & -1.32 & 1.06E-02 \\
\hline $\begin{array}{l}\text { ENSXETG00000 } \\
005411\end{array}$ & meig1 & -1.32 & $9.85 \mathrm{E}-05$ \\
\hline $\begin{array}{l}\text { ENSXETG00000 } \\
032730\end{array}$ & & -1.31 & 7.02E-04 \\
\hline $\begin{array}{l}\text { ENSXETG00000 } \\
021807\end{array}$ & cdkl5 & -1.31 & 3.26E-02 \\
\hline $\begin{array}{l}\text { ENSXETG00000 } \\
020259\end{array}$ & fam195a & -1.31 & $1.98 \mathrm{E}-04$ \\
\hline $\begin{array}{l}\text { ENSXETG00000 } \\
024054\end{array}$ & rsph10b & -1.31 & $8.25 \mathrm{E}-05$ \\
\hline $\begin{array}{l}\text { ENSXETG00000 } \\
004553\end{array}$ & armc4 & -1.30 & 3.19E-06 \\
\hline $\begin{array}{l}\text { ENSXETG00000 } \\
008083\end{array}$ & sptlc3 & -1.29 & 3.39E-06 \\
\hline $\begin{array}{l}\text { ENSXETG00000 } \\
019807\end{array}$ & cntrl & -1.29 & 1.05E-06 \\
\hline $\begin{array}{l}\text { ENSXETG00000 } \\
000221\end{array}$ & sertad4 & -1.29 & 2.78E-02 \\
\hline $\begin{array}{l}\text { ENSXETG00000 } \\
001621\end{array}$ & fam83b & -1.29 & 3.56E-03 \\
\hline $\begin{array}{l}\text { ENSXETG00000 } \\
019246\end{array}$ & & -1.29 & 4.71E-04 \\
\hline $\begin{array}{l}\text { ENSXETG00000 } \\
021922\end{array}$ & $p f k p$ & -1.29 & 4.43E-02 \\
\hline $\begin{array}{l}\text { ENSXETG00000 } \\
010513\end{array}$ & rhobtb2 & -1.29 & $2.63 \mathrm{E}-02$ \\
\hline $\begin{array}{l}\text { ENSXETG00000 } \\
030628\end{array}$ & cep41 & -1.29 & $9.00 \mathrm{E}-10$ \\
\hline $\begin{array}{l}\text { ENSXETG00000 } \\
003474\end{array}$ & eya2 & -1.29 & 3.26E-02 \\
\hline $\begin{array}{l}\text { ENSXETG00000 } \\
008832\end{array}$ & syt7 & -1.29 & 1.15E-02 \\
\hline $\begin{array}{l}\text { ENSXETG00000 } \\
000853\end{array}$ & nfatc1 & -1.28 & $1.68 \mathrm{E}-04$ \\
\hline $\begin{array}{l}\text { ENSXETG00000 } \\
020342\end{array}$ & Irrc49 & -1.28 & 6.07E-05 \\
\hline $\begin{array}{l}\text { ENSXETG00000 } \\
018749\end{array}$ & $b 9 d 2$ & -1.28 & 2.85E-05 \\
\hline $\begin{array}{l}\text { ENSXETG00000 } \\
025359\end{array}$ & spef2 & -1.28 & 5.05E-09 \\
\hline $\begin{array}{l}\text { ENSXETG00000 } \\
007441\end{array}$ & c7orf63 & -1.28 & 5.83E-06 \\
\hline $\begin{array}{l}\text { ENSXETG00000 } \\
023547\end{array}$ & cidec & -1.28 & $1.50 \mathrm{E}-06$ \\
\hline $\begin{array}{l}\text { ENSXETG00000 } \\
018054\end{array}$ & c3orf15 & -1.28 & 1.46E-06 \\
\hline $\begin{array}{l}\text { ENSXETG00000 } \\
021532\end{array}$ & atp6v1b1 & -1.28 & $2.40 \mathrm{E}-02$ \\
\hline $\begin{array}{l}\text { ENSXETG00000 } \\
010337\end{array}$ & ern2 & -1.28 & $8.25 \mathrm{E}-05$ \\
\hline $\begin{array}{l}\text { ENSXETG00000 } \\
025736\end{array}$ & $i d 4$ & -1.27 & 4.86E-04 \\
\hline $\begin{array}{l}\text { ENSXETG00000 } \\
017121\end{array}$ & $\operatorname{ccdc} 67$ & -1.27 & $2.00 \mathrm{E}-07$ \\
\hline
\end{tabular}




\begin{tabular}{|c|c|c|c|}
\hline $\begin{array}{l}\text { ENSXETG00000 } \\
030250\end{array}$ & & -1.27 & $1.39 \mathrm{E}-08$ \\
\hline $\begin{array}{l}\text { ENSXETG00000 } \\
005339\end{array}$ & ankrd9 & -1.27 & $2.69 \mathrm{E}-05$ \\
\hline $\begin{array}{l}\text { ENSXETG00000 } \\
017407\end{array}$ & $f b \times 031$ & -1.27 & 3.35E-06 \\
\hline $\begin{array}{l}\text { ENSXETG00000 } \\
021693\end{array}$ & c12orf63 & -1.27 & 1.12E-02 \\
\hline $\begin{array}{l}\text { ENSXETG00000 } \\
017719\end{array}$ & $p d e 7 b$ & -1.27 & $1.47 \mathrm{E}-03$ \\
\hline $\begin{array}{l}\text { ENSXETG00000 } \\
017468\end{array}$ & & -1.27 & 3.35E-02 \\
\hline $\begin{array}{l}\text { ENSXETG00000 } \\
015230\end{array}$ & c8orf34 & -1.26 & 4.70E-02 \\
\hline $\begin{array}{l}\text { ENSXETG00000 } \\
008245\end{array}$ & $\operatorname{ctg} f$ & -1.26 & $1.35 \mathrm{E}-02$ \\
\hline $\begin{array}{l}\text { ENSXETG00000 } \\
025254\end{array}$ & ccdc60 & -1.26 & 3.53E-02 \\
\hline $\begin{array}{l}\text { ENSXETG00000 } \\
000681\end{array}$ & mtus1 & -1.26 & 5.58E-08 \\
\hline $\begin{array}{l}\text { ENSXETG00000 } \\
016064\end{array}$ & tekt4 & -1.26 & $2.37 \mathrm{E}-07$ \\
\hline $\begin{array}{l}\text { ENSXETG00000 } \\
019283\end{array}$ & ptprn & -1.26 & $3.54 \mathrm{E}-02$ \\
\hline $\begin{array}{l}\text { ENSXETG00000 } \\
017281\end{array}$ & ttc16 & -1.26 & $7.21 \mathrm{E}-03$ \\
\hline $\begin{array}{l}\text { ENSXETG00000 } \\
030994\end{array}$ & kiaa0226 & -1.26 & 7.60E-03 \\
\hline $\begin{array}{l}\text { ENSXETG00000 } \\
033370\end{array}$ & & -1.25 & $1.04 \mathrm{E}-02$ \\
\hline $\begin{array}{l}\text { ENSXETG00000 } \\
003003\end{array}$ & Irrc45 & -1.25 & $2.07 \mathrm{E}-02$ \\
\hline $\begin{array}{l}\text { ENSXETG00000 } \\
014869\end{array}$ & efna5 & -1.25 & 1.06E-02 \\
\hline $\begin{array}{l}\text { ENSXETG00000 } \\
004470\end{array}$ & c9orf117 & -1.25 & 5.77E-05 \\
\hline $\begin{array}{l}\text { ENSXETG00000 } \\
024488\end{array}$ & c1orf177 & -1.25 & 1.97E-05 \\
\hline $\begin{array}{l}\text { ENSXETG00000 } \\
027042\end{array}$ & eif2c3 & -1.25 & $3.15 \mathrm{E}-03$ \\
\hline $\begin{array}{l}\text { ENSXETG00000 } \\
015826\end{array}$ & egfr & -1.25 & $5.39 \mathrm{E}-03$ \\
\hline $\begin{array}{l}\text { ENSXETG00000 } \\
016661\end{array}$ & & -1.25 & $2.20 \mathrm{E}-02$ \\
\hline $\begin{array}{l}\text { ENSXETG00000 } \\
007211\end{array}$ & esrra & -1.25 & $2.98 \mathrm{E}-05$ \\
\hline $\begin{array}{l}\text { ENSXETG00000 } \\
014773\end{array}$ & & -1.25 & $9.49 \mathrm{E}-03$ \\
\hline $\begin{array}{l}\text { ENSXETG00000 } \\
005016\end{array}$ & wrap73 & -1.25 & $3.28 \mathrm{E}-03$ \\
\hline $\begin{array}{l}\text { ENSXETG00000 } \\
031073\end{array}$ & katnal1 & -1.24 & 7.19E-03 \\
\hline $\begin{array}{l}\text { ENSXETG00000 } \\
030978\end{array}$ & cfap57 & -1.24 & $3.23 \mathrm{E}-06$ \\
\hline $\begin{array}{l}\text { ENSXETG00000 } \\
023111\end{array}$ & slc26a5 & -1.24 & 4.75E-02 \\
\hline $\begin{array}{l}\text { ENSXETG00000 } \\
001379\end{array}$ & pdgfa & -1.24 & 2.53E-02 \\
\hline $\begin{array}{l}\text { ENSXETG00000 } \\
026042\end{array}$ & rgs12 & -1.24 & $3.14 \mathrm{E}-02$ \\
\hline $\begin{array}{l}\text { ENSXETG00000 } \\
029983\end{array}$ & & -1.24 & $1.50 \mathrm{E}-02$ \\
\hline $\begin{array}{l}\text { ENSXETG00000 } \\
021994\end{array}$ & pip5kl1 & -1.24 & 3.01E-04 \\
\hline $\begin{array}{l}\text { ENSXETG00000 } \\
009776\end{array}$ & ezr & -1.24 & $2.16 \mathrm{E}-02$ \\
\hline $\begin{array}{l}\text { ENSXETG00000 } \\
000346\end{array}$ & $r b / 2$ & -1.23 & $3.82 \mathrm{E}-03$ \\
\hline $\begin{array}{l}\text { ENSXETG00000 } \\
000961\end{array}$ & ubr1 & -1.23 & $9.39 \mathrm{E}-03$ \\
\hline $\begin{array}{l}\text { ENSXETG00000 } \\
033951\end{array}$ & ccdc96 & -1.23 & $1.64 \mathrm{E}-03$ \\
\hline $\begin{array}{l}\text { ENSXETG00000 } \\
007177\end{array}$ & iqsec2 & -1.23 & 1.13E-02 \\
\hline
\end{tabular}




\begin{tabular}{|c|c|c|c|}
\hline $\begin{array}{l}\text { ENSXETG00000 } \\
010016\end{array}$ & pde9a & -1.23 & $5.57 \mathrm{E}-03$ \\
\hline $\begin{array}{l}\text { ENSXETG00000 } \\
006790\end{array}$ & tctex $1 d 1$ & -1.23 & $2.60 \mathrm{E}-09$ \\
\hline $\begin{array}{l}\text { ENSXETG00000 } \\
009243\end{array}$ & pvrl1 & -1.23 & $2.09 \mathrm{E}-13$ \\
\hline $\begin{array}{l}\text { ENSXETG00000 } \\
001752\end{array}$ & tuba8 & -1.23 & $1.48 \mathrm{E}-03$ \\
\hline $\begin{array}{l}\text { ENSXETG00000 } \\
033873\end{array}$ & & -1.23 & 4.29E-05 \\
\hline $\begin{array}{l}\text { ENSXETG00000 } \\
004522\end{array}$ & lypd6 & -1.23 & $1.71 \mathrm{E}-02$ \\
\hline $\begin{array}{l}\text { ENSXETG00000 } \\
018935\end{array}$ & bbs5 & -1.22 & 4.73E-07 \\
\hline $\begin{array}{l}\text { ENSXETG00000 } \\
007584\end{array}$ & $b a z 2 b$ & -1.22 & $5.37 \mathrm{E}-03$ \\
\hline $\begin{array}{l}\text { ENSXETG00000 } \\
013287\end{array}$ & atp2c2 & -1.22 & $2.24 \mathrm{E}-03$ \\
\hline $\begin{array}{l}\text { ENSXETG00000 } \\
010879\end{array}$ & rsph9 & -1.22 & $2.28 \mathrm{E}-03$ \\
\hline $\begin{array}{l}\text { ENSXETG00000 } \\
021049\end{array}$ & kit & -1.22 & $4.85 \mathrm{E}-04$ \\
\hline $\begin{array}{l}\text { ENSXETG00000 } \\
004424\end{array}$ & scube2 & -1.22 & 1.93E-02 \\
\hline $\begin{array}{l}\text { ENSXETG00000 } \\
016981\end{array}$ & Irrc9 & -1.22 & $1.60 \mathrm{E}-03$ \\
\hline $\begin{array}{l}\text { ENSXETG00000 } \\
004770\end{array}$ & fbxw9 & -1.22 & $2.01 \mathrm{E}-04$ \\
\hline $\begin{array}{l}\text { ENSXETG00000 } \\
030456\end{array}$ & mgst2 & -1.21 & $3.00 \mathrm{E}-03$ \\
\hline $\begin{array}{l}\text { ENSXETG00000 } \\
030879\end{array}$ & efcab11 & -1.21 & 1.13E-02 \\
\hline $\begin{array}{l}\text { ENSXETG00000 } \\
019577\end{array}$ & tekt3 & -1.21 & 1.27E-05 \\
\hline $\begin{array}{l}\text { ENSXETG00000 } \\
025737\end{array}$ & c4orf47 & -1.21 & $2.18 \mathrm{E}-06$ \\
\hline $\begin{array}{l}\text { ENSXETG00000 } \\
012662\end{array}$ & ccdc14 & -1.21 & 2.97E-02 \\
\hline $\begin{array}{l}\text { ENSXETG00000 } \\
006697\end{array}$ & egr1 & -1.20 & 3.32E-02 \\
\hline $\begin{array}{l}\text { ENSXETG00000 } \\
034354\end{array}$ & nek11 & -1.20 & 1.39E-03 \\
\hline $\begin{array}{l}\text { ENSXETG00000 } \\
006172\end{array}$ & c6orf165 & -1.20 & 4.60E-07 \\
\hline $\begin{array}{l}\text { ENSXETG00000 } \\
007394\end{array}$ & c10orf140 & -1.20 & 1.63E-02 \\
\hline $\begin{array}{l}\text { ENSXETG00000 } \\
030283\end{array}$ & $f g d 1$ & -1.20 & 6.07E-05 \\
\hline $\begin{array}{l}\text { ENSXETG00000 } \\
017882\end{array}$ & $\mathrm{fb} \times 043$ & -1.20 & $2.39 \mathrm{E}-06$ \\
\hline $\begin{array}{l}\text { ENSXETG00000 } \\
009988\end{array}$ & & -1.20 & 4.46E-02 \\
\hline $\begin{array}{l}\text { ENSXETG00000 } \\
013759\end{array}$ & ccdc135 & -1.20 & $1.41 \mathrm{E}-05$ \\
\hline $\begin{array}{l}\text { ENSXETG00000 } \\
030281\end{array}$ & sntb2 & -1.20 & $2.07 \mathrm{E}-08$ \\
\hline $\begin{array}{l}\text { ENSXETG00000 } \\
023804\end{array}$ & gfra1 & -1.19 & $3.01 \mathrm{E}-02$ \\
\hline $\begin{array}{l}\text { ENSXETG00000 } \\
016145\end{array}$ & ccdc157 & -1.19 & $4.21 \mathrm{E}-05$ \\
\hline $\begin{array}{l}\text { ENSXETG00000 } \\
033712\end{array}$ & fry & -1.19 & $3.31 \mathrm{E}-03$ \\
\hline $\begin{array}{l}\text { ENSXETG00000 } \\
009697\end{array}$ & c20orf26 & -1.19 & $9.83 \mathrm{E}-03$ \\
\hline $\begin{array}{l}\text { ENSXETG00000 } \\
008640\end{array}$ & ctbs & -1.19 & $1.58 \mathrm{E}-02$ \\
\hline $\begin{array}{l}\text { ENSXETG00000 } \\
023191\end{array}$ & fam83a & -1.19 & $2.91 \mathrm{E}-02$ \\
\hline $\begin{array}{l}\text { ENSXETG00000 } \\
006027\end{array}$ & $\begin{array}{l}x b \text {-gene- } \\
5818700\end{array}$ & -1.19 & $1.66 \mathrm{E}-02$ \\
\hline $\begin{array}{l}\text { ENSXETG00000 } \\
003627\end{array}$ & wdr9o & -1.19 & $4.85 \mathrm{E}-10$ \\
\hline $\begin{array}{l}\text { ENSXETG00000 } \\
031278\end{array}$ & & -1.18 & $1.14 \mathrm{E}-03$ \\
\hline
\end{tabular}




\begin{tabular}{|c|c|c|c|}
\hline $\begin{array}{l}\text { ENSXETG00000 } \\
009994\end{array}$ & mусьрар & -1.18 & 1.86E-04 \\
\hline $\begin{array}{l}\text { ENSXETG00000 } \\
016761\end{array}$ & сер19 & -1.18 & 1.16E-02 \\
\hline $\begin{array}{l}\text { ENSXETG00000 } \\
033547\end{array}$ & & -1.18 & $3.25 \mathrm{E}-02$ \\
\hline $\begin{array}{l}\text { ENSXETG00000 } \\
019549\end{array}$ & foxj1 & -1.18 & $2.77 \mathrm{E}-06$ \\
\hline $\begin{array}{l}\text { ENSXETG00000 } \\
030692\end{array}$ & $\operatorname{sh} 3 t c 2$ & -1.18 & 3.87E-02 \\
\hline $\begin{array}{l}\text { ENSXETG00000 } \\
023988\end{array}$ & zfpm1 & -1.18 & 4.71E-04 \\
\hline $\begin{array}{l}\text { ENSXETG00000 } \\
020484\end{array}$ & zfyve28 & -1.18 & 4.37E-02 \\
\hline $\begin{array}{l}\text { ENSXETG00000 } \\
001893\end{array}$ & c12orf55 & -1.18 & 3.86E-02 \\
\hline $\begin{array}{l}\text { ENSXETG00000 } \\
008804\end{array}$ & atf5.2 & -1.18 & 2.02E-02 \\
\hline $\begin{array}{l}\text { ENSXETG00000 } \\
002166\end{array}$ & dnali1 & -1.18 & $2.26 \mathrm{E}-04$ \\
\hline $\begin{array}{l}\text { ENSXETG00000 } \\
016124\end{array}$ & alcam & -1.18 & $5.01 \mathrm{E}-03$ \\
\hline $\begin{array}{l}\text { ENSXETG00000 } \\
009215\end{array}$ & Irrc31 & -1.18 & 1.13E-02 \\
\hline $\begin{array}{l}\text { ENSXETG00000 } \\
006170\end{array}$ & $\operatorname{ccdc} 13$ & -1.17 & $1.84 \mathrm{E}-02$ \\
\hline $\begin{array}{l}\text { ENSXETG00000 } \\
013405\end{array}$ & mapk15 & -1.17 & 5.67E-07 \\
\hline $\begin{array}{l}\text { ENSXETG00000 } \\
004564\end{array}$ & fut1 & -1.17 & $9.55 \mathrm{E}-03$ \\
\hline $\begin{array}{l}\text { ENSXETG00000 } \\
017730\end{array}$ & wdr93 & -1.17 & $2.46 \mathrm{E}-02$ \\
\hline $\begin{array}{l}\text { ENSXETG00000 } \\
013740\end{array}$ & ca2 & -1.17 & $1.42 \mathrm{E}-02$ \\
\hline $\begin{array}{l}\text { ENSXETG00000 } \\
023881\end{array}$ & kif9 & -1.17 & $1.09 \mathrm{E}-06$ \\
\hline $\begin{array}{l}\text { ENSXETG00000 } \\
022522\end{array}$ & plk4 & -1.16 & 1.43E-09 \\
\hline $\begin{array}{l}\text { ENSXETG00000 } \\
001302\end{array}$ & noxred1 & -1.16 & 1.09E-02 \\
\hline $\begin{array}{l}\text { ENSXETG00000 } \\
019671\end{array}$ & cep76 & -1.16 & $7.08 \mathrm{E}-05$ \\
\hline $\begin{array}{l}\text { ENSXETG00000 } \\
000715\end{array}$ & hoxa1 & -1.16 & $1.27 \mathrm{E}-02$ \\
\hline $\begin{array}{l}\text { ENSXETG00000 } \\
001588\end{array}$ & $b b s 12$ & -1.16 & $5.58 \mathrm{E}-04$ \\
\hline $\begin{array}{l}\text { ENSXETG00000 } \\
005936\end{array}$ & fam126a & -1.16 & $3.35 \mathrm{E}-03$ \\
\hline $\begin{array}{l}\text { ENSXETG00000 } \\
006579\end{array}$ & $t b \times 3$ & -1.16 & $3.00 \mathrm{E}-02$ \\
\hline $\begin{array}{l}\text { ENSXETG00000 } \\
009392\end{array}$ & fam160a1 & -1.15 & $2.64 \mathrm{E}-02$ \\
\hline $\begin{array}{l}\text { ENSXETG00000 } \\
018333\end{array}$ & kiaa0556 & -1.15 & 2.84E-04 \\
\hline $\begin{array}{l}\text { ENSXETG00000 } \\
029925\end{array}$ & & -1.15 & $1.57 \mathrm{E}-02$ \\
\hline $\begin{array}{l}\text { ENSXETG00000 } \\
013734\end{array}$ & atp6v0d2 & -1.15 & $6.65 \mathrm{E}-04$ \\
\hline $\begin{array}{l}\text { ENSXETG00000 } \\
005517\end{array}$ & fermt1 & -1.15 & $4.18 \mathrm{E}-02$ \\
\hline $\begin{array}{l}\text { ENSXETG00000 } \\
022616\end{array}$ & fam184a & -1.15 & 6.17E-03 \\
\hline $\begin{array}{l}\text { ENSXETG00000 } \\
004200\end{array}$ & & -1.15 & $1.41 \mathrm{E}-02$ \\
\hline $\begin{array}{l}\text { ENSXETG00000 } \\
021413\end{array}$ & tpp1 & -1.15 & 3.34E-02 \\
\hline $\begin{array}{l}\text { ENSXETG00000 } \\
018202\end{array}$ & fam $228 b$ & -1.15 & 3.83E-02 \\
\hline $\begin{array}{l}\text { ENSXETG00000 } \\
022999\end{array}$ & ccdc18 & -1.14 & $2.89 \mathrm{E}-02$ \\
\hline $\begin{array}{l}\text { ENSXETG00000 } \\
012882\end{array}$ & meis2 & -1.14 & 1.19E-02 \\
\hline $\begin{array}{l}\text { ENSXETG00000 } \\
031289\end{array}$ & & -1.14 & 1.06E-02 \\
\hline
\end{tabular}




\begin{tabular}{|c|c|c|c|}
\hline $\begin{array}{l}\text { ENSXETG00000 } \\
031455\end{array}$ & ift88 & -1.14 & 3.60E-06 \\
\hline $\begin{array}{l}\text { ENSXETG00000 } \\
003366\end{array}$ & $r a b 27 b$ & -1.14 & 1.74E-02 \\
\hline $\begin{array}{l}\text { ENSXETG00000 } \\
032327\end{array}$ & ccdc92 & -1.14 & 1.57E-04 \\
\hline $\begin{array}{l}\text { ENSXETG00000 } \\
031527\end{array}$ & ccdc87 & -1.13 & $3.85 \mathrm{E}-03$ \\
\hline $\begin{array}{l}\text { ENSXETG00000 } \\
001384\end{array}$ & aadac/4 & -1.13 & 8.16E-03 \\
\hline $\begin{array}{l}\text { ENSXETG00000 } \\
010779\end{array}$ & spata4 & -1.13 & 7.30E-07 \\
\hline $\begin{array}{l}\text { ENSXETG00000 } \\
030332\end{array}$ & kiaa1407 & -1.13 & $6.22 \mathrm{E}-03$ \\
\hline $\begin{array}{l}\text { ENSXETG00000 } \\
012541\end{array}$ & dnajb13 & -1.13 & 7.30E-07 \\
\hline $\begin{array}{l}\text { ENSXETG00000 } \\
008955\end{array}$ & acaca & -1.13 & 3.07E-02 \\
\hline $\begin{array}{l}\text { ENSXETG00000 } \\
015924\end{array}$ & Irrk1 & -1.13 & 2.03E-02 \\
\hline $\begin{array}{l}\text { ENSXETG00000 } \\
013007\end{array}$ & ugp2 & -1.13 & 1.30E-05 \\
\hline $\begin{array}{l}\text { ENSXETG00000 } \\
009728\end{array}$ & usp3 & -1.13 & $3.28 \mathrm{E}-05$ \\
\hline $\begin{array}{l}\text { ENSXETG00000 } \\
004420\end{array}$ & ccdc89 & -1.13 & $1.50 \mathrm{E}-02$ \\
\hline $\begin{array}{l}\text { ENSXETG00000 } \\
006535\end{array}$ & prickle2 & -1.12 & $6.21 \mathrm{E}-04$ \\
\hline $\begin{array}{l}\text { ENSXETG00000 } \\
025336\end{array}$ & cxorf58 & -1.12 & 1.33E-02 \\
\hline $\begin{array}{l}\text { ENSXETG00000 } \\
003329\end{array}$ & ccdc164 & -1.12 & $3.28 \mathrm{E}-03$ \\
\hline $\begin{array}{l}\text { ENSXETG00000 } \\
031946\end{array}$ & dennd1b & -1.12 & $2.90 \mathrm{E}-02$ \\
\hline $\begin{array}{l}\text { ENSXETG00000 } \\
024543\end{array}$ & tex9 & -1.12 & $2.65 E-05$ \\
\hline $\begin{array}{l}\text { ENSXETG00000 } \\
001355\end{array}$ & $n c s 1$ & -1.12 & $9.94 \mathrm{E}-04$ \\
\hline $\begin{array}{l}\text { ENSXETG00000 } \\
004106\end{array}$ & $f z d 6$ & -1.12 & $1.06 \mathrm{E}-03$ \\
\hline $\begin{array}{l}\text { ENSXETG00000 } \\
023039\end{array}$ & Irrc6 & -1.11 & 1.67E-02 \\
\hline $\begin{array}{l}\text { ENSXETG00000 } \\
016749\end{array}$ & eppk1 & -1.11 & $5.30 \mathrm{E}-03$ \\
\hline $\begin{array}{l}\text { ENSXETG00000 } \\
024456\end{array}$ & $\mathrm{fb} \times 046$ & -1.11 & $8.98 \mathrm{E}-04$ \\
\hline $\begin{array}{l}\text { ENSXETG00000 } \\
009961\end{array}$ & kcnk5 & -1.11 & $8.64 \mathrm{E}-03$ \\
\hline $\begin{array}{l}\text { ENSXETG00000 } \\
004670\end{array}$ & $m v p$ & -1.11 & $2.28 \mathrm{E}-05$ \\
\hline $\begin{array}{l}\text { ENSXETG00000 } \\
031364\end{array}$ & $n s f$ & -1.11 & 1.40E-02 \\
\hline $\begin{array}{l}\text { ENSXETG00000 } \\
019251\end{array}$ & tubala & -1.11 & $5.42 \mathrm{E}-07$ \\
\hline $\begin{array}{l}\text { ENSXETG00000 } \\
010700\end{array}$ & ccdc83 & -1.11 & $4.29 \mathrm{E}-02$ \\
\hline $\begin{array}{l}\text { ENSXETG00000 } \\
009216\end{array}$ & Irriq4 & -1.11 & $2.62 \mathrm{E}-03$ \\
\hline $\begin{array}{l}\text { ENSXETG00000 } \\
024111\end{array}$ & casp6 & -1.11 & $1.41 \mathrm{E}-03$ \\
\hline $\begin{array}{l}\text { ENSXETG00000 } \\
012423\end{array}$ & scnn1g & -1.11 & 4.68E-02 \\
\hline $\begin{array}{l}\text { ENSXETG00000 } \\
020096\end{array}$ & siah2 & -1.11 & 1.83E-02 \\
\hline $\begin{array}{l}\text { ENSXETG00000 } \\
019437\end{array}$ & ccdc39 & -1.11 & 4.28E-06 \\
\hline $\begin{array}{l}\text { ENSXETG00000 } \\
009902\end{array}$ & c21orf59 & -1.10 & $1.91 \mathrm{E}-07$ \\
\hline $\begin{array}{l}\text { ENSXETG00000 } \\
016769\end{array}$ & hs6st1 & -1.10 & $2.91 \mathrm{E}-04$ \\
\hline $\begin{array}{l}\text { ENSXETG00000 } \\
018842\end{array}$ & enkur & -1.10 & $4.25 \mathrm{E}-05$ \\
\hline $\begin{array}{l}\text { ENSXETG00000 } \\
010315\end{array}$ & ppp4r4 & -1.10 & $1.56 \mathrm{E}-04$ \\
\hline
\end{tabular}




\begin{tabular}{|c|c|c|c|}
\hline $\begin{array}{l}\text { ENSXETG00000 } \\
021254\end{array}$ & ift172 & -1.10 & 1.59E-04 \\
\hline $\begin{array}{l}\text { ENSXETG00000 } \\
032001\end{array}$ & bicd1 & -1.10 & $3.80 \mathrm{E}-02$ \\
\hline $\begin{array}{l}\text { ENSXETG00000 } \\
016745\end{array}$ & ccdc151 & -1.10 & $1.76 \mathrm{E}-03$ \\
\hline $\begin{array}{l}\text { ENSXETG00000 } \\
020238\end{array}$ & $a g b / 3$ & -1.10 & $1.20 \mathrm{E}-02$ \\
\hline $\begin{array}{l}\text { ENSXETG00000 } \\
033274\end{array}$ & mcidas & -1.10 & $1.91 \mathrm{E}-03$ \\
\hline $\begin{array}{l}\text { ENSXETG00000 } \\
009178\end{array}$ & c9orf93 & -1.10 & 2.63E-06 \\
\hline $\begin{array}{l}\text { ENSXETG00000 } \\
020322\end{array}$ & $\operatorname{ccdc11}$ & -1.10 & $1.72 \mathrm{E}-05$ \\
\hline $\begin{array}{l}\text { ENSXETG00000 } \\
002073\end{array}$ & c19orf44 & -1.09 & $6.09 \mathrm{E}-05$ \\
\hline $\begin{array}{l}\text { ENSXETG00000 } \\
004663\end{array}$ & & -1.09 & 1.40E-02 \\
\hline $\begin{array}{l}\text { ENSXETG00000 } \\
002225\end{array}$ & $\operatorname{rod} 1$ & -1.09 & $6.26 \mathrm{E}-03$ \\
\hline $\begin{array}{l}\text { ENSXETG00000 } \\
005963\end{array}$ & tmprss2 & -1.09 & $3.04 \mathrm{E}-03$ \\
\hline $\begin{array}{l}\text { ENSXETG00000 } \\
020468\end{array}$ & ppargc1a & -1.09 & 8.43E-04 \\
\hline $\begin{array}{l}\text { ENSXETG00000 } \\
021069\end{array}$ & сер135 & -1.09 & $2.69 \mathrm{E}-04$ \\
\hline $\begin{array}{l}\text { ENSXETG00000 } \\
028091\end{array}$ & ccno & -1.08 & $6.74 \mathrm{E}-03$ \\
\hline $\begin{array}{l}\text { ENSXETG00000 } \\
031645\end{array}$ & efnb3 & -1.08 & $5.98 \mathrm{E}-03$ \\
\hline $\begin{array}{l}\text { ENSXETG00000 } \\
010891\end{array}$ & tcte1 & -1.08 & $2.69 \mathrm{E}-03$ \\
\hline $\begin{array}{l}\text { ENSXETG00000 } \\
030937\end{array}$ & $f \operatorname{sip} 1$ & -1.08 & 1.86E-05 \\
\hline $\begin{array}{l}\text { ENSXETG00000 } \\
033876\end{array}$ & & -1.08 & $6.42 \mathrm{E}-03$ \\
\hline $\begin{array}{l}\text { ENSXETG00000 } \\
008997\end{array}$ & plekha8 & -1.08 & 2.03E-02 \\
\hline $\begin{array}{l}\text { ENSXETG00000 } \\
012970\end{array}$ & rap1gap2 & -1.08 & $1.89 \mathrm{E}-02$ \\
\hline $\begin{array}{l}\text { ENSXETG00000 } \\
011732\end{array}$ & agtpbp1 & -1.08 & $6.01 \mathrm{E}-03$ \\
\hline $\begin{array}{l}\text { ENSXETG00000 } \\
009217\end{array}$ & Irrc34 & -1.07 & 7.79E-05 \\
\hline $\begin{array}{l}\text { ENSXETG00000 } \\
025773\end{array}$ & c1orf222 & -1.07 & 1.15E-03 \\
\hline $\begin{array}{l}\text { ENSXETG00000 } \\
033996\end{array}$ & pcnt & -1.07 & $5.57 \mathrm{E}-03$ \\
\hline $\begin{array}{l}\text { ENSXETG00000 } \\
012328\end{array}$ & $u b \times n 11$ & -1.07 & $5.45 \mathrm{E}-03$ \\
\hline $\begin{array}{l}\text { ENSXETG00000 } \\
017447\end{array}$ & c1orf21 & -1.07 & $2.59 \mathrm{E}-04$ \\
\hline $\begin{array}{l}\text { ENSXETG00000 } \\
016556\end{array}$ & mns1 & -1.07 & $1.91 \mathrm{E}-03$ \\
\hline $\begin{array}{l}\text { ENSXETG00000 } \\
019096\end{array}$ & rsph6a & -1.07 & $9.66 \mathrm{E}-05$ \\
\hline $\begin{array}{l}\text { ENSXETG00000 } \\
001463\end{array}$ & ift122 & -1.06 & $3.26 \mathrm{E}-02$ \\
\hline $\begin{array}{l}\text { ENSXETG00000 } \\
025625\end{array}$ & agr2 & -1.06 & $1.55 \mathrm{E}-06$ \\
\hline $\begin{array}{l}\text { ENSXETG00000 } \\
018105\end{array}$ & micall2 & -1.06 & $9.61 \mathrm{E}-06$ \\
\hline $\begin{array}{l}\text { ENSXETG00000 } \\
008978\end{array}$ & styxl1 & -1.06 & 8.97E-09 \\
\hline $\begin{array}{l}\text { ENSXETG00000 } \\
013739\end{array}$ & ccdc113 & -1.06 & $4.01 \mathrm{E}-04$ \\
\hline $\begin{array}{l}\text { ENSXETG00000 } \\
014260\end{array}$ & fam129b & -1.05 & $2.20 \mathrm{E}-06$ \\
\hline $\begin{array}{l}\text { ENSXETG00000 } \\
006071\end{array}$ & ccdc104 & -1.05 & $1.58 \mathrm{E}-08$ \\
\hline $\begin{array}{l}\text { ENSXETG00000 } \\
009815\end{array}$ & ccdc65 & -1.05 & 3.14E-05 \\
\hline $\begin{array}{l}\text { ENSXETG00000 } \\
012155\end{array}$ & Irrc48 & -1.05 & 7.42E-03 \\
\hline
\end{tabular}




\begin{tabular}{|c|c|c|c|}
\hline $\begin{array}{l}\text { ENSXETG00000 } \\
001229\end{array}$ & $m g c 79783$ & -1.05 & $1.85 \mathrm{E}-02$ \\
\hline $\begin{array}{l}\text { ENSXETG00000 } \\
018573\end{array}$ & foxn4 & -1.05 & $7.60 \mathrm{E}-03$ \\
\hline $\begin{array}{l}\text { ENSXETG00000 } \\
018017\end{array}$ & gpr156 & -1.04 & $2.45 \mathrm{E}-02$ \\
\hline $\begin{array}{l}\text { ENSXETG00000 } \\
023264\end{array}$ & cspp1 & -1.03 & $3.00 \mathrm{E}-02$ \\
\hline $\begin{array}{l}\text { ENSXETG00000 } \\
013130\end{array}$ & magi1 & -1.03 & 5.27E-03 \\
\hline $\begin{array}{l}\text { ENSXETG00000 } \\
001660\end{array}$ & $\begin{array}{l}\text { xb-gene- } \\
5815363\end{array}$ & -1.03 & $1.68 \mathrm{E}-02$ \\
\hline $\begin{array}{l}\text { ENSXETG00000 } \\
000979\end{array}$ & $t t b k 2$ & -1.03 & $2.61 \mathrm{E}-02$ \\
\hline $\begin{array}{l}\text { ENSXETG00000 } \\
007807\end{array}$ & iqch & -1.03 & $1.02 \mathrm{E}-03$ \\
\hline $\begin{array}{l}\text { ENSXETG00000 } \\
020472\end{array}$ & pacrgl & -1.03 & 2.07E-02 \\
\hline $\begin{array}{l}\text { ENSXETG00000 } \\
000702\end{array}$ & ttc23l & -1.02 & 7.11E-05 \\
\hline $\begin{array}{l}\text { ENSXETG00000 } \\
017228\end{array}$ & rnasek & -1.02 & 8.47E-05 \\
\hline $\begin{array}{l}\text { ENSXETG00000 } \\
004733\end{array}$ & kifap3 & -1.02 & $3.20 \mathrm{E}-02$ \\
\hline $\begin{array}{l}\text { ENSXETG00000 } \\
003223\end{array}$ & esyt3 & -1.02 & 4.47E-03 \\
\hline $\begin{array}{l}\text { ENSXETG00000 } \\
034059\end{array}$ & & -1.02 & $2.22 \mathrm{E}-04$ \\
\hline $\begin{array}{l}\text { ENSXETG00000 } \\
033349\end{array}$ & $\operatorname{mgc14} 7546$ & -1.02 & $2.66 \mathrm{E}-04$ \\
\hline $\begin{array}{l}\text { ENSXETG00000 } \\
019937\end{array}$ & $a g b / 2$ & -1.02 & $2.16 \mathrm{E}-02$ \\
\hline $\begin{array}{l}\text { ENSXETG00000 } \\
016014\end{array}$ & stom/3 & -1.02 & $8.64 \mathrm{E}-03$ \\
\hline $\begin{array}{l}\text { ENSXETG00000 } \\
002292\end{array}$ & acly & -1.02 & 1.92E-04 \\
\hline $\begin{array}{l}\text { ENSXETG00000 } \\
012554\end{array}$ & capn7 & -1.02 & $2.15 \mathrm{E}-04$ \\
\hline $\begin{array}{l}\text { ENSXETG00000 } \\
007726\end{array}$ & plp2 & -1.01 & 1.50E-02 \\
\hline $\begin{array}{l}\text { ENSXETG00000 } \\
030278\end{array}$ & adam7 & -1.01 & $1.15 \mathrm{E}-03$ \\
\hline $\begin{array}{l}\text { ENSXETG00000 } \\
008635\end{array}$ & ssx2ip & -1.01 & $6.12 \mathrm{E}-03$ \\
\hline $\begin{array}{l}\text { ENSXETG00000 } \\
016097\end{array}$ & pgp & -1.01 & $1.95 \mathrm{E}-03$ \\
\hline $\begin{array}{l}\text { ENSXETG00000 } \\
014740\end{array}$ & arhgap23 & -1.01 & $1.01 \mathrm{E}-02$ \\
\hline $\begin{array}{l}\text { ENSXETG00000 } \\
015225\end{array}$ & arl3.1 & -1.01 & $7.82 \mathrm{E}-08$ \\
\hline $\begin{array}{l}\text { ENSXETG00000 } \\
021305\end{array}$ & nbeal2 & -1.01 & $2.38 \mathrm{E}-02$ \\
\hline $\begin{array}{l}\text { ENSXETG00000 } \\
014645\end{array}$ & slc4a11 & -1.00 & $5.41 \mathrm{E}-03$ \\
\hline $\begin{array}{l}\text { ENSXETG00000 } \\
031231\end{array}$ & $f b f 1$ & -1.00 & $3.12 \mathrm{E}-03$ \\
\hline $\begin{array}{l}\text { ENSXETG00000 } \\
018458\end{array}$ & plcd1 & -1.00 & $1.45 \mathrm{E}-02$ \\
\hline $\begin{array}{l}\text { ENSXETG00000 } \\
000967\end{array}$ & nadsyn1 & -1.00 & $2.05 \mathrm{E}-03$ \\
\hline $\begin{array}{l}\text { ENSXETG00000 } \\
002421\end{array}$ & c1orf114 & -1.00 & $1.68 \mathrm{E}-04$ \\
\hline $\begin{array}{l}\text { ENSXETG00000 } \\
020945\end{array}$ & ift81 & -1.00 & $6.89 \mathrm{E}-04$ \\
\hline $\begin{array}{l}\text { ENSXETG00000 } \\
031398\end{array}$ & cсp110 & -1.00 & 3.74E-04 \\
\hline $\begin{array}{l}\text { ENSXETG00000 } \\
010380\end{array}$ & acvr1 & -1.00 & $7.74 \mathrm{E}-03$ \\
\hline
\end{tabular}




\subsubsection{Differentially expressed genes at stage 27}

Given are the ensembl id, official gene name, log2 fold change and the adjusted P-value with respect to multiple testing using the FDR method. Genes were sorted according to the highest and lowest log2 fold change, respectively.

Table 6.2 Summary of differentially expressed genes at stage 27

\begin{tabular}{|c|c|c|c|}
\hline \multicolumn{4}{|c|}{ upregulated } \\
\hline ensembl id & Gene & \begin{tabular}{|l|} 
log2 \\
Fold \\
Change \\
\end{tabular} & $\begin{array}{l}\text { adjust. } \\
\text { P-value } \\
\end{array}$ \\
\hline $\begin{array}{l}\text { ENSXETG00000 } \\
006348\end{array}$ & sox10 & 7.92 & 7.84E-21 \\
\hline $\begin{array}{l}\text { ENSXETG00000 } \\
023476\end{array}$ & hoxc8 & 7.56 & 1.34E-37 \\
\hline $\begin{array}{l}\text { ENSXETG00000 } \\
015915\end{array}$ & prdm14 & 7.44 & 1.19E-33 \\
\hline $\begin{array}{l}\text { ENSXETG00000 } \\
007863\end{array}$ & stmn2 & 7.25 & 5.73E-11 \\
\hline $\begin{array}{l}\text { ENSXETG00000 } \\
005657\end{array}$ & penk & 6.80 & $3.34 \mathrm{E}-10$ \\
\hline $\begin{array}{l}\text { ENSXETG00000 } \\
023475\end{array}$ & hoxc9 & 6.65 & 7.84E-21 \\
\hline $\begin{array}{l}\text { ENSXETG00000 } \\
003618\end{array}$ & atp2b4 & 6.61 & 4.11E-10 \\
\hline $\begin{array}{l}\text { ENSXETG00000 } \\
016479\end{array}$ & trpm1 & 6.58 & $1.29 \mathrm{E}-10$ \\
\hline $\begin{array}{l}\text { ENSXETG00000 } \\
023479\end{array}$ & hoxc6 & 6.40 & $2.40 \mathrm{E}-15$ \\
\hline $\begin{array}{l}\text { ENSXETG00000 } \\
010836\end{array}$ & foxd3 & 6.34 & $3.01 \mathrm{E}-10$ \\
\hline $\begin{array}{l}\text { ENSXETG00000 } \\
032598\end{array}$ & map1b & 6.34 & $1.85 \mathrm{E}-10$ \\
\hline $\begin{array}{l}\text { ENSXETG00000 } \\
027815\end{array}$ & vcan & 6.28 & 1.89E-09 \\
\hline $\begin{array}{l}\text { ENSXETG00000 } \\
004874\end{array}$ & & 6.24 & 1.60E-08 \\
\hline $\begin{array}{l}\text { ENSXETG00000 } \\
018387\end{array}$ & ednrb2 & 6.20 & 1.29E-08 \\
\hline $\begin{array}{l}\text { ENSXETG00000 } \\
022336\end{array}$ & olfm1 & 6.15 & 1.51E-09 \\
\hline $\begin{array}{l}\text { ENSXETG00000 } \\
015096\end{array}$ & snap25 & 6.14 & 1.07E-17 \\
\hline $\begin{array}{l}\text { ENSXETG00000 } \\
008965\end{array}$ & scn3a & 6.12 & 2.24E-10 \\
\hline $\begin{array}{l}\text { ENSXETG00000 } \\
003388\end{array}$ & adam11 & 5.97 & 3.77E-09 \\
\hline $\begin{array}{l}\text { ENSXETG00000 } \\
011738\end{array}$ & tyr & 5.91 & 2.04E-08 \\
\hline $\begin{array}{l}\text { ENSXETG00000 } \\
018304\end{array}$ & $t \mid x 3$ & 5.91 & 5.73E-11 \\
\hline $\begin{array}{l}\text { ENSXETG00000 } \\
030140\end{array}$ & $s t \times 1 b$ & 5.84 & 2.36E-07 \\
\hline $\begin{array}{l}\text { ENSXETG00000 } \\
003399\end{array}$ & slc17a7 & 5.78 & 4.18E-09 \\
\hline $\begin{array}{l}\text { ENSXETG00000 } \\
030402\end{array}$ & & 5.77 & 4.41E-07 \\
\hline $\begin{array}{l}\text { ENSXETG00000 } \\
000237\end{array}$ & zeb2 & 5.73 & 5.32E-09 \\
\hline $\begin{array}{l}\text { ENSXETG00000 } \\
019179\end{array}$ & adcyap1 & 5.68 & 4.49E-08 \\
\hline $\begin{array}{l}\text { ENSXETG00000 } \\
013347\end{array}$ & gpm6b & 5.62 & 1.03E-09 \\
\hline
\end{tabular}

\begin{tabular}{|c|c|c|c|}
\hline \multicolumn{4}{|c|}{ downregulated } \\
\hline ensembl id & Gene & $\begin{array}{l}\text { log2 } \\
\text { Fold } \\
\text { Change } \\
\end{array}$ & $\begin{array}{l}\text { adjust. } \\
\text { P-value }\end{array}$ \\
\hline $\begin{array}{l}\text { ENSXETG00000 } \\
032850\end{array}$ & Irrn3 & -2.69 & 8.13E-03 \\
\hline $\begin{array}{l}\text { ENSXETG00000 } \\
001728\end{array}$ & slc6a3 & -2.50 & 2.79E-02 \\
\hline $\begin{array}{l}\text { ENSXETG00000 } \\
032366\end{array}$ & $\lg 5$ & -2.49 & 7.86E-04 \\
\hline $\begin{array}{l}\text { ENSXETG00000 } \\
008859\end{array}$ & $h k d c 1$ & -2.16 & 1.27E-03 \\
\hline $\begin{array}{l}\text { ENSXETG00000 } \\
012512\end{array}$ & $\begin{array}{l}\text { xb-gene- } \\
990053\end{array}$ & -1.99 & 2.94E-04 \\
\hline $\begin{array}{l}\text { ENSXETG00000 } \\
001035\end{array}$ & fam132a & -1.93 & 1.45E-02 \\
\hline $\begin{array}{l}\text { ENSXETG00000 } \\
017075\end{array}$ & $p d e 8 b$ & -1.89 & 4.70E-02 \\
\hline $\begin{array}{l}\text { ENSXETG00000 } \\
027798\end{array}$ & pnmt & -1.88 & 2.89E-02 \\
\hline \multicolumn{2}{|c|}{ ENSXETG00000022857 } & -1.83 & $9.72 \mathrm{E}-03$ \\
\hline $\begin{array}{l}\text { ENSXETG00000 } \\
007353\end{array}$ & $\operatorname{tgm} 5$ & -1.78 & $6.28 \mathrm{E}-03$ \\
\hline $\begin{array}{l}\text { ENSXETG00000 } \\
031045\end{array}$ & sepp1 & -1.64 & 1.91E-02 \\
\hline $\begin{array}{l}\text { ENSXETG00000 } \\
021580\end{array}$ & slc2a7 & -1.61 & $2.48 \mathrm{E}-03$ \\
\hline $\begin{array}{l}\text { ENSXETG00000 } \\
014607\end{array}$ & calb1 & -1.60 & $1.15 \mathrm{E}-02$ \\
\hline $\begin{array}{l}\text { ENSXETG00000 } \\
004839\end{array}$ & maob & -1.56 & 4.89E-02 \\
\hline $\begin{array}{l}\text { ENSXETG00000 } \\
020701\end{array}$ & tpte2 & -1.56 & 2.91E-04 \\
\hline \multicolumn{2}{|c|}{ ENSXETG00000032779 } & -1.55 & 1.80E-04 \\
\hline $\begin{array}{l}\text { ENSXETG00000 } \\
032204\end{array}$ & plcl1 & -1.52 & 1.98E-02 \\
\hline $\begin{array}{l}\text { ENSXETG00000 } \\
020033\end{array}$ & serpinc1 & -1.51 & 8.88E-06 \\
\hline $\begin{array}{l}\text { ENSXETG00000 } \\
018739\end{array}$ & cdo1 & -1.49 & 4.41E-03 \\
\hline \multicolumn{2}{|c|}{ ENSXETG00000031991 } & -1.47 & 4.52E-05 \\
\hline $\begin{array}{l}\text { ENSXETG00000 } \\
033983\end{array}$ & dmrt2 & -1.47 & 3.72E-04 \\
\hline $\begin{array}{l}\text { ENSXETG00000 } \\
015904\end{array}$ & lctl & -1.46 & 1.20E-02 \\
\hline \multicolumn{2}{|c|}{ ENSXETG00000018800 } & -1.42 & $3.28 \mathrm{E}-02$ \\
\hline $\begin{array}{l}\text { ENSXETG00000 } \\
017719\end{array}$ & $p d e 7 b$ & -1.42 & $5.79 \mathrm{E}-04$ \\
\hline $\begin{array}{l}\text { ENSXETG00000 } \\
007364\end{array}$ & slc4a1 & -1.38 & 6.87E-04 \\
\hline $\begin{array}{l}\text { ENSXETG00000 } \\
002371\end{array}$ & kif27 & -1.36 & $2.12 \mathrm{E}-02$ \\
\hline
\end{tabular}




\begin{tabular}{|c|c|c|c|}
\hline $\begin{array}{l}\text { ENSXETG00000 } \\
000724\end{array}$ & hoxa9 & 5.56 & $1.29 \mathrm{E}-08$ \\
\hline $\begin{array}{l}\text { ENSXETG00000 } \\
020467\end{array}$ & cdh11 & 5.56 & $1.79 \mathrm{E}-08$ \\
\hline $\begin{array}{l}\text { ENSXETG00000 } \\
003657\end{array}$ & sncg & 5.56 & $1.20 \mathrm{E}-14$ \\
\hline $\begin{array}{l}\text { ENSXETG00000 } \\
019353\end{array}$ & cadm3 & 5.45 & $6.81 \mathrm{E}-06$ \\
\hline $\begin{array}{l}\text { ENSXETG00000 } \\
018040\end{array}$ & ntrk1 & 5.35 & 2.73E-06 \\
\hline $\begin{array}{l}\text { ENSXETG00000 } \\
002556\end{array}$ & slc45a2 & 5.35 & $1.29 \mathrm{E}-08$ \\
\hline $\begin{array}{l}\text { ENSXETG00000 } \\
020978\end{array}$ & robo2 & 5.34 & $3.74 \mathrm{E}-08$ \\
\hline $\begin{array}{l}\text { ENSXETG00000 } \\
000719\end{array}$ & I1cam & 5.32 & $2.27 \mathrm{E}-10$ \\
\hline $\begin{array}{l}\text { ENSXETG00000 } \\
011751\end{array}$ & rab38 & 5.31 & 4.37E-06 \\
\hline $\begin{array}{l}\text { ENSXETG00000 } \\
006932\end{array}$ & cdh2 & 5.29 & $2.71 \mathrm{E}-09$ \\
\hline $\begin{array}{l}\text { ENSXETG00000 } \\
006230\end{array}$ & kif5a & 5.14 & 7.99E-07 \\
\hline $\begin{array}{l}\text { ENSXETG00000 } \\
009943\end{array}$ & ntng1 & 5.14 & 1.82E-05 \\
\hline $\begin{array}{l}\text { ENSXETG00000 } \\
013534\end{array}$ & $r t n 4 r / 1$ & 5.11 & 9.82E-08 \\
\hline $\begin{array}{l}\text { ENSXETG00000 } \\
023938\end{array}$ & thc & 5.10 & 1.24E-09 \\
\hline $\begin{array}{l}\text { ENSXETG00000 } \\
021492\end{array}$ & Irfn5 & 5.08 & $2.01 \mathrm{E}-05$ \\
\hline $\begin{array}{l}\text { ENSXETG00000 } \\
010942\end{array}$ & $d c t$ & 5.07 & $6.40 \mathrm{E}-06$ \\
\hline $\begin{array}{l}\text { ENSXETG00000 } \\
015442\end{array}$ & syt4 & 5.05 & 1.71E-05 \\
\hline $\begin{array}{l}\text { ENSXETG00000 } \\
026846\end{array}$ & sncb & 5.04 & 1.75E-07 \\
\hline $\begin{array}{l}\text { ENSXETG00000 } \\
032855\end{array}$ & tmem196 & 5.02 & 4.07E-05 \\
\hline $\begin{array}{l}\text { ENSXETG00000 } \\
031363\end{array}$ & $r a b 6 b$ & 5.00 & $7.86 \mathrm{E}-08$ \\
\hline $\begin{array}{l}\text { ENSXETG00000 } \\
006562\end{array}$ & shc2 & 4.99 & 1.10E-05 \\
\hline $\begin{array}{l}\text { ENSXETG00000 } \\
005838\end{array}$ & mllt11 & 4.99 & 8.53E-06 \\
\hline $\begin{array}{l}\text { ENSXETG00000 } \\
010153\end{array}$ & ebf2 & 4.96 & 4.12E-06 \\
\hline $\begin{array}{l}\text { ENSXETG00000 } \\
009667\end{array}$ & c1orf216 & 4.91 & $1.24 \mathrm{E}-05$ \\
\hline $\begin{array}{l}\text { ENSXETG00000 } \\
032232\end{array}$ & mlph & 4.86 & 1.49E-05 \\
\hline $\begin{array}{l}\text { ENSXETG00000 } \\
023585\end{array}$ & dpys/4 & 4.85 & $2.94 \mathrm{E}-08$ \\
\hline $\begin{array}{l}\text { ENSXETG00000 } \\
034215\end{array}$ & sipa1 & 4.84 & $6.25 \mathrm{E}-05$ \\
\hline $\begin{array}{l}\text { ENSXETG00000 } \\
001025\end{array}$ & mapk8ip1 & 4.82 & 3.30E-06 \\
\hline $\begin{array}{l}\text { ENSXETG00000 } \\
007640\end{array}$ & pax3 & 4.82 & 1.65E-05 \\
\hline $\begin{array}{l}\text { ENSXETG00000 } \\
003477\end{array}$ & fgf13 & 4.81 & 1.13E-05 \\
\hline $\begin{array}{l}\text { ENSXETG00000 } \\
016741\end{array}$ & elavl3 & 4.81 & 6.64E-07 \\
\hline $\begin{array}{l}\text { ENSXETG00000 } \\
009037\end{array}$ & fam176b & 4.77 & $6.01 \mathrm{E}-05$ \\
\hline $\begin{array}{l}\text { ENSXETG00000 } \\
019618\end{array}$ & $n p t \times 1$ & 4.74 & $6.05 \mathrm{E}-10$ \\
\hline $\begin{array}{l}\text { ENSXETG00000 } \\
004068\end{array}$ & syt1 & 4.73 & $2.36 \mathrm{E}-04$ \\
\hline $\begin{array}{l}\text { ENSXETG00000 } \\
022924\end{array}$ & brsk1 & 4.73 & $2.57 \mathrm{E}-04$ \\
\hline $\begin{array}{l}\text { ENSXETG00000 } \\
014261\end{array}$ & stxbp1 & 4.69 & $2.36 \mathrm{E}-09$ \\
\hline $\begin{array}{l}\text { ENSXETG00000 } \\
030030\end{array}$ & kctd16 & 4.68 & $2.25 \mathrm{E}-04$ \\
\hline
\end{tabular}

\begin{tabular}{|c|c|c|c|}
\hline $\begin{array}{l}\text { ENSXETG00000 } \\
008892\end{array}$ & $\operatorname{irx} 4$ & -1.34 & 4.41E-03 \\
\hline $\begin{array}{l}\text { ENSXETG00000 } \\
010125\end{array}$ & tmprss13 & -1.29 & 4.31E-02 \\
\hline $\begin{array}{l}\text { ENSXETG00000 } \\
020335\end{array}$ & fgfbp2 & -1.28 & $2.71 \mathrm{E}-02$ \\
\hline $\begin{array}{l}\text { ENSXETG00000 } \\
032404\end{array}$ & axdnd1 & -1.28 & 2.43E-02 \\
\hline $\begin{array}{l}\text { ENSXETG00000 } \\
013722\end{array}$ & ephb1 & -1.27 & $1.16 \mathrm{E}-05$ \\
\hline $\begin{array}{l}\text { ENSXETG00000 } \\
000158\end{array}$ & $\begin{array}{l}x b \text {-gene- } \\
5822257\end{array}$ & -1.25 & $1.59 \mathrm{E}-02$ \\
\hline \multicolumn{2}{|c|}{ ENSXETG00000030875 } & -1.21 & $1.66 \mathrm{E}-03$ \\
\hline \multicolumn{2}{|c|}{ ENSXETG00000000901 } & -1.13 & 2.45E-03 \\
\hline $\begin{array}{l}\text { ENSXETG00000 } \\
000157\end{array}$ & ppp1r14a & -1.10 & $3.27 \mathrm{E}-03$ \\
\hline \multicolumn{2}{|c|}{ ENSXETG00000032488 } & -1.09 & 8.57E-03 \\
\hline $\begin{array}{l}\text { ENSXETG00000 } \\
001660\end{array}$ & $\begin{array}{l}x b \text {-gene- } \\
5815363\end{array}$ & -1.06 & 4.15E-02 \\
\hline $\begin{array}{l}\text { ENSXETG00000 } \\
009697\end{array}$ & c20orf26 & -1.06 & 4.10E-02 \\
\hline $\begin{array}{l}\text { ENSXETG00000 } \\
014867\end{array}$ & pltp & -1.05 & $5.90 \mathrm{E}-03$ \\
\hline $\begin{array}{l}\text { ENSXETG00000 } \\
020710\end{array}$ & nek5 & -1.01 & $3.59 \mathrm{E}-03$ \\
\hline $\begin{array}{l}\text { ENSXETG00000 } \\
024858\end{array}$ & сур2с18 & -1.00 & $1.07 \mathrm{E}-02$ \\
\hline
\end{tabular}




\begin{tabular}{|c|c|c|c|}
\hline $\begin{array}{l}\text { ENSXETG00000 } \\
022315\end{array}$ & $c p l \times 2$ & 4.66 & $1.29 \mathrm{E}-08$ \\
\hline $\begin{array}{l}\text { ENSXETG00000 } \\
000728\end{array}$ & hoxa10 & 4.65 & 3.94E-05 \\
\hline $\begin{array}{l}\text { ENSXETG00000 } \\
030920\end{array}$ & atp2b3 & 4.63 & 1.27E-11 \\
\hline $\begin{array}{l}\text { ENSXETG00000 } \\
015058\end{array}$ & klh/35 & 4.63 & 1.56E-04 \\
\hline $\begin{array}{l}\text { ENSXETG00000 } \\
021181\end{array}$ & pcdh9 & 4.61 & 4.83E-05 \\
\hline $\begin{array}{l}\text { ENSXETG00000 } \\
000722\end{array}$ & hoxa7 & 4.58 & 1.65E-09 \\
\hline $\begin{array}{l}\text { ENSXETG00000 } \\
025525\end{array}$ & pnhd & 4.51 & 1.13E-05 \\
\hline $\begin{array}{l}\text { ENSXETG00000 } \\
015810\end{array}$ & cntn1 & 4.50 & $6.57 \mathrm{E}-05$ \\
\hline $\begin{array}{l}\text { ENSXETG00000 } \\
015061\end{array}$ & map6 & 4.48 & 3.33E-04 \\
\hline $\begin{array}{l}\text { ENSXETG00000 } \\
018386\end{array}$ & pou4f1.2 & 4.47 & 4.39E-08 \\
\hline $\begin{array}{l}\text { ENSXETG00000 } \\
011339\end{array}$ & sez6/2 & 4.47 & 9.87E-06 \\
\hline $\begin{array}{l}\text { ENSXETG00000 } \\
000225\end{array}$ & kcnh1 & 4.44 & $6.92 \mathrm{E}-04$ \\
\hline $\begin{array}{l}\text { ENSXETG00000 } \\
007718\end{array}$ & syp & 4.42 & 1.33E-06 \\
\hline $\begin{array}{l}\text { ENSXETG00000 } \\
020282\end{array}$ & itga8 & 4.39 & $5.23 \mathrm{E}-04$ \\
\hline $\begin{array}{l}\text { ENSXETG00000 } \\
005746\end{array}$ & sox8 & 4.37 & $2.10 \mathrm{E}-05$ \\
\hline $\begin{array}{l}\text { ENSXETG00000 } \\
000910\end{array}$ & arl8a & 4.37 & 8.02E-09 \\
\hline $\begin{array}{l}\text { ENSXETG00000 } \\
002939\end{array}$ & $\operatorname{scg} 2$ & 4.35 & 1.95E-05 \\
\hline $\begin{array}{l}\text { ENSXETG00000 } \\
022304\end{array}$ & svop & 4.34 & 2.91E-04 \\
\hline $\begin{array}{l}\text { ENSXETG00000 } \\
007649\end{array}$ & c11orf87 & 4.34 & 3.00E-04 \\
\hline $\begin{array}{l}\text { ENSXETG00000 } \\
025183\end{array}$ & hoxc5 & 4.29 & $5.89 \mathrm{E}-15$ \\
\hline $\begin{array}{l}\text { ENSXETG00000 } \\
007626\end{array}$ & $s v 2 b$ & 4.28 & $1.66 \mathrm{E}-03$ \\
\hline $\begin{array}{l}\text { ENSXETG00000 } \\
006906\end{array}$ & $n p t x r$ & 4.25 & 7.19E-07 \\
\hline $\begin{array}{l}\text { ENSXETG00000 } \\
016503\end{array}$ & apba2 & 4.24 & $2.79 \mathrm{E}-07$ \\
\hline $\begin{array}{l}\text { ENSXETG00000 } \\
022780\end{array}$ & fli1 & 4.24 & 8.94E-04 \\
\hline $\begin{array}{l}\text { ENSXETG00000 } \\
010118\end{array}$ & adam23 & 4.23 & 6.01E-05 \\
\hline $\begin{array}{l}\text { ENSXETG00000 } \\
033967\end{array}$ & slc18a3 & 4.18 & 4.68E-04 \\
\hline $\begin{array}{l}\text { ENSXETG00000 } \\
015711\end{array}$ & grm7 & 4.18 & $1.12 \mathrm{E}-03$ \\
\hline $\begin{array}{l}\text { ENSXETG00000 } \\
020846\end{array}$ & scn1a & 4.16 & 4.76E-04 \\
\hline $\begin{array}{l}\text { ENSXETG00000 } \\
008680\end{array}$ & elavl4 & 4.15 & 5.01E-07 \\
\hline $\begin{array}{l}\text { ENSXETG00000 } \\
002245\end{array}$ & frmpd1 & 4.14 & 2.06E-04 \\
\hline $\begin{array}{l}\text { ENSXETG00000 } \\
023482\end{array}$ & bhlhe41 & 4.13 & $6.73 \mathrm{E}-04$ \\
\hline $\begin{array}{l}\text { ENSXETG00000 } \\
001353\end{array}$ & hmcn2 & 4.13 & 4.38E-07 \\
\hline $\begin{array}{l}\text { ENSXETG00000 } \\
012179\end{array}$ & $r t n 4 r$ & 4.10 & $2.27 \mathrm{E}-03$ \\
\hline $\begin{array}{l}\text { ENSXETG00000 } \\
006793\end{array}$ & pdzrn4 & 4.09 & $1.56 \mathrm{E}-03$ \\
\hline $\begin{array}{l}\text { ENSXETG00000 } \\
022950\end{array}$ & zbtb16 & 4.09 & $6.37 \mathrm{E}-16$ \\
\hline $\begin{array}{l}\text { ENSXETG00000 } \\
025936\end{array}$ & cnrip1 & 4.05 & 4.52E-04 \\
\hline $\begin{array}{l}\text { ENSXETG00000 } \\
027666\end{array}$ & tmeff1 & 4.05 & 3.34E-10 \\
\hline
\end{tabular}




\begin{tabular}{|c|c|c|c|}
\hline $\begin{array}{l}\text { ENSXETG00000 } \\
010674\end{array}$ & atp8a2 & 4.04 & $1.52 \mathrm{E}-03$ \\
\hline $\begin{array}{l}\text { ENSXETG00000 } \\
034346\end{array}$ & dpys/2 & 4.04 & $6.29 \mathrm{E}-07$ \\
\hline $\begin{array}{l}\text { ENSXETG00000 } \\
000742\end{array}$ & $\operatorname{Im} \times 1 b .1$ & 4.00 & 4.22E-07 \\
\hline $\begin{array}{l}\text { ENSXETG00000 } \\
004296\end{array}$ & $\begin{array}{l}\text { xb-gene- } \\
987190\end{array}$ & 4.00 & 1.18E-03 \\
\hline $\begin{array}{l}\text { ENSXETG00000 } \\
001812\end{array}$ & postn & 3.99 & 4.62E-03 \\
\hline $\begin{array}{l}\text { ENSXETG00000 } \\
032363\end{array}$ & pou4f3 & 3.98 & 4.03E-03 \\
\hline $\begin{array}{l}\text { ENSXETG00000 } \\
000108\end{array}$ & c6orf192 & 3.96 & 3.59E-03 \\
\hline $\begin{array}{l}\text { ENSXETG00000 } \\
004406\end{array}$ & & 3.96 & 3.47E-05 \\
\hline $\begin{array}{l}\text { ENSXETG00000 } \\
006083\end{array}$ & slc1a3 & 3.92 & 1.12E-06 \\
\hline $\begin{array}{l}\text { ENSXETG00000 } \\
007492\end{array}$ & kcnj12 & 3.92 & 4.64E-03 \\
\hline $\begin{array}{l}\text { ENSXETG00000 } \\
009344\end{array}$ & kank2 & 3.90 & 4.39E-03 \\
\hline $\begin{array}{l}\text { ENSXETG00000 } \\
025170\end{array}$ & gpr173 & 3.89 & $5.75 \mathrm{E}-03$ \\
\hline $\begin{array}{l}\text { ENSXETG00000 } \\
026334\end{array}$ & cacnb1 & 3.88 & 1.23E-04 \\
\hline $\begin{array}{l}\text { ENSXETG00000 } \\
021973\end{array}$ & hoxb4 & 3.87 & $6.89 \mathrm{E}-04$ \\
\hline $\begin{array}{l}\text { ENSXETG00000 } \\
005205\end{array}$ & parp6 & 3.87 & $1.50 \mathrm{E}-03$ \\
\hline $\begin{array}{l}\text { ENSXETG00000 } \\
020236\end{array}$ & rbfox3 & 3.86 & $3.11 \mathrm{E}-04$ \\
\hline $\begin{array}{l}\text { ENSXETG00000 } \\
030565\end{array}$ & $\operatorname{drg} x$ & 3.86 & 1.56E-04 \\
\hline $\begin{array}{l}\text { ENSXETG00000 } \\
021993\end{array}$ & hoxb8 & 3.85 & $1.81 \mathrm{E}-03$ \\
\hline $\begin{array}{l}\text { ENSXETG00000 } \\
021195\end{array}$ & & 3.81 & $2.51 \mathrm{E}-04$ \\
\hline $\begin{array}{l}\text { ENSXETG00000 } \\
006675\end{array}$ & smpd3 & 3.80 & 3.27E-05 \\
\hline $\begin{array}{l}\text { ENSXETG00000 } \\
012718\end{array}$ & dpys/3 & 3.80 & 8.12E-11 \\
\hline $\begin{array}{l}\text { ENSXETG00000 } \\
022629\end{array}$ & snap91 & 3.78 & 4.07E-05 \\
\hline $\begin{array}{l}\text { ENSXETG00000 } \\
012629\end{array}$ & nrp1 & 3.76 & $1.52 \mathrm{E}-04$ \\
\hline $\begin{array}{l}\text { ENSXETG00000 } \\
017979\end{array}$ & $a v p$ & 3.75 & $9.78 \mathrm{E}-03$ \\
\hline $\begin{array}{l}\text { ENSXETG00000 } \\
017099\end{array}$ & kif21b & 3.75 & 7.73E-03 \\
\hline $\begin{array}{l}\text { ENSXETG00000 } \\
003405\end{array}$ & slit1 & 3.74 & 4.74E-05 \\
\hline $\begin{array}{l}\text { ENSXETG00000 } \\
020230\end{array}$ & hs3st5 & 3.73 & $6.22 \mathrm{E}-03$ \\
\hline $\begin{array}{l}\text { ENSXETG00000 } \\
015595\end{array}$ & npr3 & 3.73 & 8.99E-03 \\
\hline $\begin{array}{l}\text { ENSXETG00000 } \\
032535\end{array}$ & $f b n 2$ & 3.71 & 1.12E-03 \\
\hline $\begin{array}{l}\text { ENSXETG00000 } \\
011744\end{array}$ & mgat4c & 3.71 & 1.11E-02 \\
\hline $\begin{array}{l}\text { ENSXETG00000 } \\
014639\end{array}$ & palm & 3.69 & 8.48E-08 \\
\hline $\begin{array}{l}\text { ENSXETG00000 } \\
019847\end{array}$ & $d b n d d 1$ & 3.69 & $1.09 \mathrm{E}-02$ \\
\hline $\begin{array}{l}\text { ENSXETG00000 } \\
003565\end{array}$ & foxf1 & 3.67 & $3.68 \mathrm{E}-04$ \\
\hline $\begin{array}{l}\text { ENSXETG00000 } \\
014625\end{array}$ & nell2 & 3.66 & 7.69E-05 \\
\hline $\begin{array}{l}\text { ENSXETG00000 } \\
010136\end{array}$ & homer3 & 3.66 & $3.26 \mathrm{E}-03$ \\
\hline $\begin{array}{l}\text { ENSXETG00000 } \\
013321\end{array}$ & phlda1 & 3.65 & $1.20 \mathrm{E}-03$ \\
\hline $\begin{array}{l}\text { ENSXETG00000 } \\
025361\end{array}$ & $\begin{array}{l}x b-g e n e- \\
5911701 \\
\end{array}$ & 3.65 & $3.59 \mathrm{E}-03$ \\
\hline
\end{tabular}




\begin{tabular}{|c|c|c|c|}
\hline $\begin{array}{l}\text { ENSXETG00000 } \\
022236\end{array}$ & tspan18 & 3.62 & 1.43E-02 \\
\hline $\begin{array}{l}\text { ENSXETG00000 } \\
032009\end{array}$ & kcnq2 & 3.61 & $3.91 \mathrm{E}-03$ \\
\hline $\begin{array}{l}\text { ENSXETG00000 } \\
009439\end{array}$ & pmel & 3.60 & 1.19E-04 \\
\hline $\begin{array}{l}\text { ENSXETG00000 } \\
003071\end{array}$ & $f b / n 1$ & 3.59 & 1.10E-05 \\
\hline $\begin{array}{l}\text { ENSXETG00000 } \\
031277\end{array}$ & spry3 & 3.58 & $9.78 \mathrm{E}-03$ \\
\hline $\begin{array}{l}\text { ENSXETG00000 } \\
009191\end{array}$ & trim63 & 3.57 & 1.04E-02 \\
\hline $\begin{array}{l}\text { ENSXETG00000 } \\
022008\end{array}$ & $\operatorname{sh} 2 d 3 c$ & 3.56 & 2.32E-03 \\
\hline $\begin{array}{l}\text { ENSXETG00000 } \\
033300\end{array}$ & gareml & 3.55 & 1.74E-02 \\
\hline $\begin{array}{l}\text { ENSXETG00000 } \\
011309\end{array}$ & ebf3 & 3.55 & $2.57 \mathrm{E}-05$ \\
\hline $\begin{array}{l}\text { ENSXETG00000 } \\
009683\end{array}$ & tpbgl & 3.54 & $1.79 \mathrm{E}-02$ \\
\hline $\begin{array}{l}\text { ENSXETG00000 } \\
026804\end{array}$ & ptprr & 3.52 & $5.27 \mathrm{E}-03$ \\
\hline $\begin{array}{l}\text { ENSXETG00000 } \\
007501\end{array}$ & fscn1 & 3.51 & 2.46E-09 \\
\hline $\begin{array}{l}\text { ENSXETG00000 } \\
021591\end{array}$ & mcam & 3.51 & 3.00E-03 \\
\hline $\begin{array}{l}\text { ENSXETG00000 } \\
011743\end{array}$ & $h t r 1 b$ & 3.50 & 2.03E-02 \\
\hline $\begin{array}{l}\text { ENSXETG00000 } \\
007838\end{array}$ & adamts7 & 3.49 & $1.50 \mathrm{E}-02$ \\
\hline $\begin{array}{l}\text { ENSXETG00000 } \\
005730\end{array}$ & & 3.49 & $1.79 \mathrm{E}-02$ \\
\hline $\begin{array}{l}\text { ENSXETG00000 } \\
025181\end{array}$ & hoxc3 & 3.48 & $2.46 \mathrm{E}-03$ \\
\hline $\begin{array}{l}\text { ENSXETG00000 } \\
011600\end{array}$ & crmp1 & 3.47 & $1.26 \mathrm{E}-03$ \\
\hline $\begin{array}{l}\text { ENSXETG00000 } \\
019220\end{array}$ & bmpr1b & 3.47 & $1.81 \mathrm{E}-03$ \\
\hline $\begin{array}{l}\text { ENSXETG00000 } \\
010128\end{array}$ & nefm & 3.46 & 3.30E-09 \\
\hline $\begin{array}{l}\text { ENSXETG00000 } \\
023484\end{array}$ & hoxc4 & 3.44 & 9.95E-07 \\
\hline $\begin{array}{l}\text { ENSXETG00000 } \\
018406\end{array}$ & slc24a5 & 3.44 & 7.17E-05 \\
\hline $\begin{array}{l}\text { ENSXETG00000 } \\
019915\end{array}$ & nes & 3.41 & $2.22 \mathrm{E}-02$ \\
\hline $\begin{array}{l}\text { ENSXETG00000 } \\
015623\end{array}$ & cdh19 & 3.41 & 2.66E-02 \\
\hline $\begin{array}{l}\text { ENSXETG00000 } \\
013268\end{array}$ & olfm3 & 3.41 & $2.45 \mathrm{E}-03$ \\
\hline $\begin{array}{l}\text { ENSXETG00000 } \\
004054\end{array}$ & piezo2 & 3.40 & 4.02E-05 \\
\hline $\begin{array}{l}\text { ENSXETG00000 } \\
008490\end{array}$ & kcna1 & 3.39 & 1.90E-02 \\
\hline $\begin{array}{l}\text { ENSXETG00000 } \\
012905\end{array}$ & tyrp1 & 3.39 & $1.26 \mathrm{E}-03$ \\
\hline $\begin{array}{l}\text { ENSXETG00000 } \\
030791\end{array}$ & mmrn2 & 3.38 & 1.44E-02 \\
\hline $\begin{array}{l}\text { ENSXETG00000 } \\
004192\end{array}$ & kcnk18 & 3.36 & 6.66E-03 \\
\hline $\begin{array}{l}\text { ENSXETG00000 } \\
023916\end{array}$ & clstn3 & 3.36 & 8.71E-05 \\
\hline $\begin{array}{l}\text { ENSXETG00000 } \\
019927\end{array}$ & crabp2 & 3.35 & 1.18E-02 \\
\hline $\begin{array}{l}\text { ENSXETG00000 } \\
013830\end{array}$ & plcd4 & 3.33 & 4.88E-03 \\
\hline $\begin{array}{l}\text { ENSXETG00000 } \\
008612\end{array}$ & slc6a8 & 3.33 & $6.09 \mathrm{E}-03$ \\
\hline $\begin{array}{l}\text { ENSXETG00000 } \\
003449\end{array}$ & kcnt1 & 3.31 & 1.25E-02 \\
\hline $\begin{array}{l}\text { ENSXETG00000 } \\
018094\end{array}$ & $\operatorname{scg} 5$ & 3.30 & $3.28 \mathrm{E}-04$ \\
\hline $\begin{array}{l}\text { ENSXETG00000 } \\
001801\end{array}$ & hoxd1 & 3.30 & $5.01 \mathrm{E}-03$ \\
\hline
\end{tabular}




\begin{tabular}{|c|c|c|c|}
\hline $\begin{array}{l}\text { ENSXETG00000 } \\
003372\end{array}$ & ppp1r9b & 3.30 & 3.84E-03 \\
\hline $\begin{array}{l}\text { ENSXETG00000 } \\
017530\end{array}$ & stmn4 & 3.29 & $1.98 \mathrm{E}-02$ \\
\hline $\begin{array}{l}\text { ENSXETG00000 } \\
017708\end{array}$ & foxb1 & 3.29 & 3.35E-02 \\
\hline $\begin{array}{l}\text { ENSXETG00000 } \\
021987\end{array}$ & hoxb6 & 3.28 & 1.70E-02 \\
\hline $\begin{array}{l}\text { ENSXETG00000 } \\
011726\end{array}$ & ntrk2 & 3.27 & 7.97E-04 \\
\hline $\begin{array}{l}\text { ENSXETG00000 } \\
007556\end{array}$ & cd44 & 3.25 & 2.30E-02 \\
\hline $\begin{array}{l}\text { ENSXETG00000 } \\
006011\end{array}$ & slc45a1 & 3.21 & $6.83 \mathrm{E}-03$ \\
\hline $\begin{array}{l}\text { ENSXETG00000 } \\
022371\end{array}$ & $\begin{array}{l}\text { xb-gene- } \\
5730963\end{array}$ & 3.20 & $5.42 \mathrm{E}-03$ \\
\hline $\begin{array}{l}\text { ENSXETG00000 } \\
034183\end{array}$ & mapk8ір3 & 3.20 & 8.96E-04 \\
\hline $\begin{array}{l}\text { ENSXETG00000 } \\
021035\end{array}$ & pdgfra & 3.19 & 1.17E-02 \\
\hline $\begin{array}{l}\text { ENSXETG00000 } \\
014391\end{array}$ & kcnc1 & 3.19 & 4.71E-02 \\
\hline $\begin{array}{l}\text { ENSXETG00000 } \\
009066\end{array}$ & kcnab3 & 3.18 & 1.67E-02 \\
\hline $\begin{array}{l}\text { ENSXETG00000 } \\
027289\end{array}$ & slmo1 & 3.18 & 4.39E-02 \\
\hline $\begin{array}{l}\text { ENSXETG00000 } \\
018862\end{array}$ & $d c l k 1$ & 3.16 & $2.42 \mathrm{E}-02$ \\
\hline $\begin{array}{l}\text { ENSXETG00000 } \\
027719\end{array}$ & tfap2e & 3.16 & $7.86 \mathrm{E}-08$ \\
\hline $\begin{array}{l}\text { ENSXETG00000 } \\
010491\end{array}$ & pcbp2 & 3.16 & $6.55 \mathrm{E}-05$ \\
\hline $\begin{array}{l}\text { ENSXETG00000 } \\
016492\end{array}$ & gng2 & 3.16 & $5.44 \mathrm{E}-03$ \\
\hline $\begin{array}{l}\text { ENSXETG00000 } \\
005309\end{array}$ & Irfn1 & 3.14 & $2.92 \mathrm{E}-02$ \\
\hline $\begin{array}{l}\text { ENSXETG00000 } \\
033812\end{array}$ & tmem116 & 3.13 & 2.43E-02 \\
\hline $\begin{array}{l}\text { ENSXETG00000 } \\
022020\end{array}$ & fam198a & 3.12 & $1.26 \mathrm{E}-02$ \\
\hline $\begin{array}{l}\text { ENSXETG00000 } \\
023385\end{array}$ & fst & 3.12 & 4.15E-02 \\
\hline $\begin{array}{l}\text { ENSXETG00000 } \\
005841\end{array}$ & $n h l h 1$ & 3.12 & $4.20 \mathrm{E}-02$ \\
\hline $\begin{array}{l}\text { ENSXETG00000 } \\
031503\end{array}$ & grik5 & 3.12 & 4.71E-02 \\
\hline $\begin{array}{l}\text { ENSXETG00000 } \\
000442\end{array}$ & optc & 3.11 & $3.68 \mathrm{E}-04$ \\
\hline $\begin{array}{l}\text { ENSXETG00000 } \\
012138\end{array}$ & kif1a & 3.10 & 4.41E-03 \\
\hline $\begin{array}{l}\text { ENSXETG00000 } \\
006647\end{array}$ & $i g d c c 4$ & 3.09 & 4.66E-02 \\
\hline $\begin{array}{l}\text { ENSXETG00000 } \\
005585\end{array}$ & cacnala & 3.08 & 5.32E-03 \\
\hline $\begin{array}{l}\text { ENSXETG00000 } \\
000314\end{array}$ & nr5a2 & 3.07 & 4.41E-03 \\
\hline $\begin{array}{l}\text { ENSXETG00000 } \\
023470\end{array}$ & hoxc12 & 3.06 & 4.71E-02 \\
\hline $\begin{array}{l}\text { ENSXETG00000 } \\
013424\end{array}$ & mfap2 & 3.06 & 7.38E-03 \\
\hline $\begin{array}{l}\text { ENSXETG00000 } \\
023751\end{array}$ & kiaa1274 & 3.05 & $5.83 \mathrm{E}-04$ \\
\hline $\begin{array}{l}\text { ENSXETG00000 } \\
022299\end{array}$ & kal1 & 3.03 & $9.26 \mathrm{E}-03$ \\
\hline $\begin{array}{l}\text { ENSXETG00000 } \\
004290\end{array}$ & ccdc165 & 3.02 & 3.68E-04 \\
\hline $\begin{array}{l}\text { ENSXETG00000 } \\
010095\end{array}$ & nrp2 & 3.01 & 4.41E-03 \\
\hline $\begin{array}{l}\text { ENSXETG00000 } \\
009083\end{array}$ & hes7.2 & 2.99 & 4.29E-02 \\
\hline $\begin{array}{l}\text { ENSXETG00000 } \\
015532\end{array}$ & $f b x \mid 16$ & 2.97 & 3.47E-03 \\
\hline $\begin{array}{l}\text { ENSXETG00000 } \\
023880\end{array}$ & rgmb & 2.95 & $9.80 \mathrm{E}-04$ \\
\hline
\end{tabular}




\begin{tabular}{|c|c|c|c|}
\hline $\begin{array}{l}\text { ENSXETG00000 } \\
020755\end{array}$ & $\operatorname{scg} 3$ & 2.95 & 4.91E-02 \\
\hline $\begin{array}{l}\text { ENSXETG00000 } \\
030958\end{array}$ & kcnh5 & 2.95 & $1.26 \mathrm{E}-02$ \\
\hline $\begin{array}{l}\text { ENSXETG00000 } \\
003986\end{array}$ & Imo2 & 2.95 & 3.12E-02 \\
\hline $\begin{array}{l}\text { ENSXETG00000 } \\
021660\end{array}$ & scarf2 & 2.93 & $2.77 \mathrm{E}-02$ \\
\hline $\begin{array}{l}\text { ENSXETG00000 } \\
021341\end{array}$ & krt16 & 2.93 & 1.75E-07 \\
\hline $\begin{array}{l}\text { ENSXETG00000 } \\
006199\end{array}$ & mapk10 & 2.93 & $9.02 \mathrm{E}-04$ \\
\hline $\begin{array}{l}\text { ENSXETG00000 } \\
012355\end{array}$ & dach1 & 2.92 & $4.71 \mathrm{E}-02$ \\
\hline $\begin{array}{l}\text { ENSXETG00000 } \\
001772\end{array}$ & gdap1/1 & 2.92 & $3.42 \mathrm{E}-04$ \\
\hline $\begin{array}{l}\text { ENSXETG00000 } \\
015485\end{array}$ & mycn & 2.92 & 3.31E-04 \\
\hline $\begin{array}{l}\text { ENSXETG00000 } \\
020864\end{array}$ & ppp2r2b & 2.91 & 3.87E-04 \\
\hline $\begin{array}{l}\text { ENSXETG00000 } \\
022243\end{array}$ & cadps & 2.89 & $2.12 \mathrm{E}-03$ \\
\hline $\begin{array}{l}\text { ENSXETG00000 } \\
033848\end{array}$ & & 2.88 & 1.34E-04 \\
\hline $\begin{array}{l}\text { ENSXETG00000 } \\
001405\end{array}$ & & 2.87 & $4.71 \mathrm{E}-02$ \\
\hline $\begin{array}{l}\text { ENSXETG00000 } \\
031488\end{array}$ & pcdh19 & 2.87 & 1.79E-02 \\
\hline $\begin{array}{l}\text { ENSXETG00000 } \\
003707\end{array}$ & apc2 & 2.85 & $2.77 \mathrm{E}-03$ \\
\hline $\begin{array}{l}\text { ENSXETG00000 } \\
030305\end{array}$ & amph & 2.85 & 3.50E-02 \\
\hline $\begin{array}{l}\text { ENSXETG00000 } \\
014034\end{array}$ & lix1l & 2.81 & $5.84 \mathrm{E}-03$ \\
\hline $\begin{array}{l}\text { ENSXETG00000 } \\
003954\end{array}$ & ptgds & 2.79 & $1.25 \mathrm{E}-02$ \\
\hline $\begin{array}{l}\text { ENSXETG00000 } \\
007462\end{array}$ & vim & 2.77 & $1.92 \mathrm{E}-06$ \\
\hline $\begin{array}{l}\text { ENSXETG00000 } \\
008109\end{array}$ & $x k r 4$ & 2.77 & 5.57E-03 \\
\hline $\begin{array}{l}\text { ENSXETG00000 } \\
018024\end{array}$ & c6orf145 & 2.74 & 7.93E-03 \\
\hline $\begin{array}{l}\text { ENSXETG00000 } \\
032893\end{array}$ & plekhg4b & 2.74 & $2.56 \mathrm{E}-02$ \\
\hline $\begin{array}{l}\text { ENSXETG00000 } \\
027686\end{array}$ & serpinf1 & 2.72 & 3.81E-04 \\
\hline $\begin{array}{l}\text { ENSXETG00000 } \\
020206\end{array}$ & timp2 & 2.72 & $3.28 \mathrm{E}-02$ \\
\hline $\begin{array}{l}\text { ENSXETG00000 } \\
010662\end{array}$ & $a p 3 b 2$ & 2.71 & 4.76E-04 \\
\hline $\begin{array}{l}\text { ENSXETG00000 } \\
002780\end{array}$ & clip3 & 2.70 & $2.06 \mathrm{E}-03$ \\
\hline $\begin{array}{l}\text { ENSXETG00000 } \\
007241\end{array}$ & $d b n 1$ & 2.69 & $1.20 \mathrm{E}-05$ \\
\hline $\begin{array}{l}\text { ENSXETG00000 } \\
000735\end{array}$ & srpk2 & 2.69 & $1.04 \mathrm{E}-02$ \\
\hline $\begin{array}{l}\text { ENSXETG00000 } \\
022354\end{array}$ & nos1 & 2.69 & 1.12E-03 \\
\hline $\begin{array}{l}\text { ENSXETG00000 } \\
013597\end{array}$ & unc13a & 2.63 & 2.92E-02 \\
\hline $\begin{array}{l}\text { ENSXETG00000 } \\
003735\end{array}$ & pcdh8I & 2.63 & 3.64E-02 \\
\hline $\begin{array}{l}\text { ENSXETG00000 } \\
020455\end{array}$ & krt18 & 2.63 & $5.35 \mathrm{E}-10$ \\
\hline $\begin{array}{l}\text { ENSXETG00000 } \\
002894\end{array}$ & kcnab2 & 2.62 & 2.43E-02 \\
\hline $\begin{array}{l}\text { ENSXETG00000 } \\
011446\end{array}$ & plxna4 & 2.60 & 3.41E-02 \\
\hline $\begin{array}{l}\text { ENSXETG00000 } \\
032158\end{array}$ & wasf3 & 2.60 & 2.92E-02 \\
\hline $\begin{array}{l}\text { ENSXETG00000 } \\
009658\end{array}$ & $m r z 09$ & 2.60 & $6.66 \mathrm{E}-03$ \\
\hline $\begin{array}{l}\text { ENSXETG00000 } \\
021674\end{array}$ & tfap2b & 2.57 & 1.17E-04 \\
\hline
\end{tabular}




\begin{tabular}{|c|c|c|c|}
\hline $\begin{array}{l}\text { ENSXETG00000 } \\
022636\end{array}$ & prss35 & 2.57 & 7.37E-05 \\
\hline $\begin{array}{l}\text { ENSXETG00000 } \\
003570\end{array}$ & cxcl12 & 2.55 & 7.71E-03 \\
\hline $\begin{array}{l}\text { ENSXETG00000 } \\
015769\end{array}$ & nrcam & 2.53 & 1.70E-02 \\
\hline $\begin{array}{l}\text { ENSXETG00000 } \\
002702\end{array}$ & mmp24 & 2.52 & $1.78 \mathrm{E}-02$ \\
\hline $\begin{array}{l}\text { ENSXETG00000 } \\
023036\end{array}$ & kcnq3 & 2.51 & 4.33E-02 \\
\hline $\begin{array}{l}\text { ENSXETG00000 } \\
018853\end{array}$ & $r a b 3 c$ & 2.49 & $2.56 \mathrm{E}-02$ \\
\hline $\begin{array}{l}\text { ENSXETG00000 } \\
012785\end{array}$ & $b s n$ & 2.46 & $2.90 \mathrm{E}-03$ \\
\hline $\begin{array}{l}\text { ENSXETG00000 } \\
001650\end{array}$ & ppp2r5b & 2.44 & $1.80 \mathrm{E}-02$ \\
\hline $\begin{array}{l}\text { ENSXETG00000 } \\
017780\end{array}$ & fez1 & 2.43 & $1.79 \mathrm{E}-02$ \\
\hline $\begin{array}{l}\text { ENSXETG00000 } \\
003374\end{array}$ & col1a1 & 2.42 & $1.29 \mathrm{E}-08$ \\
\hline $\begin{array}{l}\text { ENSXETG00000 } \\
011604\end{array}$ & ppp2r2c & 2.42 & 4.59E-02 \\
\hline $\begin{array}{l}\text { ENSXETG00000 } \\
012934\end{array}$ & $b g n$ & 2.41 & 8.09E-05 \\
\hline $\begin{array}{l}\text { ENSXETG00000 } \\
031380\end{array}$ & gng3 & 2.40 & 4.49E-03 \\
\hline $\begin{array}{l}\text { ENSXETG00000 } \\
002263\end{array}$ & $\operatorname{ttc} 28$ & 2.39 & 2.03E-02 \\
\hline $\begin{array}{l}\text { ENSXETG00000 } \\
016983\end{array}$ & $r \operatorname{tn} 1$ & 2.38 & 4.83E-03 \\
\hline $\begin{array}{l}\text { ENSXETG00000 } \\
020369\end{array}$ & sgip1 & 2.37 & $2.42 \mathrm{E}-02$ \\
\hline $\begin{array}{l}\text { ENSXETG00000 } \\
011375\end{array}$ & mmp25 & 2.36 & 8.46E-04 \\
\hline $\begin{array}{l}\text { ENSXETG00000 } \\
030341\end{array}$ & rab3il1 & 2.35 & $2.86 \mathrm{E}-02$ \\
\hline $\begin{array}{l}\text { ENSXETG00000 } \\
029995\end{array}$ & sptbn1 & 2.35 & 4.04E-06 \\
\hline $\begin{array}{l}\text { ENSXETG00000 } \\
011332\end{array}$ & vwde & 2.32 & 4.71E-02 \\
\hline $\begin{array}{l}\text { ENSXETG00000 } \\
000720\end{array}$ & hoxa5 & 2.32 & 4.22E-03 \\
\hline $\begin{array}{l}\text { ENSXETG00000 } \\
015555\end{array}$ & ank2 & 2.31 & $6.66 \mathrm{E}-03$ \\
\hline $\begin{array}{l}\text { ENSXETG00000 } \\
009096\end{array}$ & vamp2 & 2.31 & 4.71E-02 \\
\hline $\begin{array}{l}\text { ENSXETG00000 } \\
006707\end{array}$ & b4galnt4 & 2.31 & $1.27 \mathrm{E}-02$ \\
\hline $\begin{array}{l}\text { ENSXETG00000 } \\
011788\end{array}$ & $c d \times 2$ & 2.30 & $2.20 \mathrm{E}-03$ \\
\hline $\begin{array}{l}\text { ENSXETG00000 } \\
017443\end{array}$ & glt25d2 & 2.26 & $2.13 \mathrm{E}-02$ \\
\hline $\begin{array}{l}\text { ENSXETG00000 } \\
011665\end{array}$ & ncam1 & 2.24 & 6.66E-03 \\
\hline $\begin{array}{l}\text { ENSXETG00000 } \\
031133\end{array}$ & sema3e & 2.24 & $1.79 \mathrm{E}-02$ \\
\hline $\begin{array}{l}\text { ENSXETG00000 } \\
010393\end{array}$ & & 2.23 & $2.08 \mathrm{E}-03$ \\
\hline $\begin{array}{l}\text { ENSXETG00000 } \\
034301\end{array}$ & & 2.21 & $2.25 \mathrm{E}-04$ \\
\hline $\begin{array}{l}\text { ENSXETG00000 } \\
004486\end{array}$ & kif5c & 2.21 & 3.76E-02 \\
\hline $\begin{array}{l}\text { ENSXETG00000 } \\
017239\end{array}$ & & 2.20 & $2.76 \mathrm{E}-03$ \\
\hline $\begin{array}{l}\text { ENSXETG00000 } \\
011188\end{array}$ & prph & 2.19 & 4.41E-03 \\
\hline $\begin{array}{l}\text { ENSXETG00000 } \\
007431\end{array}$ & adam22 & 2.19 & $2.49 \mathrm{E}-03$ \\
\hline $\begin{array}{l}\text { ENSXETG00000 } \\
004589\end{array}$ & $c d \times 4$ & 2.18 & 4.38E-03 \\
\hline $\begin{array}{l}\text { ENSXETG00000 } \\
022798\end{array}$ & sostdc1 & 2.16 & 3.47E-02 \\
\hline $\begin{array}{l}\text { ENSXETG00000 } \\
026058\end{array}$ & cnfn.1 & 2.13 & 1.07E-02 \\
\hline
\end{tabular}




\begin{tabular}{|c|c|c|c|}
\hline $\begin{array}{l}\text { ENSXETG00000 } \\
020346\end{array}$ & gabbr1 & 2.09 & $3.51 \mathrm{E}-03$ \\
\hline $\begin{array}{l}\text { ENSXETG00000 } \\
032203\end{array}$ & map1a & 2.08 & 3.10E-02 \\
\hline $\begin{array}{l}\text { ENSXETG00000 } \\
002308\end{array}$ & rims1 & 2.08 & $4.63 \mathrm{E}-02$ \\
\hline $\begin{array}{l}\text { ENSXETG00000 } \\
007363\end{array}$ & rundc3a & 2.06 & $9.90 \mathrm{E}-04$ \\
\hline $\begin{array}{l}\text { ENSXETG00000 } \\
018184\end{array}$ & сур26a1 & 2.06 & $9.26 \mathrm{E}-03$ \\
\hline $\begin{array}{l}\text { ENSXETG00000 } \\
025407\end{array}$ & sp5 & 2.06 & $2.08 \mathrm{E}-02$ \\
\hline $\begin{array}{l}\text { ENSXETG00000 } \\
030115\end{array}$ & sp8 & 2.05 & 1.46E-08 \\
\hline $\begin{array}{l}\text { ENSXETG00000 } \\
012198\end{array}$ & nid2 & 2.03 & 1.72E-06 \\
\hline $\begin{array}{l}\text { ENSXETG00000 } \\
015806\end{array}$ & hecw1 & 2.03 & 3.37E-02 \\
\hline $\begin{array}{l}\text { ENSXETG00000 } \\
027090\end{array}$ & $\begin{array}{l}\text { xb-gene- } \\
5768884\end{array}$ & 2.01 & $6.07 \mathrm{E}-04$ \\
\hline $\begin{array}{l}\text { ENSXETG00000 } \\
013742\end{array}$ & ndrg3 & 2.00 & $6.66 \mathrm{E}-03$ \\
\hline $\begin{array}{l}\text { ENSXETG00000 } \\
005949\end{array}$ & mst1 & 1.99 & 3.48E-02 \\
\hline $\begin{array}{l}\text { ENSXETG00000 } \\
018032\end{array}$ & $t u b b 2 b$ & 1.98 & 4.85E-04 \\
\hline $\begin{array}{l}\text { ENSXETG00000 } \\
008971\end{array}$ & ywhag & 1.96 & 8.73E-04 \\
\hline $\begin{array}{l}\text { ENSXETG00000 } \\
021967\end{array}$ & hoxb3 & 1.95 & 4.18E-02 \\
\hline $\begin{array}{l}\text { ENSXETG00000 } \\
016758\end{array}$ & $\begin{array}{l}x b \text {-gene- } \\
1002559\end{array}$ & 1.95 & $2.24 \mathrm{E}-06$ \\
\hline $\begin{array}{l}\text { ENSXETG00000 } \\
020280\end{array}$ & fam171a1 & 1.94 & 1.97E-04 \\
\hline $\begin{array}{l}\text { ENSXETG00000 } \\
024006\end{array}$ & snai2 & 1.92 & $2.69 \mathrm{E}-03$ \\
\hline $\begin{array}{l}\text { ENSXETG00000 } \\
031472\end{array}$ & tenm2 & 1.91 & $1.85 \mathrm{E}-05$ \\
\hline $\begin{array}{l}\text { ENSXETG00000 } \\
031338\end{array}$ & cntn4 & 1.91 & $2.89 \mathrm{E}-02$ \\
\hline $\begin{array}{l}\text { ENSXETG00000 } \\
014880\end{array}$ & $\begin{array}{l}\text { xb-gene- } \\
5936529\end{array}$ & 1.89 & $2.38 \mathrm{E}-02$ \\
\hline $\begin{array}{l}\text { ENSXETG00000 } \\
017270\end{array}$ & $c 3$ & 1.88 & $1.04 \mathrm{E}-02$ \\
\hline $\begin{array}{l}\text { ENSXETG00000 } \\
010655\end{array}$ & col2a1 & 1.88 & 4.18E-06 \\
\hline $\begin{array}{l}\text { ENSXETG00000 } \\
017823\end{array}$ & plxnb1 & 1.88 & 4.83E-03 \\
\hline $\begin{array}{l}\text { ENSXETG00000 } \\
016109\end{array}$ & gatm & 1.86 & $6.29 \mathrm{E}-07$ \\
\hline $\begin{array}{l}\text { ENSXETG00000 } \\
007614\end{array}$ & epha4 & 1.86 & $5.55 \mathrm{E}-03$ \\
\hline $\begin{array}{l}\text { ENSXETG00000 } \\
013228\end{array}$ & egflam & 1.86 & 2.99E-03 \\
\hline $\begin{array}{l}\text { ENSXETG00000 } \\
019579\end{array}$ & pmp22 & 1.80 & $1.04 \mathrm{E}-02$ \\
\hline $\begin{array}{l}\text { ENSXETG00000 } \\
009499\end{array}$ & slc39a5 & 1.80 & $8.81 \mathrm{E}-04$ \\
\hline $\begin{array}{l}\text { ENSXETG00000 } \\
027751\end{array}$ & marcks & 1.79 & $1.71 \mathrm{E}-03$ \\
\hline $\begin{array}{l}\text { ENSXETG00000 } \\
005960\end{array}$ & c3orf54 & 1.79 & $9.28 \mathrm{E}-04$ \\
\hline $\begin{array}{l}\text { ENSXETG00000 } \\
023647\end{array}$ & rufy3 & 1.78 & $1.53 \mathrm{E}-03$ \\
\hline $\begin{array}{l}\text { ENSXETG00000 } \\
008706\end{array}$ & flrt3 & 1.71 & 1.18E-02 \\
\hline $\begin{array}{l}\text { ENSXETG00000 } \\
010898\end{array}$ & $f n 1$ & 1.66 & 8.57E-03 \\
\hline $\begin{array}{l}\text { ENSXETG00000 } \\
010192\end{array}$ & pde4dip & 1.66 & 2.43E-02 \\
\hline $\begin{array}{l}\text { ENSXETG00000 } \\
002710\end{array}$ & rcor2 & 1.65 & 4.71E-02 \\
\hline $\begin{array}{l}\text { ENSXETG00000 } \\
005775\end{array}$ & gng13 & 1.65 & 4.73E-03 \\
\hline
\end{tabular}




\begin{tabular}{|c|c|c|c|}
\hline $\begin{array}{l}\text { ENSXETG00000 } \\
004959\end{array}$ & epb41/3 & 1.63 & 2.13E-02 \\
\hline $\begin{array}{l}\text { ENSXETG00000 } \\
000172\end{array}$ & maf & 1.61 & $3.27 \mathrm{E}-02$ \\
\hline $\begin{array}{l}\text { ENSXETG00000 } \\
021398\end{array}$ & $d g k i$ & 1.61 & $4.26 \mathrm{E}-03$ \\
\hline $\begin{array}{l}\text { ENSXETG00000 } \\
008088\end{array}$ & & 1.58 & $4.81 \mathrm{E}-02$ \\
\hline $\begin{array}{l}\text { ENSXETG00000 } \\
002597\end{array}$ & Ipar1 & 1.57 & $4.71 \mathrm{E}-02$ \\
\hline $\begin{array}{l}\text { ENSXETG00000 } \\
007569\end{array}$ & hapln3 & 1.57 & $9.12 \mathrm{E}-03$ \\
\hline $\begin{array}{l}\text { ENSXETG00000 } \\
011877\end{array}$ & $c d 74$ & 1.53 & $1.60 \mathrm{E}-02$ \\
\hline $\begin{array}{l}\text { ENSXETG00000 } \\
020022\end{array}$ & gnao1 & 1.53 & 1.06E-02 \\
\hline $\begin{array}{l}\text { ENSXETG00000 } \\
031404\end{array}$ & draxin & 1.49 & $1.77 \mathrm{E}-03$ \\
\hline $\begin{array}{l}\text { ENSXETG00000 } \\
018597\end{array}$ & $a l d h 3 b 1$ & 1.47 & $6.85 \mathrm{E}-04$ \\
\hline $\begin{array}{l}\text { ENSXETG00000 } \\
007165\end{array}$ & myo10.2 & 1.47 & 4.55E-02 \\
\hline $\begin{array}{l}\text { ENSXETG00000 } \\
024138\end{array}$ & rgma & 1.46 & $2.76 \mathrm{E}-03$ \\
\hline $\begin{array}{l}\text { ENSXETG00000 } \\
011757\end{array}$ & col5a3 & 1.46 & 1.70E-02 \\
\hline $\begin{array}{l}\text { ENSXETG00000 } \\
011615\end{array}$ & fam171a2 & 1.44 & 2.39E-02 \\
\hline $\begin{array}{l}\text { ENSXETG00000 } \\
013891\end{array}$ & kcnj13 & 1.39 & 3.95E-02 \\
\hline $\begin{array}{l}\text { ENSXETG00000 } \\
012216\end{array}$ & msi1 & 1.39 & 1.36E-02 \\
\hline $\begin{array}{l}\text { ENSXETG00000 } \\
025032\end{array}$ & mmp15 & 1.36 & $2.21 \mathrm{E}-02$ \\
\hline $\begin{array}{l}\text { ENSXETG00000 } \\
013633\end{array}$ & $r a b 30$ & 1.36 & 1.92E-02 \\
\hline $\begin{array}{l}\text { ENSXETG00000 } \\
020090\end{array}$ & pfn2 & 1.34 & 1.74E-02 \\
\hline $\begin{array}{l}\text { ENSXETG00000 } \\
003486\end{array}$ & st3gal3 & 1.31 & 4.10E-02 \\
\hline $\begin{array}{l}\text { ENSXETG00000 } \\
010561\end{array}$ & $d m d$ & 1.30 & $1.40 \mathrm{E}-02$ \\
\hline $\begin{array}{l}\text { ENSXETG00000 } \\
018626\end{array}$ & $x k$ & 1.30 & 4.71E-02 \\
\hline $\begin{array}{l}\text { ENSXETG00000 } \\
011231\end{array}$ & $\mathrm{cmtm} 3$ & 1.30 & 2.96E-02 \\
\hline $\begin{array}{l}\text { ENSXETG00000 } \\
004468\end{array}$ & kiaa0182 & 1.28 & 2.33E-03 \\
\hline $\begin{array}{l}\text { ENSXETG00000 } \\
034199\end{array}$ & prkg1 & 1.26 & 8.08E-04 \\
\hline $\begin{array}{l}\text { ENSXETG00000 } \\
001502\end{array}$ & cyfip2 & 1.21 & $1.65 \mathrm{E}-02$ \\
\hline $\begin{array}{l}\text { ENSXETG00000 } \\
018465\end{array}$ & rell1 & 1.21 & 3.90E-02 \\
\hline $\begin{array}{l}\text { ENSXETG00000 } \\
017308\end{array}$ & dlgap4 & 1.18 & $5.73 \mathrm{E}-03$ \\
\hline $\begin{array}{l}\text { ENSXETG00000 } \\
027945\end{array}$ & slc30a8 & 1.09 & $2.24 \mathrm{E}-02$ \\
\hline $\begin{array}{l}\text { ENSXETG00000 } \\
011833\end{array}$ & ptprd & 1.05 & 2.03E-02 \\
\hline $\begin{array}{l}\text { ENSXETG00000 } \\
023814\end{array}$ & angpt4 & 1.05 & 3.06E-03 \\
\hline $\begin{array}{l}\text { ENSXETG00000 } \\
024861\end{array}$ & Ioxl1 & 1.05 & $3.48 \mathrm{E}-02$ \\
\hline $\begin{array}{l}\text { ENSXETG00000 } \\
018462\end{array}$ & $n k d 1$ & 1.03 & 7.00E-03 \\
\hline $\begin{array}{l}\text { ENSXETG00000 } \\
014931\end{array}$ & pnkd & 1.00 & $2.92 \mathrm{E}-02$ \\
\hline
\end{tabular}




\subsection{GO analysis of candidate genes}

Gene Ontology (GO) analysis was performed using DAVID (http://david.abcc.ncifcrf.gov) as previously described (Dennis et al., 2003). Analyzed were genes which were up and downregulated at stage 14 and 27, respectively.

\subsubsection{GO analysis of candidate genes upregulated at stage 14}

Given is the biological process, the number of genes and the P-value. Biological processes were sorted according to the P-value from lowest to highest.

Table 6.3 Summary of GO analysis of genes upregulated at stage 14

\begin{tabular}{|c|c|c|}
\hline Biological Processes & Count & P-value \\
\hline GO:0007389 pattern specification process & 33 & $5.20 \mathrm{E}-17$ \\
\hline GO:0003002 regionalization & 28 & $6.70 \mathrm{E}-16$ \\
\hline GO:0001501 skeletal system development & 34 & $1.55 \mathrm{E}-15$ \\
\hline GO:0009952 anterior/posterior pattern formation & 24 & $1.71 \mathrm{E}-15$ \\
\hline GO:0009792 embryonic development ending in birth or egg hatching & 30 & 7.64E-12 \\
\hline GO:0043009 chordate embryonic development & 29 & 3.36E-11 \\
\hline GO:0048568 embryonic organ development & 20 & $6.65 \mathrm{E}-10$ \\
\hline GO:0048598 embryonic morphogenesis & 26 & 8.97E-10 \\
\hline GO:0048706 embryonic skeletal system development & 13 & $2.10 \mathrm{E}-08$ \\
\hline GO:0048705 skeletal system morphogenesis & 14 & 1.98E-07 \\
\hline GO:0016055 Wnt receptor signaling pathway & 15 & $2.29 \mathrm{E}-07$ \\
\hline GO:0030182 neuron differentiation & 27 & $2.72 \mathrm{E}-07$ \\
\hline GO:0006355 regulation of transcription, DNA-dependent & 61 & 4.31E-06 \\
\hline GO:0048562 embryonic organ morphogenesis & 13 & 8.57E-06 \\
\hline GO:0051252 regulation of RNA metabolic process & 61 & 8.75E-06 \\
\hline GO:0045165 cell fate commitment & 13 & 1.35E-05 \\
\hline GO:0035107 appendage morphogenesis & 11 & $1.82 \mathrm{E}-05$ \\
\hline GO:0035108 limb morphogenesis & 11 & $1.82 \mathrm{E}-05$ \\
\hline GO:0035295 tube development & 16 & 2.02E-05 \\
\hline GO:0002009 morphogenesis of an epithelium & 11 & 2.17E-05 \\
\hline GO:0048736 appendage development & 11 & $2.57 \mathrm{E}-05$ \\
\hline GO:0060173 limb development & 11 & $2.57 \mathrm{E}-05$ \\
\hline GO:0042127 regulation of cell proliferation & 33 & 3.83E-05 \\
\hline GO:0006357 regulation of transcription from RNA polymerase II promoter & 31 & $5.19 \mathrm{E}-05$ \\
\hline GO:0045449 regulation of transcription & 76 & $6.73 E-05$ \\
\hline GO:0001568 blood vessel development & 16 & 6.99E-05 \\
\hline
\end{tabular}




\begin{tabular}{|c|c|c|}
\hline GO:0001944 vasculature development & 16 & $9.18 \mathrm{E}-05$ \\
\hline GO:0048704 embryonic skeletal system morphogenesis & 8 & $9.68 \mathrm{E}-05$ \\
\hline GO:0006350-transcription & 64 & 1.05E-04 \\
\hline GO:0060429 epithelium development & 15 & 1.13E-04 \\
\hline GO:0031175 neuron projection development & 16 & 1.15E-04 \\
\hline GO:0060348 bone development & 11 & 1.17E-04 \\
\hline GO:0048729 tissue morphogenesis & 13 & 1.69E-04 \\
\hline GO:0030326 embryonic limb morphogenesis & 9 & 2.44E-04 \\
\hline GO:0035113 embryonic appendage morphogenesis & 9 & 2.44E-04 \\
\hline GO:0051960 regulation of nervous system development & 13 & 3.07E-04 \\
\hline GO:0051893 regulation of focal adhesion formation & 4 & $3.54 \mathrm{E}-04$ \\
\hline GO:0070482 response to oxygen levels & 11 & 3.59E-04 \\
\hline GO:0022610 biological adhesion & 28 & $3.78 \mathrm{E}-04$ \\
\hline GO:0007155 cell adhesion & 28 & 3.78E-04 \\
\hline GO:0007223 Wnt receptor signaling pathway, calcium modulating pathway & 5 & $5.90 \mathrm{E}-04$ \\
\hline GO:0031328 positive regulation of cellular biosynthetic process & 27 & $6.12 \mathrm{E}-04$ \\
\hline GO:0030030 cell projection organization & 18 & $6.79 \mathrm{E}-04$ \\
\hline GO:0010557 positive regulation of macromolecule biosynthetic process & 26 & 7.03E-04 \\
\hline GO:0030323 respiratory tube development & 9 & 7.16E-04 \\
\hline GO:0048514 blood vessel morphogenesis & 13 & 7.19E-04 \\
\hline GO:0009891 positive regulation of biosynthetic process & 27 & 7.53E-04 \\
\hline GO:0051094 positive regulation of developmental process & 15 & $8.82 \mathrm{E}-04$ \\
\hline GO:0022604 regulation of cell morphogenesis & 10 & 8.83E-04 \\
\hline GO:0001890 placenta development & 7 & $9.41 \mathrm{E}-04$ \\
\hline GO:0008284 positive regulation of cell proliferation & 19 & $9.51 \mathrm{E}-04$ \\
\hline GO:0045664 regulation of neuron differentiation & 10 & $9.84 \mathrm{E}-04$ \\
\hline GO:0001666 response to hypoxia & 10 & 0.001037845 \\
\hline GO:0050767 regulation of neurogenesis & 11 & 0.001277265 \\
\hline GO:0048146 positive regulation of fibroblast proliferation & 5 & 0.001368572 \\
\hline GO:0045597 positive regulation of cell differentiation & 13 & 0.001454326 \\
\hline GO:0031344 regulation of cell projection organization & 8 & 0.001507788 \\
\hline GO:0001503 ossification & 9 & 0.001564536 \\
\hline GO:0010810 regulation of cell-substrate adhesion & 6 & 0.001727396 \\
\hline GO:0021915 neural tube development & 7 & 0.001813197 \\
\hline GO:0006928 cell motion & 20 & 0.001833765 \\
\hline GO:0060284 regulation of cell development & 12 & 0.00188405 \\
\hline GO:0035270 endocrine system development & 7 & 0.00195489 \\
\hline GO:0035282 segmentation & 6 & 0.002092464 \\
\hline GO:0010975 regulation of neuron projection development & 7 & 0.002104804 \\
\hline GO:0048666 neuron development & 16 & 0.002122653 \\
\hline GO:0001822 kidney development & 8 & 0.002332143 \\
\hline GO:0048812 neuron projection morphogenesis & 12 & 0.002542962 \\
\hline GO:0001708 cell fate specification & 6 & 0.002741333 \\
\hline GO:0051173 positive regulation of nitrogen compound metabolic process & 24 & 0.002765258 \\
\hline Go:0045596 negative regulation of cell differentiation & 12 & 0.002834022 \\
\hline GO:0035239 tube morphogenesis & 9 & 0.002920086 \\
\hline
\end{tabular}




\begin{tabular}{|c|c|c|}
\hline GO:0060485 mesenchyme development & 6 & 0.002986784 \\
\hline GO:0014033 neural crest cell differentiation & 5 & 0.00337608 \\
\hline GO:0014032 neural crest cell development & 5 & 0.00337608 \\
\hline GO:0010769 regulation of cell morphogenesis involved in differentiation & 7 & 0.003409922 \\
\hline GO:0051895 negative regulation of focal adhesion formation & 3 & 0.003461661 \\
\hline GO:0010604 positive regulation of macromolecule metabolic process & 29 & 0.003550878 \\
\hline $\begin{array}{l}\text { GO:0045935 positive regulation of nucleobase, nucleoside, nucleotide and } \\
\text { nucleic acid metabolic process }\end{array}$ & 23 & 0.003923674 \\
\hline GO:0048145 regulation of fibroblast proliferation & 5 & 0.004193864 \\
\hline GO:0050770 regulation of axonogenesis & 6 & 0.00445706 \\
\hline GO:0045893 positive regulation of transcription, DNA-dependent & 19 & 0.004459939 \\
\hline GO:0030155 regulation of cell adhesion & 9 & 0.004634042 \\
\hline $\begin{array}{l}\begin{array}{l}\text { GO:0045944 positive regulation of transcription from RNA polymerase II } \\
\text { promoter }\end{array} \\
\end{array}$ & 16 & 0.00488243 \\
\hline GO:0051254 positive regulation of RNA metabolic process & 19 & 0.00490873 \\
\hline GO:0001655 urogenital system development & 8 & 0.00498123 \\
\hline GO:0006469 negative regulation of protein kinase activity & 7 & 0.006210497 \\
\hline GO:0016477 cell migration & 13 & 0.006593595 \\
\hline GO:0000904 cell morphogenesis involved in differentiation & 12 & 0.007032767 \\
\hline GO:0002011 morphogenesis of an epithelial sheet & 3 & 0.007088879 \\
\hline GO:0060216 definitive hemopoiesis & 3 & 0.007088879 \\
\hline GO:0048858 cell projection morphogenesis & 12 & 0.007244738 \\
\hline GO:0033673 negative regulation of kinase activity & 7 & 0.007306829 \\
\hline GO:0001570 vasculogenesis & 5 & 0.007423532 \\
\hline GO:0010628 positive regulation of gene expression & 21 & 0.007600185 \\
\hline GO:0010629 negative regulation of gene expression & 19 & 0.007718019 \\
\hline GO:0051130 positive regulation of cellular component organization & 10 & 0.007749873 \\
\hline GO:0035136 forelimb morphogenesis & 4 & 0.007990003 \\
\hline GO:0007507 heart development & 11 & 0.008095522 \\
\hline GO:0000902 cell morphogenesis & 15 & 0.00825573 \\
\hline GO:0045892 negative regulation of transcription, DNA-dependent & 15 & 0.00825573 \\
\hline GO:0043549 regulation of kinase activity & 15 & 0.008437681 \\
\hline GO:0009855 determination of bilateral symmetry & 5 & 0.008783876 \\
\hline GO:0009799 determination of symmetry & 5 & 0.008783876 \\
\hline GO:0060562 epithelial tube morphogenesis & 6 & 0.008824926 \\
\hline GO:0032989 cellular component morphogenesis & 16 & 0.009012378 \\
\hline GO:0009954 proximal/distal pattern formation & 4 & 0.009061354 \\
\hline GO:0051253 negative regulation of RNA metabolic process & 15 & 0.009381825 \\
\hline GO:0043583 ear development & 7 & 0.009434829 \\
\hline GO:0051348 negative regulation of transferase activity & 7 & 0.009908341 \\
\hline GO:0032990 cell part morphogenesis & 12 & 0.009912217 \\
\hline GO:0010720 positive regulation of cell development & 6 & 0.00996161 \\
\hline GO:0042981 regulation of apoptosis & 26 & 0.010494514 \\
\hline GO:0040012 regulation of locomotion & 10 & 0.011167629 \\
\hline GO:0030324 lung development & 7 & 0.01143017 \\
\hline GO:0050772 positive regulation of axonogenesis & 4 & 0.011445024 \\
\hline GO:0045941 positive regulation of transcription & 20 & 0.011537427 \\
\hline
\end{tabular}




\begin{tabular}{|c|c|c|}
\hline GO:0007409 axonogenesis & 10 & 0.011545858 \\
\hline GO:0043067 regulation of programmed cell death & 26 & 0.0118267 \\
\hline GO:0051338 regulation of transferase activity & 15 & 0.011865452 \\
\hline GO:0031346 positive regulation of cell projection organization & 5 & 0.011970439 \\
\hline GO:0010941 regulation of cell death & 26 & 0.01219235 \\
\hline GO:0007423 sensory organ development & 11 & 0.012269742 \\
\hline GO:0001952 regulation of cell-matrix adhesion & 4 & 0.014156404 \\
\hline GO:0016481 negative regulation of transcription & 17 & 0.014505195 \\
\hline GO:0048870 cell motility & 13 & 0.014567628 \\
\hline GO:0051674 localization of cell & 13 & 0.014567628 \\
\hline GO:0001953 negative regulation of cell-matrix adhesion & 3 & 0.01463005 \\
\hline GO:0045859 regulation of protein kinase activity & 14 & 0.01481493 \\
\hline GO:0030334 regulation of cell migration & 9 & 0.015480762 \\
\hline GO:0010811 positive regulation of cell-substrate adhesion & 4 & 0.015636844 \\
\hline GO:0014031 mesenchymal cell development & 5 & 0.015816193 \\
\hline GO:0048762 mesenchymal cell differentiation & 5 & 0.015816193 \\
\hline GO:0030278 regulation of ossification & 6 & 0.016323553 \\
\hline GO:0016337 cell-cell adhesion & 12 & 0.016588266 \\
\hline GO:0060541 respiratory system development & 7 & 0.01697508 \\
\hline GO:0048839 inner ear development & 6 & 0.017164717 \\
\hline GO:0043065 positive regulation of apoptosis & 16 & 0.017541166 \\
\hline GO:0010812 negative regulation of cell-substrate adhesion & 3 & 0.017659224 \\
\hline GO:0043405 regulation of MAP kinase activity & 8 & 0.018111471 \\
\hline GO:0048667 cell morphogenesis involved in neuron differentiation & 10 & 0.01852925 \\
\hline GO:0043068 positive regulation of programmed cell death & 16 & 0.018540644 \\
\hline GO:0044087 regulation of cellular component biogenesis & 8 & 0.018753038 \\
\hline GO:0060606 tube closure & 4 & 0.01884975 \\
\hline GO:0001843 neural tube closure & 4 & 0.01884975 \\
\hline GO:0010942 positive regulation of cell death & 16 & 0.019262565 \\
\hline GO:0010033 response to organic substance & 23 & 0.019360157 \\
\hline GO:0008285 negative regulation of cell proliferation & 14 & 0.020732215 \\
\hline GO:0048566 embryonic gut development & 3 & 0.020928455 \\
\hline GO:0042573 retinoic acid metabolic process & 3 & 0.020928455 \\
\hline GO:0009719 response to endogenous stimulus & 15 & 0.022644706 \\
\hline GO:0051174 regulation of phosphorus metabolic process & 17 & 0.02297031 \\
\hline GO:0019220 regulation of phosphate metabolic process & 17 & 0.02297031 \\
\hline GO:0009725 response to hormone stimulus & 14 & 0.02326085 \\
\hline GO:0006351 transcription, DNA-dependent & 12 & 0.023974942 \\
\hline GO:0016331 morphogenesis of embryonic epithelium & 5 & 0.024243091 \\
\hline GO:0014020 primary neural tube formation & 4 & 0.024303066 \\
\hline GO:0006775 fat-soluble vitamin metabolic process & 4 & 0.024303066 \\
\hline GO:0070633 transepithelial transport & 3 & 0.024427602 \\
\hline GO:0051726 regulation of cell cycle & 13 & 0.024755656 \\
\hline GO:0048589 developmental growth & 6 & 0.024939263 \\
\hline GO:0050769 positive regulation of neurogenesis & 5 & 0.025630326 \\
\hline GO:0032774 RNA biosynthetic process & 12 & 0.02613828 \\
\hline
\end{tabular}




\begin{tabular}{|c|c|c|}
\hline GO:0001756 somitogenesis & 4 & 0.02629014 \\
\hline GO:0045785 positive regulation of cell adhesion & 5 & 0.027064424 \\
\hline GO:0030902 hindbrain development & 5 & 0.027064424 \\
\hline GO:0007169 transmembrane receptor protein tyrosine kinase signaling pathway & 10 & 0.027491236 \\
\hline GO:0045668 negative regulation of osteoblast differentiation & 3 & 0.028146821 \\
\hline GO:0003006 reproductive developmental process & 11 & 0.028314277 \\
\hline GO:0006468 protein amino acid phosphorylation & 21 & 0.028893477 \\
\hline GO:0048545 response to steroid hormone stimulus & 9 & 0.030354731 \\
\hline GO:0007167 enzyme linked receptor protein signaling pathway & 13 & 0.030451206 \\
\hline GO:0048565 gut development & 4 & 0.03051738 \\
\hline GO:0043407 negative regulation of MAP kinase activity & 4 & 0.03051738 \\
\hline GO:0016310 phosphorylation & 24 & 0.030890604 \\
\hline GO:0051270 regulation of cell motion & 9 & 0.031228716 \\
\hline GO:0001893 maternal placenta development & 3 & 0.032076557 \\
\hline GO:0042325 regulation of phosphorylation & 16 & 0.03277587 \\
\hline $\begin{array}{l}\text { GO:0045934 negative regulation of nucleobase, nucleoside, nucleotide and } \\
\text { nucleic acid metabolic process }\end{array}$ & 17 & 0.035341015 \\
\hline GO:0007090 regulation of S phase of mitotic cell cycle & 3 & 0.036207534 \\
\hline GO:0007398 ectoderm development & 9 & 0.036435924 \\
\hline GO:0042471 ear morphogenesis & 5 & 0.036665433 \\
\hline GO:0043066 negative regulation of apoptosis & 13 & 0.038284051 \\
\hline GO:0008283 cell proliferation & 15 & 0.039144981 \\
\hline GO:0051172 negative regulation of nitrogen compound metabolic process & 17 & 0.039248832 \\
\hline GO:0001841 neural tube formation & 4 & 0.039973844 \\
\hline GO:0043069 negative regulation of programmed cell death & 13 & 0.041564695 \\
\hline GO:0006917 induction of apoptosis & 12 & 0.042136077 \\
\hline GO:0010035 response to inorganic substance & 9 & 0.042158107 \\
\hline GO:0060548 negative regulation of cell death & 13 & 0.042766221 \\
\hline GO:0012502 induction of programmed cell death & 12 & 0.043017406 \\
\hline GO:0012501 programmed cell death & 19 & 0.043551309 \\
\hline GO:0048732 gland development & 7 & 0.043969141 \\
\hline GO:0022405 hair cycle process & 4 & 0.045194271 \\
\hline GO:0001942 hair follicle development & 4 & 0.045194271 \\
\hline GO:0007368 determination of left/right symmetry & 4 & 0.045194271 \\
\hline GO:0022404 molting cycle process & 4 & 0.045194271 \\
\hline GO:0042303 molting cycle & 4 & 0.047925051 \\
\hline GO:0007162 negative regulation of cell adhesion & 4 & 0.047925051 \\
\hline GO:0045667 regulation of osteoblast differentiation & 4 & 0.047925051 \\
\hline GO:0001838 embryonic epithelial tube formation & 4 & 0.047925051 \\
\hline GO:0042633 hair cycle & 4 & 0.047925051 \\
\hline GO:0030198 extracellular matrix organization & 6 & 0.048104005 \\
\hline GO:0007166 cell surface receptor linked signal transduction & 46 & 0.048768893 \\
\hline GO:0006796 phosphate metabolic process & 27 & 0.048780288 \\
\hline GO:0006793 phosphorus metabolic process & 27 & 0.048780288 \\
\hline GO:0021587 cerebellum morphogenesis & 3 & 0.049719237 \\
\hline GO:0031128 developmental induction & 3 & 0.049719237 \\
\hline
\end{tabular}




\begin{tabular}{|c|c|c|}
\hline GO:0035115 embryonic forelimb morphogenesis & 3 & 0.049719237 \\
\hline GO:0001502 cartilage condensation & 3 & 0.049719237 \\
\hline GO:0051492 regulation of stress fiber formation & 3 & 0.049719237 \\
\hline GO:0045168 cell-cell signaling involved in cell fate specification & 3 & 0.049719237 \\
\hline GO:0043627 response to estrogen stimulus & 6 & 0.049761407 \\
\hline GO:0003007 heart morphogenesis & 5 & 0.050048384 \\
\hline GO:0007243 protein kinase cascade & 13 & 0.050540179 \\
\hline GO:0006720 isoprenoid metabolic process & 4 & 0.050735094 \\
\hline GO:0035148 tube lumen formation & 4 & 0.050735094 \\
\hline GO:0001701 in utero embryonic development & 8 & 0.051147881 \\
\hline GO:0006766 vitamin metabolic process & 5 & 0.054303387 \\
\hline GO:0044092 negative regulation of molecular function & 12 & 0.054485982 \\
\hline GO:0001825 blastocyst formation & 3 & 0.05456781 \\
\hline GO:0008299-isoprenoid biosynthetic process & 3 & 0.05456781 \\
\hline GO:0033261 regulation of S phase & 3 & 0.05456781 \\
\hline GO:0046149 pigment catabolic process & 2 & 0.055921085 \\
\hline $\begin{array}{l}\text { GO:0040037 negative regulation of fibroblast growth factor receptor signaling } \\
\text { pathway }\end{array}$ & 2 & 0.055921085 \\
\hline GO:0032536 regulation of cell projection size & 2 & 0.055921085 \\
\hline GO:0031133 regulation of axon diameter & 2 & 0.055921085 \\
\hline GO:0042167 heme catabolic process & 2 & 0.055921085 \\
\hline GO:0008219 cell death & 21 & 0.056076417 \\
\hline GO:0030111 regulation of Wnt receptor signaling pathway & 4 & 0.056589295 \\
\hline GO:0010558 negative regulation of macromolecule biosynthetic process & 17 & 0.05772314 \\
\hline GO:0016265 death & 21 & 0.058148938 \\
\hline GO:0032231 regulation of actin filament bundle formation & 3 & 0.059575223 \\
\hline GO:0009746 response to hexose stimulus & 4 & 0.05963149 \\
\hline GO:0034284 response to monosaccharide stimulus & 4 & 0.05963149 \\
\hline GO:0040007 growth & 8 & 0.060379206 \\
\hline GO:0048534 hemopoietic or lymphoid organ development & 10 & 0.060923059 \\
\hline GO:0000165 MAPKKK cascade & 8 & 0.061876376 \\
\hline GO:0008544 epidermis development & 8 & 0.061876376 \\
\hline GO:0001525 angiogenesis & 7 & 0.063230158 \\
\hline GO:0043547 positive regulation of GTPase activity & 3 & 0.064733745 \\
\hline GO:0008633 activation of pro-apoptotic gene products & 3 & 0.064733745 \\
\hline GO:0010564 regulation of cell cycle process & 6 & 0.06617626 \\
\hline GO:0006915 apoptosis & 18 & 0.067397024 \\
\hline $\begin{array}{l}\text { GO:0000122 negative regulation of transcription from RNA polymerase II } \\
\text { promoter }\end{array}$ & 10 & 0.067789829 \\
\hline GO:0007548 sex differentiation & 7 & 0.068282648 \\
\hline GO:0010817 regulation of hormone levels & 7 & 0.068282648 \\
\hline GO:0031327 negative regulation of cellular biosynthetic process & 17 & 0.068700964 \\
\hline GO:0007346 regulation of mitotic cell cycle & 7 & 0.070017571 \\
\hline GO:0048806 genitalia development & 3 & 0.070035878 \\
\hline GO:0021575 hindbrain morphogenesis & 3 & 0.070035878 \\
\hline GO:0001523 retinoid metabolic process & 3 & 0.070035878 \\
\hline GO:0006776 vitamin A metabolic process & 3 & 0.070035878 \\
\hline
\end{tabular}




\begin{tabular}{|c|c|c|}
\hline GO:0016101 diterpenoid metabolic process & 3 & 0.070035878 \\
\hline GO:0001755 neural crest cell migration & 3 & 0.070035878 \\
\hline GO:0044057 regulation of system process & 11 & 0.070928679 \\
\hline GO:0002683 negative regulation of immune system process & 5 & 0.073205344 \\
\hline GO:0010746 regulation of plasma membrane long-chain fatty acid transport & 2 & 0.073860329 \\
\hline GO:0051497 negative regulation of stress fiber formation & 2 & 0.073860329 \\
\hline GO:0060441 branching involved in lung morphogenesis & 2 & 0.073860329 \\
\hline GO:0045110 intermediate filament bundle assembly & 2 & 0.073860329 \\
\hline GO:0042904 9-cis-retinoic acid biosynthetic process & 2 & 0.073860329 \\
\hline $\begin{array}{l}\text { GO:0010748 negative regulation of plasma membrane long-chain fatty acid } \\
\text { transport }\end{array}$ & 2 & 0.073860329 \\
\hline GO:0042905 9-cis-retinoic acid metabolic process & 2 & 0.073860329 \\
\hline GO:0031558 induction of apoptosis in response to chemical stimulus & 2 & 0.073860329 \\
\hline GO:0060438 trachea development & 2 & 0.073860329 \\
\hline GO:0035238 vitamin A biosynthetic process & 2 & 0.073860329 \\
\hline GO:0033674 positive regulation of kinase activity & 9 & 0.074038316 \\
\hline GO:0045778 positive regulation of ossification & 3 & 0.075474351 \\
\hline GO:0001892 embryonic placenta development & 3 & 0.075474351 \\
\hline GO:0046777 protein amino acid autophosphorylation & 5 & 0.078390786 \\
\hline GO:0007242 intracellular signaling cascade & 32 & 0.078534519 \\
\hline GO:0001889 liver development & 4 & 0.079427237 \\
\hline GO:0009890 negative regulation of biosynthetic process & 17 & 0.080132165 \\
\hline GO:0006721 terpenoid metabolic process & 3 & 0.081042119 \\
\hline GO:0006606 protein import into nucleus & 5 & 0.081050684 \\
\hline GO:0002520 immune system development & 10 & 0.081103167 \\
\hline GO:0048609 reproductive process in a multicellular organism & 15 & 0.081411812 \\
\hline GO:0032504 multicellular organism reproduction & 15 & 0.081411812 \\
\hline GO:0030097 hemopoiesis & 9 & 0.081471717 \\
\hline GO:0042476 odontogenesis & 4 & 0.082972575 \\
\hline GO:0051170 nuclear import & 5 & 0.086502872 \\
\hline GO:0018149 peptide cross-linking & 3 & 0.08673235 \\
\hline GO:0051347 positive regulation of transferase activity & 9 & 0.087711566 \\
\hline GO:0043623 cellular protein complex assembly & 7 & 0.088754002 \\
\hline GO:0030335 positive regulation of cell migration & 5 & 0.089294329 \\
\hline GO:0042472 inner ear morphogenesis & 4 & 0.090262802 \\
\hline GO:0043062 extracellular structure organization & 7 & 0.090765091 \\
\hline $\begin{array}{l}\text { GO:0031557 induction of programmed cell death in response to chemical } \\
\text { stimulus }\end{array}$ & 2 & 0.091459996 \\
\hline GO:0045683 negative regulation of epidermis development & 2 & 0.091459996 \\
\hline GO:0006787 porphyrin catabolic process & 2 & 0.091459996 \\
\hline GO:0042362 fat-soluble vitamin biosynthetic process & 2 & 0.091459996 \\
\hline GO:0033015 tetrapyrrole catabolic process & 2 & 0.091459996 \\
\hline GO:0032232 negative regulation of actin filament bundle formation & 2 & 0.091459996 \\
\hline GO:0002695 negative regulation of leukocyte activation & 4 & 0.094005045 \\
\hline GO:0045787 positive regulation of cell cycle & 4 & 0.094005045 \\
\hline GO:0051240 positive regulation of multicellular organismal process & 9 & 0.094221975 \\
\hline GO:0010647 positive regulation of cell communication & 11 & 0.097158063 \\
\hline
\end{tabular}




\subsubsection{GO analysis of candidate genes downregulated at stage 14}

Given is the biological process, the number of genes and the P-value. Biological processes were sorted according to the P-value from lowest to highest.

Table 6.4 Summary of GO analysis of genes downregulated at stage 14

\begin{tabular}{|c|c|c|}
\hline Biological Process & Count & P-Value \\
\hline GO:0007017 microtubule-based process & 25 & $2.09 \mathrm{E}-10$ \\
\hline GO:0007018 microtubule-based movement & 16 & 6.13E-09 \\
\hline GO:0030030 cell projection organization & 25 & $3.15 \mathrm{E}-07$ \\
\hline GO:0006928 cell motion & 25 & $2.56 \mathrm{E}-05$ \\
\hline GO:0035295 tube development & 16 & 3.30E-05 \\
\hline GO:0030031 cell projection assembly & 10 & 3.76E-05 \\
\hline GO:0001539 ciliary or flagellar motility & 5 & 1.29E-04 \\
\hline GO:0060271 cilium morphogenesis & 6 & 4.43E-04 \\
\hline GO:0030324 lung development & 9 & 7.72E-04 \\
\hline GO:0030323 respiratory tube development & 9 & $9.40 \mathrm{E}-04$ \\
\hline GO:0007507 heart development & 13 & 1.22E-03 \\
\hline GO:0051674 localization of cell & 16 & $1.23 \mathrm{E}-03$ \\
\hline GO:0048870 cell motility & 16 & 1.23E-03 \\
\hline GO:0060541 respiratory system development & 9 & 1.36E-03 \\
\hline GO:0000902 cell morphogenesis & 17 & 1.99E-03 \\
\hline GO:0042384 cilium assembly & 5 & 2.12E-03 \\
\hline GO:0032989 cellular component morphogenesis & 18 & $2.43 \mathrm{E}-03$ \\
\hline GO:0048729 tissue morphogenesis & 11 & 3.17E-03 \\
\hline GO:0048858 cell projection morphogenesis & 13 & 3.64E-03 \\
\hline GO:0002009 morphogenesis of an epithelium & 8 & $3.91 \mathrm{E}-03$ \\
\hline GO:0032990 cell part morphogenesis & 13 & 5.13E-03 \\
\hline GO:0007423 sensory organ development & 12 & $6.04 \mathrm{E}-03$ \\
\hline GO:0046777 protein amino acid autophosphorylation & 7 & $6.77 \mathrm{E}-03$ \\
\hline GO:0048232 male gamete generation & 14 & 8.57E-03 \\
\hline GO:0007283 spermatogenesis & 14 & 8.57E-03 \\
\hline GO:0048286 lung alveolus development & 4 & 8.96E-03 \\
\hline GO:0007242 intracellular signaling cascade & 38 & $9.30 \mathrm{E}-03$ \\
\hline GO:0019953 sexual reproduction & 18 & 9.96E-03 \\
\hline GO:0007276 gamete generation & 16 & 1.23E-02 \\
\hline GO:0003007 heart morphogenesis & 6 & 1.48E-02 \\
\hline GO:0048609 reproductive process in a multicellular organism & 18 & 1.72E-02 \\
\hline GO:0032504 multicellular organism reproduction & 18 & 1.72E-02 \\
\hline GO:0008360 regulation of cell shape & 5 & $2.20 \mathrm{E}-02$ \\
\hline
\end{tabular}




\begin{tabular}{|c|c|c|}
\hline GO:0051350 negative regulation of lyase activity & 5 & $2.33 \mathrm{E}-02$ \\
\hline GO:0031280 negative regulation of cyclase activity & 5 & 2.33E-02 \\
\hline GO:0007194 negative regulation of adenylate cyclase activity & 5 & $2.33 \mathrm{E}-02$ \\
\hline GO:0001701 in utero embryonic development & 9 & $2.42 \mathrm{E}-02$ \\
\hline GO:0001568 blood vessel development & 11 & $2.47 \mathrm{E}-02$ \\
\hline GO:0000226 microtubule cytoskeleton organization & 8 & 2.71E-02 \\
\hline GO:0007588 excretion & 5 & 2.77E-02 \\
\hline GO:0016331 morphogenesis of embryonic epithelium & 5 & 2.77E-02 \\
\hline GO:0045893 positive regulation of transcription, DNA-dependent & 17 & 2.83E-02 \\
\hline GO:0001944 vasculature development & 11 & $2.84 \mathrm{E}-02$ \\
\hline GO:0051056 regulation of small GTPase mediated signal transduction & 11 & $2.91 \mathrm{E}-02$ \\
\hline GO:0043193 positive regulation of gene-specific transcription & 6 & $2.92 \mathrm{E}-02$ \\
\hline GO:0017157 regulation of exocytosis & 4 & 2.93E-02 \\
\hline GO:0051254 positive regulation of RNA metabolic process & 17 & 3.06E-02 \\
\hline GO:0001890 placenta development & 5 & 3.09E-02 \\
\hline GO:0022037 metencephalon development & 4 & 3.16E-02 \\
\hline GO:0051173 positive regulation of nitrogen compound metabolic process & 21 & 3.16E-02 \\
\hline GO:0031328 positive regulation of cellular biosynthetic process & 22 & 3.16E-02 \\
\hline GO:0043009 chordate embryonic development & 13 & $3.27 \mathrm{E}-02$ \\
\hline GO:0009792 embryonic development ending in birth or egg hatching & 13 & $3.44 \mathrm{E}-02$ \\
\hline GO:0035088 establishment or maintenance of apical/basal cell polarity & 3 & $3.46 \mathrm{E}-02$ \\
\hline $\begin{array}{l}\text { GO:0007169 transmembrane receptor protein tyrosine kinase signaling } \\
\text { pathway }\end{array}$ & 10 & $3.49 \mathrm{E}-02$ \\
\hline GO:0009891 positive regulation of biosynthetic process & 22 & $3.60 \mathrm{E}-02$ \\
\hline GO:0046903 secretion & 12 & $3.70 \mathrm{E}-02$ \\
\hline GO:0048545 response to steroid hormone stimulus & 9 & 3.78E-02 \\
\hline GO:0048639 positive regulation of developmental growth & 3 & $3.91 \mathrm{E}-02$ \\
\hline GO:0010628 positive regulation of gene expression & 19 & $4.08 \mathrm{E}-02$ \\
\hline GO:0007264 small GTPase mediated signal transduction & 12 & 4.09E-02 \\
\hline GO:0042475 odontogenesis of dentine-containing tooth & 4 & 4.16E-02 \\
\hline GO:0045761 regulation of adenylate cyclase activity & 6 & 4.19E-02 \\
\hline GO:0006468 protein amino acid phosphorylation & 21 & 4.25E-02 \\
\hline GO:0048598 embryonic morphogenesis & 12 & $4.31 \mathrm{E}-02$ \\
\hline GO:0050796 regulation of insulin secretion & 4 & 4.44E-02 \\
\hline GO:0007193 inhibition of adenylate cyclase activity by G-protein signaling & 4 & $4.44 \mathrm{E}-02$ \\
\hline GO:0021915 neural tube development & 5 & 4.58E-02 \\
\hline GO:0016310 phosphorylation & 24 & $4.61 \mathrm{E}-02$ \\
\hline GO:0031279 regulation of cyclase activity & 6 & $4.68 \mathrm{E}-02$ \\
\hline GO:0006796 phosphate metabolic process & 28 & 4.75E-02 \\
\hline GO:0006793 phosphorus metabolic process & 28 & 4.75E-02 \\
\hline GO:0001654 eye development & 7 & 4.75E-02 \\
\hline GO:0051046 regulation of secretion & 9 & 4.85E-02 \\
\hline GO:0016055 Wnt receptor signaling pathway & 7 & 4.90E-02 \\
\hline GO:0030817 regulation of cAMP biosynthetic process & 6 & 5.03E-02 \\
\hline
\end{tabular}




\begin{tabular}{|c|c|c|}
\hline GO:0051339 regulation of lyase activity & 6 & 5.03E-02 \\
\hline GO:0032583 regulation of gene-specific transcription & 7 & $5.05 E-02$ \\
\hline GO:0048732 gland development & 7 & $5.20 \mathrm{E}-02$ \\
\hline GO:0007010 cytoskeleton organization & 15 & 5.23E-02 \\
\hline GO:0030814 regulation of cAMP metabolic process & 6 & $5.38 \mathrm{E}-02$ \\
\hline GO:0045941 positive regulation of transcription & 18 & 5.67E-02 \\
\hline GO:0046034 ATP metabolic process & 6 & $5.76 \mathrm{E}-02$ \\
\hline GO:0043627 response to estrogen stimulus & 6 & 5.76E-02 \\
\hline GO:0046599 regulation of centriole replication & 2 & 5.83E-02 \\
\hline GO:0007498 mesoderm development & 5 & 5.91E-02 \\
\hline GO:0010557 positive regulation of macromolecule biosynthetic process & 20 & $6.20 \mathrm{E}-02$ \\
\hline GO:0002791 regulation of peptide secretion & 4 & $6.26 \mathrm{E}-02$ \\
\hline GO:0046928 regulation of neurotransmitter secretion & 3 & $6.41 \mathrm{E}-02$ \\
\hline GO:0051017 actin filament bundle formation & 3 & 6.41E-02 \\
\hline $\begin{array}{l}\text { GO:0045944 positive regulation of transcription from RNA polymerase II } \\
\text { promoter }\end{array}$ & 13 & $6.50 \mathrm{E}-02$ \\
\hline GO:0030808 regulation of nucleotide biosynthetic process & 6 & $6.75 \mathrm{E}-02$ \\
\hline GO:0030802 regulation of cyclic nucleotide biosynthetic process & 6 & $6.75 \mathrm{E}-02$ \\
\hline GO:0001947 heart looping & 3 & 6.97E-02 \\
\hline $\begin{array}{l}\text { GO:0045935 positive regulation of nucleobase, nucleoside, nucleotide and } \\
\text { nucleic acid metabolic process }\end{array}$ & 19 & 7.11E-02 \\
\hline GO:0048839 inner ear development & 5 & 7.16E-02 \\
\hline GO:0019216 regulation of lipid metabolic process & 6 & $7.18 \mathrm{E}-02$ \\
\hline GO:0030799 regulation of cyclic nucleotide metabolic process & 6 & 7.39E-02 \\
\hline GO:0007044 cell-substrate junction assembly & 3 & 7.53E-02 \\
\hline GO:0002088 lens development in camera-type eye & 3 & 7.53E-02 \\
\hline GO:0006140 regulation of nucleotide metabolic process & 6 & 8.07E-02 \\
\hline GO:0009205 purine ribonucleoside triphosphate metabolic process & 6 & 8.30E-02 \\
\hline GO:0060429 epithelium development & 9 & 8.33E-02 \\
\hline GO:0009199 ribonucleoside triphosphate metabolic process & 6 & 8.53E-02 \\
\hline GO:0006357 regulation of transcription from RNA polymerase II promoter & 21 & 8.55E-02 \\
\hline GO:0010604 positive regulation of macromolecule metabolic process & 24 & 8.55E-02 \\
\hline GO:0042733 embryonic digit morphogenesis & 3 & 8.71E-02 \\
\hline GO:0042476 odontogenesis & 4 & $9.14 \mathrm{E}-02$ \\
\hline GO:0042692 muscle cell differentiation & 6 & $9.26 \mathrm{E}-02$ \\
\hline GO:0051588 regulation of neurotransmitter transport & 3 & 9.31E-02 \\
\hline GO:0009144 purine nucleoside triphosphate metabolic process & 6 & 9.51E-02 \\
\hline GO:0060411 heart septum morphogenesis & 2 & $9.52 \mathrm{E}-02$ \\
\hline GO:0030182 neuron differentiation & 14 & $9.64 \mathrm{E}-02$ \\
\hline GO:0009612 response to mechanical stimulus & 4 & $9.93 \mathrm{E}-02$ \\
\hline
\end{tabular}




\subsubsection{GO analysis of candidate genes upregulated at stage 27}

Given is the biological process, the number of genes and the P-value. Biological processes were sorted according to the P-value from lowest to highest.

Table 6.5 Summary of GO analysis of genes upregulated at stage 27

\begin{tabular}{|c|c|c|}
\hline Biological Process & Count & P-value \\
\hline GO:0030182 neuron differentiation & 41 & $1.16 \mathrm{E}-16$ \\
\hline GO:0031175 neuron projection development & 29 & 8.25E-14 \\
\hline GO:0048666 neuron development & 32 & $4.71 \mathrm{E}-13$ \\
\hline GO:0048667 cell morphogenesis involved in neuron differentiation & 25 & 1.88E-12 \\
\hline GO:0048812 neuron projection morphogenesis & 25 & $2.84 \mathrm{E}-12$ \\
\hline GO:0000904 cell morphogenesis involved in differentiation & 26 & $8.19 \mathrm{E}-12$ \\
\hline GO:0007409 axonogenesis & 23 & $1.90 \mathrm{E}-11$ \\
\hline GO:0030030 cell projection organization & 31 & $2.15 \mathrm{E}-11$ \\
\hline GO:0048858 cell projection morphogenesis & 25 & 5.67E-11 \\
\hline GO:0032990 cell part morphogenesis & 25 & $1.42 \mathrm{E}-10$ \\
\hline GO:0000902 cell morphogenesis & 29 & $2.41 \mathrm{E}-10$ \\
\hline GO:0032989 cellular component morphogenesis & 30 & $6.46 \mathrm{E}-10$ \\
\hline GO:0007389 pattern specification process & 24 & 1.85E-09 \\
\hline GO:0007155 cell adhesion & 39 & $6.09 \mathrm{E}-09$ \\
\hline GO:0022610 biological adhesion & 39 & $6.21 \mathrm{E}-09$ \\
\hline GO:0048706 embryonic skeletal system development & 13 & 2.39E-08 \\
\hline GO:0043009 chordate embryonic development & 25 & $2.43 \mathrm{E}-08$ \\
\hline GO:0009792 embryonic development ending in birth or egg hatching & 25 & 2.89E-08 \\
\hline GO:0045664 regulation of neuron differentiation & 16 & 3.87E-08 \\
\hline GO:0009952 anterior/posterior pattern formation & 16 & 7.73E-08 \\
\hline GO:0010975 regulation of neuron projection development & 12 & 8.50E-08 \\
\hline GO:0050767 regulation of neurogenesis & 17 & 1.26E-07 \\
\hline GO:0007411 axon guidance & 14 & $1.31 \mathrm{E}-07$ \\
\hline GO:0051960 regulation of nervous system development & 18 & 1.74E-07 \\
\hline GO:0001501 skeletal system development & 23 & 2.27E-07 \\
\hline GO:0003002 regionalization & 18 & $2.52 \mathrm{E}-07$ \\
\hline GO:0007268 synaptic transmission & 22 & 3.05E-07 \\
\hline GO:0006928 cell motion & 28 & 4.70E-07 \\
\hline GO:0006836 neurotransmitter transport & 12 & 5.09E-07 \\
\hline GO:0031344 regulation of cell projection organization & 12 & 1.04E-06 \\
\hline GO:0019226 transmission of nerve impulse & 23 & $1.11 \mathrm{E}-06$ \\
\hline GO:0050770 regulation of axonogenesis & 10 & $1.25 \mathrm{E}-06$ \\
\hline GO:0007267 cell-cell signaling & 31 & 1.57E-06 \\
\hline GO:0048598 embryonic morphogenesis & 21 & $2.02 \mathrm{E}-06$ \\
\hline GO:0060284 regulation of cell development & 17 & $2.18 \mathrm{E}-06$ \\
\hline
\end{tabular}




\begin{tabular}{|c|c|c|}
\hline GO:0016337 cell-cell adhesion & 19 & $6.52 \mathrm{E}-06$ \\
\hline GO:0048066 pigmentation during development & 7 & 7.93E-06 \\
\hline GO:0048705 skeletal system morphogenesis & 12 & 1.01E-05 \\
\hline GO:0006813 potassium ion transport & 14 & 1.29E-05 \\
\hline GO:0010769 regulation of cell morphogenesis involved in differentiation & 10 & 1.61E-05 \\
\hline GO:0051969 regulation of transmission of nerve impulse & 13 & 2.65E-05 \\
\hline GO:0048568 embryonic organ development & 14 & 2.79E-05 \\
\hline GO:0030001 metal ion transport & 24 & 3.29E-05 \\
\hline GO:0031644 regulation of neurological system process & 13 & 3.95E-05 \\
\hline GO:0022604 regulation of cell morphogenesis & 12 & 4.44E-05 \\
\hline GO:0043062 extracellular structure organization & 13 & 7.32E-05 \\
\hline GO:0044057 regulation of system process & 18 & 1.01E-04 \\
\hline GO:0048704 embryonic skeletal system morphogenesis & 8 & 1.04E-04 \\
\hline GO:0007610 behavior & 23 & 1.07E-04 \\
\hline GO:0015672 monovalent inorganic cation transport & 18 & 1.43E-04 \\
\hline GO:0030814 regulation of cAMP metabolic process & 10 & 1.62E-04 \\
\hline GO:0048562 embryonic organ morphogenesis & 11 & 2.46E-04 \\
\hline GO:0001505 regulation of neurotransmitter levels & 8 & 2.91E-04 \\
\hline GO:0050804 regulation of synaptic transmission & 11 & 2.94E-04 \\
\hline GO:0030799 regulation of cyclic nucleotide metabolic process & 10 & $3.26 \mathrm{E}-04$ \\
\hline GO:0042438 melanin biosynthetic process & 4 & 3.66E-04 \\
\hline GO:0006140 regulation of nucleotide metabolic process & 10 & 3.97E-04 \\
\hline GO:0006812 cation transport & 24 & 4.26E-04 \\
\hline GO:0016358 dendrite development & 6 & 5.11E-04 \\
\hline GO:0051046 regulation of secretion & 13 & $5.39 \mathrm{E}-04$ \\
\hline GO:0006582 melanin metabolic process & 4 & $5.41 \mathrm{E}-04$ \\
\hline GO:0032940 secretion by cell & 13 & $6.70 \mathrm{E}-04$ \\
\hline GO:0030817 regulation of cAMP biosynthetic process & 9 & 7.24E-04 \\
\hline GO:0007626 locomotory behavior & 15 & 8.59E-04 \\
\hline GO:0043473 pigmentation & 7 & $9.14 \mathrm{E}-04$ \\
\hline GO:0016477 cell migration & 15 & 9.23E-04 \\
\hline GO:0003001 generation of a signal involved in cell-cell signaling & 8 & 0.001231481 \\
\hline GO:0030802 regulation of cyclic nucleotide biosynthetic process & 9 & 0.001265542 \\
\hline GO:0030808 regulation of nucleotide biosynthetic process & 9 & 0.001265542 \\
\hline GO:0006811 ion transport & 28 & 0.001768227 \\
\hline GO:0048870 cell motility & 15 & 0.0025172 \\
\hline GO:0051674 localization of cell & 15 & 0.0025172 \\
\hline GO:0001568 blood vessel development & 13 & 0.002811991 \\
\hline GO:0035108- limb morphogenesis & 8 & 0.002961616 \\
\hline GO:0035107 appendage morphogenesis & 8 & 0.002961616 \\
\hline GO:0060341 regulation of cellular localization & 13 & 0.003114642 \\
\hline GO:0035239 tube morphogenesis & 9 & 0.003134576 \\
\hline GO:0021700 developmental maturation & 8 & 0.00331301 \\
\hline GO:0001944 vasculature development & 13 & 0.003419115 \\
\hline GO:0048489 synaptic vesicle transport & 5 & 0.003519358 \\
\hline GO:0035295 tube development & 12 & 0.003569016 \\
\hline
\end{tabular}




\begin{tabular}{|c|c|c|}
\hline GO:0048736 appendage development & 8 & 0.003695362 \\
\hline GO:0060173 limb development & 8 & 0.003695362 \\
\hline GO:0007156 homophilic cell adhesion & 9 & 0.003789648 \\
\hline GO:0030198 extracellular matrix organization & 8 & 0.00389868 \\
\hline GO:0017157 regulation of exocytosis & 5 & 0.003929277 \\
\hline GO:0007269 neurotransmitter secretion & 5 & 0.003929277 \\
\hline GO:0046903 secretion & 14 & 0.005404261 \\
\hline GO:0032535 regulation of cellular component size & 13 & 0.006246604 \\
\hline GO:0055085 transmembrane transport & 21 & 0.006911794 \\
\hline GO:0046928 regulation of neurotransmitter secretion & 4 & 0.007224663 \\
\hline GO:0030815 negative regulation of cAMP metabolic process & 3 & 0.007250275 \\
\hline GO:0034109 homotypic cell-cell adhesion & 3 & 0.007250275 \\
\hline GO:0060425 lung morphogenesis & 3 & 0.007250275 \\
\hline GO:0060216 definitive hemopoiesis & 3 & 0.007250275 \\
\hline GO:0030818 negative regulation of cAMP biosynthetic process & 3 & 0.007250275 \\
\hline GO:0048754 branching morphogenesis of a tube & 6 & 0.008159968 \\
\hline GO:0007157 heterophilic cell adhesion & 4 & 0.008248185 \\
\hline GO:0045596 negative regulation of cell differentiation & 11 & 0.009099984 \\
\hline GO:0060562 epithelial tube morphogenesis & 6 & 0.009251241 \\
\hline GO:0030800 negative regulation of cyclic nucleotide metabolic process & 3 & 0.009545066 \\
\hline GO:0030803 negative regulation of cyclic nucleotide biosynthetic process & 3 & 0.009545066 \\
\hline GO:0030809 negative regulation of nucleotide biosynthetic process & 3 & 0.009545066 \\
\hline GO:0045761 regulation of adenylate cyclase activity & 7 & 0.010452748 \\
\hline GO:0007605 sensory perception of sound & 7 & 0.010967908 \\
\hline GO:0031279 regulation of cyclase activity & 7 & 0.012051707 \\
\hline GO:0051588 regulation of neurotransmitter transport & 4 & 0.013163109 \\
\hline GO:0002009 morphogenesis of an epithelium & 7 & 0.013208821 \\
\hline GO:0051339 regulation of lyase activity & 7 & 0.013208821 \\
\hline GO:0001763 morphogenesis of a branching structure & 6 & 0.013856403 \\
\hline GO:0051216 cartilage development & 6 & 0.013856403 \\
\hline GO:0050954 sensory perception of mechanical stimulus & 7 & 0.014441604 \\
\hline GO:0031103 axon regeneration & 3 & 0.014956548 \\
\hline GO:0007413 axonal fasciculation & 3 & 0.014956548 \\
\hline GO:0045980 negative regulation of nucleotide metabolic process & 3 & 0.014956548 \\
\hline GO:0050877 neurological system process & 35 & 0.015730745 \\
\hline GO:0060485 mesenchyme development & 5 & 0.017544866 \\
\hline GO:0019748 secondary metabolic process & 6 & 0.017956203 \\
\hline GO:0008344 adult locomotory behavior & 5 & 0.019898081 \\
\hline GO:0001701 in utero embryonic development & 9 & 0.020509356 \\
\hline GO:0031102 neuron projection regeneration & 3 & 0.021389283 \\
\hline GO:0000768 syncytium formation by plasma membrane fusion & 3 & 0.021389283 \\
\hline GO:0007416 synaptogenesis & 4 & 0.023087282 \\
\hline GO:0048729 -tissue morphogenesis & 9 & 0.023132422 \\
\hline GO:0006887 exocytosis & 7 & 0.02355024 \\
\hline GO:0030534 adult behavior & 6 & 0.024933099 \\
\hline GO:0014032 neural crest cell development & 4 & 0.025044074 \\
\hline
\end{tabular}




\begin{tabular}{|c|c|c|}
\hline GO:0014033 neural crest cell differentiation & 4 & 0.025044074 \\
\hline GO:0000165 MAPKKK cascade & 9 & 0.02591496 \\
\hline GO:0030326 embryonic limb morphogenesis & 6 & 0.026053265 \\
\hline GO:0035113 embryonic appendage morphogenesis & 6 & 0.026053265 \\
\hline GO:0006949 syncytium formation & 3 & 0.028758271 \\
\hline GO:0030318 melanocyte differentiation & 3 & 0.028758271 \\
\hline GO:0048488 synaptic vesicle endocytosis & 3 & 0.028758271 \\
\hline GO:0050808 synapse organization & 5 & 0.029616122 \\
\hline GO:0031290 retinal ganglion cell axon guidance & 3 & 0.032768659 \\
\hline GO:0050931 pigment cell differentiation & 3 & 0.032768659 \\
\hline GO:0001667 ameboidal cell migration & 4 & 0.03373438 \\
\hline GO:0001764 neuron migration & 5 & 0.034504837 \\
\hline GO:0048167 regulation of synaptic plasticity & 5 & 0.034504837 \\
\hline GO:0060627 regulation of vesicle-mediated transport & 6 & 0.037588508 \\
\hline GO:0048791 calcium ion-dependent exocytosis of neurotransmitter & 2 & 0.038070802 \\
\hline GO:0006583 melanin biosynthetic process from tyrosine & 2 & 0.038070802 \\
\hline GO:0046148 pigment biosynthetic process & 4 & 0.038592163 \\
\hline GO:0033555 multicellular organismal response to stress & 4 & 0.038592163 \\
\hline GO:0007229 integrin-mediated signaling pathway & 5 & 0.045608009 \\
\hline GO:0008361 regulation of cell size & 9 & 0.045875901 \\
\hline GO:0006029 proteoglycan metabolic process & 4 & 0.049308912 \\
\hline GO:0021545 cranial nerve development & 3 & 0.050763034 \\
\hline GO:0016192 vesicle-mediated transport & 18 & 0.052921235 \\
\hline GO:0042440 pigment metabolic process & 4 & 0.05515485 \\
\hline GO:0048678 response to axon injury & 3 & 0.05570552 \\
\hline GO:0030516 regulation of axon extension & 3 & 0.05570552 \\
\hline GO:0008038 neuron recognition & 3 & 0.060808768 \\
\hline GO:0040007 growth & 8 & 0.063432166 \\
\hline GO:0007628 adult walking behavior & 3 & 0.066064826 \\
\hline GO:0045103 intermediate filament-based process & 3 & 0.066064826 \\
\hline GO:0035136 forelimb morphogenesis & 3 & 0.066064826 \\
\hline GO:0007167 enzyme linked receptor protein signaling pathway & 12 & 0.06630625 \\
\hline GO:0007018 microtubule-based movement & 6 & 0.066795171 \\
\hline GO:0010817 regulation of hormone levels & 7 & 0.071348392 \\
\hline GO:0009954 proximal/distal pattern formation & 3 & 0.071465987 \\
\hline GO:0001755 neural crest cell migration & 3 & 0.071465987 \\
\hline GO:0014031 mesenchymal cell development & 4 & 0.074545726 \\
\hline GO:0048762 mesenchymal cell differentiation & 4 & 0.074545726 \\
\hline GO:0060441 branching involved in lung morphogenesis & 2 & 0.07469758 \\
\hline GO:0060438 trachea development & 2 & 0.07469758 \\
\hline GO:0006904 vesicle docking during exocytosis & 3 & 0.077004782 \\
\hline GO:0016050 vesicle organization & 4 & 0.078035575 \\
\hline GO:0001558 regulation of cell growth & 8 & 0.080849044 \\
\hline GO:0050771 negative regulation of axonogenesis & 3 & 0.082673971 \\
\hline GO:0050772 positive regulation of axonogenesis & 3 & 0.082673971 \\
\hline GO:0051970 negative regulation of transmission of $n$ & 3 & 0.082673971 \\
\hline
\end{tabular}




\begin{tabular}{|l|r|r|}
\hline GO:0021675 nerve development & 3 & 0.082673971 \\
\hline GO:0009611 response to wounding & 16 & 0.083449246 \\
\hline GO:0042476 odontogenesis & 4 & 0.085225078 \\
\hline GO:0048589 developmental growth & 5 & 0.086531155 \\
\hline GO:0006355 regulation of transcription, DNA-dependent & 43 & 0.086826183 \\
\hline GO:0007242 intracellular signaling cascade & 32 & 0.088261636 \\
\hline GO:0018149 peptide cross-linking & 3 & 0.088466543 \\
\hline GO:0048278 vesicle docking & 3 & 0.088466543 \\
\hline GO:0008037 cell recognition & 4 & 0.088922057 \\
\hline GO:0031280 negative regulation of cyclase activity & 4 & 0.088922057 \\
\hline GO:0051350 negative regulation of lyase activity & 4 & 0.088922057 \\
\hline GO:0007194 negative regulation of adenylate cyclase activity & 4 & 0.088922057 \\
\hline GO:0007160 cell-matrix adhesion & 5 & 0.09222525 \\
\hline GO:0032229 negative regulation of synaptic transmission, GABAergic & 2 & 0.092486595 \\
\hline GO:0016188 synaptic vesicle maturation & 2 & 0.092486595 \\
\hline
\end{tabular}

\subsubsection{GO analysis of candidate genes downregulated at stage 27}

Given is the biological process, the number of genes and the P-value. Biological processes were sorted according to the P-value from lowest to highest.

Table 6.6 Summary of GO analysis of genes downregulated at stage 27

\begin{tabular}{|l|r|r|}
\hline Biological Process & Count & \multicolumn{1}{l|}{ P-Value } \\
\hline GO:0042398 cellular amino acid derivative biosynthetic process & 3 & 0.004423821 \\
\hline GO:0009309 amine biosynthetic process & 3 & 0.009715815 \\
\hline GO:0042423 catecholamine biosynthetic process & 2 & 0.020148791 \\
\hline GO:0006575 cellular amino acid derivative metabolic process & 3 & 0.037332173 \\
\hline GO:0007595 lactation & 2 & 0.050523221 \\
\hline GO:0042401 biogenic amine biosynthetic process & 2 & 0.054036675 \\
\hline GO:0007610 behavior & 4 & 0.054132144 \\
\hline GO:0006584 catecholamine metabolic process & 2 & 0.061026178 \\
\hline GO:0009712 catechol metabolic process & 2 & 0.061026178 \\
\hline GO:0034311 diol metabolic process & 2 & 0.061026178 \\
\hline GO:0018958 phenol metabolic process & 2 & 0.062765791 \\
\hline GO:0051591 response to cAMP & 2 & 0.074856742 \\
\hline GO:0032102 negative regulation of response to external stimulus & 2 & 0.088491623 \\
\hline GO:0007626 locomotory behavior & 3 & 0.090424381 \\
\hline
\end{tabular}

